\title{
Immune regulation by mimethyl fumarate (DMF) in relapsing-remitting multiple sclerosis patients
}

Citation for published version (APA):

Montes Diaz, G. (2020). Immune regulation by mimethyl fumarate (DMF) in relapsing-remitting multiple sclerosis patients. [Doctoral Thesis, Maastricht University, tUL-Universiteit Hasselt (UHasselt)]. UHasselt / Maastricht University. https://doi.org/10.26481/dis.20201015gmd

Document status and date:

Published: 01/01/2020

DOI:

10.26481/dis.20201015gmd

Document Version:

Publisher's PDF, also known as Version of record

\section{Please check the document version of this publication:}

- A submitted manuscript is the version of the article upon submission and before peer-review. There can be important differences between the submitted version and the official published version of record.

People interested in the research are advised to contact the author for the final version of the publication, or visit the DOI to the publisher's website.

- The final author version and the galley proof are versions of the publication after peer review.

- The final published version features the final layout of the paper including the volume, issue and page numbers.

Link to publication

\footnotetext{
General rights rights.

- You may freely distribute the URL identifying the publication in the public portal. please follow below link for the End User Agreement:

www.umlib.nl/taverne-license

Take down policy

If you believe that this document breaches copyright please contact us at:

repository@maastrichtuniversity.nl

providing details and we will investigate your claim.
}

Copyright and moral rights for the publications made accessible in the public portal are retained by the authors and/or other copyright owners and it is a condition of accessing publications that users recognise and abide by the legal requirements associated with these

- Users may download and print one copy of any publication from the public portal for the purpose of private study or research.

- You may not further distribute the material or use it for any profit-making activity or commercial gain

If the publication is distributed under the terms of Article $25 \mathrm{fa}$ of the Dutch Copyright Act, indicated by the "Taverne" license above, 
2020 | Faculty of Medicine and Life Sciences

Maastricht University

Doctoral dissertation submitted to obtain the degrees of

- Doctor of Biomedical Sciences | tUL

- Doctor | Maastricht University

Gwendoline Montes Diaz

\section{DOCTORAL DISSERTATION}

Immune regulation by dimethyl fumarate (DMF) in relapsingremitting multiple sclerosis patients.

\section{BIOMED}

BIOMEDICAL

RESEARCH INSTITUTE

\section{$\rightarrow$ UHASSELT}

Promoters: $\quad$ Prof. Dr Veerle Somers | UHasselt

$D / 2020 / 2451 / 26$

Prof. Dr Raymond Hupperts | Universiteit Maastricht

Co-promoter: Dr Judith Fraussen | UHasselt 


\title{
Immune regulation by dimethyl fumarate (DMF) in relapsing-remitting multiple sclerosis patients
}

\author{
PROEFSCHRIFT
}

ter verkrijging van de graad van doctor aan de Universiteit Maastricht, op gezag van de Rector Magnificus, Prof. dr. Rianne M. Letschert,

en de graad van doctor in de Biomedische Wetenschappen aan de

Transnationale Universiteit Limburg/Universiteit Hasselt, op gezag van de Rector

Prof. dr. Luc De Schepper,

volgens het besluit van het College van Decanen,

in het openbaar te verdedigen

op donderdag 15 oktober 2020 om 10.00 uur in Maastricht

door

Gwendoline Montes Diaz 


\section{Promotores}

Prof. dr. Veerle Somers, Hasselt University, Belgium,

Prof. dr. Raymond Hupperts, Maastricht University, The Netherlands

\section{Copromotores}

dr. Judith Fraussen, Hasselt University, Belgium

\section{Assessment Committee:}

Prof. dr. Marc De Baets, Maastricht University, The Netherlands, chairman

Prof. dr. Niels Hellings, Hasselt University, Belgium

Prof. dr. Peter Feys, Hasselt University, Belgium

Prof. dr. Bert Op 't Eijnde, Hasselt University, Belgium

dr. Jan Damoiseaux, Maastricht University, The Netherlands

dr. Luisa Maria Villar, Hospital Universitario Ramón y Cajal, Spain

Prof. dr. Sandra Amor, Amsterdam UMC, The Netherlands 
"Success is not the key to happiness. Happiness is the key to success. Ifyou love what you are doing, you will be successful." - Albert Schweitzer 



\section{Table of Contents}

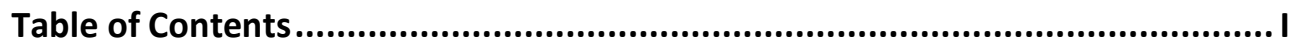

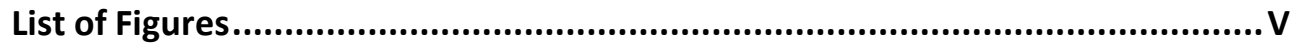

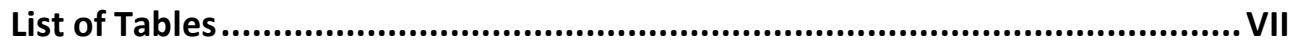

List of Abbreviations ..................................................................................... VII

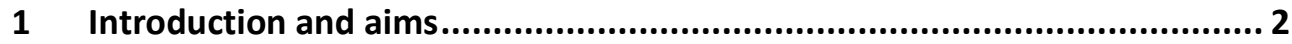

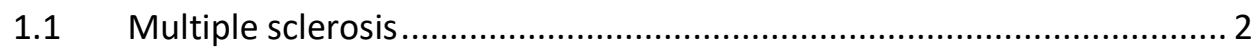

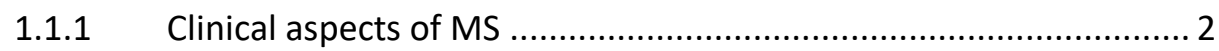

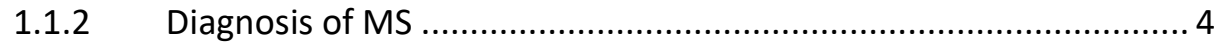

1.1.3 Immunopathogenesis of MS ...................................................... 4

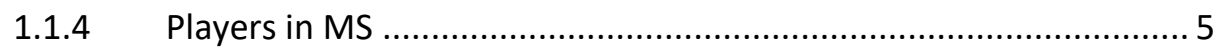

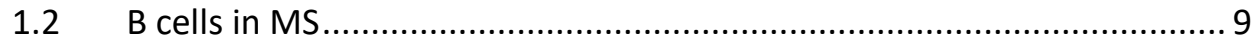

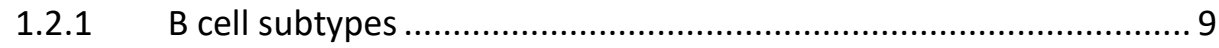

1.2.2 Pathological functions of $B$ cells in MS..................................... 13

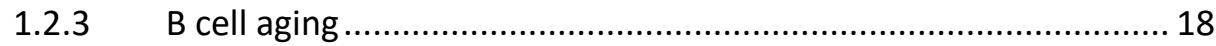

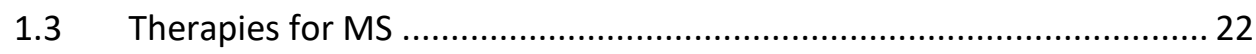

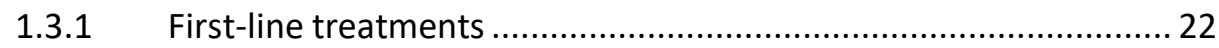

1.3.2 Second- and third-line treatments............................................. 23

1.3.3 Off-label treatments for MS......................................................... 25

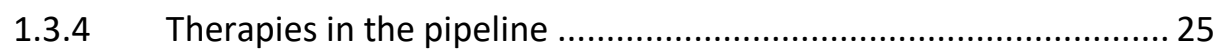

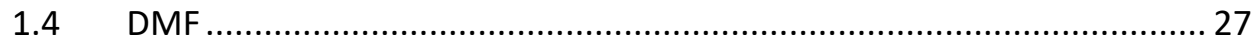

1.4.1 History of DMF as a therapeutic agent ..................................... 27

1.4.2 Pharmacokinetics of DMF ........................................................ 28

1.4.3 Molecular mechanism of action of DMF ..................................... 28

1.4.4 Efficacy of DMF in animal models for MS ................................... 34

1.4.5 Clinical trials of DMF treatment in RRMS patients........................ 34

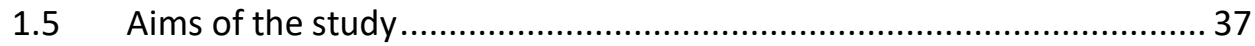

2 Dimethyl fumarate induces a persistent change in the composition of the innate and adaptive immune system in multiple sclerosis patients ................. 42 


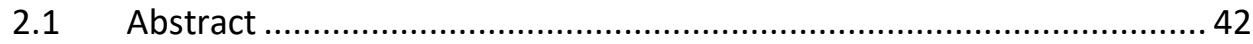

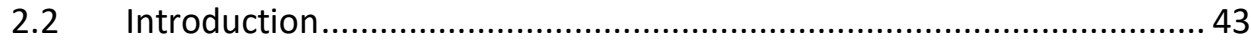

2.3 Materials and methods....................................................................... 45

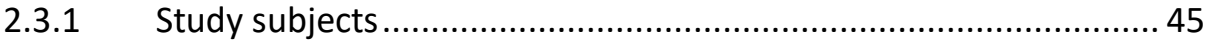

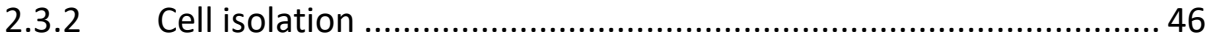

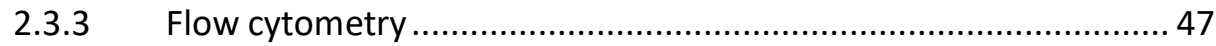

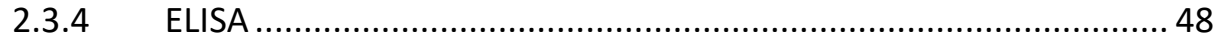

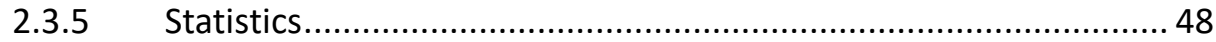

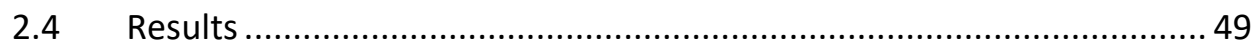

2.4.1 Clinical characteristics............................................................... 49

2.4.2 Reduced absolute numbers of lymphocytes, total T cells and B cells

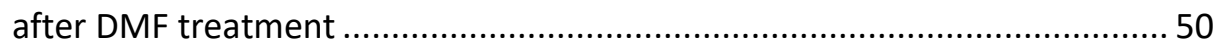

2.4.3 Percentages of innate immune cells increase after $12 \mathrm{~m}$ of DMF treatment

2.4.4 Percentages of pro-inflammatory $\mathrm{T}$ and $\mathrm{B}$ cells decrease after $12 \mathrm{~m}$ of DMF treatment, while anti-inflammatory $T$ and $B$ cells increase .............. 53

2.4.5 DMF exerts its full effect on immune cells after $6 \mathrm{~m}$ of treatment in

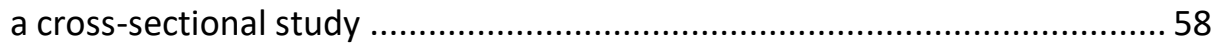

2.4.6 No effect of DMF treatment on functional markers and survival

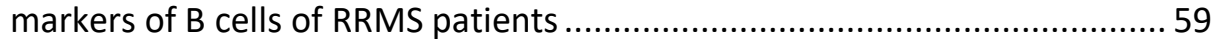

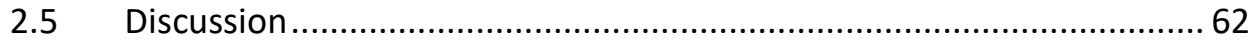

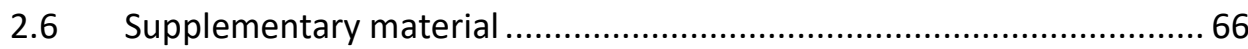

3 DMF directly affects the expression of survival and functional markers of $B$ cells 72

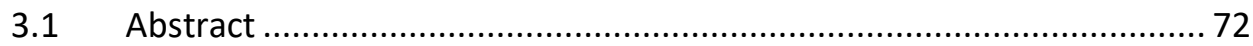

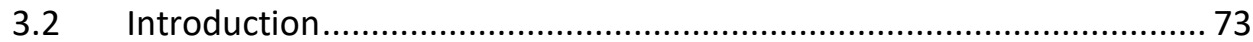

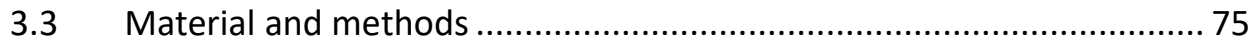

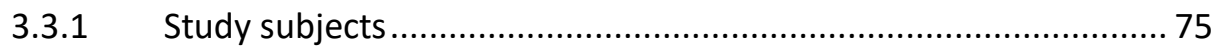

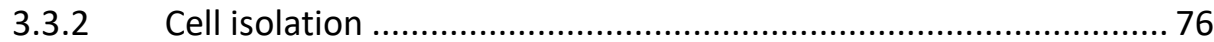

3.3.3 In vitro B cell cultures ........................................................... 76 


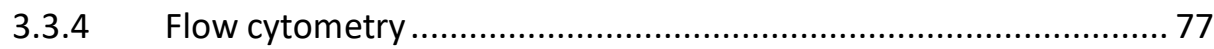

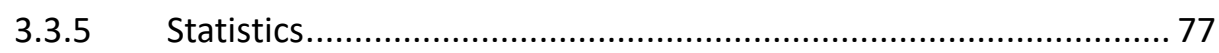

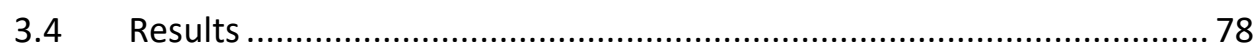

3.4.1 DMF but not MMF induces B cell apoptosis in a direct and

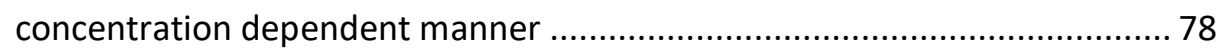

3.4.2 DMF directly reduces BAFFR expression on B cells ....................... 81

3.4.3 DMF directly reduces the expression of antigen presentation molecules and costimulatory molecule CD40 on B cells.............................. 82

3.4.4 DMF tends to directly affect frequencies of pro- and anti-

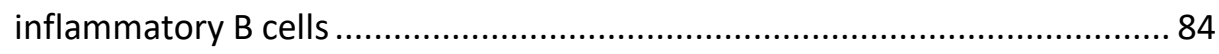

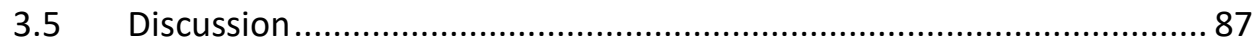

4 Phenotypical and functional characterization of IgD-CD27- double negative (DN) B cells in multiple sclerosis patients ................................................... 92

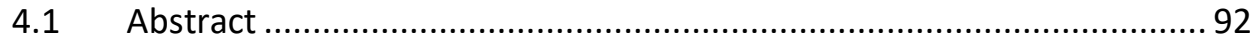

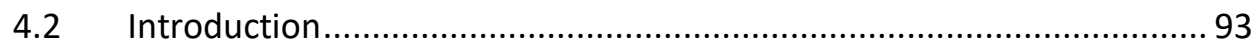

4.3 Material and methods ................................................................... 96

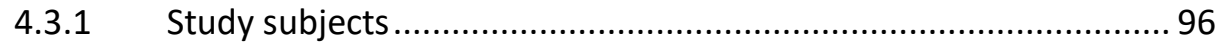

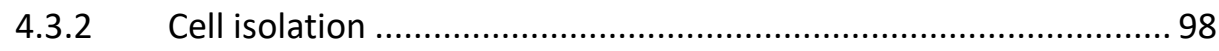

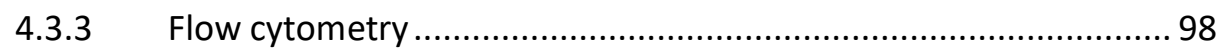

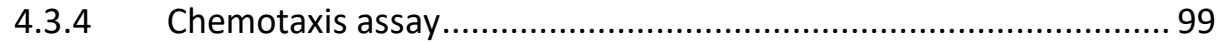

4.3.5 Proliferation and activation assay............................................. 100

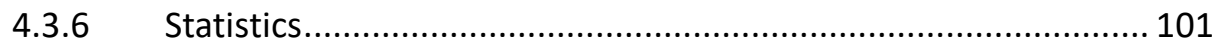

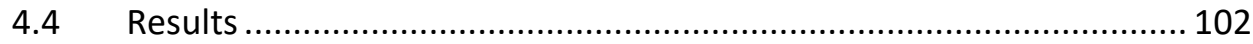

4.4.1 Total B cells and DN B cells are increased in the peripheral blood of MS patients 102

4.4.2 DN B cells resemble CSM B cells in the expression of developmental

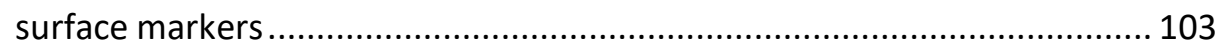

4.4.3 CD21 ${ }^{\text {low }} B$ cells show the highest expression of T-bet, while a proportion of DN B cells express T-bet..................................................... 106

4.4.4 The expression of adhesion molecules and chemokine receptor CXCR3 of DN B cells is between that of naive and CSM B cells................... 107 
4.4.5 DN B cells migrate towards CXCL13 and CXCL10 in vitro 110

4.4.6 Proliferation and activation profile of DN B cells compared to naive, NCSM and CSM B cells 112

4.5 Discussion 114

5 Summary and general discussion..................................................... 120

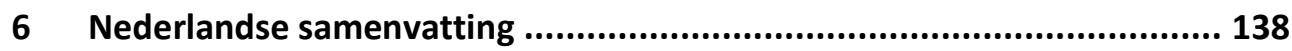

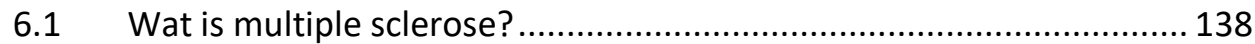

6.2 Welke immuuncellen spelen een rol bij MS? ...................................... 139

6.3 Welke behandelingen zijn er voor MS? ............................................ 140

6.4 Wat is het doel van de studie? ......................................................... 140

6.5 Wat zijn de resultaten van de studie? .............................................. 141

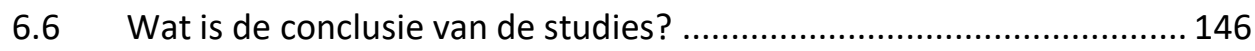

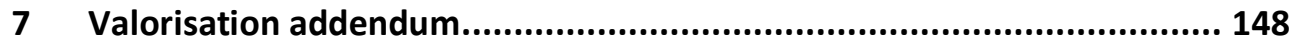

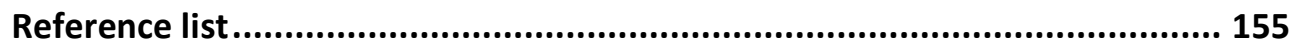

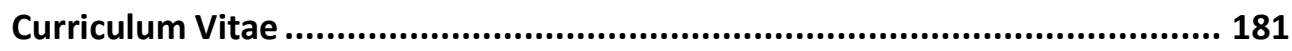

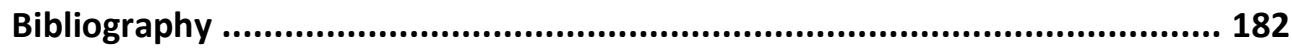

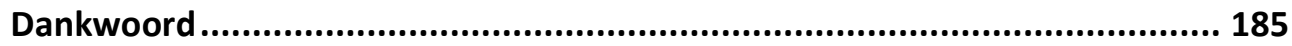




\section{List of Figures}

Figure 1.1

Figure 1.2

Figure 1.3

Figure 1.4

Figure 2.1

Figure 2.2

Figure 2.3

Figure 2.4

Figure $\mathbf{2 . 5}$

Figure 2.6

Supplementary

Figure 2.1

Supplementary

Figure 2.2

Figure 3.1

Figure $\mathbf{3 . 2}$
Pathogenesis of MS.

$B$ cell subtypes in MS.

$B$ cell functions in MS.

Molecular pathways of DMF treatment.

DMF treatment increased percentages of monocytes and NK cells.

DMF treatment reduced (effector) memory $\mathrm{T}$ cell 54 percentages after $12 \mathrm{~m}$ of treatment in MS patients.

Reduction in the percentages of T cells expressing pro-inflammatory cytokines after $12 \mathrm{~m}$ of DMF treatment.

DMF treatment reduced percentages of memory 57 and DN B cell subtypes after $12 \mathrm{~m}$ of treatment in MS patients.

DMF treatment is fully effective on immune cells after $6 \mathrm{~m}$ of treatment.

DMF treatment does not influence the expression of 60 antigen-presentation molecules, costimulatory molecules and survival markers on B cells.

DMF treatment induced redistribution of innate and 66 adaptive immune cell subtypes after $12 \mathrm{~m}$ of treatment in RRMS patients, while almost no significant changes were detected at $3 \mathrm{~m}$ of DMF treatment.

DMF treatment reduced frequency of $\mathrm{T}$ cells 68 expressing pro-inflammatory cytokines after $12 \mathrm{~m}$, but not after $3 \mathrm{~m}$ of treatment.

DMF induces apoptosis of B cells in a concentration 79 dependent manner.

MMF has no effect on apoptosis of B cells. 
Figure 3.3 DMF downregulates the expression of survival marker BAFFR on B cells.

Figure 3.4

DMF downregulates the expression of antigen

83

presentation molecules and costimulatory molecule CD40 on B cells.

Figure $\mathbf{3 . 5}$

A trend towards an increase in Breg is induced by

DMF while MMF tends to decrease TNF- $a+B$ cells in a direct manner.

Figure 4.1

Figure 4.2

Figure 4.3

Figure 4.4

Figure 4.5

Figure 4.6

Figure 5.1
Total B cells and DN B cells are increased in MS patients compared to $\mathrm{HC}$.

DN B cells of HC and MS patients resemble CSM B 105 cells in the expression of developmental surface markers, however in an earlier maturation state. CD21low B cells show the highest expression of T107 bet in $\mathrm{HC}$ and MS patients.

DN B cells of $\mathrm{HC}$ and MS patients show an 109 intermediate expression of adhesion molecules and chemokine receptor CXCR3 between naive and CSM B cells.

DN B cells of MS patients show an in vitro migration 111 capacity towards CXCL10 and CXCL13 that is comparable to that of CSM B cells.

Activation potential of naive, DN, NCSM and CSM B 113 cells after TLR9 stimulation.

Effects of DMF treatment on immune cells of MS patients. 
VII

\section{List of Tables}

Table 2.1 Characteristics of study subjects 46

Table 2.2 Clinical tests and absolute numbers of immune cells 51 after DMF treatment

Table 3.1 Characteristics of study subjects 75

$\begin{array}{lll}\text { Table 4.1 } & \text { Characteristics of study subjects } & 97\end{array}$ 


\section{List of Abbreviations}

ABCB1 ATP-binding-cassette-B1

AKR1B10 Aldo-keto reductase family 1 member B10

ALCAM Activated leukocyte cell adhesion molecule

AMP Adenosine monophosphate

ANO2 Anoctamin 2

APC Antigen presenting cells

APRIL Proliferation-inducing ligand

ARE Antioxidant response element

BAFF B-cell activating factor

BAFFR B-cell activating factor receptor

BBB Blood-brain barrier

Bcl-2 B-cell lymphoma 2

BCMA B-cell maturation antigen

BCR B cell receptor

Breg Regulatory B cells

BTK Bruton's Tyrosine Kinase

CCL C-C motif ligand

CCR C-C motif receptor

CCR7 Receptor c-c chemokine receptor 7

CD40L CD40 ligand

CFSE Carboxyfluorescein succinimidyl ester

CIS Clinically isolated syndrome

CNS Central nervous system

CONFIRM Comparator and an Oral Fumarate In RRMS

CSF Cerebrospinal fluid

CSM Class-switched memory

CVID Common variable immunodeficiency disease

CXCL C-X-C motif ligand

CXCR C-X-C motif receptor

DEFINE Determination of the Efficacy and Safety of Oral Fumarate in RRMS

DMD Disease-modifying drugs 


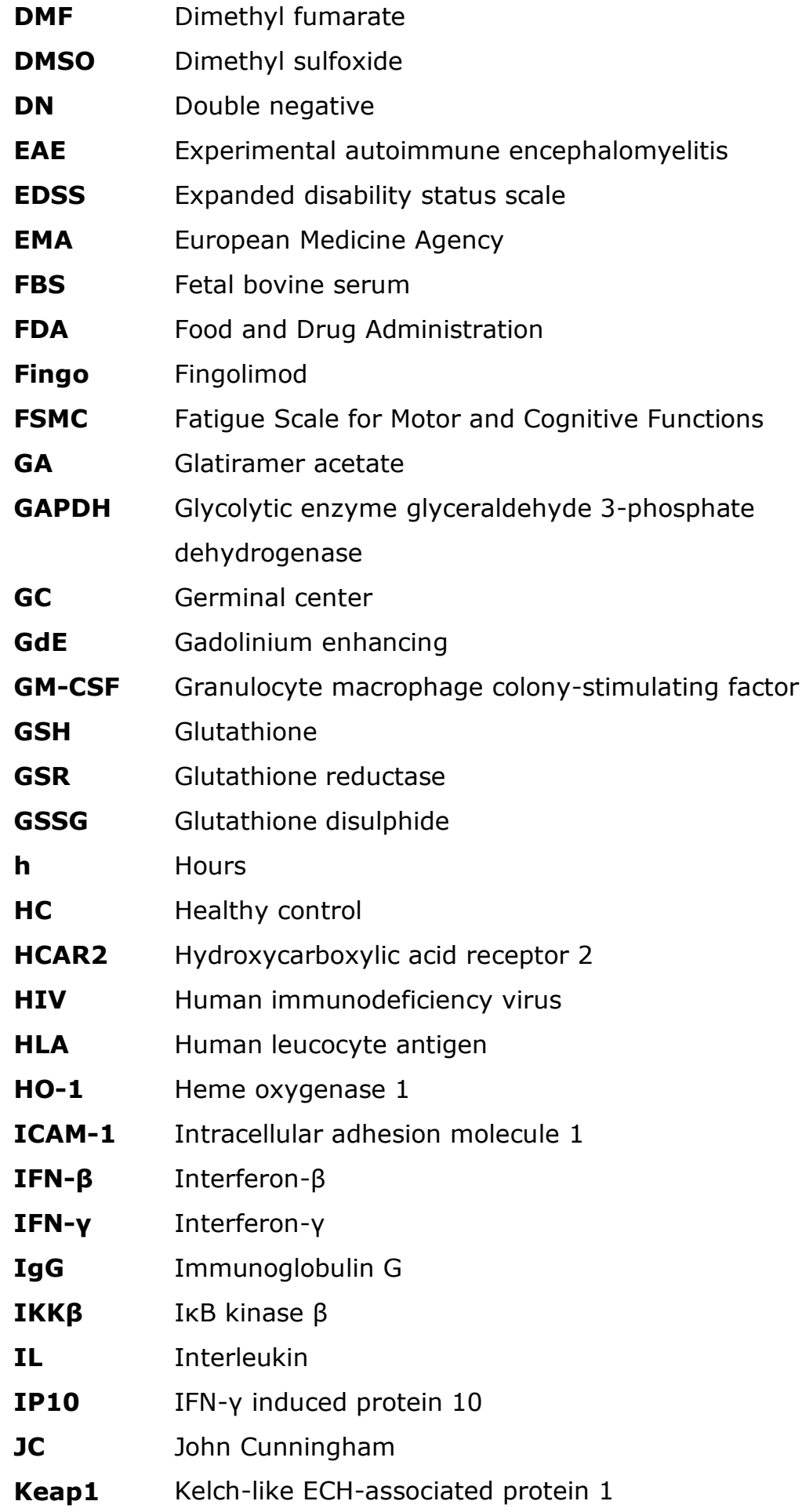

Keap1 Kelch-like ECH-associated protein 1 
LFA-1 Lymphocyte function-associated antigen-1

LLN Lower limit of normal

LT-a Lymphotoxin-a LT-a

m Months

MBP Myelin basic protein

MFI Mean fluorescence intensity

MHC-II Major histocompatibility complex - II

MIP-1 $\beta \quad$ Macrophage inflammatory protein $1 \beta$

MMF Monomethyl fumarate

MOG Myelin oligodendrocyte glycoprotein

MRI Magnetic resonance imaging

MS Multiple sclerosis

MSFC Multiple Sclerosis Functional Composite

N.A. Not applicable

NADP Nicotinamide adenine dinucleotide phosphate

NCSM Non class-switched memory

NF- $\mathbf{k B} \quad$ Nuclear factor (NF)-KB

NHPT Nine Hole Peg test

NK Natural killer

NQO-1 Quinoline oxidoreductase-1

Nrf2 Nuclear factor erythroid-derived 2

OCB Oligoclonal immunoglobulin $\mathrm{G}$ bands

PASAT- Paced Auditory Serial Addition Test (PASAT-3")

3"

PBMC Peripheral blood mononuclear cells

PF Proliferation factor

PKC- $\boldsymbol{\theta}$ Protein kinase $\mathrm{C}$ theta

PLP Proteolipid protein

PMA Phorbol 12-myristate 13-acetate

PML Progressive multifocal leukoencephalopathy

PPMS Primary progressive multiple sclerosis

PS Phospatidylserine

RA Rheumatoid arthritis

ROS Reactive oxygen species 
RRMS Relapsing-remitting multiple sclerosis

s Seconds

S1PR Sphingosine-1-phosphate receptor

SD Standard deviation

SLE Systemic lupus erythematosus

SNPs Single-nucleotide polymorphisms

SPAG16 Sperm-associated antigen 16

SPMS Secondary progressive multiple sclerosis

T25FW Timed 25-foot walk

TACI Transmembrane activator and calcium modulator and cyclophilin ligand interactor (TACI)

T-bet T-box transcription factor

TCR T cell receptor

TF Teriflunomide

Th T helper

TLR Toll-like receptor

TNF-a Tumor necrosis factor-a

Treg Regulatory T cells

UT Untreated

VCAM-1 Vascular cell adhesion molecule

VLA-4 Very late antigen-4 



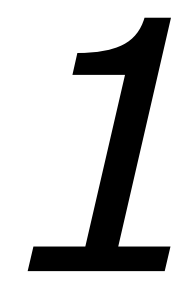

\section{Introduction and aims}

Based on:

Dimethyl fumarate treatment in multiple sclerosis: recent advances in clinical and immunological studies

Gwendoline Montes Diaz ${ }^{1}$, Raymond Hupperts ${ }^{2,3}$, Judith Fraussen ${ }^{1 *}$, Veerle Somers ${ }^{1 *}$

Autoimmunity reviews, 2018

* Equally contributing authors 


\section{Introduction and aims}

\subsection{Multiple sclerosis}

\subsubsection{Clinical aspects of MS}

Multiple sclerosis (MS) is a chronic inflammatory disorder of the central nervous system (CNS) in which infiltrated autoreactive immune cells damage the myelin and surrounding axons resulting in a chronic demyelinating and neurodegenerative disease. In the Western world, MS is the most common cause of non-traumatic neurological impairment and disability amongst young adults (1). MS is generally diagnosed between 20 and 40 years of age, and it has a female over male preponderance of $3: 1$ (2-6). The disease affects approximately 2.3 million people worldwide and until now, no cure is available $(1,2,4)$. The clinical course of MS is generally categorized as either primary progressive (PPMS), relapsing-remitting (RRMS) or secondary progressive (SPMS). In $85 \%$ of the MS patients, the disease emerges as a clinically isolated syndrome (CIS), characterized by one white matter lesion that may cause an acute or subacute episode of neurological disturbance, where the diagnostic criteria for MS are not yet met. RRMS is the most common form of MS, affecting $85-90 \%$ of all MS patients, in which periods of clinical relapses alternate with periods of remission $(1,7,8)$. Previously, $90 \%$ of untreated RRMS patients were shown to develop progressive disability, with or without superimposed relapses, described as SPMS, within 25 years $(9,10)$. However, the advent of new disease modifying treatments has led to a decrease in progression, with 15 - 30\% of RRMS patients progressing to SPMS over a long-term follow-up nowadays $(4,11)$. PPMS is diagnosed in $15 \%$ of MS patients that show progressive disability from disease onset without relapsing periods, causing irreversible damage (12).

As different neuroanatomical locations are affected in MS, a wide range of clinical symptoms might appear. One of the most common symptoms of MS is spasticity (25-65\% of MS patients). Spasticity limits patients to participate in daily activity which can be due to restricted mobility, muscle stiffness, muscle weakness and involuntary sudden movements (spams) (13-17). Mild to severe motor weakness and dysfunction is prevalent in $50 \%$ of MS patients (15). Motor dysfunction is experienced as ataxia/tremor in $46,8 \%$ of MS patients which can cause MS patients to lose ability of fine motoric skills (e.g. drinking from a cup) and to have 
an unsteady gait $(14,18)$. Furthermore, other motor dysfunctions include gait disability ( $25 \%-82 \%$ of MS patients) (17) and disturbed balance (22\% of MS patients), causing walking difficulty in MS patients (15). Another frequent symptom is fatigue, which is experienced in $75 \%$ of MS patients, this symptom is perceived persistently or sporadically $(17,19)$. Cognitive impairment which can cause defects in verbal memory, abstract reasoning, linguistic processes and deficits in attention/short-term spatial memory is reported as one of the symptoms in $26 \%$ - $56 \%$ of the MS patients (20). The prevalence of the symptoms can vary and depend on the phenotype of MS, the disease duration, EDSS and treatment. For instance, patients with a low EDSS $(\leq 3)$ coupled with lengthy disease duration ( $\geq 15$ years) remain fully ambulatory and have a good physical health, however these MS patients experience symptoms such as fatigue (49\%) and cognitive impairment $(45 \%)(21,22)$. Furthermore, MS patients with a low EDSS ( $\leq 3$ ) also experience mild physical disability while fatigue was reported as an issue by $95 \%$ of patients and cognitive difficulties were reported by $71 \%$ of patients (23). This causes MS to be a heterogeneous disease in terms of clinical symptoms, disease course and disease severity $(1,24)$. Earlier natural history studies found that within 20 years of diagnosis, $50 \%$ have a progression in disability and require assistance to walk (cane, crutch or wheelchair) $(1,25)$. However due to new disease modifying treatments only $11.3 \%$ (26) and $50 \%$ (11) of the RRMS patients have developed SPMS after a median time of 16.8 years and 32 years since disease onset, respectively.

MS adversely affects the quality of life, accompanied by frequent unemployment and social isolation and, given its high rate of disability and fairly early onset, it is associated with high costs for MS patients and society as a whole (23, 27-29). MS is thought to have a multi-causal character, in which a combination of genetic and environmental risk factors are involved (1). Large-scale epidemiological studies have identified that prior infection with certain viruses (e.g. Epstein-Barr virus and cytomegalovirus), cigarette smoke, higher latitude and geographical habitation (north and south of the Equator), vitamin D deficiency, diet and obesity in early life can increase the risk of developing MS (4, 30-37). Considering genetic predisposition, single-nucleotide polymorphisms (SNPs) in different types of human leucocyte antigen (HLA) genes have the strongest association with MS, particularly the HLA-DR15 and DR16 gene (4, 38-41). These genes encode antigen 
presentation molecules that are responsible for the presentation of antigens to $T$ cells (38-40). In addition, in different genome-wide association studies, alterations in different immunologically relevant genes are associated with MS, for instance, genes encoding interleukin (IL)-7 and IL-2 receptors as well as genes associated with costimulatory molecules (e.g. CD80, CD86, CD40) are identified as MS susceptibility genes $(38-40,42,43)$. These costimulatory molecules are essential for $\mathrm{T}$-cell dependent immunoglobulin class switching, $\mathrm{T}$ cell activation, germinal center formation and memory B cell development (43-45). In addition, rare variants in risk gene Fc receptor-like (FCRL) were found in $B$ cells of MS patients. FCRL gene family function as regulators of proliferation in B cells (43, 46). Another MS risk gene that influences $B$ cell biology is regulator of $G$ protein signaling 1 (RGS1). MS patients carrying RGS1 rare variants show decreased expression of RGS1 in B cells and this could foster a more active phenotype which eases their ability to migrate to the CNS (47).

\subsubsection{Diagnosis of MS}

The diagnosis of MS is based on clinical symptoms, neurological examination by means of imaging and laboratory evidence. Lesions and abnormalities in the white matter can be visualized by magnetic resonance imaging (MRI). According to the 2017 revisions of the McDonald criteria, clinically definite MS is diagnosed in patients that have repeated clinical attacks (disease dissemination in time) in distinct anatomical locations within the CNS (disease dissemination in space). Furthermore, cerebrospinal fluid (CSF) analysis shows elevated oligoclonal immunoglobulin $\mathrm{G}$ (IgG) bands (OCB) in more than $90 \%$ of MS patients (48). Due to the revisions, the presence of OCB could confirm the diagnosis of MS in CIS patients who do not meet criteria for disease dissemination in time, but have met the criteria for dissemination in space (49). Additionally, recording evoked potentials in afferent and efferent pathways of the CNS allows the evaluation of demyelination and axonal loss (50).

\subsubsection{Immunopathogenesis of MS}

MS pathogenesis is featured by autoimmune responses targeted toward the CNS. Adaptive immune cells, consisting of both $\mathrm{T}$ and $\mathrm{B}$ cells are currently designated as the key players in the pathogenesis of MS $(1,3,4)$. The most generally accepted hypothesis (extrinsic) traditionally starts with the activation and 
expansion of autoreactive $\mathrm{T}$ cells that have escaped tolerance checkpoints, via interaction with antigen-loaded dendritic cells in the periphery (Figure 1.1) (51, 52). These autoreactive $\mathrm{CD}^{+}{ }^{+} \mathrm{T}$ cells are most likely activated by molecular mimicry, bystander effects or cross-reactivity in the periphery (53-55). Autoreactive lymphocytes disrupt the blood-brain barrier (BBB) and acquire access to the CNS where they are reactivated by antigen presenting cells (Figure 1.1). This leads to the production of inflammatory mediators by $\mathrm{T}$ cells and microglia such as cytokines and chemokines, which in turn results in the recruitment of other immune cells such as $\mathrm{B}$ cells, $\mathrm{CD} 8^{+} \mathrm{T}$ cells and macrophages to the CNS. Infiltrated immune cells will cause an inflammatory reaction that leads to demyelinated lesions throughout the CNS (Figure 1.1) $(3,55)$. This theory was reinforced in experimental autoimmune encephalomyelitis (EAE), an animal model of $\mathrm{MS}$, in which adoptive transfer of myelin reactive $C D 4^{+} \mathrm{T}$ cells generated a $\mathrm{T}$ cell mediated destruction of the myelin sheath (56). Recent clinical observations suggest a more prominent role for B cells in this model. However, their exact function in the initiating processes is not yet clear. Next to the extrinsic hypothesis describing that the disease is triggered in the periphery, the intrinsic hypothesis believes that the pathological processes start in the CNS by the release of CNS proteins into the periphery, with the activation and infiltration of autoreactive lymphocytes occurring as a secondary event (57). In both hypotheses, demyelination will result in the formation of sclerotic plaques astrocytosis, oligodendrocyte depletion and neuronal and axonal degeneration (Figure 1.1) (1, 3). RRMS is characterized by clearly defined attacks (relapses) that are caused by the inflammatory response in the CNS, as described here. Relapses are defined by a duration of at least 24 hours and can last for months, after which they are partially or completely resolved (remission) $(1,3)$. During remission, the inflammatory cells that reside in the MS plaque, undergo activation-induced cell death, which is partly induced by regulatory cells. The resolution of inflammation is followed by remyelination (58).

\subsubsection{Players in MS}

A key component in the pathogenesis of MS is a disrupted balance between proinflammatory and anti-inflammatory immune responses favouring proinflammatory responses. In the traditional view of MS, T cells were considered as the central players in MS pathogenesis. However, in the updated view of MS, 
results of B cell depleting therapies in MS highlight a more central role of B cells in MS pathogenesis (59-62). Systemic inflammatory responses in MS patients involve cascades of aberrant activation of autoreactive naive $T$ and $B$ cells in the periphery resulting in the expansion of (effector) memory $T$ and $B$ cells, considered as the pathological cell subtypes that contribute to MS pathophysiology (62). After activation, naive autoreactive $\mathrm{CD}^{+} \mathrm{T}$ cells differentiate into different effector $\mathrm{T}$ cells with distinct phenotypes and effector functions (63). Naive T cells can differentiate into $\mathrm{T}$ helper 1 (Th1) cells, which produce IFN- $\mathrm{Y}$, or $\mathrm{T}$ helper 17 (Th17) cells, which produce interleukin-17 (IL-17). At first, MS was viewed to be a Th1 mediated disease, however, currently it is believed that it is a combined IFN-y and IL-17 driven disease $(63,64)$. Furthermore, granulocyte macrophage colony-stimulating factor (GM-CSF), which is produced by Th cells, is thought to play a role in the maintenance of autoimmune neuroinflammation in MS (65). After antigen exposure and subsequent expansion, a fraction of primed $\mathrm{T}$ cells become memory $\mathrm{T}$ cells $\left(\mathrm{CD} 45 \mathrm{RO}^{+} \mathrm{CD} 45 \mathrm{RA}^{-}\right)$that respond faster upon secondary challenge with the same antigen and give a qualitatively different and quantitatively enhanced response (63). Two main types of memory $T$ cells exist, central and effector memory $T$ cells (66). Central memory $T$ cells express the homing receptor $\mathrm{c}-\mathrm{c}$ chemokine receptor 7 (CCR7) and are able to home to secondary lymphoid organs where they provide help to $B$ cells and stimulate dendritic cells upon secondary antigenic challenge but they lack effector functions. Effector memory $T$ cells have lost the homing CCR7 expression, secrete proinflammatory cytokines such as interferon- $y$ (IFN- $y$ ) and migrate to the CNS via high expression levels of integrins, chemokine and tissue-specific homing receptors (66).

One important B cell function in MS is the production of autoantibodies by plasma cells (67). Further, MS exacerbations are characterized by an increased frequency of memory $B$ cells $\left(C D 27^{+}\right)$, which are considered to be main drivers of disease because of their pro-inflammatory cytokine production and activation of T cells via antigen presentation and costimulation $(68,69)$. Double negative (DN, IgD-CD27) B cells, that showed antigen-experienced Ig genes, were increased in a proportion of MS patients and expressed pro-inflammatory cytokines and functional molecules involved in the induction of $T$ cell responses $(68,70)$. Additionally, cytotoxic T cells $\left(\mathrm{CD}^{+}\right)$are major players in $\mathrm{MS}$, in particular as pro- 
inflammatory cells containing cytolytic granules and producing granzyme B and perforin (71). In post-mortem MS brain lesions, cytotoxic T cells outnumbered $\mathrm{CD}^{+} \mathrm{T}$ cells and their abundance was correlated with axonal injury (72-74).

Responses of adaptive immune cells which do not directly contribute to MS pathology, are underrepresented in MS patients. These include naive T and B cells, $\mathrm{T}$ helper 2 (Th2) cells, producing IL-4, and regulatory $\mathrm{T}$ and $\mathrm{B}$ cells (Treg and Breg), producing IL-10. In MS patients, Treg (CD4 $\left.{ }^{+} \mathrm{CD} 25^{+} \mathrm{CD} 127^{\text {low }}\right)$ have a reduced potential to inhibit the proliferation of effector $T$ cells compared to Treg of healthy controls $(\mathrm{HC})(75,76)$. Furthermore, Breg of MS patients produced less IL-10 than Breg cells of HC (77). This failure of Treg and Breg to suppress autoreactive $\mathrm{T}$ and $\mathrm{B}$ cells is, to a certain extent, the underlying cause of the immune dysregulation observed in MS patients (75-77).

Besides the adaptive immune system, the innate immune system is increasingly implicated in MS. Natural killer (NK) cells can be divided into CD56 ${ }^{\text {dim }}$ CD $16^{+}$NK cells and CD56 ${ }^{\text {bright }} \mathrm{CD} 16^{\mathrm{dim}} \mathrm{NK}$ cells (78). In MS, the disruption of the killing activity of NK cells was correlated with symptomatic relapse (79). Furthermore, CD56 bright $\mathrm{NK}$ cells showed the capacity to suppress the proliferation of $\mathrm{CD}^{+}{ }^{+} \mathrm{T}$ cells (80-82). Classical monocytes $\left(\mathrm{CD} 14^{+} \mathrm{CD} 16^{-}\right)$are described as pathological effector cells in MS as they produce pro-inflammatory factors and reactive oxygen species (ROS), and act as phagocytes $(83,84)$. In contrast, patrolling monocytes $\left(\mathrm{CD} 14^{+} \mathrm{CD} 16^{+}\right)$are considered to be anti-inflammatory because of a reduced ROS production and phagocytosis $(83,84)$. However, patrolling monocytes have also been described as pro-inflammatory due to their high production of proinflammatory cytokines and ability to activate $T$ cells and induce their migration to the CNS (83-85). 
Secondary lymph nodes

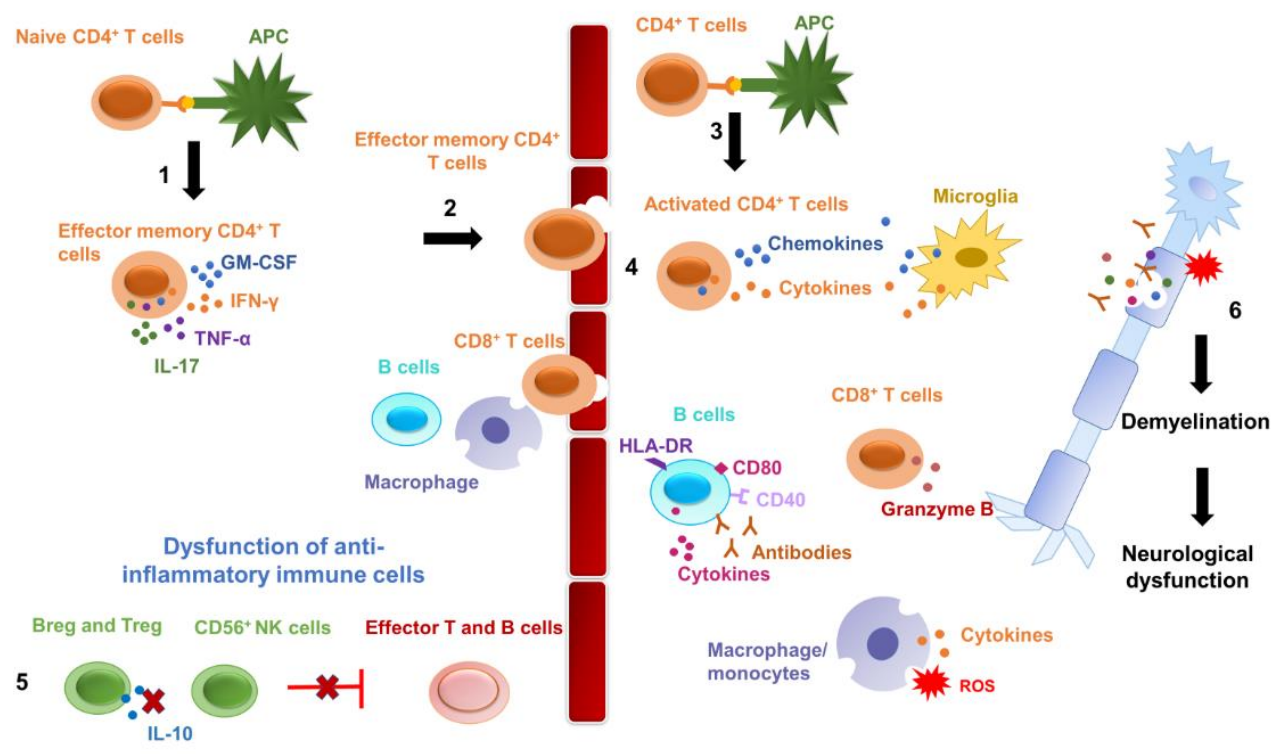

Blood-brain barrier

Central nervous system

Figure 1.1 Pathogenesis of MS. (1) Naive autoreactive CD4 ${ }^{+} \mathrm{T}$ cells differentiate into effector (memory) T cells after antigen-presentation by antigen presenting cells (APC). (2) Effector $\mathrm{CD}^{+} \mathrm{T}$ cells migrate through the disrupted blood-brain barrier (BBB) into the CNS. (3) $\mathrm{CD}^{+} \mathrm{T}$ cells are reactivated by antigen presenting cells in the CNS. (4) Reactivated $\mathrm{CD} 4^{+} \mathrm{T}$ cells and microglia secrete chemokines and cytokines. B cells, CD8 ${ }^{+} \mathrm{T}$ cells and macrophages/monocytes are recruited from the periphery into the CNS by inflammatory mediators. In the CNS, B cells exert pro-inflammatory functions such as antigen presentation (HLA-DR), costimulation (CD80, CD40) and secrete cytokines and antibodies. CD8 ${ }^{+} \mathrm{T}$ cells produce pro-inflammatory granzyme $B$ and macrophages/monocytes secrete cytokines and reactive oxygen species (ROS) in the CNS. (5) Functional capacities of anti-inflammatory immune cells such as IL-10 secretion or inhibition of effector T and B cells by Breg, Treg and $\mathrm{CD} 6^{+} \mathrm{NK}$ cells is disrupted. (6) Inflammatory mediators produced by infiltrated immune cells attack the myelin sheet which results in demyelination and neurological dysfunction. $\mathrm{APC}=$ antigen presenting cell, GM-CSF = granulocyte macrophage colony-stimulating factor, IFN- $y=$ interferon- $y$, TNF- $a=$ tumor necrosis factor- $a$, IL-17 = interleukin-17, Breg = regulatory $B$ cells, Treg $=$ regulatory $T$ cells, $I L-10=$ interleukin $10, H L A-D R=$ human leukocyte antigen-DR, ROS = reactive oxygen species. 


\subsection{B cells in MS}

The striking ability of anti-CD20 monoclonal antibodies to limit new MS relapses have forced the MS society to update the conceptual framework of MS immunopathophysiology (62). In order to do this, more attention needs to be given to the potential contribution of B cells in MS. In this part, an overview of the contribution of the different B cell subtypes to MS pathology and their pathological antibody-dependent and more importantly antibody-independent functions in the disease activity of MS is provided.

\subsubsection{B cell subtypes}

B cell development starts in the bone marrow, where hematopoietic stem cells differentiate into immature $B$ cells $\left(\mathrm{CD} 19^{+} \mathrm{IgM}^{+}\right)$. Immature $B$ cells then enter the circulation as transitional B cells $\left(\mathrm{CD} 19^{+} \mathrm{CD} 27^{-} \mathrm{CD} 38^{+}\right)$and differentiate into naive $B$ cells $\left(C D 19^{+} \mathrm{IgD}^{+} \mathrm{CD}^{2} 7^{-}\right.$) (Figure 1.2). After encountering their specific antigen in the secondary lymphoid organs, naive B cells can differentiate into short-lived plasma blasts $\left(\mathrm{CD} 19^{+} \mathrm{CD} 138^{++}\right.$or $\left.\mathrm{CD} 19^{+} \mathrm{CD} 27^{+} \mathrm{CD} 38^{++}\right)$or plasma cells $\left(\mathrm{CD} 19^{+/-}\right.$ $\mathrm{CD} 8^{+} \mathrm{CD} 138^{+}$) that secrete low-affinity autoantibodies for a short period of time. Alternatively, activated $B$ cells differentiate into memory B cells $\left(C D 19^{+} C D 27^{+}\right)$, plasma blasts or long-lived plasma cells in a germinal center reaction. Long-lived plasma cells secrete high-affinity autoantibodies for a longer period of time. Memory B cells can be divided into non class-switched memory B cells (NCSM, $\left.\mathrm{CD}^{19}{ }^{+} \mathrm{IgD}^{+} \mathrm{CD}_{27}{ }^{+}\right)$and class-switched memory B cells (CSM, CD19+IgD $\left.{ }^{-} \mathrm{CD} 27^{+}\right)$ that have undergone isotype switching to IgG, IgA or IgE expression. However, a small number of memory B cells preserve IgM expression and are known as IgM only memory B cells (CD19+ ${ }^{+} \mathrm{gD}^{-} \mathrm{CD} 27^{+} \mathrm{IgM}^{+}$) (Figure 1.2). CSM and NCSM B cells can further differentiate into plasma cells $\left(\mathrm{CD} 19^{+} \mathrm{CD} 38^{+} \mathrm{CD} 138^{+}\right)$upon a second challenge with their specific antigen $(86,87)$. Another $B$ cell subtype is made up by the DN B cells (CD19+IgD-CD27-), of which the origin is still under debate (Figure 1.2). Although DN B cells are negative for the memory marker CD27, they show similarities with $\mathrm{CD} 27^{+}$CSM B cells. The majority of DN B cells are $\mathrm{IgG}^{+}$, have shortened telomeres (88) and express mutated Ig genes, which suggests that they were selected by an antigen-driven process similar to $\mathrm{CD}^{2} 7^{+} \mathrm{IgG}^{+}$ memory B cells (70). Moreover, it is suggested in healthy subjects that a clonal 
relationship between DN and $\mathrm{CD} 27^{+}$memory B cells exists (89). Furthermore, DN B cells are able to produce pro-inflammatory cytokines $(68,90)$.

MS patients present with a dysregulated immune system showing an overrepresentation of memory $B$ cells and DN B cells and underrepresentation of naive, transitional $B$ cells and Breg $\left(C D 19^{+} C D 24^{++} C D 38^{++}\right)(68,77,91,92)$. Breg are considered to be anti-inflammatory via the production of IL-10 and direct inhibition of activated $\mathrm{T}$ and $\mathrm{B}$ cells $(77,93,94)$. They are enriched from transitional B cells or plasma cells (Figure 1.2) (95). 


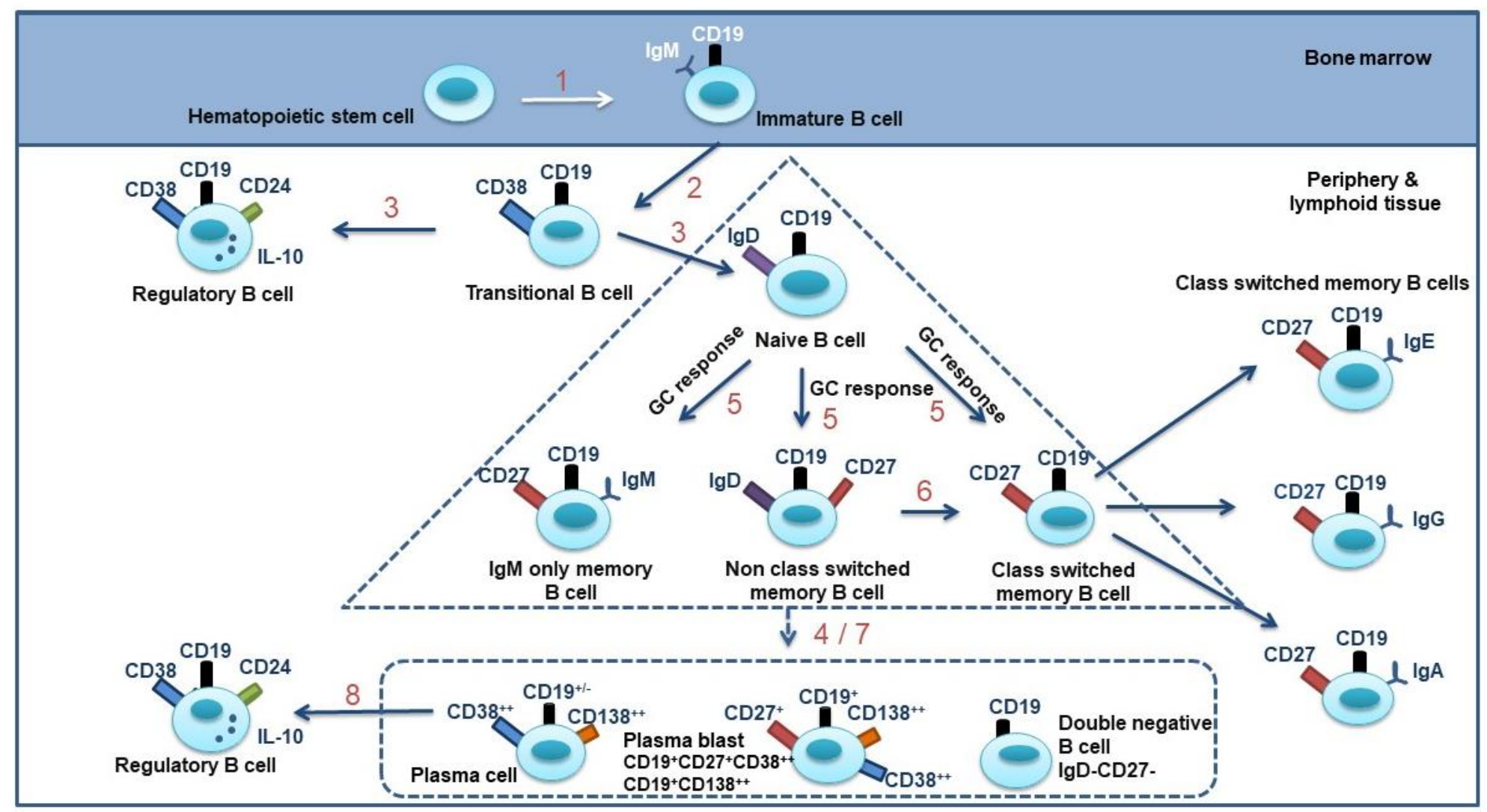


Figure 1.2. B cell subtypes. (1) Hematopoietic stem cells differentiate into immature $\mathrm{B}$ cells $\left(\mathrm{CD} 19^{+} \mathrm{IgM}^{+}\right)$in the bone marrow and (2) enter the circulation as transitional $\mathrm{B}$ cells $\left(\mathrm{CD} 19^{+} \mathrm{CD} 27^{-} \mathrm{CD} 38^{+}\right)$. (3) Transitional B cells migrate to the secondary lymphoid organs and differentiate into naive $\mathrm{B}$ cells $\left(\mathrm{CD} 19^{+} \mathrm{IgD}{ }^{+} \mathrm{CD} 27^{-}\right)$or Breg $\left(\mathrm{CD} 19^{+} \mathrm{CD} 24^{++} \mathrm{CD} 38^{++}\right)$. (4) B cells remain naive until they encounter an antigen after which they differentiate into shortlived plasma cells $\left(\mathrm{CD} 19^{+} \mathrm{CD} 38^{+} \mathrm{CD} 138^{+}\right)$, plasma blast $\left(\mathrm{CD} 19^{+} \mathrm{CD} 27^{+/-} \mathrm{CD} 138^{+}\right)$or DN B cells $\left(\mathrm{CD} 19^{+} \mathrm{IgD}-\mathrm{CD} 27^{-}\right)$. (5) On the other hand, naive B cells can further mature into IgM only memory B cells $\left(\mathrm{CD} 19^{+} \mathrm{CD} 27^{+} \mathrm{IgM}^{+}\right)$, NCSM B cells $\left(\mathrm{CD} 19^{+} \mathrm{IgD} \mathrm{CD}^{+} 7^{+}\right)$, CSM B cells $\left(\mathrm{CD}_{19}{ }^{+} \mathrm{IgD}^{-} \mathrm{CD}_{27} 7^{+} \mathrm{IgA}^{+} / \mathrm{IgM}^{+} / \mathrm{IgG}^{+}\right)$in a GC response. However, NCSM B cells can also be formed independent of a GC and (6) can differentiate further into CSM B cells. (7) A proportion of the memory $B$ cells further differentiate into long-lived plasma cells, plasma blast or DN B cells after a second encounter with the same antigen. (8) Plasma cells can differentiate into Breg. Breg = regulatory $B$ cells, NCSM = non class-switched memory $B$ cells, CSM = class-switched memory $\mathrm{B}$ cells, $\mathrm{DN}=$ double negative $\mathrm{B}$ cells, $\mathrm{GC}=$ germinal center. 


\subsubsection{Pathological functions of B cells in MS}

B cells are mostly known for their differentiation into antibody-producing plasma cells. The use of B cell depletion therapy, including the anti-CD20 monoclonal antibodies rituximab and ocrelizumab, has indicated that B cells also exert an antibody-independent function in the pathology of MS. Even though antibodysecreting plasma cells do not express CD20 and therefore cannot be targeted by these therapies, it is shown in clinical trials that anti-CD20 monoclonal antibody therapy reduced brain lesions and clinical relapses in MS patients (96-98). These positive outcomes were not the result of decreased antibody titers, but suggested other functions for B cells in MS $(99,100)$.

\section{Antibody producing function of B cells}

Different targets of autoreactive antibodies in MS have been identified over the past decades. Early pathological studies of perivascular MS injury have identified antibodies bound to myelin peptides within phagocytic cells $(67,101)$. Via the use of in vitro models, it is suggested that IgG antibodies of MS patients can cause neuronal degeneration by activation of the complement system and antibodydependent cell-mediated cytoxicity $(86,102-106)$. OCB and an elevated antibody synthesis rate in the CSF of MS patients are indicators of abnormal antibody production within the CNS $(107,108)$.

Different antibody targets have been described in MS, including myelin basic protein (MBP), myelin oligodendrocyte glycoprotein (MOG), proteolipid protein (PLP), neurofilament, sperm-associated antigen 16 (SPAG16), chloride-channel protein anoctamin 2 (ANO2), potassium channel KIR 4.1 and other components of the CNS, emphasizing the complex antibody responses in MS (109-111). In addition, the presence of cells secreting anti-MBP or anti-PLP autoantibodies was correlated with intrathecal IgG, IgA, and IgM synthesis (112). 
1. Autoreactive antibody production CSF Plasma SPAG16 ANO2 KIR 4.1 MBP PLP MOG

2. Pro-inflammatory cytokine secretion

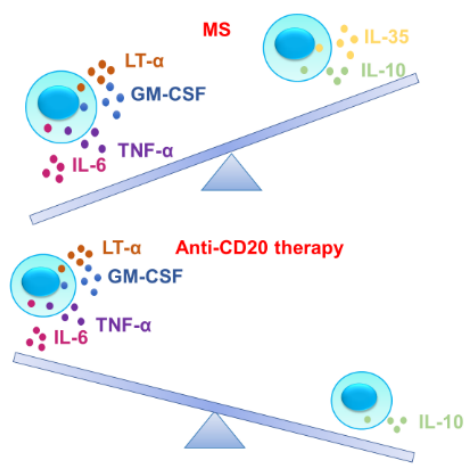

3. Antigen presentation and T cell activation
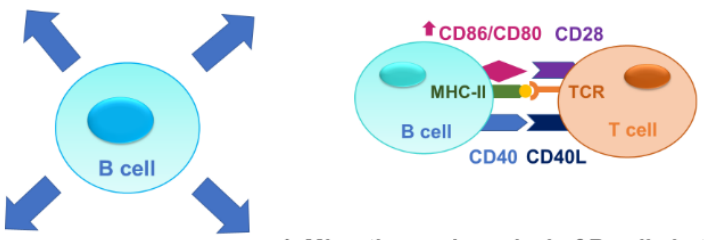

4. Migration and survival of $B$ cells in the CNS

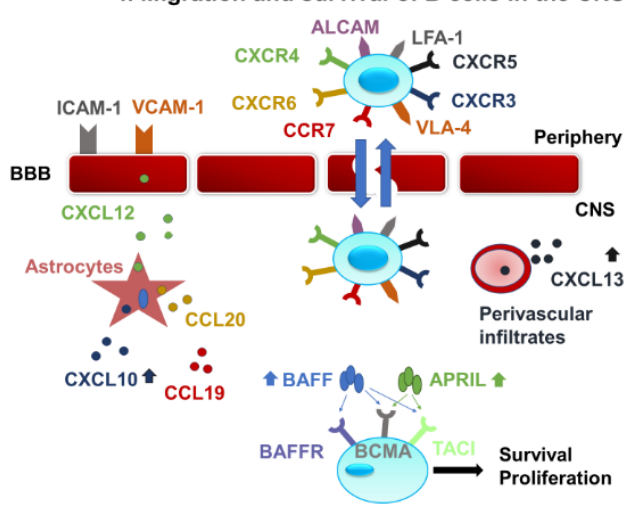

Figure 1.3 B cell functions in MS. (1) plasma cells of MS patients produce autoreactive antibodies. Antibodies directed against different antibody targets (SPAG16, ANO2, KIR 4.1, MBP, PLP, MOG) have been found in the CSF and/or plasma of MS patients. (2) B cells of MS patients produce less anti-inflammatory cytokines (IL-10 and IL-35) and more pro-inflammatory cytokines (lymphotoxin-a (LT-a), GM-CSF, tumor necrosis factor-a (TNF-a), IL-6). B cell depletion therapy in MS patients shifts the balance back to a more anti-inflammatory profile. (3) B cells of MS patients present antigens via the major histocompatibility complex - II (MHC-II) molecule to T cells. B cells of MS patients will further activate $T$ cells via costimulatory molecules CD86 and CD80. The expression of CD86 and CD80 is increased on B cells of MS patients. B cells get activated via binding of CD40 to CD40 ligand (CD40L) on T cells which induces class-switching of B cells (4) B cells of MS patients are attracted from the periphery to the CNS via the expression of chemokine C-XC motif (CXC) ligand 13 (CXCL13), CXCL10, CXCL12, C-C motif (CC) ligand 20 (CCL20) and CCL19 by astrocytes, blood vessels and perivascular infiltrates, which bind the chemokine receptors CXC receptor 5 (CXCR5), CXCR3, CXCR4, CC receptor 6 (CCR6) and CCR7 of B cells, respectively. The migration of $B$ cells of MS patients is preceded by the following steps: rolling, adhesion (very late antigen-4 (VLA-4) on B cells binds vascular cell adhesion molecule (VCAM-1) on BBB), crawling (lymphocyte function-associated antigen-1 (LFA-1) on $B$ cells bind intracellular adhesion molecule 1 (ICAM-1) on BBB) and transmigration, which depends on activated leukocyte cell adhesion molecule (ALCAM) expression on B cells. $B$ cells of MS patients survive and proliferate in the CNS due to the increased levels of B-cell activating factor (BAFF) and a proliferation-inducing ligand (APRIL) in the CNS that both 
bind to B-cell maturation antigen (BCMA) and transmembrane activator and calcium modulator and cyclophilin ligand interactor (TACI) on B cells. The BAFF receptor (BAFFR) on B cells only binds BAFF. CSF = cerebrospinal fluid, SPAG-16 = sperm-associated antigen 16, ANO 2 = anoctamin 2, KIR 4.1 = potassium channel KIR 4.1, MBP = myelin basic protein, $\mathrm{PLP}=$ proteolipid protein, $\mathrm{MOG}=$ myelin oligodendrocyte glycoprotein, $\mathrm{MS}=$ multiple sclerosis , GM-CSF = granulocyte macrophage colony-stimulating factor, $\mathrm{TCR}=\mathrm{T}$ cell receptor, $\mathrm{CNS}=$ central nervous system, $\mathrm{BBB}=$ blood-brain barrier.

\section{Cytokine secreting function of B cells}

Another important pathological role of B cells in MS patients is the secretion of pro-inflammatory cytokines. In vitro experiments have indicated that $\mathrm{B}$ cell activation via $B$ cell receptor (BCR) cross-linking followed by a costimulatory CD40 ligand (CD40L) signal results in the production of the pro-inflammatory cytokines lymphotoxin-a (LT-a), tumor necrosis factor-a (TNF-a), GM-CSF and IL-6 (100, $113,114)$. When only CD40L was used for B cell activation, the anti-inflammatory cytokines IL-10 and IL-35 were produced (100, 113, 114). In MS patients, the balance between pro-inflammatory and anti-inflammatory B cell cytokines is disrupted and B cells of MS patients produce less IL-10 and secrete more IL-6, TNF-a, LT-a and GM-CSF than B cells of HC (100, 114-117). B cell depletion following anti-CD20 monoclonal antibody therapy in MS patients was shown to coincide with decreased inflammatory $\mathrm{T}$ cell responses in a way that was partly dependent on the elimination of B cells producing LT-a, TNF-a and IL-6 (100, 115, 116). Furthermore, B cell depletion in MS patients resulted in a decreased proinflammatory myeloid cell response that is dependent on the elimination of GMCSF-producing B cells $(100,115,116)$. After B cell depletion therapy, the reconstituting $B$ cell population consists mostly of naive $B$ cells that produce less pro-inflammatory cytokines and higher amounts of IL-10. This reconstituted $B$ cell population is associated with a persistent decrease in T cell and myeloid-lineage pro-inflammatory responses in MS patients $(100,115,116)$.

\section{The role of $B$ cells as antigen presenting cells and their role in $\mathbf{T}$ cell activation}

B cells are efficient antigen presenting cells (APCs) and compared to other APCs such as dendritic cells which present linear epitopes, B cells can additionally recognize conformational epitopes by the $B C R$. After antigen recognition, the $B$ 
cell will internalize, process and present intact myelin antigens as a complex with the major histocompatibility complex (MHC)-I or II molecules such as HLA-A/B/C and HLA-DR/DP/DQ, respectively $(62,118-120)$. B cells are the main source of APCs when antigen levels are low (120-122). After antigen internalization by the $\mathrm{BCR}, \mathrm{B}$ cells are activated and the expression of co-stimulatory molecule CD86 is induced; a process that is needed for T cell activation.

Subsequently, B cells will process the protein antigen and present the peptide antigen on a MHC molecule to T cells in the secondary lymphoid organs. Upon antigen recognition by the $\mathrm{T}$ cell, a costimulatory signal is given via binding of CD86 on the B cells to CD28 on the T cells. These signals will induce CD40 engagement by CD40L of T cells, which will stabilize CD86 expression, increase MHC molecules and induce costimulatory molecule CD80 which leads to activation and pro-inflammatory differentiation and expansion of antigen-specific $T$ cells (62, $120,122)$. In the context of MS, it has been shown that mice with a B cell specific depletion of MHC-II are resistant to MOG-protein induced $E A E$, which suggests the need of $B$ cell antigen presentation in the induction of disease in this EAE model (121). Furthermore, the APC function of MOG-specific B cells is sufficient to cooperate with MOG-specific T cells in order to develop spontaneous EAE in mice with MOG-specific BCR and TCR (121). More importantly, B cells of MS patients can induce pro-inflammatory T cells in response to MBP (123). The expression of costimulatory molecules CD80 and CD86 on B cells, is higher in MS patients than in $\mathrm{HC}$ (123). In addition, there is a 12-fold and 2-fold increase in CD86 ${ }^{+}$and CD80+ $B$ cells in the CSF of MS patients compared with peripheral blood (123). These findings suggest that one of the mechanisms of the immediate benefit of $B$ cell depletion in MS may relate to an impaired activation of $T$ cells and $B$ cells, respectively.

\section{Migration and survival of B cells in the CNS}

Analysis of CSF B cells in MS has indicated the persistence of the same B cell clones marked by the same $B C R$ variable chain $\left(V_{H} D J_{H}\right)$ gene segments in patients over time (124). Furthermore, the same B cell and plasma cell clones can be shared among different CNS subcompartments (meninges, CSF, parenchyma). Somatic hypermutation studies have shown that identical B cell clones can be 
shared between the CNS and the periphery $(91,125,126)$. Furthermore, B cell clones were shown to traffic bidirectionally, into and out of the CNS.

$B$ cells of MS patients are recruited to the CNS through the expression of chemokine C-X-C motif (CXC) ligand 13 (CXCL13), CXCL10, CXCL12, C-C motif (CC) ligand 20 (CCL20) and CCL19 by astrocytes, blood vessels and perivascular infiltrates, which bind the chemokine receptors CXC receptor 5 (CXCR5), CXCR3, CXCR4, CC receptor 6 (CCR6) and CCR7 of B cells, respectively $(127,128)$. CSF levels of CXCL13 are increased in active RRMS patients and correlate with the number of total $B$ cells in the CSF and even more specifically with the number of CSM B cells in the CSF (129). Therefore, this chemokine seems to play a central role in B cell recruitment to the CNS. In addition, the inflammatory chemokine CXCL10 contributes to lesion development in MS and its concentration is significantly increased in the CSF of MS patients (130).

The formation of ectopic follicle-like structures has been indicated in the meninges of progressive MS patients $(60,131,132)$. Here, B cells can locally undergo maturation into antibody-producing plasma cells $(60,131,132)$. Elevated $B$ cell survival factors such as B-cell activating factor (BAFF) and a proliferation-inducing ligand (APRIL) promote the survival of CNS plasma cells (133). BAFF and APRIL are members of the TNF superfamily and regulate the maturation and function of $B$ cells. These survival factors are produced by neutrophils, monocytes, dendritic cells and T cells (134-137). At the clinical onset of MS, the level of BAFF is correlated with intrathecal IgG synthesis, suggesting a BAFF-dependent plasma cell survival in the CNS (132). In MS CSF, BAFF is produced by activated astrocytes in chronic lesions, whereby CSF levels of BAFF are correlated with disease severity (134-137). Three receptors for BAFF and APRIL exist on B cells, the BAFF receptor (BAFFR), which only binds BAFF, is the earliest receptor and is expressed on pro-B cell to memory $B$ cells. $B$-cell maturation antigen (BCMA) and transmembrane activator and calcium modulator and cyclophilin ligand interactor (TACI) can bind both BAFF and APRIL and are weakly expressed on immature B cells and their expression becomes progressively stronger during peripheral $B$ cell development. Upregulation of these factors in MS results in B cell proliferation and survival (134-138). B cell trafficking across the BBB not only depends on the response to chemokines, but it is mediated by sequential interactions including 
rolling (lose attachment), adhesion (firm attachment), crawling and transmigration (139).

The interactions of selectins on the $B$ cells with their ligands on the BBB reduces the velocity of B cells and induces rolling over the surface of the BBB $(139,140)$. The next step, adhesion of the $B$ cells with the BBB, depends on the adhesion molecule very late antigen (VLA)-4 on B cells, which binds to vascular cell adhesion molecule - 1 (VCAM-1) on the BBB $(139,140)$. VLA-4 has a major role in $B$ cell migration since blocking this adhesion molecule in vitro inhibits trafficking of B cells across human adult brain-derived endothelial cells (140). The relevance of this adhesion molecule is also illustrated by the therapeutic efficacy of the antiVLA4 monoclonal antibody natalizumab (141, 142). The adhesion molecule lymphocyte function-associated antigen (LFA)-1 is also important in the adhesion as well as in the crawling phase of the $B$ cells and interacts with intracellular adhesion molecule 1 (ICAM-1) on the BBB (139, 140). A role for LFA-1 expression in $B$ cell migration was demonstrated by inhibiting its counterpart ICAM-1 (140). In the final step B cells transmigrate, which implies that B cells traverse the BBB through the transcellular route (139). The endothelial ligand of the immunoglobulin superfamily, namely activated leukocyte cell adhesion molecule (ALCAM), is expressed on almost all B cells and is considered to be involved in the final transmigration step (139). Additionally, by blocking ALCAM, the contribution of ALCAM to B cell recruitment into the CNS was demonstrated (143).

\subsubsection{B cell aging}

A proportion of MS patients display characteristics of premature immune system aging (144). During aging, the immune system undergoes changes that lead to a decline or dysregulation of immunologic function. Advancing age is accompanied by an increase in the levels of inflammatory mediators in the absence of acute infections which leads to a chronic low-grade inflammation level known as inflamm-aging (145-147). In elderly, the bone marrow density is reduced and $B$ cell production is reduced which generates an inadequate immune response against newly encountered antigens (148-150). As a result, bacterial and viral infections increase whereas vaccine efficacy reduces (150). Nonetheless, B cell numbers remain relatively constant in elderly compared to young adults, since memory and effector antigen-experienced B cells proliferate, thereby continuously 
filling the immunological space. Thus, the peripheral B cell repertoire will undergo alterations ultimately resulting in a predominance of antigen-experienced $\mathrm{B}$ cells.

Humoral immune responses in aged humans are less robust and poorly protective, since a decreased production of $B$ cells results in reduced protective antibody responses but an increased autoantibody generation (150). A progressive decline in germinal center formation during aging results in decreased BCR diversity while clonal B cell expansion occurs, contributing to a restricted B cell repertoire (151). An accumulation of BCR mutations occurs which is unrelated to antigen affinity maturation and results in an alteration in the selection of memory B cells (152). In addition, two age-associated $B$ cell populations have been identified, namely

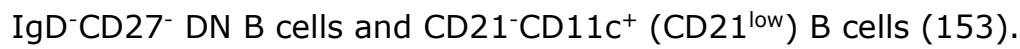

In autoimmune diseases, it is thought that chronic inflammation (i.e. repeated autoantigen stimulation) leads to alterations in immune cell phenotype and function comparable to those in normal immune aging (150). A proportion of MS patients display characteristics of premature immune aging which is thought to contribute to MS pathogenesis and progression $(144,154)$. Furthermore, an aged T cell repertoire and early thymic involution is observed in a proportion of MS patients, suggestive of a premature immune aging (144, 154-157).

DN B cell percentages were found to be increased in healthy elderly $(68,88)$ but also in subjects infected with rotavirus (158) or human immunodeficiency virus (HIV) (159), in subjects challenging Alzheimer's disease (160). In addition, the percentages of DN $B$ cells are increased in patients suffering from autoimmune diseases such as systemic lupus erythematosus (SLE) (70), rheumatoid arthritis (RA) (161) and MS (68), which are all diseases displaying chronic immune stimulation. In SLE patients, an increased percentage of DN B cells was associated with the prevalence of disease-specific autoantibodies, such as anti-double stranded DNA antibodies and a higher disease activity index (70), indicating that they may contribute to autoimmune pathology. Although DN B cells lack the memory marker $C D 27$, it is believed that they could be considered as a subtype of memory B cells since they show similarities with $\mathrm{CD} 27^{+}$CSM B cells. The DN B cells are considered heterogeneous in the expression of IgG, IgA and IgM and express BCR hypermutation which suggests that they were selected by an antigen-driven process $(70,88)$. Furthermore, the low expression of the 
transmembrane protein ATP-binding-cassette-B1 (ABCB1) transporter and their shortened telomeres indicate that they behave as $\mathrm{CD} 27^{+}$memory $\mathrm{B}$ cells, as the $A B C B 1$ transporter is only present in $C D 27^{-}$naive $B$ cells and absent in IgD ${ }^{-} C D 27^{+}$ CSM $B$ cells $(88,162)$. Moreover it is suggested that a clonal relationship between $\mathrm{DN}$ and $\mathrm{CD} 27^{+}$memory B cells exists in $\mathrm{HC}(89)$.

CD21 $1^{\text {low }} B$ cells have been described in healthy elderly and in several disorders such as HIV, Sjögren's syndrome, malaria, scleroderma, common variable immunodeficiency disease (CVID), SLE and RA $(68,163-167)$. CD21 low $B$ cells of $\mathrm{HC}$ found in tonsils seemingly belong to a novel CD27- memory subtype due to their mutated BCRs and class-switched phenotype $(168,169)$. In peripheral blood of HC, CD21 low B cells consist of a mixture of naive and memory B cells (163). However, when considering autoimmune diseases, depending on the disease and study design, the $C D 21^{\text {low }} B$ cells in peripheral blood have been described as $\mathrm{CD} 27^{+}$or $\mathrm{CD} 27^{-}$. For instance, in a study including RA and scleroderma patients $C D 21^{\text {low }} B$ cells were described as $C D 27^{+} B$ cells while in MS patients $C D 21^{\text {low }} B$ cells include $C D 27^{-}$and $C D 27^{+} B$ cells $(68,170)$. In CVID and RA, CD21 low $B$ cells produce antinuclear antibodies and displayed polyreactivity and autoreactivity (163). Moreover, they are unable to induce calcium flux after BCR triggering, suggesting that may be anergic $B$ cells that have escaped tolerance mechanisms and accumulate in the periphery of autoimmune patients (163).

An increased proportion of MS patients showed an expansion of DN and CD21 low B cells with pro-inflammatory characteristics (68). A higher proportion of MS patients younger than 60 years showed premature immune aging by an expansion of DN B cells and CD21 low $B$ cells compared to age-matched HC. In MS patients, the majority of DN B cells showed an $\mathrm{IgG}^{+}$memory phenotype, whereas $C D 21^{\text {low }}$ $B$ cells showed similar frequencies of $\mathrm{IgG}^{+}$and $\mathrm{IgM}^{+}$cells. After ex vivo stimulation, DN B cells of MS patients produced the pro-inflammatory cytokines, TNF-a, LT-a and granzyme B. Furthermore, a higher frequency of DN and CD21 low $B$ cells was found in the CSF of MS patients compared to paired peripheral blood, which suggested that these cells could exert pathological functions within the CNS (68). CD21 $1^{\text {low }} B$ cells show similarities with age-associated $B$ cells found in aged and autoimmune-prone mice $(170,171)$. 
These age-associated $B$ cells accumulate with age and are positive for CD11C, but lack the expression of CD21 and CD23 (171). Additionally, age-associated B cells depend upon the expression of the transcription factor T-bet ( $T$-Box Expressed in $\mathrm{T}$ cells). T-bet is a master regulator of the age-associated $\mathrm{B}$ cells character since overexpression of T-bet induces acquisition of the age-associated $B$ cells phenotype (172). T-bet expression can be upregulated in vitro in human peripheral blood naive $B$ cells in the presence of toll-like receptor (TLR) stimulation (TLR7 and 9), alone or together with IFN-Y and IL-21 (173). However, the functional role of T-bet expression in B cells and the consequence of this process on the immune response in autoimmune diseases remain understudied. Nonetheless, it is known that T-bet plays a role in inflammatory cytokine production and antibody isotype switching and defines an antigen-experienced $B$ cell subtype. T-bet ${ }^{+} \mathrm{B}$ cells peak during viral infections and T-bet expression promotes class-switching to IgG2a antibodies for clearing the infection (174). A conditional deletion of T-bet from B cells in lupus-prone mice demonstrated reduced kidney pathology and titers of disease-specific autoantibodies, suggesting that T-bet expressing B cells play a role in the onset and development of autoimmunity (175). In addition, the formation of autoimmune germinal centers in lupus-prone mice is driven by IFN- $\gamma$-mediated T-bet expression in B cells (176, 177). Furthermore, in human autoimmunity, increased frequencies of $\mathrm{T}^{-\mathrm{bet}^{+}} \mathrm{B}$ cells were reported in SLE, RA and scleroderma patients (178). In patients with Crohn's disease, the frequency of T-bet ${ }^{+} B$ cells was upregulated in the peripheral blood and gut of active patients and was associated with disease activity. These T-bet $^{+}$B cells were found to be a source of IFN-Y that supports intestinal inflammation and hereby contribute to the intestinal Th1 inflammation in patients with Crohn's disease (179). Thus, T-bet ${ }^{+}$B cells are present in a wide variety of human autoimmune diseases and it is critical to identify their role in the development and/or progression of autoimmunity. 


\subsection{Therapies for MS}

Before the 1990s, the treatment of MS was challenging and immune suppressive agents such as corticosteroids were the only option. Although they are effective in reducing the duration of a relapse, corticosteroids do not exert long-term neuroprotective benefits and pose an increased risk for infections and cancer (180). The treatment era for MS started in 1993, when the first injectable immunomodulating therapy, interferon $\beta$ (IFN- $\beta$ ), became available. In the past decades, several new disease-modifying drugs (DMD) were developed, with 12 DMD approved by the European Medicine Agency (EMA) in 2018 for RRMS (181). Current therapies are based on the inflammatory changes in RRMS patients, aiming to prevent disability by restoring the immune balance. This will eventually reduce, prevent or attenuate relapse rates (3). It is recommended to start treatment as early as possible, a recognition of the inflammatory process is sufficient to begin treatment with DMD even before the clinically definite diagnosis of MS (182). Delaying start of treatment to a later stage has been shown to result in a greater risk of reaching a higher disability score as measured by the Expanded Disability Status Scale (EDSS) (183). The most common treatment strategy is to start with first-line treatments, followed by second-line treatments when initial treatment doesn't work.

\subsubsection{First-line treatments}

First-line treatments have a moderate efficacy but have a high safety profile and include IFN- $\beta$, peginterferon beta-1a, glatiramer acetate (GA), teriflunomide, and dimethyl fumarate (DMF). For a long time, only injectable immunomodulating therapies, such as IFN- $\beta$ (available under trade names Avonex ${ }^{\mathrm{TM}}$, Rebif ${ }^{\mathrm{TM}}$, Betaferon $^{\mathrm{TM}}$ and Extavia ${ }^{\mathrm{TM}}$ ) and GA (Copaxone ${ }^{\mathrm{TM}}$ ), were available as first treatment options recommended for RRMS patients (3). IFN- $\beta$ treatment restores the balance of pro- and anti-inflammatory cytokines, reduces Th17 and Th1 cells and decreases the number of inflammatory cells trafficking across the BBB (184). Furthermore, IFN- $\beta$ is effective in reducing the relapse rate by $33 \%$ in MS patients and has anti-viral and anti-tumor activity $(185,186)$. Nonetheless, it has been shown that the therapeutic efficacy of IFN- $\beta$ reduces over time due to the production of neutralizing antibodies against IFN- $\beta$ (187). 
More recently, a polyethylene glycol was conjugated to IFN- $\beta$ in the new formulation peginterferon beta-1a (Plegridy ${ }^{\mathrm{TM}}$ ). Hereby, the removal rate of IFN$\beta$ from the body is lowered and the dosage interval is extended (188). GA is a synthetic peptide that mimics MBP and suppresses inflammatory responses via inhibition of encephalitogenic T cells and activation of Treg $(189,190)$. GA reduces the relapse rate by $29 \%$ compared to placebo in MS patients $(4,191)$. Both IFN$\beta$ and GA treatment have no long-term efficacy on progression of disability (3, $192,193)$. No long-term safety issues were assigned to these therapies. Common mild side effects are flu-like symptoms and injection site reactions $(3,194,195)$. However, new improved treatment options were desirable with a preference for developing oral treatments in order to exclude injection site pain. In 2012 and 2013, two new MS therapies, namely teriflunomide (Aubagio ${ }^{\mathrm{TM}}$ ) and DMF (Tecfidera ${ }^{T M}$ ), were licensed as the first two oral first-line therapies (3). Teriflunomide is a pyrimidine synthesis inhibitor that interrupts proliferation of $T$ and B cells (196). Teriflunomide reduces the annualized relapse rate by $31 \%$ compared to placebo and the most common side effects are gastrointestinal events and hair thinning (197, 198). DMF has neuroprotective and immunomodulatory effects $(199,200)$. Long-term safety and clinical efficacy of DMF, with a reduced annualized relapse rate of 53\% in RRMS patients compared to placebo, was demonstrated $(201,202)$. Compared to the injectable treatments, DMF has a beneficial impact on quality of life of RRMS patients because of its ease of use, lack of injection site pain and limited side effects (gastrointestinal events and flushing) $(202,203)$.

\subsubsection{Second- and third-line treatments}

When MS patients do not adhere to first-line treatments, second- and third-line treatments such as alemtuzumab (LemtradaTM), natalizumab (Tysabri ${ }^{\mathrm{TM}}$ ), fingolimod (Gilenya ${ }^{\mathrm{TM}}$ ), daclizumab (Zinbryta ${ }^{\mathrm{TM}}$ ), ocrelizumab (Ocrevus ${ }^{\mathrm{TM}}$ ) and cladribine (Mavenclad ${ }^{\mathrm{TM}}$ ) are used (181). The infusion therapy alemtuzumab is a humanized monoclonal antibody against CD52, which depletes circulating lymphocytes. Alemtuzumab has a high efficacy and reduces the relapse rate by $55 \%$ compared to IFN- $\beta$ but also has a high risk profile, since the most significant adverse event is the development of secondary autoimmune diseases and is therefore used as a therapy of last resort in the face of very active disease, meaning it is a second-line treatment at the moment (204-206). 
Natalizumab is a humanized monoclonal antibody against adhesion molecule $\mathrm{a}_{4}$ integrin expressed on lymphocytes and was the first second-line treatment approved in Europe in 2006. It inhibits immune cell trafficking across the BBB (3, 207). Natalizumab is highly efficient and decreases the annualized relapse rate by $69 \%$ in RRMS patients compared to placebo, however, patients are at risk of developing an opportunistic brain infection caused by the John Cunningham (JC) virus, called progressive multifocal leukoencephalopathy (PML), so a special prescription program is advised $(3,208,209)$. In 2010 , the first oral second-line drug, fingolimod, became available for MS patients. Fingolimod is a sphingosine1-phosphate receptor (S1PR) modulator which traps lymphocytes in the lymph nodes and most common side effects are bradyarrhythmia, increased risk of infections, liver dysfunction and lymphopenia. Because of its high efficacy, evidenced by a reduction in the relapse rate by $54 \%$ in RRMS patients compared to placebo, and long-term safety profile it is recommended for patients with highly active RRMS (4, 210-212). Daclizumab is a humanized anti-CD25 monoclonal antibody that was originally designed to inhibit activated T cells. However, it also induced an increase in the proliferation of regulatory NK cells which correlated with treatment response (213). Recently, the drug was withdrawn from the market due to liver toxicity, inflammatory encephalitis and meningoencephalitis (214). The B cell depletion therapies ocrelizumab (Ocrevus ${ }^{\mathrm{TM}}$ ) and cladribine (Mavenclad ${ }^{\mathrm{TM}}$ ) were approved in 2017 and 2018, respectively (181). Ocrelizumab is a humanized anti-CD20 monoclonal antibody that depletes B cells. Ocreluzimab was shown to reduce relapses by $46 \%$ compared to IFN- $\beta$ and reduces disability in RRMS as well as PPMS patients, and is thereby the first treatment approved for PPMS (215). Cladribine is an oral medication that interferes with DNA synthesis and repair of $T$ and $B$ cells resulting in selective and sustained depletion of $T$ and B cells. Cladribine reduces the relapse rate by $58 \%$ in RRMS patients compared to placebo and delays disability progression. Its common adverse event is lymphopenia. Cladribine is suitable for the treatment of rapidly evolving RRMS patients with highly active disease (216).

The most recent DMD Siponimod has been approved in January 2020 and is the first treatment for SPMS (217). Siponimod is a selective sphingosine 1-phosphate receptor 1 (S1PR1) and 5 (S1PR5) (218). Siponimod decreases egress of lymphocytes from lymphoid organs and prevents recirculation of peripheral 
lymphocytes to the CNS (219). In the phase III EXPAND study, siponimod reduced the risk of three-month confirmed disability progression by $21 \%$. In addition, Siponimod showed favorable outcomes in annualized relapse rate, MRI disease activity and brain volume loss compared to placebo (217).

\subsubsection{Off-label treatments for MS}

Treatments originally used in other diseases but frequently prescribed as off-label treatment for MS are rituximab (Rituxan ${ }^{T M}$ ) and mitoxantrone (Novantrone ${ }^{T M}$ ). Rituximab is a chimeric anti-CD20 B cell depleting monoclonal antibody.

It was the first therapeutic antibody approved for oncology patients and later in rheumatoid arthritis $(220,221)$. Since rituximab attenuates the inflammatory activity in MS patients and has clinical and radiological benefits, its widely used as an off-label therapy for MS (221). Mitoxantrone is another treatment for oncology patients which disrupts DNA synthesis and repair of cancer cells. However it also exerts immunosuppressive effects which is why it is sometimes prescribed for SPMS and RRMS patients who do not respond to first-line treatments $(3,222)$.

\subsubsection{Therapies in the pipeline}

Fingolimod has off-target effects which are attributed to its low receptor specificity, resulting in e.g. cardiac side effects. Therefore, several companies developed oral next generation S1PR modulators. Ozanimod is both specific for S1PR1 and S1PR5 while ponesimod is specific for S1PR1 only (218). Ozanimod reduces the annualized relapse rate by $38 \%$ compared to IFN- $\beta$ in RRMS patients and is a valuable contribution to the therapeutic armamentarium in MS since the once daily oral administration is convenient for MS patients (218). Ozanimod is approved by the FDA on March 252020 and approval by the EMA is pending (223). Considering ponesimod, two phase III trials are currently underway, for which results are expected in 2020. One compares oral ponesimod to teriflunomide and one compares ponesimod as an add-on to DMF to placebo (218). Two other oral therapies for RRMS are Evobrutinib (Bruton's Tyrosine Kinase (BTK) Inhibitor) and BIIB098 (diroximel fumarate) for which positive results are being gathered from phase IIb and phase III trials, respectively $(224,225)$. Diroximel fumarate has a similar working mechanism as DMF but is associated with fewer gastrointestinal side effects. New B depletion therapies, namely Ublituximab (targeting CD20 on $B$ cells) and Ofatumumab (first fully human anti-CD20 monoclonal antibody), have 
shown a favourable safety profile in phase II trials with RRMS patients while phase III trials are currently ongoing (226-229).

In order to reduce the frequency of injections, a GA long-acting injection (GA Depot) has been designed and the positive results in safety, tolerability and efficacy have prompted the continuation to a phase III trial (230). Other therapies that are expected to be on the market in the distant future are ATX-MS-146 (consists of epitopes from 4 regions of MBP) and ATL-1102 (small molecule which binds and blocks a mRNA sequence encoding the CD49d protein on the VLA-4 receptor) that showed promising results in phase IIa studies and PRN2246 (BTK inhibitor) that was successful in a phase I study (231-235). 


\subsection{DMF}

DMF is one of the newer additions to the armamentarium of potent immunomodulators for the treatment of RRMS. It has a strong efficacy with neuroprotective and immunomodulatory effects and a favourable benefit-risk profile. However, the effects of DMF on the immune system of MS patients were not clear before entering the market. During the last years, numerous in vitro and ex vivo studies have clarified the working mechanism of DMF. Here, we discuss the pharmacokinetics of DMF and its effect on molecular immune-related pathways. The efficacy and safety of DMF treatment for RRMS is discussed as reported from clinical trials.

\subsubsection{History of DMF as a therapeutic agent}

DMF is a fumaric acid ester, a small molecule with immunomodulating, antiinflammatory and anti-oxidative effects. Fumaric acids are intermediates of the citric acid cycle in humans, which is used by cells to produce energy. Fumaric acid esters have been used for years as a therapy for psoriasis, a chronic inflammatory skin disease mediated by skin-directed T cells resulting in scaly plaques (236238). In 1959 the chemist Schweckendiek, who suffered from psoriasis, hypothesized that psoriasis is caused by a disturbance in the citric acid cycle as a result of decreased levels of its component fumaric acid. As free fumaric acid has a gastrointestinal irritating effect and is poorly absorbed by the intestines, he developed a mixture of fumaric acid esters out of the Fumaria officinalis plant which cleared his psoriasis (239). Since 1994, a mixture of ethyl hydrogen

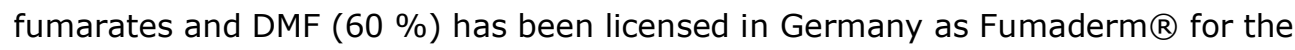
systemic treatment of severe psoriasis. Studies of Fumaderm $\AA$ reported high efficacy in psoriasis patients with an immunomodulatory potential and a positive long-term safety profile, which made it a potential candidate for the treatment of

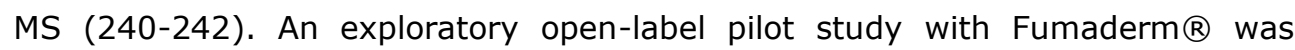
conducted in 6 RRMS patients already in 2006. This study reported an improvement of disease activity by reducing the brain lesion number and volume after 18 weeks of treatment (243). Furthermore, this pilot study revealed immunomodulatory effects of fumaric acids in RRMS patients, such as an increase in anti-inflammatory IL-10 expressing $\mathrm{CD}^{+} \mathrm{T}$ cells. Regardless of the small 
number of included patients, this first study suggested that clinical trials of fumaric acid esters in RRMS patients were warranted (243).

\subsubsection{Pharmacokinetics of DMF}

DMF is hydrolysed into its active metabolite monomethyl fumarate (MMF) by esterases in the small intestine. It was previously shown that an alkaline environment ( $\mathrm{pH} \mathrm{8),} \mathrm{as} \mathrm{is} \mathrm{present} \mathrm{in} \mathrm{the} \mathrm{small} \mathrm{intestine,} \mathrm{is} \mathrm{necessary} \mathrm{for} \mathrm{this}$ hydrolysis and that no hydrolysis of DMF occurs in an acidic environment $(\mathrm{pH} 1)$ resembling the stomach (244). Since only MMF and no DMF is detectable in the serum following DMF intake, it has long been thought that DMF is completely hydrolysed to MMF $(245,246)$. However, it has become clear that one part of DMF is hydrolysed into MMF while another reacts with glutathione (GSH) to form longlived GSH-conjugates that were detected in the urine of DMF-treated psoriasis patients (246-249). Thus, both MMF and DMF can exert biologically relevant activities. Furthermore, lymphocytes and monocytes were shown to effectively hydrolyse MMF and DMF, which resulted in a faster removal of MMF and DMF from whole blood than from serum (244). Plasma levels of MMF are highly variable, ranging from 1 to $5 \mu \mathrm{g} / \mathrm{ml}$ (equivalent to $10-40 \mu \mathrm{M}$ ), and are dependent on food intake $(245,250)$. Furthermore, DMF and MMF are physiologically active between 5.5 and $50 \mu \mathrm{M}$ (toxic at $150 \mu \mathrm{M}$ ) and 50 and $150 \mu \mathrm{M}$ (toxic at $450 \mu \mathrm{M}$ ), respectively (251). DMF and MMF exhibited a similar response profile in vitro but at distinctly different doses, namely $16.7 \mu \mathrm{M}$ for DMF and $150 \mu \mathrm{M}$ for MMF. Together, these findings could imply that different anatomic compartments and immune cell populations are exposed to different doses of DMF and MMF, which could have an influence on the working mechanism and therapeutic effects.

\subsubsection{Molecular mechanism of action of DMF}

\section{Nuclear factor erythroid-derived 2 (Nrf2) pathway activation}

The neuroprotective effect of DMF and MMF is caused by activation of the transcription factor nuclear factor erythroid-derived 2 (Nrf2) (200). Nrf2 is expressed as a redox sensor in every cell of the body, including neurons, astrocytes, microglia and immune cells (200, 252, 253). In vitro MMF treatment of human and rodent astrocytes led to a covalent modification of cysteine residue 151 of keap1 protein, which is the inhibitor or Nrf2 (Figure 1.4(a)) (200). This 
resulted in the dissociation of keap1 from Nrf2, Nrf2 activation and increased transcription of anti-oxidant target genes, including aldo-keto reductase family 1 member B10 (AKR1B10) and nicotinamide adenine dinucleotide phosphate $(\mathrm{NADP})(\mathrm{H})$ quinoline oxidoreductase-1 (NQO-1) (200). In this way, DMF/MMF could exert neuroprotective and cell survival effects $(200,254)$. Both DMF and MMF can covalently bind to cysteine residues of proteins via a process called protein succination. However, DMF and MMF have differing degrees of activity, which resulted in a greater accumulation of Nrf2 in human astrocytes treated in vitro with DMF than with MMF (255). In vivo, DMF treatment of mice with EAE, the animal model for MS, induced an increase in free Nrf2 in neuronal and glial cells, resulting in a beneficial effect on disease score (200). This effect was almost completely abolished when using Nrf2 knockout mice.

However, EAE studies using Nrf2 knockout mice also showed that not all immune modulating activity of DMF is dependent of the Nrf2 pathway. DMF treatment of both Nrf2 knockout and wild-type mice reduced EAE susceptibility $(256,257)$. Further, both Nrf2 knockout and wild-type EAE mice showed reduced frequencies of Th1 and Th17 cells and reduced B cell MHC-II expression (257). In addition, downregulation of the adhesion molecule VLA-4 was observed on T cells and $B$ cells of EAE mice following DMF treatment in a Nrf2-independent manner (256). Other molecular pathways involved in the neuroprotective and immune modulating effects of DMF are described below.

\section{Nuclear factor (NF)-KB pathway inhibition}

DMF, but not MMF, was shown to interact with cysteines in several proteins involved in the nuclear factor (NF)-KB pathway, such as IKB kinase $\beta$ (IKK $\beta$ ). Consistent with these targets, DMF blocked the nuclear translocation of $p 65$ and p52 in the NF-KB pathway (Figure $1.4(\mathrm{~b}))(251,258)$. One of the main targets of DMF was protein kinase C theta (PKC- $\theta$ ) (258), which is expressed in T cells where it is responsible for the engagement of the $T$ cell receptor (TCR) and the costimulatory molecule CD28. By blocking cysteine residues in PKC- $\theta$, DMF inhibited IL-2 secretion by activated T cells (Figure 1.4(c)). Consequently, DMF inhibited the production of NF-KB driven pro-inflammatory factors such as IL-6, IFN- $\mathrm{Y}$ induced protein 10 (IP10) and macrophage inflammatory protein (MIP)-1 $\beta$ by polyclonally stimulated human peripheral blood mononuclear cells (PBMC) 
(251). Correspondingly, another study indicated that DMF but not MMF inhibited the nuclear binding of NF-KB in purified human $\mathrm{T}$ cells, although the used concentration of MMF was probably too low (259).

\section{Hydroxycarboxylic acid receptor 2 (HCAR2) activation}

The contribution of the hydroxycarboxylic acid receptor 2 (HCAR2) to the clinical efficacy of DMF was shown in EAE mice where DMF treatment had positive effects on disease score, immune cell infiltration and demyelination in wild-type but not HCAR2 knockout mice (260). HCAR2 is a G protein-coupled receptor that is expressed by immune cells such as macrophages, microglia, neutrophils, epidermal Langerhans cells and dendritic cells, but not by lymphocytes (261-266). HCAR2 was indicated to exert an anti-inflammatory effect by inhibiting transcription of NF-KB related genes, which led to a switch from classically activated pro-inflammatory microglia to alternatively activated anti-inflammatory microglia in the CNS of EAE mice and the inhibition of pro-inflammatory molecule secretion (Figure 1.4(d)) (267). Further, HCAR2 activation was shown to induce apoptosis via decreasing cyclic adenosine monophosphate (AMP), to induce posttranslational modification of $\mathrm{B}$-cell lymphoma 2 ( $\mathrm{Bcl}-2)$ family members and to reduce monocyte/macrophage chemotaxis and adhesion to endothelial cells (262, $266,268,269)$. The interaction of MMF with HCAR2 was also detected in vivo, since HCAR2 could cause flushing, a side effect of DMF treatment in MS patients, via the release of prostaglandin $2(202,261)$.

\section{GSH depletion}

GSH is an anti-oxidant thiol that protects cells from oxidative stress, regulates cell proliferation, apoptosis and immune function (270). DMF can cause depletion of circulating GSH levels by binding to its free cysteines and forming GSH conjugates (Figure 1.4(e)) (271). Differential effects of DMF and MMF on GSH depletion from human astrocytes were observed, as DMF treatment transiently depleted intraand extracellular GSH levels during $10 \mathrm{~h}$ after which GSH levels gradually increased while MMF increased GSH levels after $24 \mathrm{~h}$ of treatment (255). GSH depletion could contribute to the immunosuppressive and anti-inflammatory properties of DMF as it leads to an induction of the anti-inflammatory stress protein heme oxygenase 1 (HO-1), which reduces pro-inflammatory cytokine secretion in activated human PBMC (272). The increase in total GSH after 
depletion may be a compensatory response or may be the result of GSH synthesis induced via the Nrf2 pathway by DMF and MMF (Figure 1.4(a)) (255, 270, 273). Additionally, DMF can induce GSH recycling via increasing glutathione reductase (GSR) which catalyses glutathione disulphide (GSSG) reduction to GSH by using NADPH (Figure 1.4(e)). The increase in GSH is important for the neuroprotective effect of DMF since it makes the cells resistant against oxidative stress $(255,273$, 274).

\section{Inhibition of aerobic glycolysis}

DMF and MMF were shown to interact with cysteine residues of the glycolytic enzyme glyceraldehyde 3-phosphate dehydrogenase (GAPDH), both in vitro and in vivo (Figure $1.4(\mathrm{f}))(275,276)$. Consequently, GAPDH is inactivated which results in an inhibition of the aerobic glycolysis in myeloid and lymphoid cells. Effector lymphocytes, such as Th1 and Th17 cells, require glycolysis for their proliferation, survival and effector functions such as cytokine production (277). On the contrary, differentiation of regulatory immune cells is driven by oxidative metabolism (276-278). Thus, DMF could mediate anti-inflammatory effects in lymphocytes by downregulating the glycolysis (276). 


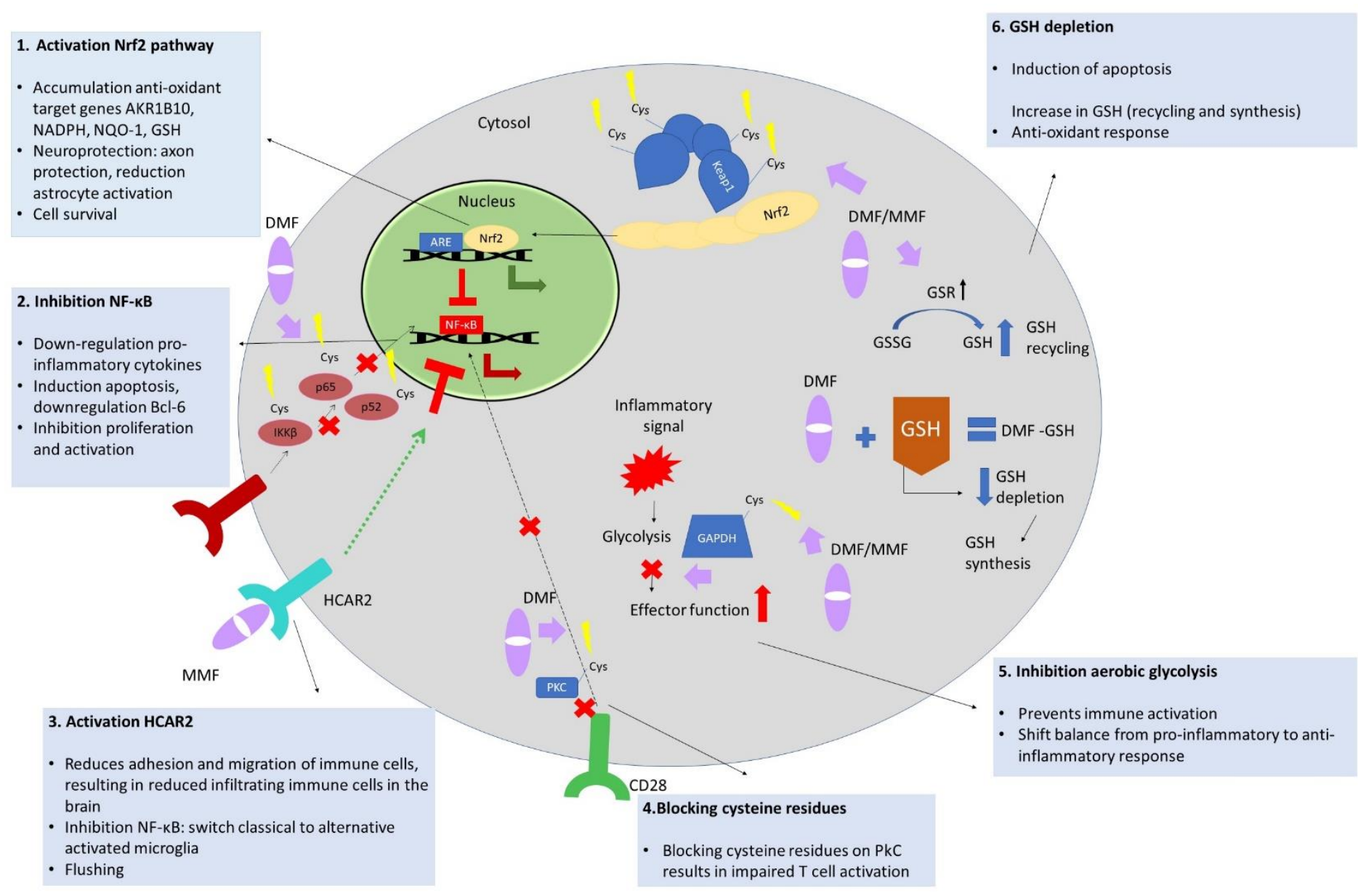


Figure 1.4 Molecular pathways of DMF treatment. (a) DMF and MMF react with cysteine residues of Keap1, which causes Keap1 to dissociate from Nrf2 leading to the nuclear translocation of Nrf2. Nrf2 then binds to the antioxidant response element (ARE) and drives the expression of anti-oxidant target genes, which leads to neuroprotection, reduction of astrocyte activation and cell survival. (b) DMF modifies cysteine residues of proteins involved in the NF-KB pathway (IKK $\beta, p 65, p 52$ ), which leads to the inhibition of p65 and p52 translocation. Inhibition of NF-KB then leads to inhibition of pro-inflammatory cytokine production and inhibition of cell activation and proliferation. (c) In T cells, DMF modifies cysteine residues of PKC- $\theta$ resulting in impaired activation of $T$ cells and interruption of NF-KB activation. (d) MMF is a potent agonist of HCAR2, which results in reduced migration and infiltration of immune cells in the CNS. HCAR2 inhibits the NF-KB pathway, which results in a switch of classically activated pro-inflammatory microglia to alternatively activated microglia and the inhibition of pro-inflammatory molecule production. The binding of MMF to HCAR2 also results in the induction of apoptosis and causes flushing in MS patients. (e) DMF induces a transient GSH depletion by interacting with cysteine residues of GSH and subsequently forming DMF-GSH conjugates, which leads to apoptosis induction, immunosuppressive and anti-inflammatory effects. GSH depletion induces GSH synthesis directly, but DMF and MMF also induce GSH synthesis indirectly via GSH recycling by increasing the enzyme GSR which converts GSSG into GSH. An increase in GSH exerts a neuroprotective effect by inducing an anti-oxidant response. (f) DMF and MMF inhibit aerobic glycolysis in immune cells by modifying cysteine residues of GAPDH, which results in inhibition of immune cell activation and a shift from a pro-inflammatory to an antiinflammatory response. DMF = dimethyl fumarate, MMF = monomethyl fumarate, Keap1 = Kelch-like ECH-associated protein 1, Nrf2 = nuclear factor (erythroid-derived 2)-like 2, ARE $=$ antioxidant response element, $\mathrm{AKR} 1 \mathrm{~B} 10=$ aldo-keto reductase family 1 member B10, $\mathrm{NADPH}=$ nicotinamide adenine dinucleotide phosphate, $\mathrm{NQO}-1=\mathrm{NAD}(\mathrm{P}) \mathrm{H}$ dehydrogenase (quinone 1), GSH = glutathione, IKK $\beta=\mathrm{I} \kappa B$ kinase $\beta, \mathrm{NF}-\mathrm{KB}=$ nuclear factor $-\kappa \mathrm{B}, \mathrm{HCAR2}$ $=$ hydroxycarboxylic acid receptor $2, \mathrm{PKC}-\theta=$ protein kinase $\mathrm{C}$-theta, GAPDH = glyceraldehyde-3-phosphate dehydrogenase, GSR = glutathione reductase, GSSG = glutathione disulfide. 


\subsubsection{Efficacy of DMF in animal models for MS}

Based on the results and the clinical success of the 2006 pilot study in RRMS patients (243), Schilling et al. investigated the efficacy of DMF in EAE (279). Prophylactic administration of DMF or MMF resulted in a decreased disease activity and reduced spinal cord infiltration of $\mathrm{T}$ cells and macrophages compared to control animals receiving vehicle alone (279). Later, Linker et al. reported a reduced disease activity following both prophylactic and therapeutic DMF treatment in the chronic phase of EAE (200). DMF exerted neuroprotective effects reflected by a $60 \%$ reduction of demyelination, by neuronal and myelin preservation and by a reduced activation of astrocytes (200). In a spontaneous chronic EAE model in which both $T$ and $B$ cell receptors were specific for MOG, low dose DMF treatment resulted in a decreased disease activity, less demyelination and less macrophage infiltration in the spinal cord compared to control mice treated with vehicle alone and to mice treated with a high dose of DMF (280). Schulze-Topphoff et al. confirmed a reduced severity and incidence of spontaneous chronic EAE after DMF treatment (257). Furthermore, in an acute EAE model, combination treatment of DMF and IFN- $\beta$ ameliorated the EAE disease course (281). Hereby, DMF-treated mice showed higher axonal densities and milder demyelination compared to control mice receiving vehicle and compared to mice receiving IFN- $\beta$ monotherapy.

\subsubsection{Clinical trials of DMF treatment in RRMS patients}

\section{Efficacy}

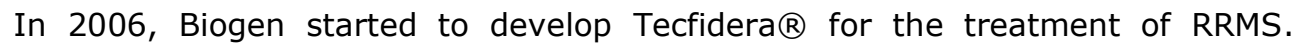

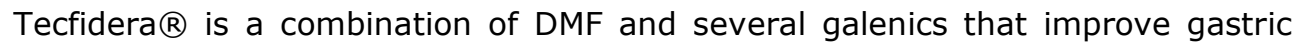
tolerability of the drug when compared to previous formulations (282). The efficacy of DMF in enteric-coated capsules in the treatment of RRMS in adults was evaluated in 2008 in a randomized, multi-center, double blind, placebo controlled phase II trial (283). Patients were randomized to receive either placebo, $120 \mathrm{mg}$ DMF once daily, $120 \mathrm{mg}$ DMF three times daily or $240 \mathrm{mg}$ DMF three times daily for 24 weeks. The mean total number of new gadolinium enhancing (GdE) lesions on MRI (primary endpoint) was reduced by 69\% from week 12 to 24 in the 240 $\mathrm{mg}$ three times daily treated RRMS patients (283). 
Subsequently, two phase III trials were conducted, namely Determination of the Efficacy and Safety of Oral Fumarate in RRMS (DEFINE) and Comparator and an Oral Fumarate In RRMS (CONFIRM) (201, 202). The DEFINE study was a double blind study where patients were randomly assigned to receive either placebo, DMF at $240 \mathrm{mg}$ twice daily or three times daily. The proportion of RRMS patients who had a relapse within 2 years of DMF treatment (primary endpoint, $27 \%$ for DMF twice daily and $26 \%$ for DMF three times daily) reduced significantly compared to placebo (46\%) (202). In the CONFIRM study, patients were randomly assigned to receive placebo, DMF twice or three times daily and patients taking GA were used as a comparator. After 2 years of treatment, the annualized relapse rate (primary endpoint) was significantly reduced in the two DMF-treated groups (0.22 for DMF twice daily and 0.20 for DMF three times daily) and the GA treated group (0.29) compared to placebo (0.40) (201). As a result, DMF was approved by the FDA and the EMA to be prescribed as a first-line therapy for RRMS patients at a dosing of $240 \mathrm{mg}$ twice a day. Integrated analyses of the two phase III studies showed a high efficacy of DMF in newly diagnosed RRMS patients with a low EDSS score (284).

Disability was measured using the Multiple Sclerosis Functional Composite (MSFC) in the DEFINE and CONFIRM studies (285). The MSFC assesses motor function of the leg and ambulation with the Timed 25-Foot Walk (T25W), arm and hand function with the 9-Hole Peg Test (9HPT), and cognitive functions, namely auditory processing speed and attention with Paced Auditory Serial Addition Test (PASAT-3). MS patients treated with DMF showed an improved and superior MSFC compared to placebo (285).

\section{Safety}

The DEFINE and CONFIRM studies showed mild to moderate side effects of DMF including gastro-intestinal events and flushing which decreased after 1-2 months $(201,202)$. To increase tolerability, patients are recommended to start with 120 $\mathrm{mg}$ for a week after which the dose should be increased up to $240 \mathrm{mg}$ twice a day. The patients of the DEFINE and CONFIRM studies were followed for another 3 years in a long-term extension study (ENDORSE), which resulted in a total followup of 5 years (286). The safety profile of DMF was acceptable and comparable with the DEFINE/CONFIRM studies. However, a case of PML was reported in a 
DMF-treated MS patient with severe prolonged lymphopenia (287). In 2016, already four other cases of PML were reported (287). Nonetheless, in RRMS patients continuing DMF treatment, mean white blood cells and lymphocyte counts remained stable throughout time (286). To reduce the risk of $\mathrm{PML}$, it is recommended to monitor lymphocyte counts before treatment and every 3 months (EMA) or every 6-12 months (FDA) thereafter. Discontinuation of DMF should be considered for MS patients experiencing lymphopenia (< 500 cells/mm3) persisting for more than 6 months (286).

In conclusion, clinical trials of DMF have shown strong efficacy and a sustained and favourable benefit-risk profile for RRMS patients. Detailed analysis of clinical parameters indicated that DMF treatment protects MS patients from a progressive decline in motor and cognitive function. 


\subsection{Aims of the study}

The immune system of MS patients is characterized by a dysregulation in the balance between pro- and anti-inflammatory immune cells, whereby both the innate and adaptive immune system are involved. In the last decades, numerous disease-modifying treatments have emerged for MS. Despite the fact that many treatments are designed to target a specific pathological pathway or immune cell type in MS, the working mechanism of these treatments is often multifactorial and not fully unravelled when they enter the market. Since MS patients and physicians have to choose between numerous treatment options, it is important to investigate the working mechanism of these treatments. This in turn leads to improved insight into the role of the different immune cell subtypes in MS pathology. Furthermore, the positive results observed in MS patients treated with $B$ cell depleting therapies have emphasized that the role of B cells in the pathology of MS should be evaluated in more detail. I aim to study the contribution of immune cell populations to MS pathology, on the one hand by analysing the effect of DMF treatment on the distribution and function of immune cell populations and, on the other hand, by studying the phenotype and function of age-associated $B$ cells in MS patients.

\section{Aim1: Performing an extensive immunophenotypic analysis of the innate and adaptive immune system of RRMS patients under DMF treatment}

In 2013, a new MS therapy, DMF, was licensed as the first oral therapy that could be prescribed as a first-line therapy. It has a strong efficacy with neuroprotective and immunomodulatory effects and a favourable benefit-risk profile. However, the effects of DMF on the immune system of MS patients were not clear before entering the market.

We aim to explore whether DMF contributes to the restoration of the immune homeostasis in MS patients. Based on previous studies performed ex vivo on psoriasis patients, in vitro on $\mathrm{HC}$ and in $\mathrm{EAE}$, we hypothesize that DMF therapy shifts the humoral and cellular immune response of MS patients from a proinflammatory to an anti-inflammatory profile. Determining the mechanism of action of DMF is crucial in order to understand and subsequently prevent its possible side effects. This information may also prove important for the improvement of the existing MS therapies, as well as for the development of new 
treatment options. Finally, understanding the mechanism of action of DMF on the immune system of MS patients will give us more insight in the role of these immune cells in the pathogenesis of MS.

No information is available on the effects of DMF on the distribution of $\mathrm{T}$ and $\mathrm{B}$ cell subtypes in MS. As DMF promoted an anti-inflammatory cytokine profile in vitro (288), we hypothesize that pathogenic immune cells are decreased, while regulatory $T$ and $B$ cells are increased following DMF treatment of MS patients. In Chapter 2, T cell subtypes, B cell subtypes, innate immune cell subtypes, T cell cytokines and the expression of costimulatory, antigen presentation and survival markers on B cells are analyzed during a 12-month follow-up study in 21 RRMS patients at 3 time points: before initiation of treatment with DMF, after 12 weeks of DMF treatment and after 48 weeks of DMF treatment. Results obtained before the initiation of DMF treatment are used as control. Findings from this part of the study demonstrate whether DMF restores the immune balance in MS patients by decreasing the pathogenic $B$ and $T$ cell subtypes and increasing the regulatory $B$ and $T$ cell subtypes. Furthermore, in order to identify at which time point DMF is effective and to determine the long-term effect of DMF, the effect of DMF on T cell subtypes is investigated in a cross-sectional study in 64 DMF treated MS patients at different time points and 25 untreated MS patients as a control group. Findings from this part of the study will show at which time points DMF treatment is fully effective and if the effect of DMF on the distribution of immune cells will be stable after a year of treatment.

\section{Aim 2: Assess the direct effects of DMF and MMF on the function of B cells in MS patients}

The positive results of B cell depleting treatment in MS patients emphasize that it is important to investigate the effect of current treatments on subtypes and functions of B cells. B cells have become increasingly recognized as key players in MS pathogenesis, both by production of (auto)antibodies and by antibodyindependent functions, including the stimulation of pro-inflammatory $\mathrm{T}$ cell responses via cytokine production, costimulation and antigen presentation.

It was already shown that DMF can directly induce apoptosis of T cells and reduce the pro-inflammatory cytokine producing $T$ cells, however, no information was 
available on the direct effect of DMF on B cells. Since it is well described in in vitro studies that DMF has immunomodulating effects and interferes in the function of different immune cells, it is important to figure out what the direct effects of DMF and MMF are on B cells. In Chapter 3, the direct effects of DMF and MMF on B cell apoptosis and expression of costimulatory molecules (CD40, CD80, CD86), antigen presentation molecules and the survival marker BAFFR is evaluated in HC and untreated MS patients. Findings from this part of the study will show if DMF can directly induce apoptosis in B cells and if it can interfere with the antigen presentation and costimulation function of $B$ cells.

\section{Aim 3: Identify the phenotype of age-associated B cells and their migration and activation capacity in MS}

Due to the emergence of $B$ cell depleting treatment there is a growing body of evidence emphasizing the importance of $B$ cells in the pathogenesis of MS. Premature aging of the immune system has been evidenced in a proportion of MS patients. Age-associated B cells as a highly promising candidate pathogenic B cell subtype in autoimmune disease. Age-associated B cells have also been described in humans during healthy aging and in autoimmune conditions. Novel findings in mouse models put forward T-bet ${ }^{+}$. Recent literature and our previous findings demonstrate the possible involvement of age-associated $B$ cells in pathological processes of MS and underline the importance of studying their functional properties. We aim to study the phenotype of expanded DN B cell populations in MS and further study the activation and migration capacity of DN in MS. We thereby hypothesize that T-bet expression is higher in age-associated $B$ cells (DN and $C D 21^{\text {low }} B$ cells) compared to naive and memory $B$ cells and that DN B cells have a higher migration and activation capacity. Since details about the function of age-associated B cells in pathological processes of MS remain to be elucidated, further investigation of these cells contributes to a better understanding of MS pathogenesis. Moreover, it could have added value in discovering new therapeutic targets that can also be beneficial for other autoimmune diseases in which ageassociated B cells are implicated, such as SLE and RA.

In humans, it is demonstrated that DN B cells B cells with pro-inflammatory characteristics are abnormally elevated in a proportion of MS patients (68). In Chapter 4, a detailed phenotyping of DN B cells from MS patients is performed 
using developmental markers and markers for adhesion and migration. Secondly, the expression of T-bet in age-associated $B$ cells (DN and CD21 low $B$ cells) is investigated and compared to the expression of T-bet in memory and naive B cells to further unravel the pathway driving the development of age-associated $B$ cells. Thirdly, functional characteristics of DN B cells of MS patients and HC such as migration and activation status is evaluated and compared to memory and naive $B$ cells. Findings from this part of the study will unravel the phenotype of DN $B$ cells and will show if DN B cells have a higher capacity to migrate to inflammatory sites and become activated compared to naive and memory $B$ cells. 


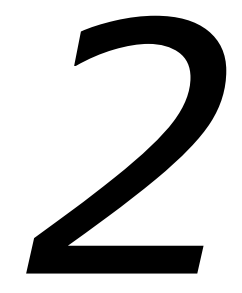

Dimethyl fumarate induces a persistent change in the composition of the innate and adaptive immune system in multiple sclerosis patients

Based on:

Dimethyl fumarate induces a persistent change in the composition of the innate and adaptive immune system in multiple sclerosis patients Montes Diaz G., Fraussen J., Van Wijmeersch B., Hupperts R. and Somers V. Sci Rep. 2018 May 29;8(1):8194. 


\section{Dimethyl fumarate induces a persistent change in the composition of the innate and adaptive immune system in multiple sclerosis patients}

\subsection{Abstract}

The effects of dimethyl fumarate (DMF) on the immune system in multiple sclerosis (MS) are not completely elucidated. In this study, an extensive immunophenotypic analysis of innate and adaptive immune cells of DMF treated MS patients was performed. Peripheral blood immune cell phenotypes were determined using flow cytometry in a follow-up study of 21 MS patients before, after 3 and 12 months of DMF treatment and a cross-sectional study of 25 untreated and 64 DMF-treated MS patients. After 12 months of DMF treatment, percentages of monocytes, regulatory natural killer cells, naive $T$ and $B$ cells and transitional $B$ cells increased. Percentages of (effector) memory $T$ cells, (non) class-switched memory B cells and double negative B cells decreased together with $\mathrm{CD}^{+} \mathrm{T}$ cells expressing interferon- $\gamma$ (IFN- $\gamma$ ) and granulocyte macrophage colony-stimulating factor (GM-CSF). DMF treatment was fully effective as of 6 months. DMF induced a persistent change of the immune system of MS patients. 


\subsection{Introduction}

MS is a chronic inflammatory disease of the CNS in which both the innate and adaptive immune system are involved. The immune system of MS patients displays a disrupted balance favoring pro-inflammatory responses. T cells have long been considered as the most important players although the emergence of $B$ cell depleting treatment has emphasized the importance of $B$ cells in MS pathogenesis $(59,63)$. Adaptive immune cell subtypes involved in the pathological processes during MS are memory T cells (CD45RO $\left.{ }^{+} C D 45 R A^{-}\right)$and more specifically C-C chemokine receptor type 7 (CCR7)- effector memory T cells, CD8 ${ }^{+} \mathrm{T}$ cells and memory $\left(\mathrm{CD} 27^{+}\right) \mathrm{B}$ cells $(71,86,123,289-291) . \mathrm{CD}^{+} \mathrm{T}$ helper (Th) 1 and Th17 cells produce pro-inflammatory cytokines including interferon-y (IFN- $\mathrm{Y}$ ), interleukin-17 (IL-17) and granulocyte macrophage colony-stimulating factor (GM-CSF) while Th2 and regulatory T (Treg) cells produce anti-inflammatory IL-4 and IL-10 (65, 71). Double negative (DN, IgD-CD27-) B cells with a proinflammatory cytokine profile are described to be increased in a proportion of MS patients (68). For the innate immune system, monocytes $\left(C D 14^{+}\right)$, neutrophils and natural killer (NK) cells, including CD56 dim and CD56 bright NK cells, are involved in MS pathogenesis $(79,292-294)$.

Dimethyl fumarate (DMF, Tecfidera ${ }^{\circledR}$ ) was licensed as an oral first-line treatment for MS in 2013. Two phase III clinical trials, DEFINE and CONFIRM, demonstrated clinical efficacy of DMF in relapsing-remitting (RR) MS (201, 202). DMF reduced the annualized relapse rate and reduced the mean number of new or enlarging MRI lesions throughout the course of the study $(201,202)$. Earlier studies have demonstrated that DMF and its active metabolite monomethyl fumarate (MMF) exert neuroprotective and immunomodulatory effects through the activation of transcription factor nuclear factor (erythroid-derived 2)-like 2 (Nrf2) and via the suppression of transcription factor nuclear factor kappa b (NF-kB) $(200,259)$.

A number of studies investigated the effect of DMF on certain subtypes of diseaserelated immune cells $(199,295)$. Nevertheless, the working mechanism of DMF is multifactorial and the pathogenesis of $M S$ is directed by interplay between different immune cell subtypes. 
Previous studies were hampered by several conceptual limitations. The use of a cross-sectional approach prevented several studies to determine the cause-andeffect relationship at the individual level. In longitudinal studies, a limited followup of 3 to 6 months was insufficient to demonstrate a persistent change. The inclusion of patients previously treated with second-line therapies, such as fingolimod or natalizumab, could have influenced the lymphocyte numbers. In addition some studies examined the effect of DMF on solely one cell type. For these reasons we performed an extensive immunophenotypic analysis of the innate and adaptive immune system of RRMS patients under DMF treatment using 2 study designs: a 12 month $(\mathrm{m})$ follow-up study and a cross-sectional study. In this way, we further explored changes of several immune cell types over a longer period in a cause-and-effect way in untreated MS patients or in MS patients previously treated with first-line treatments. 


\subsection{Materials and methods}

\subsubsection{Study subjects}

RRMS patients and healthy controls ( $\mathrm{HC}$ ) were recruited at the Zuyderland Medical Center (Sittard, The Netherlands), Rehabilitation \& MS-Center (Overpelt, Belgium) and Biomedical Research Institute (Diepenbeek, Belgium). This study was approved by the institutes' ethics committees (METC14-T-96, $15.143 /$ neuro15.12) and all study subjects gave written informed consent. For the follow-up study, peripheral blood was collected before, after $3 \mathrm{~m}$ and $12 \mathrm{~m}$ of DMF treatment. Clinical outcome measures including the Expanded Disability Status Scale (EDSS) and the Fatigue Scale for Motor and Cognitive Functions (FSMC), a 20-item questionnaire for motor and cognitive functions were analyzed. Furthermore, the timed 25-foot walk (T25FW), the Paced Auditory Serial Addition Test (PASAT- $3^{\prime \prime}$ ), an auditory test of processing speed and attention and the Nine Hole Peg test (NHPT) were analyzed according to the Multiple Sclerosis Functional Composite (MSFC). All samples were stored in the University Biobank Limburg. Further clinical details and numbers of MS patients and $\mathrm{HC}$ are provided in Table 2.1. In all analyses, $\mathrm{HC}$ were matched to MS patients as closely as possible with regard to age and sex. 


\section{Table 2.1 Characteristics of study subjects}

${ }^{1}$ mean age in years; ${ }^{2}$ number of female (F) and male (M) individuals; ${ }^{3}$ mean Expanded Disability Status Scale (EDSS). IFN = interferon $-\beta$, Fingo = fingolimod, RRMS = relapsingremitting multiple sclerosis, DMF = dimethyl fumarate, $\mathrm{HC}=$ healthy control, $\mathrm{m}=$ months, N.A. = not applicable, UT $=$ untreated, $T F=$ teriflunomide, GA: glatiramer acetate
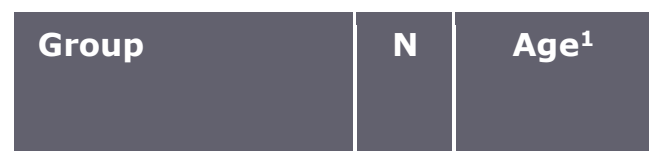

Female
$\%(F: M)^{2}$

EDSs $^{3}$

Previous treatmen $\mathbf{t}$

\section{Follow-up study}

\section{Included}

RRMS DMF

treated

$$
27 \quad 38 \pm 12 \quad 70(19 / 8) \quad 2.5 \pm 1.1
$$

UT: 17, IFN: 7, GA: 2, Fingo: 1

\section{Finished duration}

\section{of the study}

RRMS DMF

treated

$$
21 \quad 37 \pm 12 \quad 66(14 / 7) \quad 2.5 \pm 1.2 \quad \text { UT: } 15, \text { IFN : } 6
$$

\begin{tabular}{|c|c|c|c|c|c|}
\hline $\begin{array}{l}\text { Cross-sectional } \\
\text { study } \\
\text { HC }\end{array}$ & 10 & $30 \pm 6$ & $60(6 / 4)$ & N.A. & N.A. \\
\hline RRMS UT & 25 & $36 \pm 11$ & $72(18 / 7)$ & $2.5 \pm 1$ & $\begin{array}{l}\text { UT: } 19, \text { IFN: } 4 \text {, } \\
\text { GA: } 2\end{array}$ \\
\hline $\begin{array}{l}\text { RRMS DMF } \\
\text { treated 0-5 m }\end{array}$ & 23 & $37 \pm 9$ & $61(14 / 9)$ & $2.1 \pm 1.2$ & $\begin{array}{l}\text { UT: } 15, \text { IFN: } 5 \text {, } \\
\text { TF: } 3\end{array}$ \\
\hline $\begin{array}{l}\text { RRMS DMF } \\
\text { treated 6-12 m }\end{array}$ & 23 & $36 \pm 10$ & $70(16 / 7)$ & $1.7 \pm 1.2$ & $\begin{array}{l}\text { UT: } 10, \text { IFN: } \\
\text { 10, GA: } 1, \text { TF: } \\
1\end{array}$ \\
\hline RRMS $>12 \mathrm{~m}$ & 18 & $35 \pm 10$ & $72(13 / 5)$ & $1.6 \pm 1.4$ & $\begin{array}{l}\text { UT: } 3, \text { IFN: } 12 \text {, } \\
\text { GA: } 2, \text { TF: } 1\end{array}$ \\
\hline
\end{tabular}

\subsubsection{Cell isolation}

Peripheral blood mononuclear cells (PBMC) were isolated from whole blood by Ficoll density gradient centrifugation (Lympholyte, Cedarlane Laboratories, Uden, The Netherlands). PBMC of MS patients and HC were cryopreserved and thawed before use, unless stated otherwise. PBMC collected at different time points during the follow-up study were simultaneously thawed to exclude inter-assay variation. PBMC were cryopreserved in 10\% dimethyl sulfoxide (DMSO, Sigma Aldrich, 
Overijse, Belgium) and fetal bovine serum (FBS, Life Technologies, Gent, Belgium) using a slow temperature-lowering method (Coolcell ${ }$, VWR, Haasrode, Belgium).

After $24 \mathrm{~h}$, cryovials were transferred to liquid $\mathrm{N}_{2}$ until analysis. PBMC were thawed by bringing the temperature of the cryovials to $0^{\circ} \mathrm{C}$ in a water bath $\left(37^{\circ} \mathrm{C}\right)$. Hereafter, cold thawing medium consisting of 20\% FBS in RPMI 1640 (Lonza, Basel, Switzerland) was added and PBMC were centrifuged at $4^{\circ} \mathrm{C}$. PBMC were suspended in thawing medium in a concentration of $10 \times 10^{6}$ cells $/ 1000 \mu \mathrm{l}$ containing DNAse $(1 / 100)$ and incubated at $37^{\circ} \mathrm{C}$ for 10 minutes. Subsequently, cells were washed twice. Median cell recovery was $76 \pm 18 \%$.

\subsubsection{Flow cytometry}

In the follow-up study, PBMC were stained with anti-human CD14 PerCP, CD16 FITC, CD56 PE-Cy7 (BD Biosciences, Erembodegem, Belgium) and CD3 APC-Cy7 (BioLegend, Antwerp, Belgium). B cell subtypes were analyzed using anti-human CD19 PE-Cy7, immunoglobulin (Ig)M PerCP-Cy5.5 (BioLegend), CD27 APC, IgD PE-CF594, IgG FITC (BD Biosciences), CD38 APC-ef780 (eBioscience, San Diego, USA) and IgA PE (Miltenyi Biotec, Leiden, The Netherlands). T cell subtypes were analyzed with anti-human CD4 APC (BioLegend), CD45RA APC-ef780, CD127 PE, CD25 PE-Cy7 (eBioscience), CD45RO PE-CF594, CD8 FITC and CCR7 PerCP-Cy5.5 (BD Biosciences). Expression of survival markers was measured using anti-human CD19 PE-Cy7, CD268 APC-Cy7, CD267 APC, CD269 PE (Biolegend) CD27 FITC and IgD PE-CF594 (BD Biosciences). Expression of costimulatory and antigen presentation molecules was assessed using anti-human CD19 PerCP-Cy5.5, CD27 APC, CD80 PE, CD86 PE-CF594, HLA-DR/DP/DQ FITC (BD Biosciences) and CD40 $\mathrm{PE}-\mathrm{Cy} 7$ (eBioscience).

For intracellular T cell cytokine analysis, PBMC were stimulated with phorbol 12myristate 13-acetate (PMA, $50 \mathrm{ng} / \mathrm{ml})$ and calcium ionomycin $(1 \mu \mathrm{g} / \mathrm{ml}$, SigmaAldrich, Overijse Belgium) in the presence of Golgistop (BD Biosciences) for $4 \mathrm{~h}$. PBMC were fixed and permeabilized using Cytofix/Cytoperm (BD Biosciences) and stained with anti-human IL-10 PE-Cy7, GM-CSF APC, CD4 PE-Cy5 (BioLegend), IL-4 PE, IL-17 FITC (eBioscience) and IFN-y PE-CF594 (BD Biosciences). Freshly isolated PBMC were analyzed in the cross-sectional study using anti-human CD4 $A P C, C D 8 P E, C D 45 R O$ (BD Biosciences). All flow cytometry was performed on a 
FACSAria II or LSRFortessa flow cytometer using FACSDiva software (BD Biosciences).

Absolute numbers of leukocytes, lymphocytes, monocytes and neutrophils were obtained from standard clinical lab tests for 12 DMF-treated MS patients. Absolute numbers of lymphocyte populations were calculated as followed: absolute lymphocyte number $\left(10^{9} / \mathrm{L}\right) \times$ percentages of lymphocyte cell populations $\times 10$.

\subsubsection{ELISA}

BAFF plasma levels were quantified before and after $12 \mathrm{~m}$ of DMF treatment using a commercial ELISA kit according to the manufacturer's protocol (R\&D systems, Abingdon, United Kingdom).

\subsubsection{Statistics}

Statistical analyses were performed using Graphpad Prism 6. Wilcoxon matchedpairs signed rank test was used for comparison of variables at baseline and followup. When comparing multiple groups, one-way ANOVA (Kruskal-Wallis) was used. A p value less than 0.05 was considered statistically significant. 


\subsection{Results}

\subsubsection{Clinical characteristics}

In the follow-up study, 27 RRMS patients were enrolled and peripheral blood was collected before treatment, after $3 \mathrm{~m}$ and after $12 \mathrm{~m}$ of DMF treatment. Twentyone of the 27 MS patients finished the total duration of the study, while 6 MS patients dropped out of the study, with 2 due to side effects (gastrointestinal and flushing), 1 due to pregnancy, 1 due to other medication use, 1 due to lymphopenia and 1 due to an allergic reaction to DMF. Eighteen of the 21 DMF treated MS patients underwent MRI before and after at least $6 \mathrm{~m}$ of DMF treatment. In 14 MS patients, no new or enlarged lesions were detected. Furthermore, 4 of these 18 MS patients showed lesions that were decreased in volume or were less pronounced compared to baseline.

The average EDSS of 21 DMF-treated MS patients remained stable from baseline (2.4) until $12 \mathrm{~m}$ of DMF treatment (2.3). However, when considering individual MS patients, EDSS improved for 8 patients, remained stable for 7 patients, and increased for 6 patients. The MS patients with an increased EDSS were not excluded because of a clinical treatment response, a stable MRI and no relapse during the study period. Clinical outcome measures were determined for 14 DMFtreated MS patients (Table 2.2). Interestingly, a significantly improved cognitive function, namely auditory processing speed and attention measured by the PASAT, was observed after $12 \mathrm{~m}$ of DMF treatment (49.8 \pm 4.5 vs $54.2 \pm 3.7$, p $=0.0195$ ). The MSFC test remained stable in the NHPT (dominant hand: $20.2 \mathrm{~s}$ \pm 4.1 vs $19.3 \mathrm{~s} \pm 2.7$; non-dominant hand: $20.1 \mathrm{~s} \pm 3.3 \mathrm{vs} 19.7 \mathrm{~s} \pm 3$ ) and in the T25FW (4.7 s \pm 1.8 vs $5.3 \pm 2.2$ ) test over the course of the study. Furthermore, patient reported motor fatigue and cognitive fatigue as measured using the FSMC (a 20-item questionnaire for motor and cognitive functions), remained stable $(31.8 \pm 6.3$ vs $32.4 \pm 6.6$ and $34.2 \pm 9.7$ vs $33.4 \pm 9.6$, respectively) over the course of the study. 


\subsubsection{Reduced absolute numbers of lymphocytes, total $T$ cells and $B$ cells after DMF treatment}

Absolute immune cell numbers of leukocytes, lymphocytes, monocytes and neutrophils were obtained from standard clinical lab tests for 12 DMF-treated MS patients. Absolute leukocyte numbers decreased non-significantly (11\%) while lymphocyte numbers significantly decreased (46\%) after $12 \mathrm{~m}$ of DMF treatment ( $p=0.0024$, Table 2.2). Grade 3 lymphopenia (absolute lymphocyte numbers < $0.5 \times 10^{9}$ cells/L) was not observed in this patient cohort. Absolute monocyte and neutrophil numbers remained stable. Specific cell subtypes of both the innate and adaptive immune system showed significantly reduced numbers after $12 \mathrm{~m}$ and some already after $3 \mathrm{~m}$ of DMF treatment. The absolute number of CD56 dim NK cells was significantly reduced $(p=0.0034)$, while CD56 bright $N K$ cell number remained stable after $12 \mathrm{~m}$ of DMF treatment $(p=0.5186)$. The absolute numbers of $T$ cells $\left(\mathrm{CD}^{+}\right)$and all T cell subtypes were significantly decreased $(p<0.0161)$ after $12 \mathrm{~m}$ of DMF treatment, except for naive CD4 ${ }^{+} \mathrm{T}$ cells $(\mathrm{p}=0.3013)$. The absolute numbers of CD8 ${ }^{+} T$ cells $(p=0.0391)$ and memory CD4 ${ }^{+} T$ cells $(p=$ 0.0391) already decreased after $3 \mathrm{~m}$ of DMF treatment. Within the $\mathrm{B}$ cell population, the absolute numbers of total B cells (CD19+) and all B cell subtypes were decreased $(p<0.05)$ after $3 \mathrm{~m}$ and $12 \mathrm{~m}$ of DMF treatment, except for transitional $B$ cells $\left(C D 19^{+} \mathrm{CD} 27^{-} \mathrm{CD} 38^{+}\right)$, which were not affected $(p>0.999)$. In conclusion, most immune cell subtypes showed decreased circulating absolute numbers following $12 \mathrm{~m}$ of DMF treatment. Therefore, it was important to investigate the remaining peripheral blood immune cell population by determining immune cell subtype percentages. 
Table 2.2 Clinical tests and absolute numbers of immune cells after DMF treatment

\begin{tabular}{|c|c|c|c|}
\hline Clinical test & $\begin{array}{l}\text { Baseline } \\
\text { mean } \pm \text { SD }\end{array}$ & $\begin{array}{l}3 \text { m DMF } \\
\text { mean } \pm \text { SD }\end{array}$ & $\begin{array}{l}12 \mathrm{~m} \text { DMF } \\
\text { mean } \pm \text { SD }\end{array}$ \\
\hline EDSS & $2.4 \pm 1.2$ & $2.5 \pm 1.3$ & $2.3 \pm 1.3$ \\
\hline PASAT & $49.8 \pm 4.5$ & $50.5 \pm 6.4$ & $54.2 \pm 3.7^{*}$ \\
\hline NHPT dominant hand (s) & $20.2 \pm 4.1$ & $19.5 \pm 3$ & $19.3 \pm 2.7$ \\
\hline NHPT non-dominant hand (s) & $20.1 \pm 3.3$ & $20.2 \pm 2.3$ & $19.7 \pm 3$ \\
\hline T25FW (s) & $4.7 \pm 1.8$ & $5.1 \pm 2.4$ & $5.3 \pm 2.2$ \\
\hline FSMC (motor) & $31.8 \pm 6.3$ & $32.2 \pm 6.2$ & $32.4 \pm 6.6$ \\
\hline FSMC (cognitive) & $34.2 \pm 9.7$ & $32.7 \pm 8.6$ & $33.4 \pm 9.6$ \\
\hline \multicolumn{4}{|l|}{$\begin{array}{l}\text { Absolute number }\left(\times 10^{3} / \mathrm{ml}\right)^{1} \\
\text { (clinic) }\end{array}$} \\
\hline Leukocytes & $7655 \pm 1870$ & $6763 \pm 2577$ & $6782 \pm 2707$ \\
\hline Lymphocytes & $2263 \pm 765$ & $1816 \pm 676$ & $1293 \pm 461^{*}$ \\
\hline Monocytes & $503 \pm 145$ & $484 \pm 130$ & $509 \pm 189$ \\
\hline Neutrophils & $4701 \pm 1244$ & $4276 \pm 1779$ & $4817 \pm 2290$ \\
\hline \multicolumn{4}{|l|}{$\begin{array}{l}\text { Absolute number }\left(\times 10^{3} / \mathrm{ml}\right)^{1} \\
\text { (calculated) }\end{array}$} \\
\hline CD56 ${ }^{\text {dim }}$ NK cells & $174 \pm 88$ & $137 \pm 93$ & $98 \pm 52 * *$ \\
\hline $\mathrm{CD} 6^{+} \mathrm{NK}$ cells & $21 \pm 12$ & $24 \pm 15$ & $20 \pm 10$ \\
\hline T cells $\left(\mathrm{CD}^{+}\right)$ & $1686 \pm 629$ & $1383 \pm 504^{*}$ & $956 \pm 417 * *$ \\
\hline $\mathrm{CD}^{+}{ }^{+} \mathrm{T}$ cells & $1002 \pm 313$ & $867 \pm 342$ & $645 \pm 317 * *$ \\
\hline $\begin{array}{l}\text { Naive CD4 }{ }^{+} \mathrm{T} \text { cells } \\
\left(\mathrm{CD}^{+} \mathrm{CD} 45 \mathrm{RO}^{-} \mathrm{CD} 45 \mathrm{RA}^{+}\right)\end{array}$ & $478 \pm 267$ & $487 \pm 239$ & $427 \pm 298$ \\
\hline $\begin{array}{l}\text { Memory CD4+ T cells } \\
\left(\text { CD4 }^{+} \text {CD45RO }{ }^{+} \text {CD45RA }\right)\end{array}$ & $518 \pm 184$ & $392 \pm 275^{*}$ & $216 \pm 158^{* * *}$ \\
\hline $\begin{array}{l}\text { Regulatory T cells } \\
\left(\mathrm{CD} 4^{+} \mathrm{CD} 25^{+} \mathrm{CD} 127^{\text {low }}\right)\end{array}$ & $43 \pm 19$ & $36 \pm 19$ & $28 \pm 17 *$ \\
\hline IL-17+ CD4 ${ }^{+} \mathrm{T}$ cells & $5 \pm 4$ & $3 \pm 3$ & $2 \pm 2 *$ \\
\hline $\mathrm{GM}^{-\mathrm{CSF}^{+} \mathrm{CD}^{+} \mathrm{T} \text { cells }}$ & $51 \pm 31$ & $35 \pm 37$ & $14 \pm 13^{* * *}$ \\
\hline IFNY- $\gamma^{+}$CD4+ T cells & $65 \pm 49$ & $43 \pm 41$ & $22 \pm 16 * *$ \\
\hline $\mathrm{IL}-4^{+} \mathrm{CD} 4^{+} \mathrm{T}$ cells & $21 \pm 18$ & $15 \pm 15$ & $12 \pm 11 *$ \\
\hline IL-10+ CD4 ${ }^{+} \mathrm{T}$ cells & $4 \pm 3$ & $4 \pm 4$ & $2 \pm 2 * * *$ \\
\hline $\mathrm{CD}^{+} \mathrm{T}$ cells & $522 \pm 365$ & $387 \pm 220 *$ & $228 \pm 132 * *$ \\
\hline $\begin{array}{l}\text { Naive CD8+ T cells } \\
\left(\text { CD8 }^{+} \text {CD45RO-CD45RA }{ }^{+}\right)\end{array}$ & $366 \pm 288$ & $271 \pm 157 \S$ & $194 \pm 114^{* *}$ \\
\hline $\begin{array}{l}\text { Memory CD8+ T cells } \\
\left(\text { CD8 }^{+} \text {CD45RO }{ }^{+} \text {CD45RA }\right)\end{array}$ & $152 \pm 152$ & $116 \pm 140 \S$ & $34 \pm 24 * * *$ \\
\hline B cells $\left(\mathrm{CD} 19^{+}\right)$ & $244 \pm 161$ & $154 \pm 109 * *$ & $124 \pm 59 * *$ \\
\hline $\begin{array}{l}\text { Transitional B cells }\left(\mathrm{CD} 19^{+} \mathrm{CD} 27^{-}\right. \\
\left.\mathrm{CD}^{-} 8^{+}\right)\end{array}$ & $7 \pm 5$ & $9 \pm 5$ & $9 \pm 7$ \\
\hline Naive B cells (CD19+IgD+CD27-) & $155 \pm 127$ & $104 \pm 75^{*}$ & $92 \pm 53^{* *}$ \\
\hline $\begin{array}{l}\text { Non class switched memory B } \\
\text { cells }\left(\mathrm{CD} 19^{+} \mathrm{IgD}^{+} \mathrm{CD} 27^{+}\right)\end{array}$ & $33 \pm 22$ & $17 \pm 7^{*}$ & $14 \pm 8 * * *$ \\
\hline $\begin{array}{l}\text { Class-switched memory B cells } \\
\left(\mathrm{CD} 19^{+} \mathrm{IgD}^{-} \mathrm{CD} 27^{+}\right)\end{array}$ & $34 \pm 16$ & $18 \pm 13^{* *}$ & $12 \pm 6 * * *$ \\
\hline $\begin{array}{l}\text { Double negative } B \text { cells } \\
(\text { CD19+IgD-CD27-) }\end{array}$ & $11 \pm 6$ & $5 \pm 4 * *$ & $4 \pm 2 * * *$ \\
\hline
\end{tabular}


$\mathrm{DMF}=$ dimethyl fumarate, $\mathrm{m}=$ months, $\mathrm{SD}=$ standard deviation, $\mathrm{S}=$ seconds, $\S \mathrm{p}=0.05, * \mathrm{p}<0.05, * * \mathrm{p}<0.01, * * * \mathrm{p}<0.001 ; 12 \mathrm{~m}$ compared to baseline ${ }^{1}$ Absolute numbers of immune cells were available from 12/21 DMF-treated MS patients, clinical tests were available from 14/21 DMF-treated MS patients.

\subsubsection{Percentages of innate immune cells increase after $12 \mathrm{~m}$ of DMF treatment}

When analysing innate immune cell subtypes (gated as in Figure 2.1(a)), increased percentages of monocytes $(p=0.0001)$ and $\operatorname{CD}^{+}(p=0.0001) \mathrm{NK}$ cells were detected after $12 \mathrm{~m}$ (Figure 2.1(b)), but not after $3 \mathrm{~m}$ (Supplementary Figure 2.1) of DMF treatment. Furthermore, no effect of DMF treatment was detected on $\mathrm{CD}^{\mathrm{dim}}$ ( $\mathrm{p}=0.4319$ ) NK cells after $12 \mathrm{~m}$. Thus, DMF treatment increased the percentages of monocytes and CD56 ${ }^{+}$NK cells.

(a)
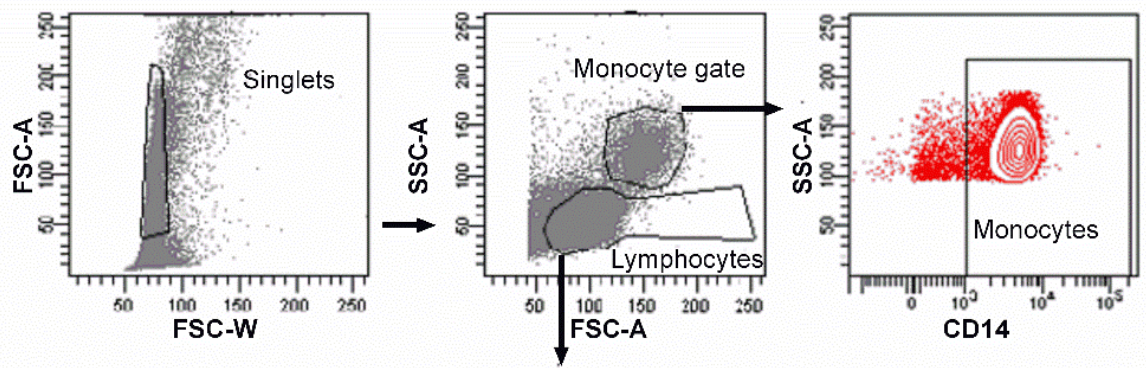

(b)
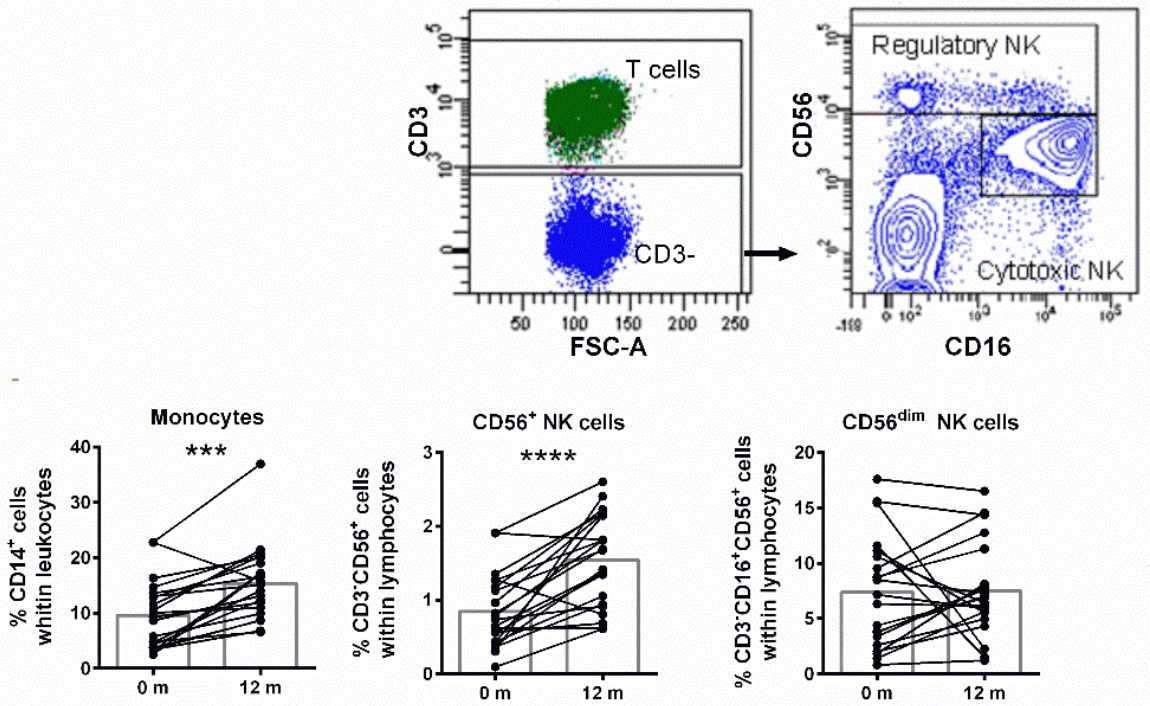


\section{Figure 2.1. DMF treatment increased percentages of monocytes and NK}

cells. (a) A representative analysis of innate immune cell subtypes is shown. Single cells were selected using forward scatter (FSC) area and width. Lymphocytes were gated in the singlet gate using FCS and side scatter (SSC) parameters. CD56 dim NK cells (CD3 $\left.\mathrm{CD} 16^{+} \mathrm{CD} 56^{\mathrm{dim}}\right)$ and $\mathrm{CD} 56^{+} \mathrm{NK}$ cells $\left(\mathrm{CD} 3^{-} \mathrm{CD} 16^{+} \mathrm{CD} 56^{+}\right)$were gated from lymphocytes and the $\mathrm{CD} 3$ - cell population. Monocytes $\left(\mathrm{CD} 14^{+}\right)$were gated from monocyte gate. (b) Frequency of monocytes and NK cells at baseline and after $12 \mathrm{~m}$ of DMF treatment in RRMS patients $(n=21)$. Each line represents an individual patient and average values are depicted as histograms. Wilcoxon matched-pairs signed rank test was used to determine $\mathrm{p}$ values. $* * * \mathrm{p}<0.001, * * * * \mathrm{p}<0.0001$. NK $=$ natural killer, $\mathrm{m}=$ months.

\subsubsection{Percentages of pro-inflammatory $T$ and $B$ cells decrease after 12 $\mathbf{m}$ of DMF treatment, while anti-inflammatory $T$ and $B$ cells increase}

Next, immune cell subtypes belonging to the adaptive immune system were examined following DMF treatment. After $3 \mathrm{~m}$ of DMF treatment no significant differences were observed in the percentages of these subtypes (Supplementary Figure 2.1). After $12 \mathrm{~m}$ of DMF treatment, no effect was shown on the percentages of T cells (data not shown). Within the T cell population (gated as in Figure 2.2(a)), $\mathrm{CD}^{+} \mathrm{T}$ cells remained stable, while $\mathrm{CD} 8^{+} \mathrm{T}$ cells decreased $(\mathrm{p}=0.0001$, Figure 2.2(b)). Most importantly, a decrease in the percentages of memory and effector memory $\mathrm{CD}^{+}$and $\mathrm{CD} 8^{+} \mathrm{T}$ cells was evident $(\mathrm{p}<0.0004)$ while naive $\mathrm{CD} 4^{+}$and $\mathrm{CD}^{+} \mathrm{T}$ cell percentages increased $(p<0.0001)$. No effect of DMF treatment was observed on Treg percentages (Supplementary Figure 2.1). 
(a)

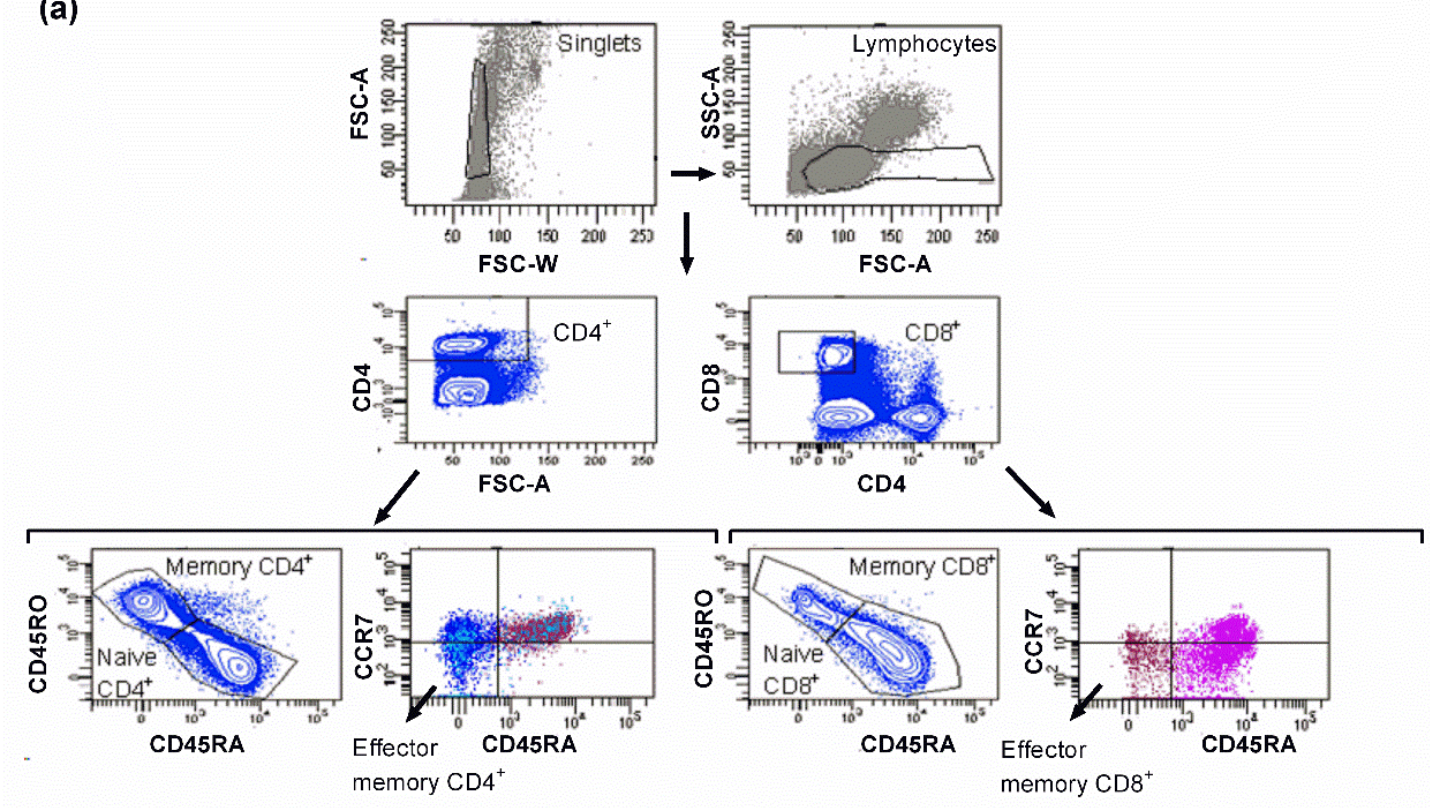

(b)
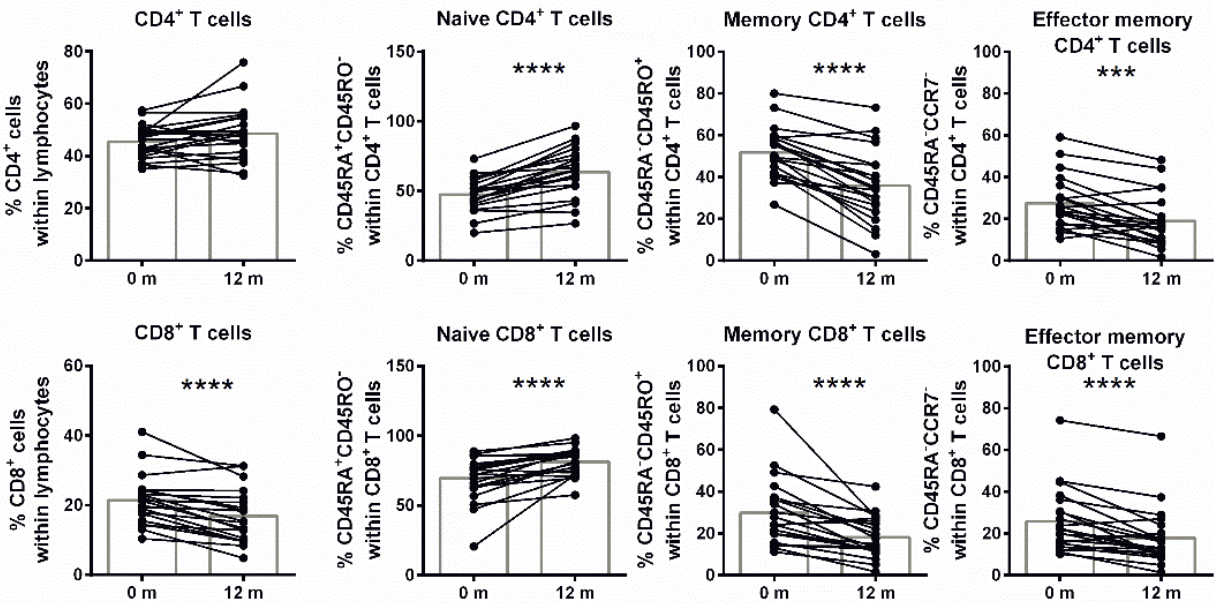

Figure 2.2. DMF treatment reduced (effector) memory $T$ cell percentages after $12 \mathbf{~ m}$ of treatment in MS patients.

(a) A representative analysis of $T$ cell subtypes is shown. Single cells were selected using forward FSC area and width. Lymphocytes were gated within the singlet gate using FCS and SSC parameters. Next, $C D 4^{+} \mathrm{T}$ cells and $\mathrm{CD} 8^{+} \mathrm{T}$ cells were gated from lymphocytes. Naive T cells (CD45RA $\left.{ }^{+} \mathrm{CD}^{2} 5 \mathrm{RO}^{-}\right)$, memory T cells $\left(\mathrm{CD}^{-} 5 \mathrm{RA}^{-} \mathrm{CD} 45 \mathrm{RO}^{+}\right)$and effector memory $\mathrm{T}$ cells 
(CD45RA ${ }^{-} C C R 7^{-}$) were gated from the $\mathrm{CD}^{+}$or $\mathrm{CD}^{+} \mathrm{T}$ cell population. (b) Frequency of $\mathrm{T}$ cell subtypes at baseline and after $12 \mathrm{~m}$ of DMF treatment in RRMS patients $(n=21)$. Each line represents an individual patient and average values are depicted as histograms. Wilcoxon matched-pairs signed rank test was used to determine $\mathrm{p}$ values. ${ }^{* * *} \mathrm{p}<0.001$, $* * * * \mathrm{p}<0.0001 . \mathrm{m}=$ months.

When analysing cytokine-expressing CD4 ${ }^{+} \mathrm{T}$ cells (gating shown in Supplementary Figure 2.2), decreased percentages of pro-inflammatory IFN- $\gamma^{+}(p=0.0014)$ and $\mathrm{GM}^{-\mathrm{CSF}^{+}}(\mathrm{p}<0.0001) \mathrm{CD}^{+} \mathrm{T}$ cells (Figure 2.3(a)) were detected after $12 \mathrm{~m}$ of DMF treatment while a trend was detected towards a decrease in IL-17+ ${ }^{+} D 4^{+} \mathrm{T}$ cells $(p=0.0568)$. Anti-inflammatory $\mathrm{IL}_{-} 4^{+}$and $\mathrm{IL}_{-10^{+}} \mathrm{CD}^{+}{ }^{+} \mathrm{T}$ cells were unchanged after $12 \mathrm{~m}$ of DMF treatment (Figure 2.3(b), Supplementary Figure 2.2). Thus, DMF treatment decreased pro-inflammatory cytokine expressing CD4+ cells without having an effect on anti-inflammatory cytokine expressing CD4 $^{+}$ cells.

(a)
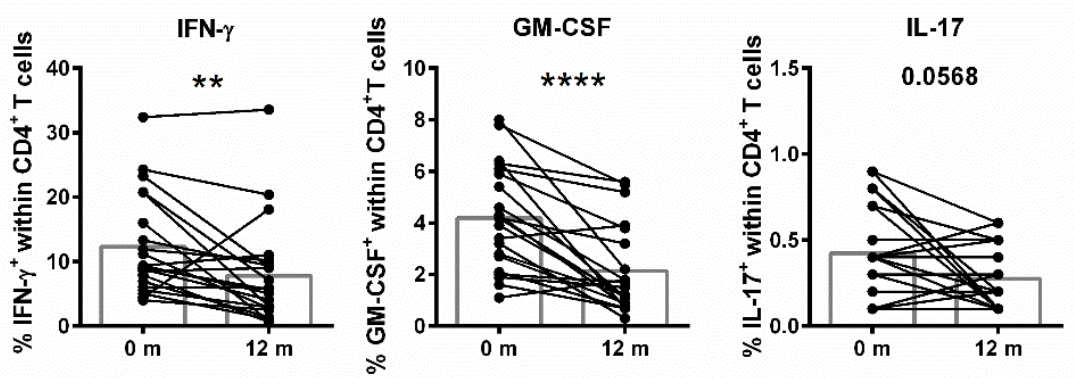

(b)
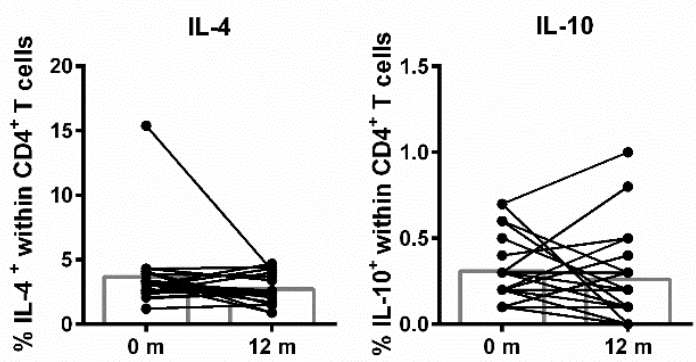

Figure 2.3. Reduction in the percentages of $\mathbf{T}$ cells expressing proinflammatory cytokines after $12 \mathrm{~m}$ of DMF treatment. Frequency of $\mathrm{CD}^{+} \mathrm{T}$ cells expressing pro-inflammatory cytokines (a), including IFN- $\gamma$, GM-CSF, IL-17 or antiinflammatory cytokines (b), including IL-4 and IL-10, in RRMS patients at baseline and after $12 \mathrm{~m}$ of DMF treatment $(\mathrm{n}=21)$. Each line represents an individual patient and average values are depicted as histograms. Wilcoxon matched-pairs signed rank test was used to determine $\mathrm{p}$ values. $* * \mathrm{p}<0.01, * * * * \mathrm{p}<0.0001$. IFN $-\mathrm{Y}=$ interferon $\mathrm{Y}, \mathrm{GM}-\mathrm{CSF}=$ 
granulocyte macrophage colony-stimulating factor, IL-17 = interleukin-17, IL-4 = interleukin-4, IL-10 = interleukin-10, $\mathrm{m}=$ months.

Furthermore, B cell percentages were not affected by DMF treatment (Figure 2.4). Within the $B$ cell population (gated as in Figure 2.4(a)), percentages of transitional and naive $B$ cells increased $(p=0.0007$ and $p<0.0001$, respectively, Figure 2.4(b)) after $12 \mathrm{~m}$ of DMF treatment. Interestingly, the increase in percentages of transitional B cells was already detected after $3 \mathrm{~m}$ of DMF treatment ( $p<0.05$, Supplementary Figure 2.1). Further, DMF treatment resulted in decreased percentages of DN ( $p=0.0014)$, class-switched memory $(p=0.0010)$ and non class-switched memory ( $p=0.0019$ ) B cells. Moreover, the frequencies of IgG $^{+}$ and $\mathrm{IgA}^{+}$class-switched memory $\mathrm{B}$ cells were unchanged after $12 \mathrm{~m}$ of DMF treatment (Supplementary Figure 2.1). Together, these results indicate that $12 \mathrm{~m}$ DMF treatment reduced percentages of pro-inflammatory and memory $T$ and $B$ cell subtypes and increased percentages of naive $T$ and $B$ cells and transitional $B$ cells. 
(a)
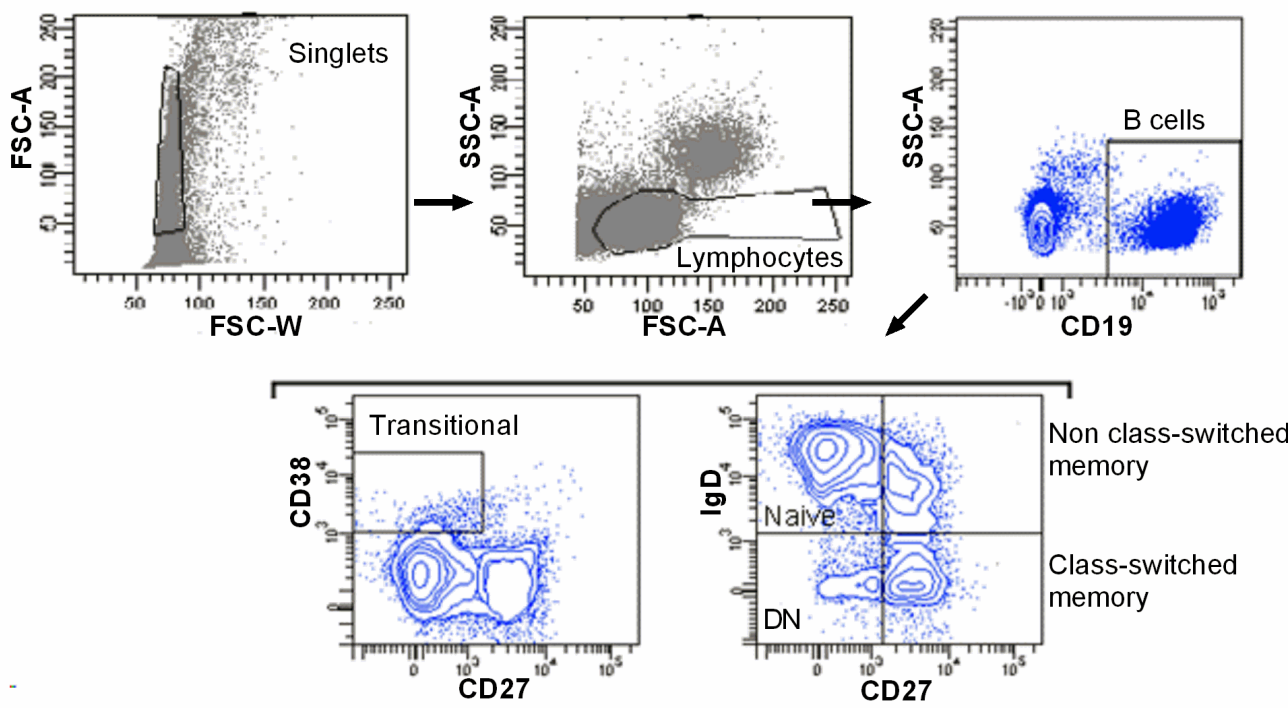

(b)
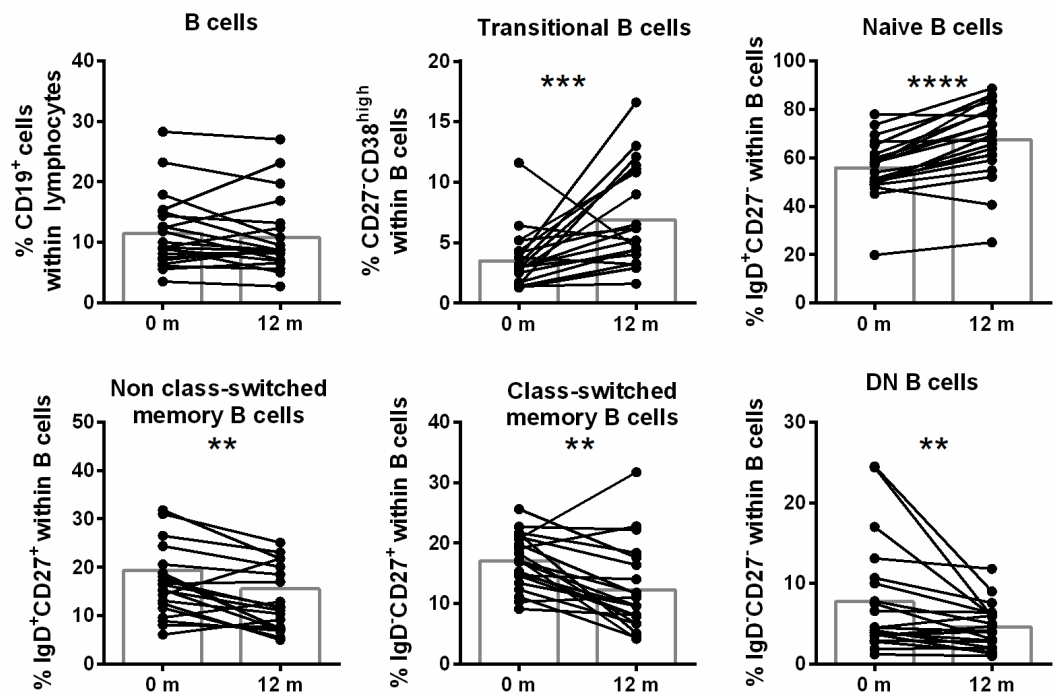

Figure 2.4. DMF treatment reduced percentages of memory and DN B cell subtypes after $12 \mathrm{~m}$ of treatment in MS patients. (a) A representative analysis of B cell subtypes is shown. Single cells were selected using FSC area and width. Lymphocytes were gated in the singlet gate using FCS and SSC parameters. B cells (CD19+) were gated from lymphocytes followed by analysis of transitional B cells $\left(C D 27^{-C D} 38^{\text {high }}\right)$, naive $B$ cells $\left(\operatorname{IgD}^{+} \mathrm{CD} 27^{-}\right)$, non class-switched memory $B$ cells $\left(\operatorname{IgD}{ }^{+} \mathrm{CD} 27^{+}\right)$, class-switched 
memory B cells ( $\left.\operatorname{IgD}^{-} \mathrm{CD} 27^{+}\right)$and $\mathrm{DN} B$ cells (IgD-CD27-). (b) B cell subtypes at baseline and after $12 \mathrm{~m}$ of DMF treatment in RRMS patients $(n=21)$. Each line represents an individual patient and average values are depicted as histograms. Wilcoxon matched-pairs signed rank test was used to determine $\mathrm{p}$ values. $* * \mathrm{p}<0.01, * * * \mathrm{p}<0.001, * * * * \mathrm{p}<0.0001$. DN $=$ double negative, $\mathrm{m}=$ months.

\subsubsection{DMF exerts its full effect on immune cells after $6 \mathrm{~m}$ of treatment in a cross-sectional study}

Limited information is available concerning the point in time when DMF is fully effective in terms of immunology. Since $3 \mathrm{~m}$ DMF treatment only partly reflected changes reported at $12 \mathrm{~m}$, additional time points were included in a crosssectional study to identify how soon the reported effect was found after treatment (Table 2.1). Memory $C D 4^{+}$and $C D 8^{+} T$ cell percentages were reduced $(p=0.0025$ and $p=0.0001$, respectively), while naive $C D 4^{+}$and $C D 8^{+} T$ cell percentages were increased ( $p=0.0022$ and $p<0.0001$, respectively) after $6 \mathrm{~m}$ of DMF treatment compared to untreated MS patients (Figure 2.5). Furthermore, percentages of memory $\mathrm{CD}^{+} \mathrm{T}$ cells were decreased $(\mathrm{p}=0.0079)$, while naive $\mathrm{CD} 8^{+} \mathrm{T}$ cells were increased ( $p=0.0072$, Figure 2.5) in MS patients treated with DMF for $6-12 \mathrm{~m}$ compared with MS patients treated with DMF for 1-5 m. After prolonged treatment (> $12 \mathrm{~m}$ ), memory and naive $\mathrm{CD}^{+}$and $\mathrm{CD}^{+} \mathrm{T}$ cell percentages remained stable. Thus, DMF exerts its full effect on immune cells after $6 \mathrm{~m}$ of treatment. 
(a)

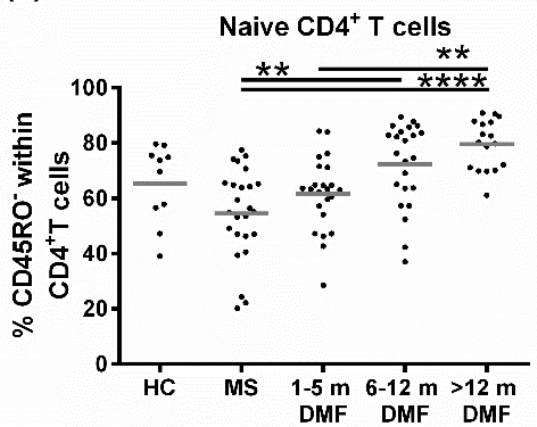

(c)

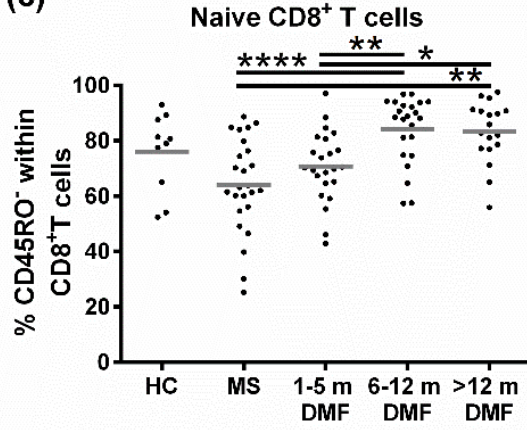

(b)

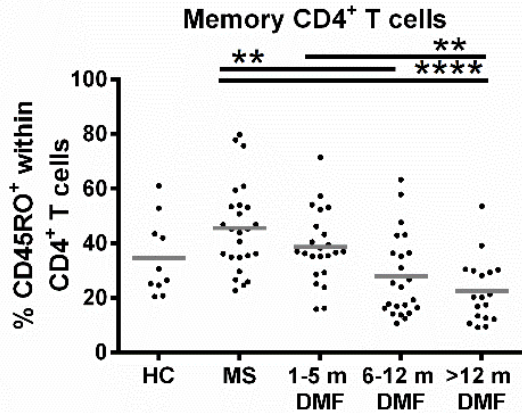

(d)

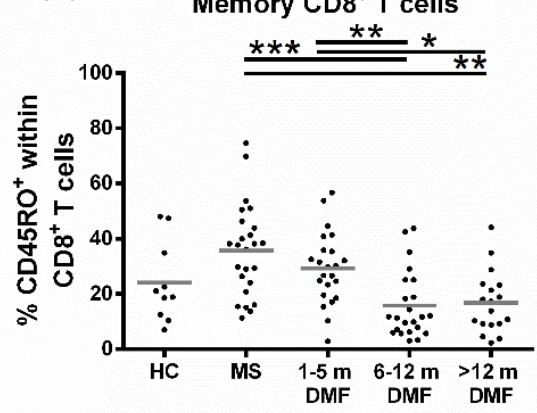

Figure 2.5. DMF treatment is fully effective on immune cells after $\mathbf{6} \mathbf{m}$ of treatment. Frequencies of naive and memory $\mathrm{CD}^{+}$and $\mathrm{CD}^{+} \mathrm{T}$ cells in $\mathrm{HC}(\mathrm{n}=10)$, untreated RRMS patients ( $n=25), 1-5 \mathrm{~m}$ DMF treated RRMS patients $(n=23), 6-12 \mathrm{~m}$ DMF treated RRMS patients $(n=23),>12$ m DMF treated MS patients $(n=18)$. A KruskalWallis one-way ANOVA was used to compare the different groups. $* \mathrm{p}<0.05, * * p<0.01$, $* * * \mathrm{p}<0.001, * * * * \mathrm{p}<0.0001$. HC $=$ healthy control, DMF = dimethyl fumarate, $\mathrm{m}=$ months.

\subsubsection{No effect of DMF treatment on functional markers and survival markers of B cells of RRMS patients}

B cells are able to activate $\mathrm{T}$ cells via effective antigen presentation and costimulation. Our research group previously reported that peripheral blood $\mathrm{B}$ cells of untreated MS patients express higher levels of costimulatory molecules than B cells of HC (123). Since the effect was only observed in untreated MS patients, it is important to investigate if DMF treatment can restore the expression of antigen presentation and costimulatory molecules as well. 
In the follow-up study, no unambiguous effect of DMF treatment was detected on the expression of antigen presentation molecule HLA-DR/DP/DQ or costimulatory molecules CD86, CD80 and CD40 on B cells after $12 \mathrm{~m}$ of treatment (Figure 2.6(a)). Furthermore, BAFF and APRIL are two cytokines important for the proliferation and survival of $B$ cells. In the brain of MS patients, BAFF and APRIL are elevated compared to $\mathrm{HC}(136,296)$. Therefore, it is interesting to investigate if DMF treatment could downregulate the level of BAFF in the plasma of MS patients and the expression of survival receptors for these cytokines on $B$ cells in MS patients. After $12 \mathrm{~m}$ of DMF treatment, the expression levels of BAFFR, TACI and BCMA on $B$ cells of MS patients and the BAFF plasma levels of MS patients were unchanged (Figure 2.6(b, c)). In conclusion, functional and survival markers of $B$ cells of MS patients were not affected by DMF treatment.

(a)
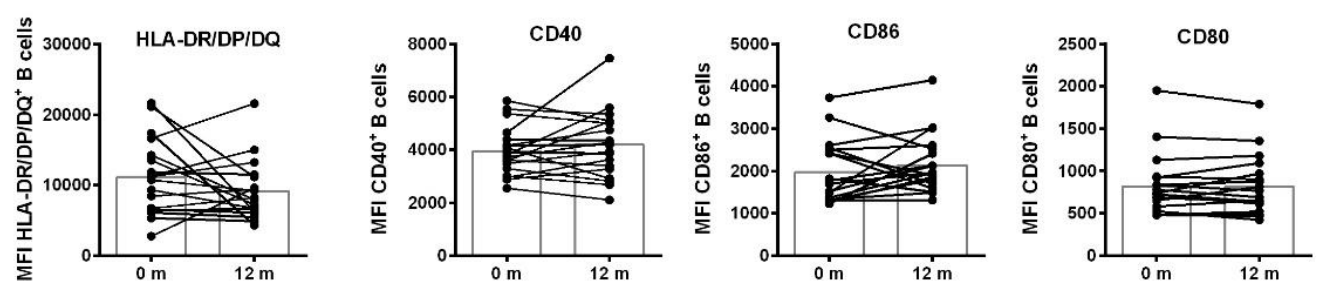

(b)
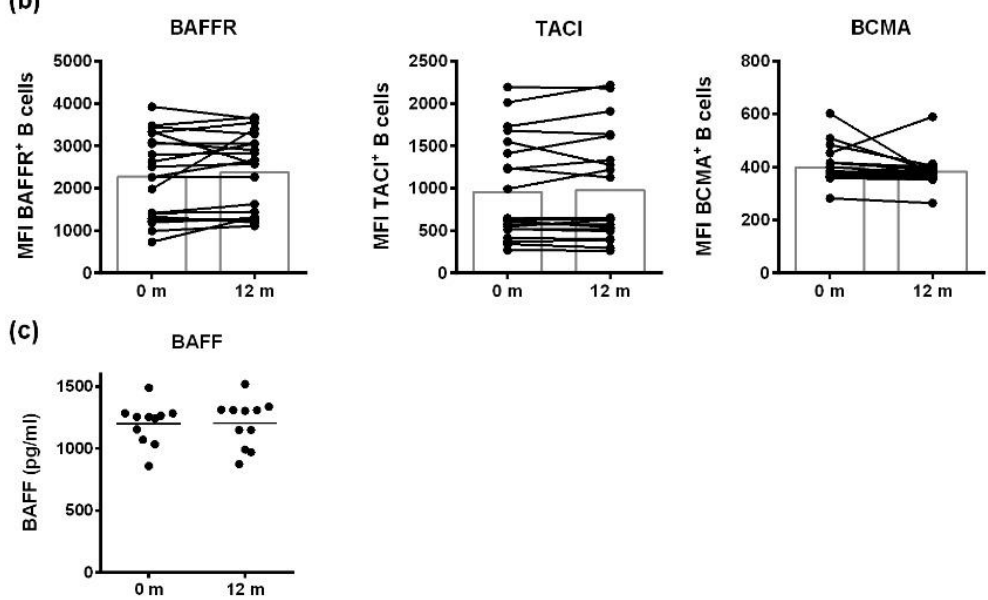

Figure 2.6 DMF treatment does not influence the expression of antigenpresentation molecules, costimulatory molecules and survival markers on B cells.

(a) Expression (MFI) of antigen-presenting molecule (HLA-DR/DP/DQ) and costimulatory 
molecules CD40, CD86 and CD80 on B cells at baseline and after $12 \mathrm{~m}$ of DMF treatment in RRMS patients $(n=21)$. (b) Expression (MFI) of survival markers BAFFR, TACI and BCMA on $B$ cells at baseline and after $12 \mathrm{~m}$ of DMF treatment in RRMS patients $(n=21)$. Each line represents an individual patient and average values are depicted as histograms. (c) Plasma BAFF levels of RRMS patients at baseline and $12 \mathrm{~m}$ of DMF treatment $(n=11)$. Wilcoxon matched-pairs signed rank test was used to determine $\mathrm{p}$ values. HLA-DR/DP/DQ = human leukocyte antigen, MFI = mean fluorescence intensity, $\operatorname{BAFF}(R)=B$ cell activating factor (receptor), TACI $=$ transmembrane activator and calcium modulator and cyclophilin ligand interactor, $\mathrm{BCMA}=\mathrm{B}$-cell maturation antigen. 


\subsection{Discussion}

In 2013, DMF (Tecfidera ${ }^{\circledR}$ ) was licensed as an oral first-line treatment for RRMS $(201,202)$. However, its mechanism of action is still not fully elucidated. We demonstrated that DMF treatment induced a persistent change of both the innate and adaptive immune system of MS patients.

Next to the immunological findings, clinical parameters were analysed in the 12 $\mathrm{m}$ follow-up study. DMF treatment improved cognitive functions, namely auditory processing speed and attention (PASAT). Cognitive decline is a serious complication in MS that is characterized by deficits in (non)verbal memory, information processing speed and sustained attention (297). However, validation in a larger cohort of DMF-treated MS patients is needed. Furthermore, in order to fully explore the improvement in cognitive function, it would be advised to perform the other cognitive tests of the brief repeatable neuropsychological tests, and not only the PASAT $(298,299)$.

The present study investigated the absolute numbers and the frequencies of immune cell subtypes of the innate and the adaptive immune system in order to evaluate both potential immune suppression and immune cell subtype distribution in the remaining cell population. We showed that DMF treatment selectively targets or has a stronger effect on particular immune cell subtypes, since it decreased total lymphocyte, $T$ cell and $B$ cell numbers without affecting monocytes, neutrophils and anti-inflammatory subtypes such as CD56+ ${ }^{+}$KK cells and transitional $B$ cells. Absolute numbers in this study are comparable to others (300-303). The B cell subtype numbers are in line with Li et al. where DMF preferentially decreased mature B cells without affecting transitional B cells after at least $3 \mathrm{~m}$ of treatment (295). However, our study reinforced this by showing a persistent change after $12 \mathrm{~m}$. We further confirmed the preferential loss of CD8 ${ }^{+}$ T cells (56\%) versus CD4 ${ }^{+} \mathrm{T}$ cells (36\%) and additionally indicated that this loss was persistent after $12 \mathrm{~m}$ of treatment. However, in our study, 25\% (3/12) of the DMF-treated MS patients had a decline in absolute $\mathrm{CD} 4^{+} \mathrm{T}$ cell numbers below the lower limit of normal (LLN, 400/ $\mu \mathrm{l}$ ) compared to $31 \%(13 / 42)$ in the study of Khatri et al. (302). Furthermore, 42\% (5/12) of the DMF-treated MS patients had a decline of $\mathrm{CD}^{+} \mathrm{T}$ cells below the LLN (200/ $\left.\mu \mathrm{l}\right)$ compared to 54\% (21/39) in the study of Khatri et al. and to the majority of the patients in the study of Spencer 
et al. $(302,303)$. The lower percentages in our study could be due to difference in patient numbers and inclusion criteria $(302,303)$.

As the absolute numbers of almost all immune cell subtypes decreased, it was important to consider their percentages to elucidate which subtypes were preferentially affected by DMF treatment. We showed that innate immune cell percentages (monocytes and $\mathrm{CD}^{+} 6^{+} \mathrm{NK}$ cells) increased, implying that DMF prevails normal innate immune function. The relative increase in monocytes and CD56+ NK cells could be the result of the decreased absolute lymphocyte number, as the absolute monocyte and CD56+ NK cell numbers did not change. The relative unchanged percentage of CD56 $\mathrm{dim}$ NK cells could also be the result of the decreased absolute lymphocyte number since CD56 dim NK cells decreased in absolute number. In agreement with our study, Medina et al. demonstrated an increase of $\mathrm{CD}^{2} 6^{+} \mathrm{NK}$ cells after $6 \mathrm{~m}$ of DMF treatment. Nonetheless, our study reinforces this result by showing a persistent increase after $12 \mathrm{~m}$ of DMF treatment (304). Furthermore, Chaves et al. and Medina et al. did not find a difference in NK cell percentages, which indicates that it is important to investigate the subtypes of NK cells since both subtypes have different functions $(304,305)$. NK cells have become increasingly important as players in MS pathogenesis, as the expansion of $\mathrm{CD}^{2} 6^{+} \mathrm{NK}$ cells was previously correlated with the efficacy of daclizumab in MS patients (306). CD56 ${ }^{+}$NK cells were first considered regulatory NK cells by Cooper et al. due to a reduced cytotoxicity and an increased production of cytokines compared to cytotoxic CD56 dim NK cells (78). Regulatory NK cells cytolyze activated T cells in a comparable way as cytotoxic NK cells, although they display no cytotoxicity towards resting T cells (306). As with daclizumab, the increase in regulatory NK cells is one of the beneficial mechanisms of DMF. Other mechanisms of DMF, including $T$ cell apoptosis and inhibition of dendritic cell maturation, might indirectly be a consequence of this finding $(301,307)$.

Within the adaptive immune system, DMF treatment reduced percentages of proinflammatory immune cell subtypes such as (effector) memory (CD4 ${ }^{+}$and $\mathrm{CD}^{+}$) T cells and (non) class-switched memory and DN B cells in the follow-up study. We previously demonstrated that age-associated DN B cells were increased in the blood and cerebrospinal fluid of a proportion MS patients (68). The decreased frequency of DN B cells following DMF treatment could have beneficial effects in 
MS pathogenesis as DN B cells showed pro-inflammatory functional capacities (68). Furthermore, DMF treatment increased naive $\mathrm{T}$ and $\mathrm{B}$ cells and transitional $B$ cells.

This switch from a pro-inflammatory profile into an anti-inflammatory profile is in line with other studies (199, 304, 308, 309). Since the same results were obtained in different study populations originating from different countries (Spain, Germany, USA and Belgium), it appears that DMF has a rather stable immunological effect, although it has many mechanisms of actions. In addition, our cross-sectional study emphasizes the long-lasting effect of DMF. Medina et al. and Smith et al. reported a reduction of memory $B$ cells while naive $B$ cells increased at $6 \mathrm{~m}$ of treatment $(304,310)$. However, no effect of DMF was observed on transitional and Breg cells after $6 \mathrm{~m}$ of treatment $(304,310)$. Our study reinforces this result by showing a persistent effect of DMF after $12 \mathrm{~m}$ of treatment on naive and memory B cells. However, in our study we detected an increase in transitional B cells. This finding is also shown in the study of Lundy et al., where in 4 of the 8 patients Breg cells have increased even more after 12 months of DMF treatment compared to 4 to 6 months of DMF treatment (311). Furthermore, we can speculate that transitional and naive $B$ cells are more resistant to DMF treatment than memory $B$ cells.

Furthermore, we did not detect a change in Treg cells, which is in line with the studies of Medina et al. $\left(C D 4^{+} \mathrm{CD} 127^{\text {low }} \mathrm{CD} 25^{+}\right)$, Ghadiri et al. $\left(\mathrm{CD} 4^{+} \mathrm{CD} 127^{\text {low }} \mathrm{CD} 25^{+}\right)$and Longbrake et al. $\left(\mathrm{CD} 4^{+} \mathrm{CD} 25^{+}\right.$Foxp3 $\left.{ }^{+}\right)(301,304,308)$. Using a different phenotyping of Treg $\left(C D 4^{+} \mathrm{CD} 127^{\text {low }} \mathrm{CD} 25^{\text {high }} \mathrm{FoxP} 3^{+} \mathrm{Helios}^{-}\right)$Gross et al. found an increase in these cells after DMF treatment. More research is needed to evaluate different subtypes of Tregs (199).

When investigating the effect of DMF treatment on the pro-inflammatory profile of $\mathrm{CD}^{+}{ }^{+} \mathrm{T}$ cells, we detected a reduction of IFN- $\mathrm{Y}^{+}$and $\mathrm{GM}-\mathrm{CSF}^{+} \mathrm{CD}^{+}{ }^{+} \mathrm{T}$ cells after $12 \mathrm{~m}$ of DMF treatment which is consistently reported in other studies $(199,301$, 309 , 312). However, Medina et al. did not detect an effect of DMF on GM-CSF after $6 \mathrm{~m}$ of treatment (304). In addition, the trend towards a decrease of $\mathrm{IL}-17^{+}$ $\mathrm{CD}^{+} \mathrm{T}$ cells detected in our study after $12 \mathrm{~m}$ of therapy is in accordance with the study of Wu et al., while Gross et al. and Medina et al. did not detect an effect after $6 \mathrm{~m}$ (199, 304, 309). Our study, together with the study of Wu et al. and 


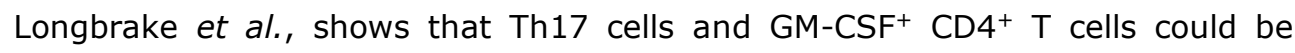
reduced after a longer period of DMF therapy (309).

When considering the anti-inflammatory cytokines IL-4 and IL-10, we could not find a difference after treatment with DMF. One explanation could be the great patient diversity within the population. Because of this variety no population-wide conclusion could be drawn. Similar to us, Medina et al., Longbrake et al. and Gross et al. did not detect a difference in the percentage of $\mathrm{IL}-4^{+}$or $\mathrm{IL}-10^{+} \mathrm{CD} 4^{+} \mathrm{T}$ cells $(199,312)$. In contrast, Wu et al. detected an increase in IL-4 ${ }^{+}$CD4 ${ }^{+}$T cells, however the percentage of $\mathrm{IL}-4^{+} \mathrm{CD} 4^{+} \mathrm{T}$ cells is much lower than in the other studies (309).

When analyzing the expression of functional molecules on B cells, we demonstrated that DMF treatment did not change the expression of antigen presentation molecules, costimulatory molecules and survival markers on B cells of MS patients. Compared with naive B cells, memory B cells are known to express higher levels of costimulatory molecules, so the shift to a more naive than memory $B$ cell phenotype after DMF treatment may be the reason why no effect of DMF could be detected on functional markers of total B cells of MS patients $(313,314)$.

In conclusion, this study shows that DMF treatment persistently changes the immune balance of MS patients and illustrates the multifactorial working mechanism of DMF. The effects of DMF elucidated here could help explain its therapeutic efficacy in MS. 


\subsection{Supplementary material}

(a)

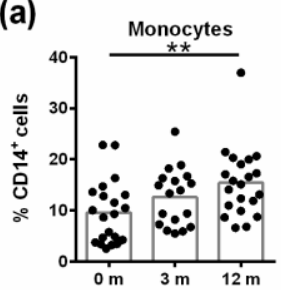

(b)
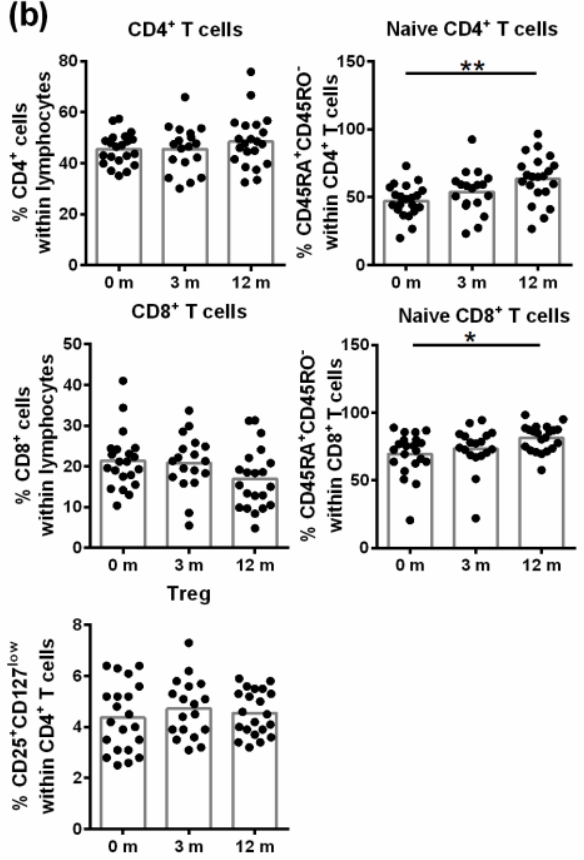

(c)
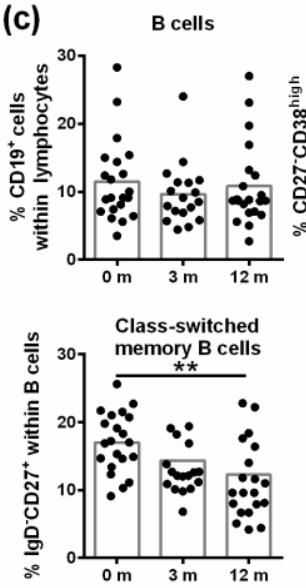
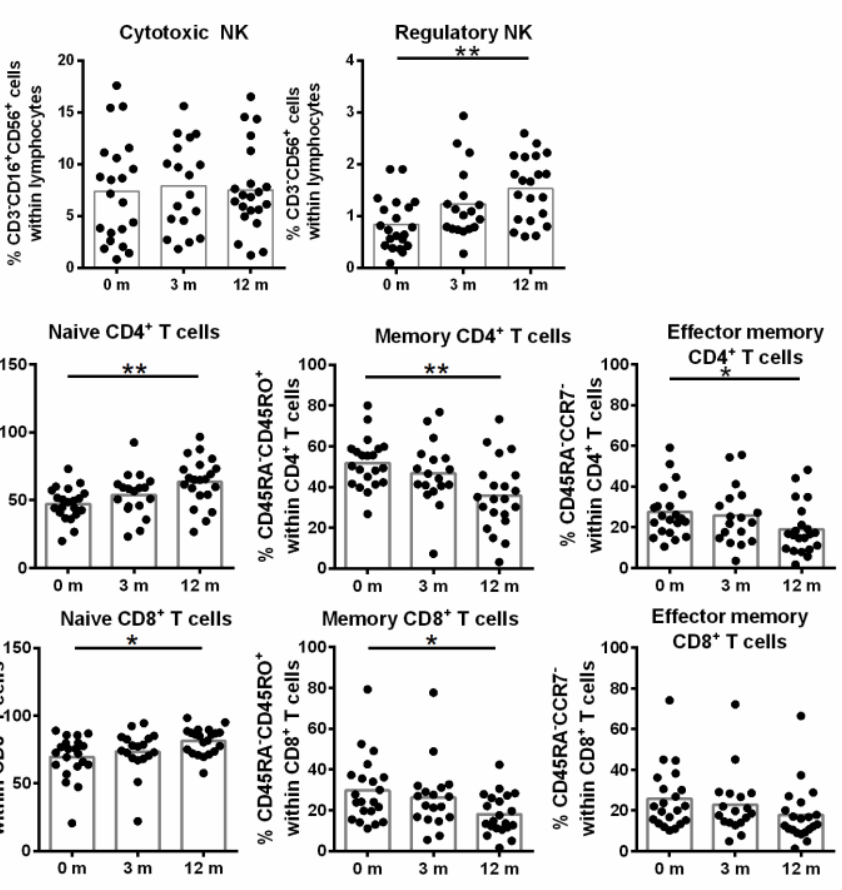
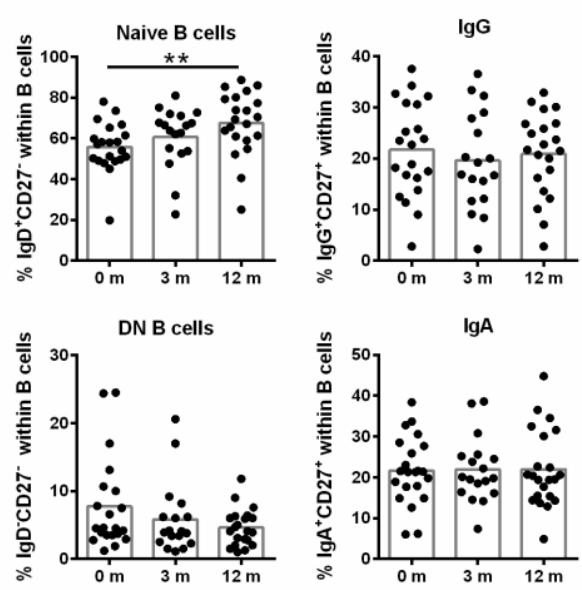
Supplementary Figure 2.1. DMF treatment induced redistribution of innate and adaptive immune cell subtypes after $12 \mathrm{~m}$ of treatment in RRMS patients, while almost no significant changes were detected at $\mathbf{3} \mathbf{~ m}$ of DMF treatment. (a) Frequency of monocytes, NK cells, (b) T cell subtypes and (c) B cell subtypes at baseline $(n=21)$, after $3 \mathrm{~m}(n=18)$ and after $12 \mathrm{~m}(n=21)$ of DMF treatment in RRMS patients. Each dot represents an individual patient and average values are depicted as histograms. A Kruskal-Wallis one-way ANOVA test was used to determine $p$ values. $* \mathrm{p}<0.05, * * \mathrm{p}<0.01$. NK $=$ natural killer, Treg $=$ regulatory $\mathrm{T}$ cells, $\mathrm{DN}=$ double negative, $\mathrm{m}=$ months. 
(a)
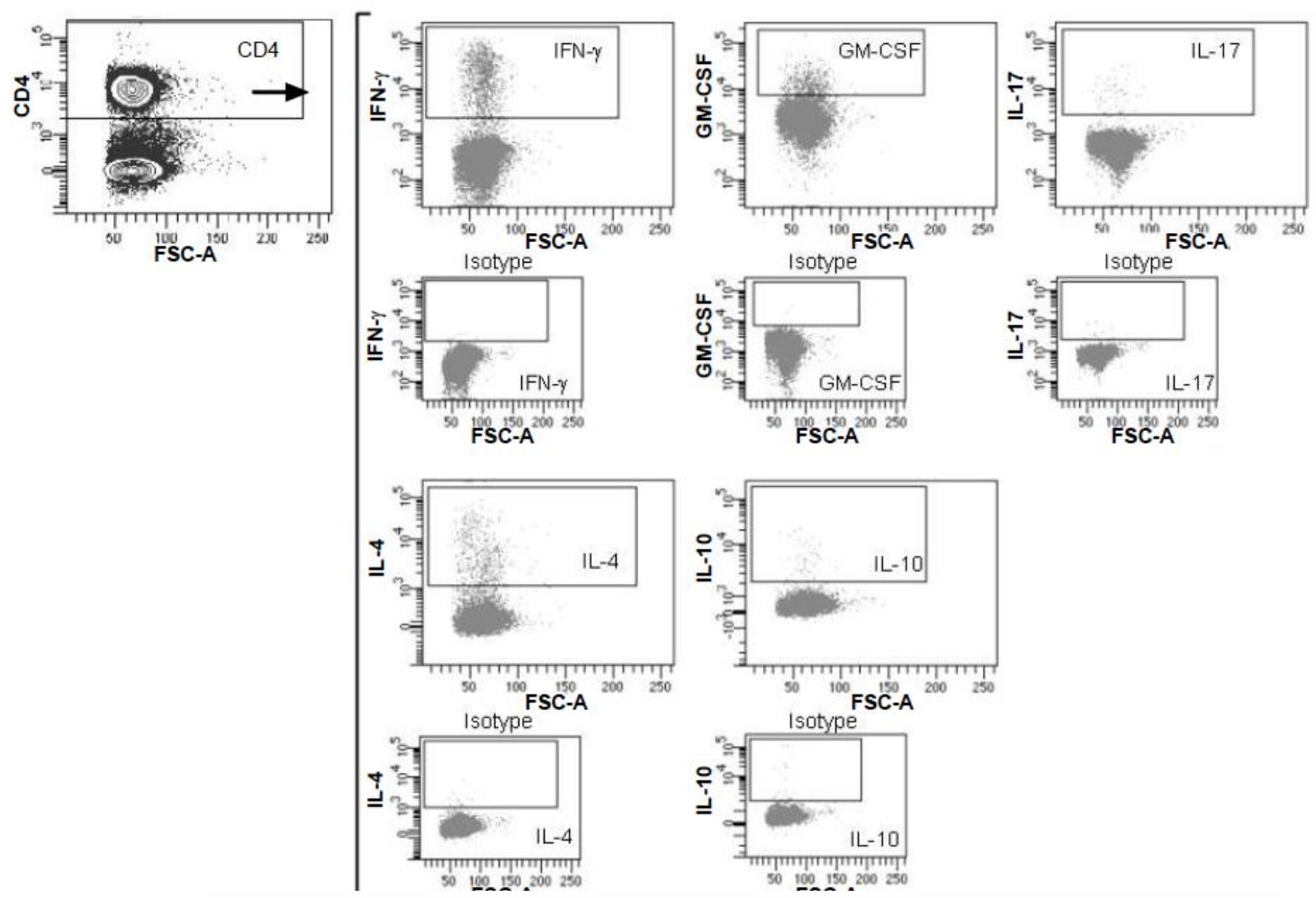

(b)
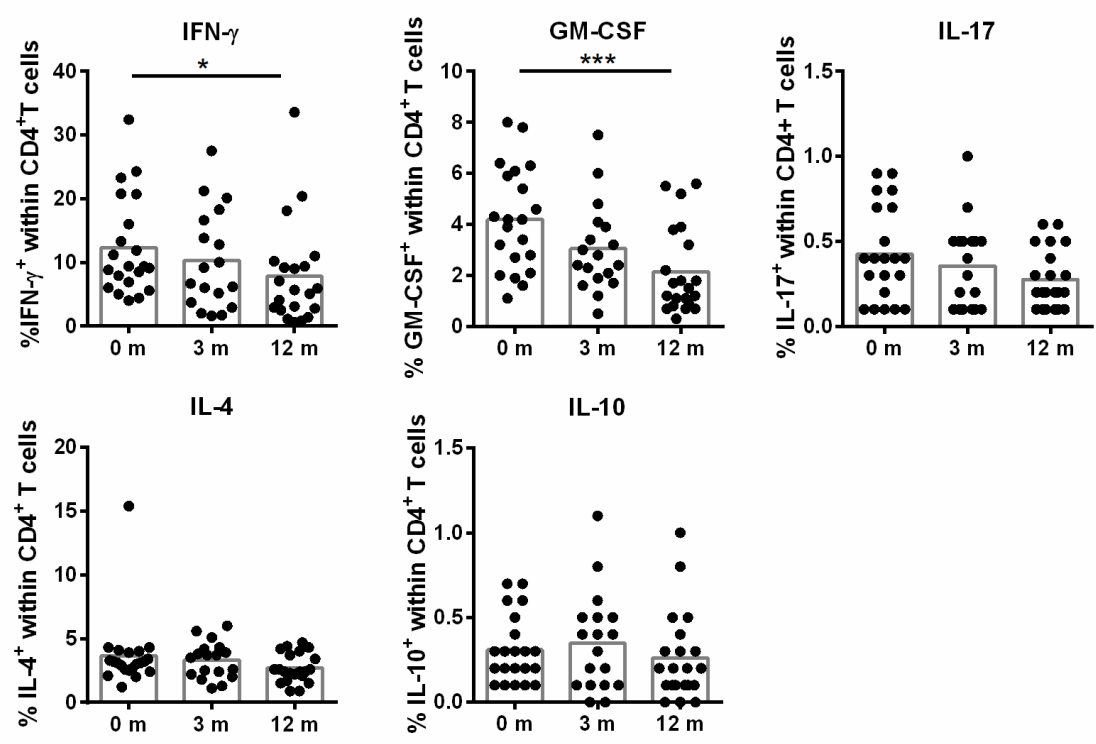


\section{Supplementary Figure 2.2. DMF treatment reduced frequency of $T$ cells} expressing pro-inflammatory cytokines after $12 \mathrm{~m}$, but not after $3 \mathbf{m}$ of treatment. (a) A representative analysis of cytokine-expressing $T$ cell subtypes is shown. $\mathrm{CD}^{+}{ }^{+} \mathrm{T}$ cells were gated from lymphocytes. IFN- $\mathrm{\gamma}^{+}, \mathrm{GM}-\mathrm{CSF}^{+}, \mathrm{IL}-17^{+}, \mathrm{IL}-10^{+}$and $\mathrm{IL}-4^{+}$cells were gated from the $\mathrm{CD} 4^{+}$cell population based on isotype controls (b) Frequency of $\mathrm{CD} 4^{+}$ $T$ cells expressing pro-inflammatory cytokines (IFN-Y, GM-CSF, IL-17) and antiinflammatory cytokines (IL-4, IL-10) in RRMS patients at baseline $(n=21)$, after $3 \mathrm{~m}(\mathrm{n}=$ $18)$ and after $12 \mathrm{~m}(\mathrm{n}=21)$ of DMF treatment. Each dot represents an individual patient and average values are depicted as histograms. A Kruskal-Wallis one-way ANOVA test was used to determine $\mathrm{p}$ values. $* \mathrm{p}<0.05, * * * \mathrm{p}<0.001$. IFN- $\mathrm{\gamma}=$ interferon- $\mathrm{y}, \mathrm{GM}-\mathrm{CSF}=$ granulocyte macrophage colony-stimulating factor, IL-17 = interleukin-17, IL-4 = interleukin-4, IL-10 = interleukin-10, m = months. 


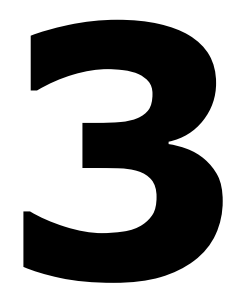

\section{DMF directly affects the expression of survival and functional markers of $B$ cells}

Based on:

Dimethyl fumarate induces a persistent change in the composition of the innate and adaptive immune system in multiple sclerosis patients Montes Diaz G., Fraussen J., Van Wijmeersch B., Hupperts R. and Somers V. Sci Rep. 2018 May 29;8(1):8194. 


\section{DMF directly affects the expression of survival and functional markers of B cells}

\subsection{Abstract}

Dimethyl fumarate (DMF; Tecfidera $®$ ) is licensed as the first oral therapy that can be prescribed as a first-line therapy to relapsing-remitting multiple sclerosis (RRMS) patients. B cells are essential contributors to the immunopathogenesis of MS. The mechanism of action of DMF is not fully clarified and little is known about the direct effect of DMF and its metabolite monomethylfumarate (MMF) on B cells. The aim of this study was to analyse the direct effects of DMF and MMF on B cells of untreated MS patients and healthy controls. Direct effects of DMF and MMF on B cell apoptosis (annexin V), expression of $B$ cell activating factor receptor (BAFFR) (HC $\mathrm{n}=14 ; \mathrm{MS} \mathrm{n}=6)$, expression of costimulatory molecules (CD40, CD80, CD86) and major histocompatibility complex - II (MHC-II) molecules (HC $\mathrm{n}=9 ; \mathrm{MS} \mathrm{n}=7$ ) and frequencies of regulatory $\mathrm{B}$ cells (Breg) and tumor necrosis factor-a $(T N F-a)^{+} B$ cells (MS $n=5$ ) were examined using in vitro cultures of purified B cells. DMF but not MMF induced apoptosis of B cells in a direct and concentration dependent manner. DMF decreased the expression of CD40, MHCII and BAFFR on B cells, which were not affected by MMF. Although not significant, a trend towards increased frequencies of Breg was induced by DMF while frequencies of TNF- $a+B$ cells were decreased by MMF. In conclusion, DMF induced apoptosis of $B$ cells, decreased the expression of functional B cell markers and increased the frequency of Breg, which could influence B cell function. 


\subsection{Introduction}

Multiple sclerosis (MS) is the major autoimmune-mediated neurodegenerative disease of the central nervous system (CNS), which leads to inflammation, demyelination, and axonal damage in the brain and spinal cord (1). Accumulating evidence has demonstrated that B cells play an essential role in MS, including most convincingly the beneficial clinical outcomes of B cell depletion therapies (97, 99). Several potential mechanisms have been proposed which contribute to reduced MS activity after $B$ cell depletion treatment, including repopulating $B$ cells consisting of larger numbers of naive $B$ cells and fewer antigen-educated memory $B$ cells and plasmablasts, a change in B cell cytokine production and a decrease in B cell antigen presentation capacity $(221,315)$.

MS patients present with an imbalance in the immune system with an increase of memory B cells secreting pro-inflammatory cytokines such as granulocyte macrophage colony-stimulating factor (GM-CSF), lymphotoxin-a (LT-a) and tumor necrosis factor-a (TNF-a) and an underrepresentation of regulatory $B$ cells (Breg) secreting cytokines such as IL-10 (100, 114, 115, 117). Next to producing cytokines, B cells play an important role as highly efficient antigen presenting cells (APCs). In MS patients, it has been shown that B cells can present myelin antigens to $T$ cells and induce pro-inflammatory $T$ cell responses (123). Furthermore, costimulatory molecules, such as CD40, CD80 and CD86, expressed on B cells, interact with their ligand on $\mathrm{T}$ cells and activate effector $\mathrm{T}$ cells. In MS patients, expression of CD80 and CD86 is elevated on B cells compared to B cells of healthy controls (HC) (123).

Dimethyl fumarate (DMF) is one of the newer oral treatment options approved for the treatment of RRMS $(201,202)$. Upon uptake, DMF is partly metabolized to monomethyl fumarate (MMF) in the gastrointestinal tract $(244,245)$. Although DMF is not detectable in the serum following DMF intake, long-lived glutathioneconjugates of DMF are detected in urine of DMF treated patients (246-249). Furthermore, both DMF and MMF can be effectively hydrolyzed by lymphocytes and monocytes which highlights the possibility that DMF and MMF can exert direct and indirect effects on immune cells (244). 
Earlier studies in RRMS patients treated with DMF for 12 months, showed that DMF treatment reduces the absolute number of $B$ cells and alters the distribution of several $B$ cell subtypes including a reduction in the percentage of memory $B$ cell subtypes $(316,317)$.

The mechanism of action of DMF is not fully clarified. However the direct effect of DMF and MMF on T cells were studied in several in vitro studies. For instance, DMF and MMF affects the cytokine profile of T cells of $\mathrm{HC}$ and non-treated MS patients by inducing the expression of anti-inflammatory T helper 2 cytokines such as IL4 and IL-5, while the production of inflammatory T helper 1 cytokine IFN- $Y$ was unaffected $(288,318)$. Furthermore, an in vitro study with T cells of HC illustrated that DMF, but not MMF, induce a dose- and time-dependent apoptosis of preferably activated human $\mathrm{T}$ cells by downregulating $\mathrm{Bcl}-2$ expression (319). In addition, DMF directly inhibits activation and proliferation of $\mathrm{T}$ cells of $\mathrm{HC}$, as shown by in vitro experiments (309). Nonetheless, little is known about the direct effect of DMF and MMF on B cells (288). To address this, we examined the direct effect of DMF and MMF on B cell apoptosis, expression of the survival receptor BAFFR, antigen presentation molecules and costimulatory molecules (CD40, CD80 and CD86) on B cells of HC and untreated RRMS patients. 


\subsection{Material and methods}

\subsubsection{Study subjects}

RRMS patients and HC were recruited at the Zuyderland Medical Center (Sittard, The Netherlands), Rehabilitation \& MS-Center (Overpelt, Belgium) and Biomedical Research Institute (Diepenbeek, Belgium). This study was approved by the institutes' ethics committees (METC14-T-96, 15.143/neuro15.12) and all study subjects gave written informed consent. All samples were stored in the University Biobank Limburg. Further clinical details and numbers of MS patients and HC are provided in Table 3.1. $9 \mathrm{HC}$ and 7 untreated RRMS patients were used for the flow cytometric analysis of costimulatory molecules. Flow cytometric analysis of apoptosis and survival marker (BAFFR) was done on 12 and $14 \mathrm{HC}$, respectively, and 6 untreated RRMS patients. The frequency of Breg was determined via flow cytometry using 5 untreated RRMS patients. Flow cytometric analysis of Breg was done on 5 untreated RRMS patients. In every analysis, HC were matched to MS patients as closely as possible with regard to age and sex.

In order to determine the migration capacity of DN, naive and memory $B$ cells, $\mathrm{CD} 19^{+} \mathrm{CD} 27^{-}$and $\mathrm{CD} 19^{+} \mathrm{CD} 27^{+} \mathrm{B}$ cells of 8 untreated RRMS patients were used in the chemotaxis assay.

Table 3.1 Characteristics of study subjects

\begin{tabular}{|c|c|c|c|c|c|}
\hline Group & $\mathbf{N}$ & Age $^{1}$ & $\begin{array}{c}\text { Female } \\
\% \\
(F: M)^{2}\end{array}$ & EDSS $^{3}$ & $\begin{array}{l}\text { Previous } \\
\text { treatment }\end{array}$ \\
\hline $\begin{array}{l}\text { Costimulatory } \\
\text { molecules } \\
\text { RC } \\
\text { RRMS UT }\end{array}$ & $\begin{array}{l}9 \\
7\end{array}$ & $\begin{array}{l}37 \pm 12 \\
45 \pm 11\end{array}$ & $\begin{array}{l}55(5 / 4) \\
57(4 / 3)\end{array}$ & $\begin{array}{l}\text { N.A. } \\
2.8 \pm 1.8\end{array}$ & $\begin{array}{l}\text { N.A. } \\
\text { UT: } 6 \text {, IFN: } 1\end{array}$ \\
\hline $\begin{array}{l}\text { Apoptosis } \\
\text { HC } \\
\text { RRMS UT }\end{array}$ & $\begin{array}{l}12 \\
6\end{array}$ & $\begin{array}{l}34 \pm 11 \\
45 \pm 8\end{array}$ & $\begin{array}{l}67(8 / 4) \\
66(4 / 2)\end{array}$ & $\begin{array}{l}\text { N.A. } \\
2.8 \pm 1.9\end{array}$ & $\begin{array}{l}\text { N.A. } \\
\text { UT: } 4 \text {, IFN: } 2\end{array}$ \\
\hline $\begin{array}{l}\text { BAFFR } \\
\text { HC } \\
\text { RRMS UT }\end{array}$ & $\begin{array}{l}14 \\
6\end{array}$ & $\begin{array}{l}33 \pm 11 \\
45 \pm 8\end{array}$ & $\begin{array}{l}71(10 / 4) \\
66(4 / 2)\end{array}$ & $\begin{array}{l}\text { N.A } \\
2.8 \pm 1.9\end{array}$ & $\begin{array}{l}\text { N.A. } \\
\text { UT: } 4 \text {, IFN: } 2\end{array}$ \\
\hline$\frac{\text { Breg frequency }}{\text { RRMS UT }}$ & 5 & $38 \pm 13$ & $80(4 / 1)$ & $2 \pm 0.8$ & UT: 5 \\
\hline
\end{tabular}


${ }^{1}$ mean age in years; ${ }^{2}$ number of female (F) and male (M) individuals; ${ }^{3}$ mean Expanded Disability Status Scale (EDSS). IFN = interferon- $\beta$, RRMS = relapsing-remitting multiple sclerosis, $\mathrm{HC}=$ healthy control, N.A. = not applicable, UT $=$ untreated.

\subsubsection{Cell isolation}

Peripheral blood mononuclear cells (PBMC) were isolated from whole blood by Ficoll density gradient centrifugation (Lympholyte, Cedarlane Laboratories, Uden, The Netherlands). B cells were purified from freshly isolated PBMC by negative magnetic selection (STEMCELL Technologies SARL, Grenoble, France). Purity of B cells was routinely $\geq 98.4 \%$.

\subsubsection{In vitro B cell cultures}

DMF and MMF were purchased from Sigma-Aldrich and dissolved in DMSO to make a stock solution of $100 \mathrm{mM}$ and $150 \mathrm{mM}$, respectively. B cells were cultured in 96well round-bottom plates (Greiner Bio-One B.V., Alphen aan den Rijn, The Netherlands) at $3 \times 10^{5}$ cells in RPMI 1640 (Lonza, Basel, Switzerland) with 10\% fetal bovine serum (FBS, Life Technologies, Gent, Belgium), $50 \mathrm{U} / \mathrm{ml}$ penicillin, 50 $\mathrm{mg} / \mathrm{ml}$ streptomycin (Invitrogen, Carlsbad, CA), $0.1 \mathrm{mM}$ nonessential amino acids and $1 \mathrm{mM}$ sodium pyruvate (Sigma-Aldrich).

For analysis of B cell apoptosis, expression of survival marker, costimulatory and antigen presentation molecules, B cells were purified from freshly isolated PBMC and treated during $24 \mathrm{~h}$ with DMF $(10 \mu \mathrm{M}, 16.6 \mu \mathrm{M}, 25 \mu \mathrm{M}$ or $50 \mu \mathrm{M})$ or MMF (50 $\mu \mathrm{M}$ or $100 \mu \mathrm{M}$ ) or were left untreated (DMSO). After $1 \mathrm{~h}$ of treatment, B cells were stimulated with $2 \mu \mathrm{g} / \mathrm{ml} \mathrm{CpG2006}$ (ODN2006, Invivogen, Toulouse, France) for the following $23 \mathrm{~h}$.

When measuring the frequency of $\mathrm{CD} 24^{\text {high }} \mathrm{CD} 38^{\text {high }}$ Breg and TNF- $\mathrm{a}^{+}$proinflammatory $B$ cells, purified $B$ cells were treated during $48 \mathrm{~h}$ with $10 \mu \mathrm{M} D \mathrm{DF}$, $50 \mu \mathrm{M}$ MMF or were left untreated (DMSO) in the presence of $1 \mu \mathrm{g} / \mathrm{ml}$ soluble CD40 ligand together with $2 \mu \mathrm{g} / \mathrm{ml} \mathrm{CpG2006}$ for $48 \mathrm{~h}$. For intracellular flow cytometry, B cells were restimulated during the last $4 \mathrm{~h}$ with PMA ( $50 \mathrm{ng} / \mathrm{ml})$ and calcium ionomycin ( $1 \mu \mathrm{g} / \mathrm{ml}$, both Sigma-Aldrich) in the presence of GolgiStop (BD Biosciences, Erembodegem, Belgium). Fixation and permeabilization were done using Cytofix/Cytoperm (BD Biosciences). 


\subsubsection{Flow cytometry}

Apoptosis and BAFFR expression of B cells was measured by flow cytometry using the following anti-human antibodies: CD19 PE-Cy7, CD268 APC-Cy7 (BioLegend, Antwerp, Belgium), CD27 APC, IgG PE, IgD PE-CF594 (BD Biosciences), annexin $\checkmark$ FITC and 7-AAD (eBioscience, San Diego, USA). B cell expression of costimulatory and antigen presentation molecules was assessed using anti-human CD19 PerCP-Cy5.5, CD27 APC, CD80 PE, CD86 PE-CF594, HLA-DR/DP/DQ FITC (all from BD Biosciences) and CD40 PE-Cy7 (eBioscience).

When analysing the frequency of Breg and pro-inflammatory B cells, the following anti-human antibodies were used: CD19 BV421, CD24 PE-Cy7, IL-10 Brilliant Violet 711 (all from BD Biosciences), CD38 APC-ef780, Fixable Viability Dyeefluor506 (all from eBioscience) and TNF-a PerCP-Cy5.5 (BioLegend). All flow cytometry was performed on a FACSAria II or LSRFortessa flow cytometer and analysed using FACSDiva software (all from BD Biosciences).

\subsubsection{Statistics}

Statistical analyses were performed using Graphpad Prism 6. Wilcoxon matchedpairs signed rank test was used to determine $p$ values between two groups. When comparing multiple groups, one-way ANOVA (Kruskal-Wallis) was used. A p value less than 0.05 was considered statistically significant. 


\subsection{Results}

\subsubsection{DMF but not MMF induces $B$ cell apoptosis in a direct and concentration dependent manner}

Previously, in vitro studies showed that DMF induced T cell apoptosis with a preferential effect on memory T cells (301). Since DMF treatment decreased the percentage of memory B cells while increasing naive B cells (Chapter 2), we investigated whether DMF induced apoptosis of B cells and whether naive B cells showed a lower vulnerability to DMF-induced apoptosis. Here, the direct effect of DMF and MMF on B cell apoptosis (gating strategy depicted in figure 3.1(a)) was investigated. One of the earlier features of apoptosis is the translocation of membrane phospatidylserine (PS) from the inner part of the plasma membrane to the outer part. Annexin V is a dye with a high affinity for PS. In a later stage of apoptosis, membrane integrity is lost, resulting in late apoptotic or necrotic cells. 7-AAD is a vital dye that is excluded from viable cells with intact membranes, while dead and damaged cells are permeable for 7-AAD. 7-AAD is used in combination with annexin $V$ to determine viable cells (Annexin $V^{-} 7-A A D^{-}$), apoptotic cells (Annexin $\mathrm{V}^{+} 7-\mathrm{AAD}^{-}$), late apoptotic cells (Annexin $\mathrm{V}^{+} 7-\mathrm{AAD}^{+}$) and necrotic cells (Annexin $\left.V^{-} 7-A^{+}\right)(320)$.

In $\mathrm{HC}, \mathrm{DMF}$ induced B cell apoptosis at $25 \mu \mathrm{M}(27 \% \pm 11, \mathrm{p}<0.05)$ and $50 \mu \mathrm{M}$ $(48.6 \% \pm 18, \mathrm{p}<0.001)$ compared to baseline $(11.9 \% \pm 6$, Figure $3.1(\mathrm{~b}-\mathrm{c}))$. In untreated MS patients, apoptosis was induced with $50 \mu \mathrm{M}$ DMF $(56.2 \% \pm 14$, p < $0.01)$ compared to baseline $(15.2 \% \pm 5)$ although late $B$ cell apoptosis was already induced at $25 \mu \mathrm{M}(17.7 \% \pm 7, \mathrm{p}<0.05)$ compared to baseline $(7.4 \% \pm$ 2, Figure 3.1(d)). In HC, late apoptosis was only induced at $50 \mu \mathrm{M}$ DMF $(20.4 \%$ $\pm 14, p<0.001)$ compared to baseline $(4.7 \% \pm 3$, Figure $3.1(d))$. Thus, B cells of untreated RRMS patients were already in a late stage of the apoptotic process at a lower DMF concentration when compared to B cells from HC. This suggested that $B$ cells from untreated RRMS patients were more vulnerable to induction of apoptosis by DMF. Furthermore, no significant apoptosis or late apoptosis could be induced at lower concentrations of DMF. Additionally, no effect of DMF could be detected on necrosis of B cells (Figure 3.1(e)). 
In contrast to DMF, MMF treatment did not induce B cell apoptosis (Figure 3.2(ac)) or B cell necrosis (Figure 3.2(d)) and no difference was detected between memory and naive B cells (data not shown). Even at a higher concentration of MMF $(100 \mu \mathrm{M})$, no effect was observed on B cell apoptosis (data not shown). In summary, DMF induced concentration-dependent apoptosis of B cells from $\mathrm{HC}$ and MS patients with B cells of MS patients appearing to be more vulnerable.

(a)

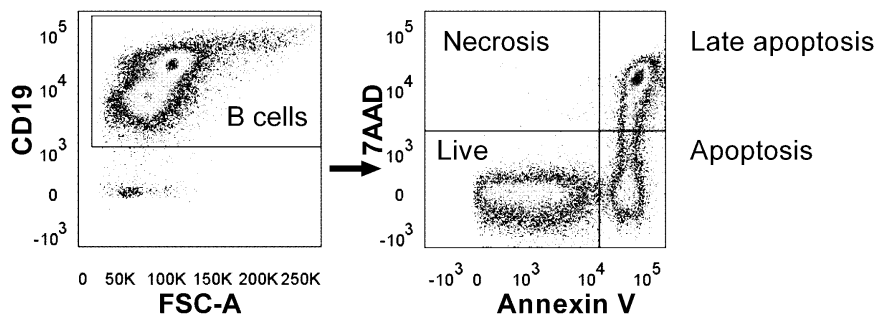

(b)

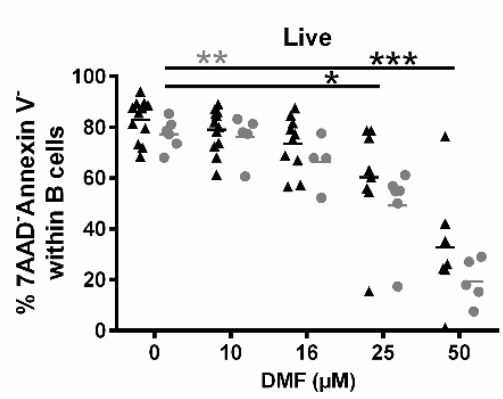

(c)

(d)
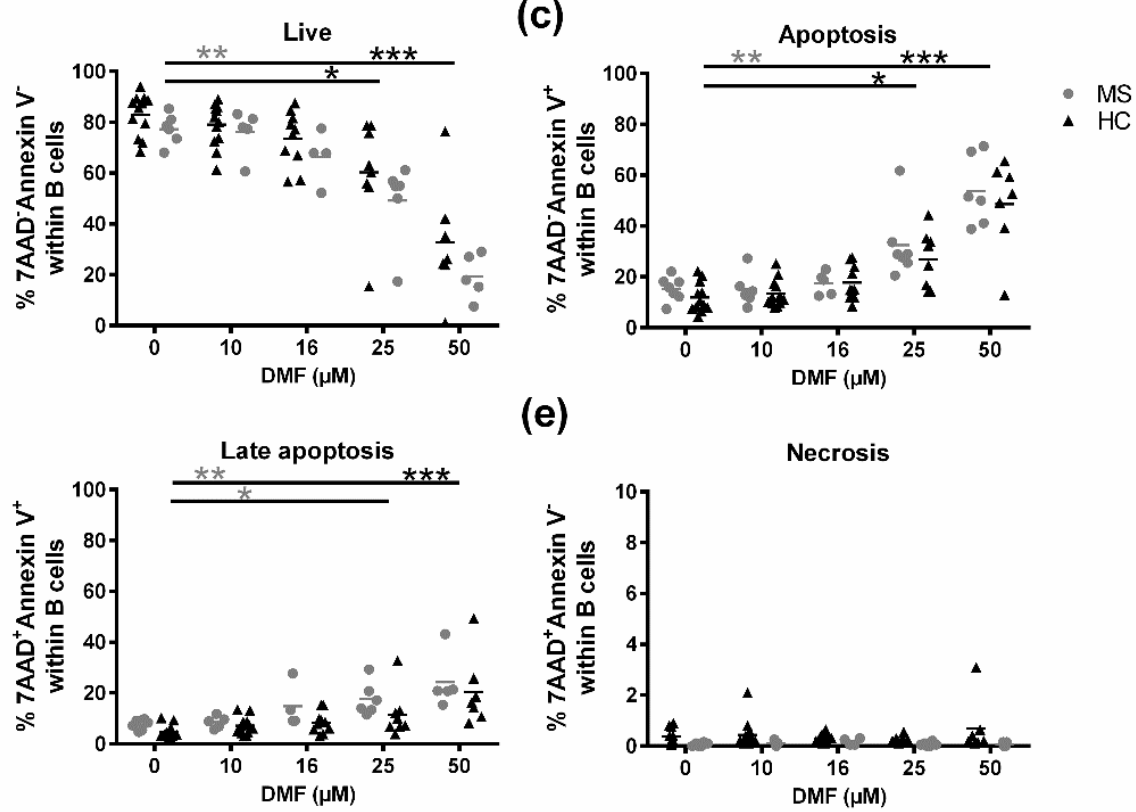

(e)

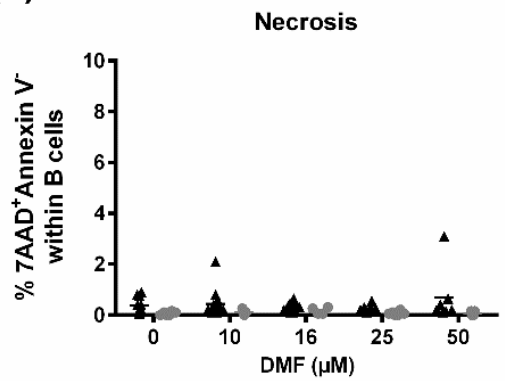

Figure 3.1 DMF induces apoptosis of $B$ cells in a concentration dependent manner. Purified $\mathrm{B}$ cells of $\mathrm{HC}$ and untreated RRMS patients were treated in vitro with the following concentrations of DMF: $10 \mu \mathrm{M}(\mathrm{HC}: \mathrm{n}=12, \mathrm{MS}: \mathrm{n}=5), 16 \mu \mathrm{M}(\mathrm{HC}: \mathrm{n}=10, \mathrm{MS}: \mathrm{n}$ $=4), 25 \mu \mathrm{M}(\mathrm{HC}: \mathrm{n}=8, \mathrm{MS}: \mathrm{n}=6)$ and $50 \mu \mathrm{M}(\mathrm{HC}: \mathrm{n}=7, \mathrm{MS}: \mathrm{n}=5$ ) or left untreated (HC: $n=12, M S: n=6$ ). (a) A representative analysis of apoptosis of $B$ cells is shown. Live (Annexin $\left.\mathrm{V}^{-} 7-A A D^{-}\right)$, apoptotic (Annexin $\mathrm{V}^{+} 7-A A D^{-}$), late apoptotic (Annexin $\mathrm{V}^{+} 7-\mathrm{AAD}^{+}$) and 
necrotic (Annexin $V^{-7}-A A D^{+}$) cells were gated from the $B$ cell population. (b) Frequency of live $B$ cells (Annexin $\left.V^{-} 7-A A D^{-}\right)$, (c) apoptotic $B$ cells (Annexin $V^{+} 7-A A D^{-}$), (d) late apoptotic $B$ cells (Annexin $V^{+} 7-A A D^{+}$) and (e) necrotic $B$ cells (Annexin $V^{-7}-A A D^{+}$) of $H C$ and $M S$ patients. A Kruskal-Wallis one-way ANOVA was used to compare the different groups. * $\mathrm{p}<$ $0.05, * * \mathrm{p}<0.01, * * * \mathrm{p}<0.001 . \mathrm{HC}=$ healthy control, MS $=$ multiple sclerosis, $\mathrm{DMF}=$ dimethyl fumarate.

(a)

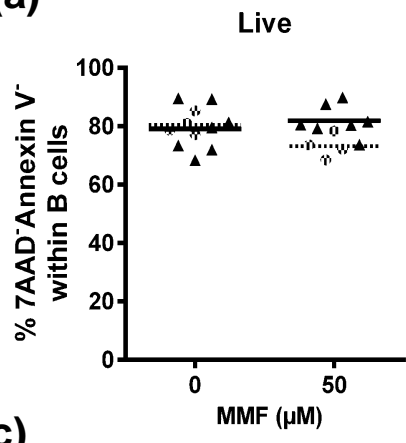

(c) Late apoptosis

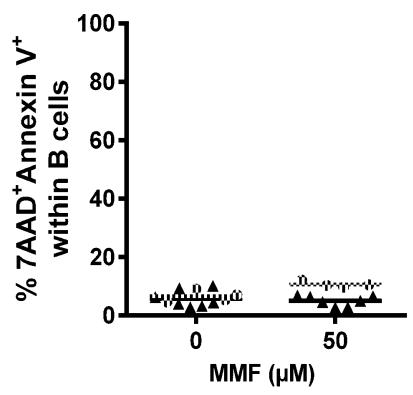

(b)

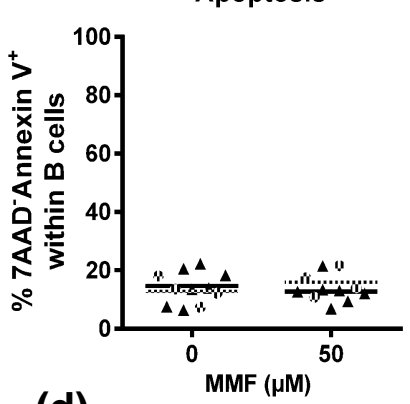

(d)

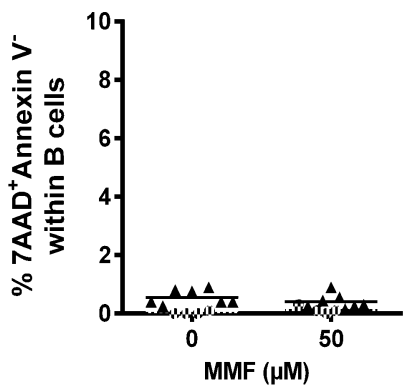

Figure 3.2 MMF has no effect on apoptosis of $B$ cells. Purified $B$ cells of $H C$ and untreated RRMS patients were treated in vitro with $50 \mu \mathrm{M}$ MMF or left untreated (HC: $n=$ 7, MS: $n=4$ ). (a) Frequency of live $B$ cells (Annexin $V^{-7-A A D}{ }^{-}$), (b) apoptotic $B$ cells (Annexin $\left.V^{+} 7-A A D^{-}\right),(c)$ late apoptotic $B$ cells (Annexin $V^{+} 7-A A D^{+}$) and (d) necrotic $B$ cells (Annexin $\mathrm{V}-7-\mathrm{AAD}^{+}$) of $\mathrm{HC}$ and MS patients. Wilcoxon matched-pairs signed rank test was used. $\mathrm{HC}=$ healthy control, $\mathrm{MS}=$ multiple sclerosis, MMF = monomethyl fumarate. 


\subsubsection{DMF directly reduces BAFFR expression on B cells}

Survival and maturation of $B$ cells depend on the interaction of the $B$ cell survival marker BAFF with BAFFR (321). Since DMF induced apoptosis of B cells, it was investigated whether DMF could also affect the survival of $B$ cells through modulation of the BAFFR expression levels. DMF, but not MMF, directly decreased BAFFR expression on B cells in vitro, already at $10 \mu \mathrm{M}$ (HC: $\mathrm{p}<0.05$; MS: $\mathrm{p}=$ 0.0625 ) with stronger effects using higher DMF concentrations ( $p<0.01$ ) when compared to baseline (Figure 3.3(a)). An increased baseline BAFFR expression level was observed on MS B cells compared to $\mathrm{HC}$ ( $\mathrm{p}<0.0001$, Figure 3.3(b)). In our previous study (Chapter 2), no effect of DMF treatment was detected on the expression of BAFFR on B cells or on BAFF plasma levels in RRMS patients after $12 \mathrm{~m}$ of DMF treatment. This suggests that DMF treatment has an acute direct effect on B cell survival by decreasing BAFFR expression, which cannot be detected ex vivo due to long term treatment and a shift in B cell subtypes. 
(a)
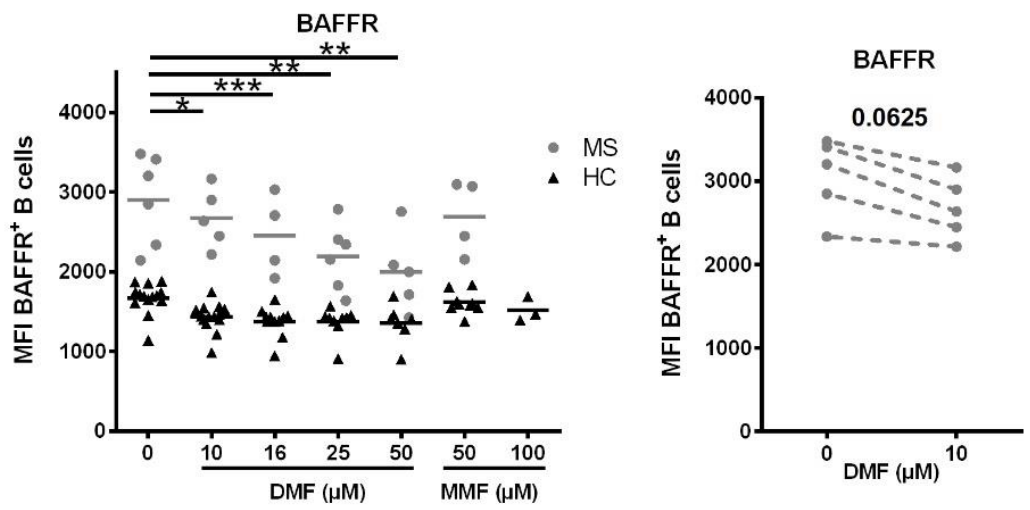

(b)

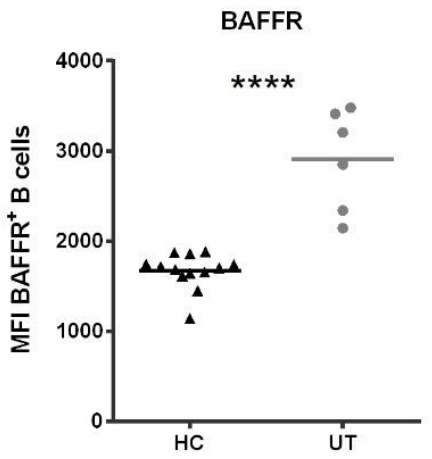

Figure 3.3 DMF downregulates the expression of survival marker BAFFR on B cells. (A) The expression of BAFFR on B cells of $H C$ and untreated RRMS patients. Purified $B$ cells of $H C$ and untreated RRMS patients were treated in vitro with the following concentrations of DMF: $10 \mu M(H C: n=14, M S: n=5), 16 \mu M(H C: n=11, M S: n=4), 25$ $\mu M(H C: n=9, M S: n=6)$ and $50 \mu M(H C: n=7, M S: n=5)$, or with MMF: $50 \mu M(H C: n$ $=9, M S: n=4), 100 \mu M(H C: n=3)$ or were left untreated (HC: $n=14$ and MS: $n=5)$. (B) The expression of BAFFR on B cells of HC $(n=13)$ and of untreated RRMS patients ( $n$ $=6$ ). A Kruskal-Wallis one-way ANOVA was used to compare the different groups and a Wilcoxon matched-pairs signed rank test was used to determine $p$ values between two groups. $* \mathrm{p}<0.05, * * \mathrm{p}<0.01, * * * \mathrm{p}<0.001, * * * * \mathrm{p}<0.0001 . \mathrm{HC}=$ healthy control, $\mathrm{MS}=$ multiple sclerosis, $\mathrm{DMF}=$ dimethyl fumarate, $\mathrm{MMF}=$ monomethyl fumarate, $\mathrm{MFI}=$ mean fluorescence intensity, BAFFR $=B$ cell activating factor receptor.

\subsubsection{DMF directly reduces the expression of antigen presentation molecules and costimulatory molecule CD40 on B cells.}

We previously demonstrated the potential of MS B cells to induce autoreactive T cell responses via antigen presentation and increased costimulatory molecule expression on MS B cells (123). As immunomodulatory treatment abrogated these effects (123), we investigated whether in vitro treatment with DMF directly 
decreased the expression of antigen presentation and costimulatory molecules on $B$ cells. DMF, but not MMF, directly decreased B cell expression of HLA-DR/DP/DQ and CD40 in $\mathrm{HC}$ ( $\mathrm{p}=0.0234$ and $\mathrm{p}=0.0078$, respectively) and untreated MS patients $(p=0.0313$ and $p=0.0313$, respectively, Figure $3.4(a-b))$.
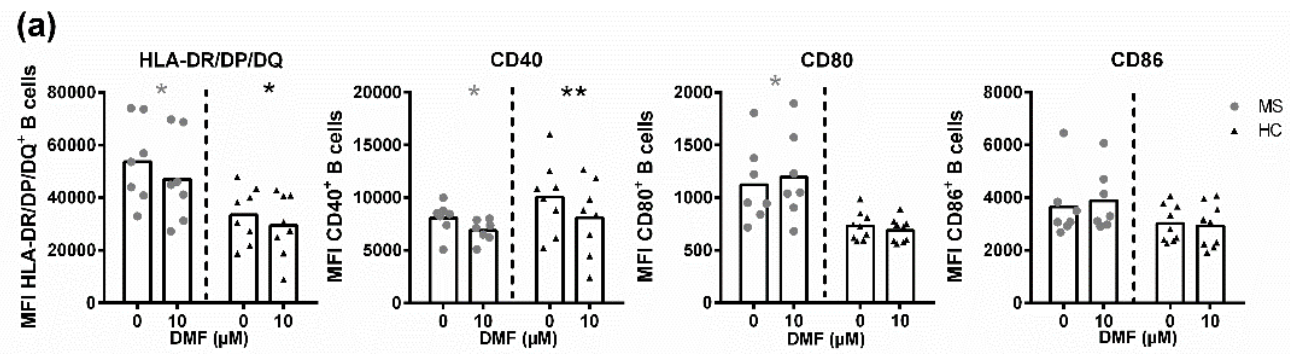

(b)
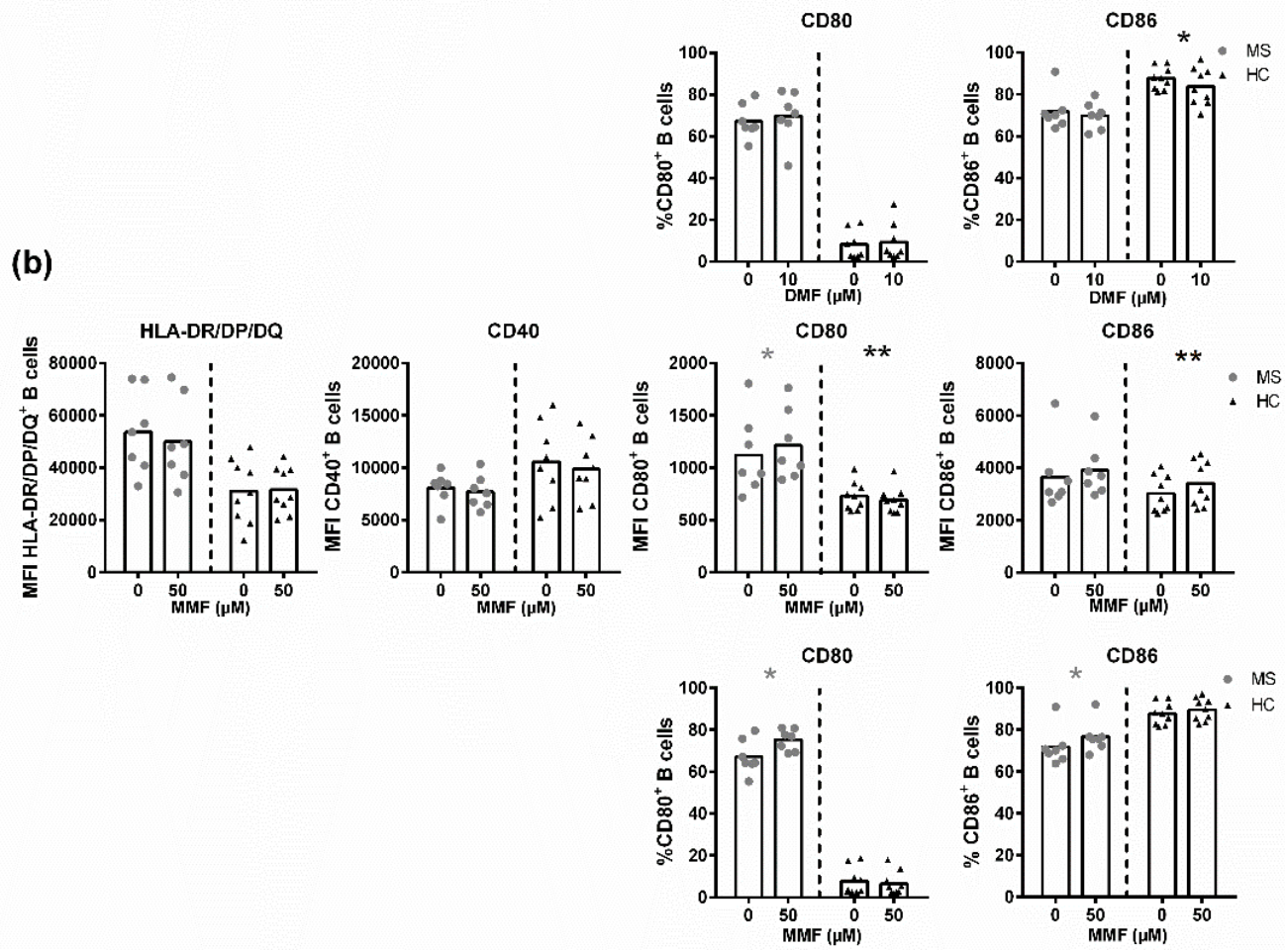

Figure 3.4 DMF downregulates the expression of antigen presentation molecules and costimulatory molecule CD40 on B cells.

(a) Purified B cells of $\mathrm{HC}$ and untreated RRMS patients were treated in vitro with $10 \mu \mathrm{M}$ DMF or were left untreated. Expression (MFI) of HLA-DR/DP/DQ (HC: $n=8, M S: n=7$ ), CD40 (HC: $n=8, M S:=7)$, CD80 (HC: $n=9, M S: n=7)$ and CD86 (HC: $n=9, M S: n=7)$ on 
B cells and $\% \mathrm{CD}^{+} 0^{+}(\mathrm{HC}: \mathrm{n}=8, \mathrm{MS}: \mathrm{n}=7)$ and $\% \mathrm{CD}^{+}(\mathrm{HC}: \mathrm{n}=9, \mathrm{MS}: \mathrm{n}=7)$ B cells is depicted for $\mathrm{HC}$ and MS patients. (b) Purified $\mathrm{B}$ cells of $\mathrm{HC}$ and untreated RRMS patients were treated in vitro with $50 \mu \mathrm{M}$ MMF or were left untreated. Expression (MFI) of HLADR/DP/DQ (HC: $n=9, M S: n=7$ ), CD40 (HC: $n=8, M S:=7)$, CD80 (HC: $n=9, M S: n=$ 7) and CD86 (HC: $n=9, M S: n=7)$ on $B$ cells and $\% \mathrm{CD}^{+} 0^{+}(\mathrm{HC}: \mathrm{n}=9, \mathrm{MS}: \mathrm{n}=7)$ and $\% \mathrm{CD}^{+} 6^{+}$(HC: $n=9$, MS: $n=7$ ) B cells is depicted for HC and MS patients. A Wilcoxon matched-pairs signed rank test was used to determine $\mathrm{p}$ values between two groups. $* \mathrm{p}<$ $0.05, * * \mathrm{p}<0.01 . \mathrm{HC}=$ healthy control, MS = multiple sclerosis, DMF = dimethyl fumarate, $\mathrm{MMF}=$ monomethyl fumarate, MFI = mean fluorescence intensity, human leucocyte antigen -DR (HLA-DR).

In contrast, DMF increased CD80 expression on B cells of untreated MS patients ( $p=0.0469$, Figure 3.4(a)), while no effect was detected in HC or on CD86 expression. In addition, untreated MS patients showed a higher baseline expression ( $p=0.0164)$ and percentage of CD80 ( $p=0.0003)$ on $B$ cells compared to $\mathrm{HC}$. DMF had no effect on the percentage of $\mathrm{CD}^{+} 0^{+} \mathrm{B}$ cells while it decreased the percentage of $\mathrm{CD} 6^{+} \mathrm{B}$ cells in HC. Furthermore, MMF decreased the expression of CD80 and increased the expression of CD86 in HC, while an increase in the expression CD80 and no effect on the expression of CD86 was detected on $B$ cells of untreated MS patients. In addition, MMF increased the percentage of $\mathrm{CD}^{+} 0^{+}$and $\mathrm{CD} 86^{+} \mathrm{B}$ cells of untreated MS patients while no effect was detected in $\mathrm{HC}$. In conclusion, DMF, but not MMF, decreases the expression of antigen presentation molecule HLA-DR/DP/DQ and costimulatory molecule CD40 on B cells of RRMS patients and HC, while both DMF and MMF increase the expression of CD80 on B cells of RRMS patients.

\subsubsection{DMF tends to directly affect frequencies of pro- and anti- inflammatory $B$ cells}

To examine the effect of DMF on the frequency of Breg and pro-inflammatory cytokine-expressing B cells (gating strategy depicted in figure 3.5(a)), purified B cells from 5 untreated RRMS patients were treated in vitro with DMF or MMF. Although it is not significant, a trend towards an increased frequency of Breg was induced by DMF ( $p=0.06$ ), while MMF had no effect (Figure 3.5(b)). However, MMF but not DMF, decreased the frequency of TNF- $a^{+} B$ cells, although not significantly (Figure 3.5(b) $\mathrm{p}=0.06$ ). DMF and MMF showed no effect on the frequency of $\mathrm{IL}-10^{+} \mathrm{B}$ cells (Figure $3.5(\mathrm{~b})$ ). Summarized, these results indicate 
that DMF could directly increase anti-inflammatory B cell frequencies while MMF could decrease pro-inflammatory B cell frequencies.

(a)

(b)
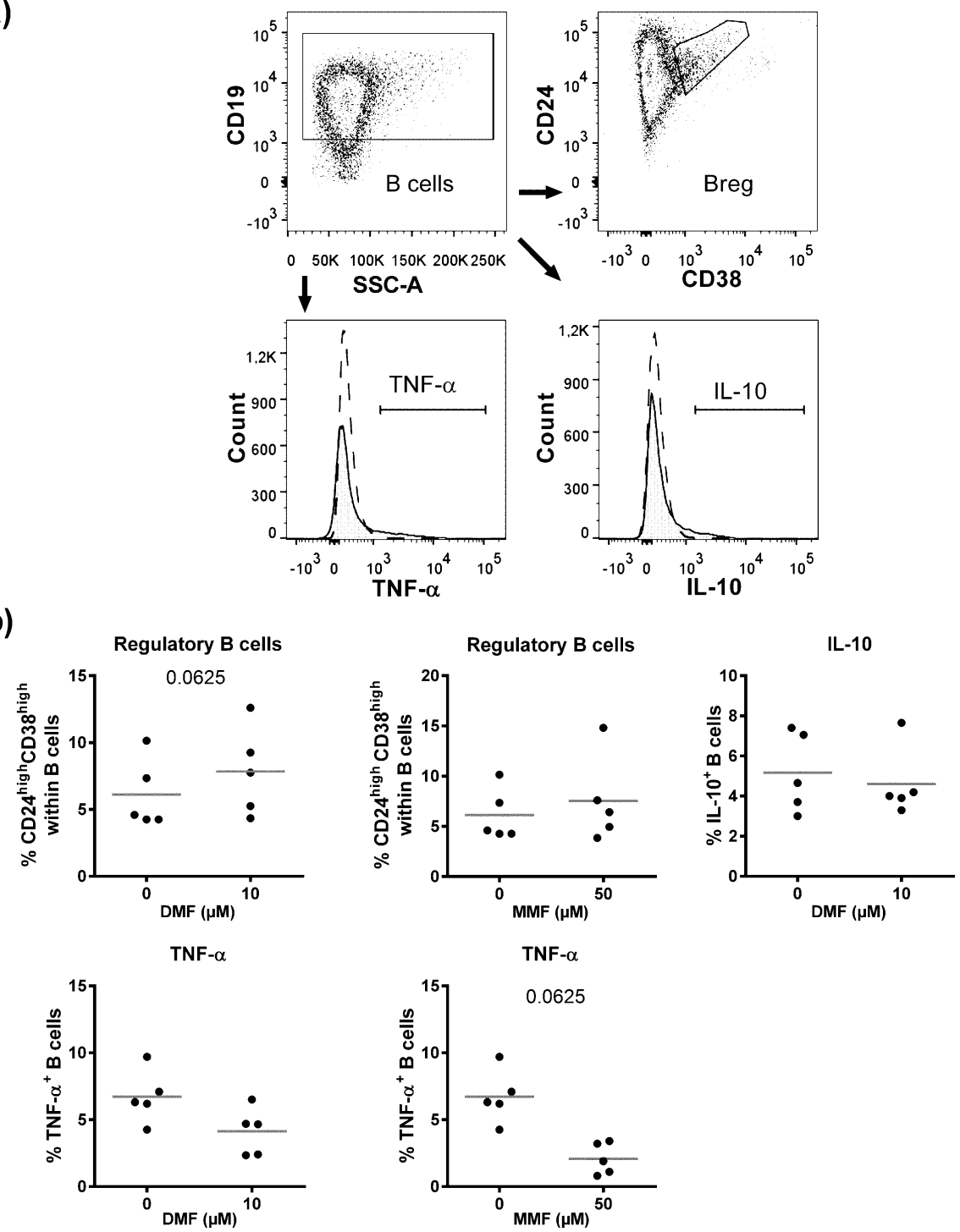

Figure 3.5 A trend towards an increase in Breg is induced by DMF while MMF tends to decrease TNF-a $a^{+}$cells in a direct manner. Purified $B$ cells of untreated RRMS patients ( $n=5$ ) were treated in vitro with $10 \mu \mathrm{M} D M F, 50 \mu M$ MMF or were left untreated. (a) A representative analysis of Breg and $B$ cell cytokines is shown. Breg $\left(\mathrm{CD} 24^{\text {high }} \mathrm{CD} 38^{\text {high }}\right), \mathrm{IL}-10^{+}$and TNF- $\mathrm{a}^{+}$cells were gated from the $\mathrm{B}$ cell population based on unstimulated controls (dashed lines). (b) Frequencies of Breg (CD24 $\left.4^{\text {high }} \mathrm{CD} 38^{\text {high }}\right), \mathrm{IL}-10^{+} \mathrm{B}$ cells and TNF- $\mathrm{a}^{+} \mathrm{B}$ cells are depicted. Wilcoxon matched-pairs signed rank test was used to 
determine $\mathrm{p}$ values. $\mathrm{DMF}=$ dimethyl fumarate, $\mathrm{MMF}=$ monomethyl fumarate, $\mathrm{TNF}=$ tumor necrosis factor. 


\subsection{Discussion}

DMF is an important oral treatment approved for RRMS patients, with more than 4 years of "real-world" usage, although its mechanism(s) of action is not completely understood. Several studies have shown that DMF treatment has an immune modulating effect by inducing changes in the distribution of $\mathrm{T}$ and $\mathrm{B}$ cell subtypes $(199,295,300,304,316,317)$. Nonetheless, little is known about the effect of DMF on functional markers of B cells. In this study, we analyzed the direct effect of DMF and MMF on B cells of HC and RRMS patients. We showed that DMF induced direct and concentration-dependent apoptosis of $\mathrm{B}$ cells and decreased survival marker BAFFR, B cell expression of the costimulatory molecule CD40 and antigen presentation MHCII molecule. Furthermore, DMF tended to directly increase Breg frequencies while MMF tended to decrease the frequency of TNF- $\mathrm{a}^{+}$ $B$ cells.

Gillard et al. demonstrated that DMF and MMF are active between 5.5 and $50 \mu \mathrm{M}$ and 50 and $150 \mu \mathrm{M}$, respectively (251). Therefore, DMF was used here at different concentrations between $10 \mu \mathrm{M}$ and $50 \mu \mathrm{M}$, while $50 \mu \mathrm{M}$ of MMF was used (251).

In a phase III clinical trial, about 6-9\% of DMF-treated MS patients experienced grade 3 lymphopenia (286). Since DMF treatment of RRMS patients resulted in a drop in the absolute counts of almost all immune cell subtypes and reductions in the frequency of $B$ cell subtypes, we investigated induction of apoptosis as one of the underlying mechanisms (316). DMF induced concentration-dependent apoptosis of polyclonally stimulated $\mathrm{B}$ cells from $\mathrm{HC}$ and untreated RRMS patients starting from a concentration of $25 \mu \mathrm{M}$ DMF, with B cells of MS patients appearing to be more vulnerable. As MMF did not have an effect on B cell apoptosis, we propose that DMF is responsible for the full effect on the reduction of $B$ cell numbers. Although DMF could not be detected in serum of $\mathrm{HC}$, a degree of systemic penetrance of DMF has been reported with DMF-glutathione conjugates found in the urine of DMF-treated psoriasis patients (247). Furthermore, Litjens et al. demonstrated that DMF could be taken up by lymphocytes and monocytes (244).

In Chapter 2, we showed that DMF treatment decreased the percentage of memory B cells while increasing naive and transitional B cells after 12 months of treatment (316). 
Here, we demonstrated that DMF induced apoptosis of B cells without having a preferential effect on memory or naive $B$ cells. Thus, the increase in the frequency of naive $B$ cells observed in previous studies is not due to a lower vulnerability of naive $B$ cells to DMF-induced apoptosis. Indirect effects, such as the interplay between immune cells or the output of new $B$ cells could be responsible for the effects observed in Chapter 2. However, it is still possible that transitional B cells are less sensitive to DMF-induced apoptosis than memory or naive B cells. For instance, Li et al. detected a preferential effect of in vitro DMF induced apoptosis on mature B cells compared to transitional B cells of HC (295).

DMF directly reduced B cell expression of the survival marker BAFFR. However, follow-up of DMF treated RRMS patients (Chapter 2) showed that BAFF serum levels and $B$ cell BAFFR expression were unchanged after $12 \mathrm{~m}$ of DMF treatment (316). The latter effect could be due to increased transitional and naive $B$ cells after DMF treatment. BAFF has been described to play a dual role in MS pathology including both pathological effects (322) and positive effects on treatment outcome and detrimental effects of BAFF antagonists (323). Interestingly, BAFFR expression was increased on $B$ cells of untreated MS patients compared to HC. An increased BAFFR mRNA level was previously demonstrated in MS patients (324). BAFF is important for maintaining antibody-producing plasma cells and the direct effect of DMF on BAFFR expression could be beneficial for MS pathogenesis.

Due to the fact that the exact concentration of DMF in the circulation or in immune cells of RRMS patients is not known and cannot be determined exactly, it is important to investigate the direct effects of DMF on $B$ cells in low concentrations in vitro. Since Blewett et al. demonstrated that DMF directly interacts with cysteine residues of $\mathrm{T}$ cells in concentrations between 10 and $50 \mu \mathrm{M}$ and that this is mechanistically relevant to the immunosuppressive activity of the drug, and since we have demonstrated that no apoptosis is induced in concentrations below 25 $\mu \mathrm{M}$, we examined whether DMF also exerted a direct effect on functional markers of $B$ cells at a concentration of $10 \mu \mathrm{M}$ (258). In this study, we demonstrated that DMF directly decreased $B$ cell expression of antigen presentation molecules HLA$\mathrm{DR} / \mathrm{DP} / \mathrm{DQ}$ and costimulatory molecule CD40. This could lead to reduced $\mathrm{T}$ cell activation, since B cells are critical antigen presenting cells in MS that can stimulate $T$ cell proliferation to foreign or neuro-antigens (123). 
Previous reports demonstrated increased CD40 ligand on MS T cells and hyperresponsiveness of MS memory B cells to CD40 stimulation $(325,326)$. Therefore, we suggest that the decreased B cell CD40 expression induced by DMF will result in disrupted B cell activation and thus B cell mediated pathology. Furthermore, these results could explain the decrease in memory $T$ cells and reduced $T$ cell proliferation and activation following DMF treatment (300, 305, 308, 309, 327). Differential effects of DMF and MMF on the expression and percentages of CD80 and CD86 were observed on B cells of RRMS patients and HC, which makes it difficult to draw a clear conclusion. This can be due to the low number of patients and $\mathrm{HC}$ used in the study. Furthermore, the differences that are detected are rather small, which could mean that inclusion of more RRMS patients and HC in the study may obliterate the small difference that is detected now. On the other hand, both DMF and MMF increase the expression of CD80 on B cells of RRMS patients. This increase could be the result of a rescue mechanism of $B$ cells. Furthermore, this study confirms that there is an elevated baseline expression of B cells in RRMS patients, as compared to HC (123).

Interestingly, transitional B cells are enriched for Breg, which could have beneficial effects on the MS pathogenesis (86). Using in vitro treatment of B cells with DMF and MMF, we showed that DMF but not MMF increased the frequencies of Breg, although not significantly. These results are particularly intriguing with regard to the role of Breg in the control of autoimmunity. In humans, Breg suppress Th1 and Th17 differentiation while inducing the generation of Treg (328). This suggests that the decreased frequency of $T$ cells expressing Th1 and Th17 cytokines following DMF treatment, as shown in Chapter 2, could be an indirect consequence of an increase in Breg (316). Further, these results demonstrate that the increase in frequency of Breg after DMF treatment as seen in a follow-up study conducted by Lundy et al. could be regulated in a direct manner (311). Furthermore, no difference in IL-10 expressing B cells was detected. This is in agreement with Li et al. and Smith et al. who also did not detect a difference in IL-10 expression in the total number of B cells $(295,310)$. However, Staun-Ram et al. detected an increased percentage of $\mathrm{IL}-10^{+}$regulatory $\mathrm{B}$ cells using different markers associated with Bregs (CD25, CD5, CD1D, CD80, and CD86) in MS patients after $3.5 \mathrm{~m}$ of DMF treatment (329). In addition, we detected a trend towards a decrease in the frequency of TNF- $\mathrm{a}^{+} \mathrm{B}$ cells by MMF, which implies that 
the decrease in the frequency of TNF- $\mathrm{a}^{+} \mathrm{B}$ cells shown in a follow-up study by Smith et al. and Li et al. could be induced in a direct manner $(295,310)$.

In conclusion, DMF induced apoptosis of B cells and decreased the expression of functional B cell markers which are involved in antigen presentation and survival, whereas it increased the frequency of regulatory B cells, which could influence B cell function. 


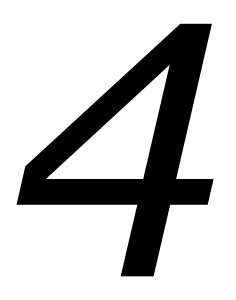

\section{Phenotypical and functional} characterization of IgD ${ }^{-}$CD27double negative (DN) B cells in multiple sclerosis patients

Partly based on:

Phenotypic and Ig Repertoire Analyses Indicate a Common Origin of IgD(-)CD27(-) Double Negative B Cells in Healthy Individuals and Multiple Sclerosis Patients.

Fraussen J, Marquez S, Takata K, Beckers L, Montes Diaz G, Zografou C, Van Wijmeersch B, Villar LM, O'Connor KC, Kleinstein SH, Somers V J Immunol. 2019

Contribution to the paper: flow cytometric analysis of the phenotype of DN B cells from healthy individuals and MS patients 


\section{Phenotypical and functional characterization of IgD'CD27- double negative (DN) B cells in multiple sclerosis patients}

\subsection{Abstract}

Increased frequencies of a specific subtype of B cells, namely IgD-CD27- double negative (DN) B cells with proinflammatory characteristics, have been described in healthy aging and in autoimmune diseases. In this study, we investigated the developmental and migratory phenotype and functional characteristics of DN B cells in untreated MS patients. The expression of the developmental markers and immunoglobulin (Ig) isotypes was measured on DN, IgD-CD27+ class-switched memory (CSM) and IgD ${ }^{+}$CD27- naive $B$ cells of healthy controls ( $\mathrm{HC}, \mathrm{n}=48$ ) and untreated MS patients $(n=96)$. The distribution of several adhesion molecules, chemokine receptors and the T-box transcription factor T-bet was investigated in DN, CSM and naive B cells of HC $(n=25)$ and untreated MS patients $(n=49)$. Using an in vitro chemotaxis assay, migration of peripheral blood CD27- or CD27+ $B$ cells of untreated MS patients $(n=7)$ towards the pro-inflammatory chemokines C-X-C motif ligand (CXCL)-13 and CXCL10 was analyzed. DN B cells from HC and untreated MS patients showed similar phenotypical characteristics. We demonstrated that DN B cells are mature, as indicated by low expression of CD5, CD38 and CD10. The majority of DN B cells was antigen experienced by expressing IgG ( $45 \%)$ or IgA ( 20\%). However, the frequency of IgA $\mathrm{I}^{+}$and $\mathrm{CD}^{+} 5^{+}$cells was lower in DN compared to CSM B cells. T-bet was expressed by $\sim 22 \%$ DN B cells. In addition, DN B cells showed an intermediate expression of adhesion molecules (LFA-1, VLA-4 and ALCAM) and chemokine receptor CXCR3 between naive and CSM B cells. Furthermore, DN B cells of untreated MS patients migrated towards CXCL13 and CXCL10 in vitro and showed a higher migration capacity for CXCL13 compared to naive and non class-switched memory (NCSM, IgD ${ }^{+} \mathrm{CD} 27^{+}$) B cells, similar to CSM B cells. In conclusion, DN B cells are mature, mostly antigen experienced cells that have a high migratory capacity towards chemokines involved in B cell recruitment into the central nervous system (CNS) of MS patients. 


\subsection{Introduction}

Multiple sclerosis (MS) is a chronic inflammatory disease of the central nervous system (CNS). MS is characterized by recruitment of immune cells to the CNS, leading to autoimmune attacks against components of the CNS (myelin, neurons, astrocytes, oligodendrocytes) (1). MS is considered to be a T and B cell mediated disease. B cells are found at sites of tissue injury in the CNS. They are also found in the cerebrospinal fluid (CSF), white matter lesions, gray matter, and in the meninges, where they form lymphoid-like tissue aggregates (330) that associate with proximal tissue damage (331). The $B$ cells that populate distinct compartments of the CNS are clonally related (332) and link to populations in the periphery (91). Furthermore, they are responsible for the production of the oligoclonal Ig bands in the CSF that remain a hallmark of the disease $(333,334)$. The strongest evidence supporting their role comes from B cell-depleting therapy, which demonstrates remarkable efficacy in relapsing-remitting MS (RRMS) and even primary progressive MS (PPMS)(59, 97, 98). These collective findings notwithstanding, the mechanisms underpinning the pathogenic contribution of $B$ cells require further understanding.

During human aging, quantitative and functional changes occur in the innate and adaptive immune system that lead to increased bacterial and viral infections, while vaccine efficacy declines $(145-147,335)$. Advancing age is accompanied by an increase in the levels of inflammatory mediators in the absence of acute infections, which leads to a chronic low-grade inflammation level known as inflamm-aging (145-147). In elderly, the prevalence of autoimmune diseases is increased and aging is linked to autoimmunity $(336,337)$. Interestingly, a proportion of MS patients display characteristics of premature immune aging that is thought to contribute to MS pathogenesis and progression $(144,154)$.

Abnormalities in specific subtypes of the B cell lineage have been increasingly identified in healthy elderly and in autoimmune diseases, including MS (68, 69, $90)$. One such $B$ cell subtype is the age-associated IgD-CD27- double negative (DN) B cell. Much of our current knowledge on DN B cells is based on studies in other autoimmune diseases (systemic lupus erythematosus (SLE) and rheumatoid arthritis (RA)), while studies on the phenotype and function of DN B cells in the pathology of MS are sparse. 
The aim of this study was to characterize the developmental and migratory phenotype and function of DN B cells in untreated MS patients. Our research group recently reported increased frequencies of DN B cells in peripheral blood of a proportion ( $20 \%$ ) of young MS patients ( $<60$ years) compared to young healthy controls (HC). In addition, DN B cells produced pro-inflammatory and cytotoxic cytokines following ex vivo stimulation and their potential to stimulate $T$ cell responses was indicated by expression of the antigen presentation molecule MHC class II and the costimulatory molecules CD80 and CD86. Furthermore, a higher frequency of DN B cells was found in the CSF of MS patients compared to paired peripheral blood (68). Moreover, Palanichamy et al. reported that peripheral blood DN B cells of MS patients are clonally related to CSF DN B cells, which indicates that they could act as important contributors to disease-associated B cell repertoires in MS patients (91).

$C D 21^{\text {low }} B$ cells are another population of age-associated $B$ cells that is linked with the expression of the transcription factor T-box expressed in $\mathrm{T}$ cells ( $\mathrm{T}$-bet). $\mathrm{T}$-bet ( $T$-Box Expressed in T cells) is a transcription factor that is encoded by the $T b \times 21$ gene and was first described as a transcriptional regulator of T helper 1 (Th1) cell differentiation (338). In B cells, T-bet plays an important role during anti-viral immunity, as T-bet $^{+} \mathrm{B}$ cells are elevated during antiviral responses and secrete IgG2a antibodies for clearing pathogens (174). Moreover, it was shown in mice that T-bet expression is required for the production of pathogenic autoantibodies (338). In mice, $T$-bet is a master regulator of the age-associated $B$ cell character and overexpression of $\mathrm{T}$-bet induces acquisition of the age-associated $\mathrm{B}$ cell phenotype (172). We have previously shown that about $20 \%$ of DN B cells could be retracted to the $C D 21^{\text {low }} B$ cell gate and that $D N B$ cells expressed the lowest $C D 21$ levels of all $B$ cell subtypes (68). CD21 low $B$ cells show similarities with ageassociated B cells of aged and autoimmune-prone mice and SLE patients. Furthermore, in SLE patients, IL-21 can drive the differentiation of T-bet ${ }^{+}$ageassociated $B$ cells into auto-antibody-producing plasma cells, which links the expression of T-bet to autoimmunity (339). Since T-bet is involved in autoimmunity and is a master regulator of age-associated B cells in mice, it is interesting to further study if $C D 21^{\text {low }} B$ cells and DN B cells of MS patients express T-bet and if this is also a master regulator of these cells. 
Since we previously identified DN B cells in the CSF of MS patients (68), we measured their expression of adhesion molecules and inflammatory chemokine receptors and the migratory potential of DN $B$ cells. $B$ cells depend on the expression of adhesion molecules (LFA-1, VLA-4 and ALCAM) to migrate through the BBB $(139,140,340)$. Furthermore, in MS patients it has been shown that CNS $B$ cell trafficking is influenced by chemokine receptors CXCR3 and CXCR5 (127, $128,340)$. CSF levels of CXCL13 are increased in active RRMS patients and correlate with the number of total B cells in the CSF and even more specifically with the number of CSM B cells in the CSF (129). In addition, the inflammatory chemokine CXCL10 contributes to lesion development in MS and its concentration is significantly increased in the CSF of MS patients (130).

Altogether, DN B cells may play a relevant role in the immune axis connecting the periphery with the CNS, nonetheless further work is necessary to understand the relevance of DN B cells in MS. In order to study the developmental phenotype of DN B cells, we first performed a detailed phenotypic characterization of DN B cells from HC and untreated MS patients using different markers for B cell subtype classification, maturation, adhesion, migration and T-bet expression. Furthermore, we investigated the migratory capacity and the activation state of DN B cells of MS patients. 


\subsection{Material and methods}

\subsubsection{Study subjects}

MS patients (RRMS, PPMS, SPMS) and HC were recruited at the Zuyderland Medical Center (Sittard, The Netherlands), Rehabilitation \& MS-Center (Pelt, Belgium), Biomedical Research Institute (Diepenbeek, Belgium) and Hospital Universitario Ramón y Cajal (Madrid, Spain). The study was approved by the institutes' ethics committees and all study subjects signed a written informed consent. All samples were stored in the University Biobank Limburg. Clinical and demographic information of included untreated MS patients and $\mathrm{HC}$ are provided in Table 4.1. 


\section{Table 4.1. Characteristics of study subjects}

${ }^{1}$ mean age in years; ${ }^{2}$ number of female (F) and male (M) individuals; ${ }^{3}$ mean expanded disability status scale (EDSS); ${ }^{4}$ mean disease duration in years; UT = untreated, IFN = interferon- $\beta, \mathrm{GA}=$ glatiramer acetate, $\mathrm{TF}=$ teriflunomide; $\mathrm{RR}=$ relapsing-remitting, $\mathrm{SP}=$ secondary progressive; $\mathrm{PP}=$ primary progressive; $\mathrm{HC}=$ healthy control; N.A. = not applicable.

\begin{tabular}{|c|c|c|c|c|c|c|c|}
\hline Group & $\mathbf{N}$ & $\mathrm{Age}^{1}$ & $\begin{array}{l}\text { Female } \\
\% \\
(\mathrm{~F}: M)^{2}\end{array}$ & EDSS $^{3}$ & $\begin{array}{l}\text { Previous } \\
\text { treatment }\end{array}$ & $\begin{array}{l}\text { MS } \\
\text { type }\end{array}$ & $\begin{array}{l}\text { Disease } \\
\text { duration } 4\end{array}$ \\
\hline 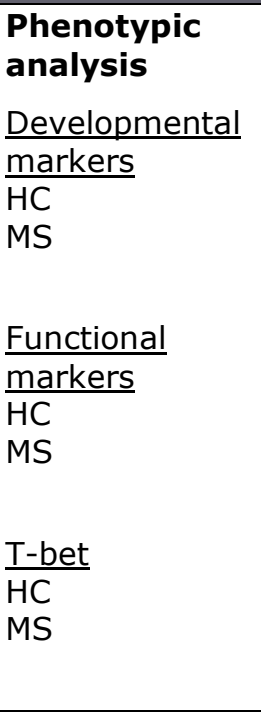 & $\begin{array}{l}25 \\
49\end{array}$ & $\begin{array}{l}46 \pm 16 \\
44 \pm 13 \\
\\
42 \pm 11 \\
45 \pm 10 \\
\\
41 \pm 10 \\
45 \pm 10\end{array}$ & $\begin{array}{l}69(33: 15) \\
73(70: 26) \\
72(18: 7) \\
74(36: 13) \\
71(17: 7) \\
75(35: 12)\end{array}$ & $\begin{array}{l}\text { N.A. } \\
3.4 \pm 2.2 \\
\text { N.A. } \\
3.7 \pm 2.1 \\
\text { N.A. } \\
3.7 \pm 2.1\end{array}$ & $\begin{array}{l}\text { N.A. } \\
\text { UT: 76, IFN: } \\
\text { 9, GA: 2, } \\
\text { TF: } 1 \\
\text { N.A. } \\
\text { UT: 38, IFN: } \\
\text { 5, GA: 2, } \\
\text { TF: } 1 \\
\text { N.A. } \\
\text { UT: } 36, \text { IFN: } \\
\text { 5, GA: 2, } \\
\text { TF: } 1\end{array}$ & $\begin{array}{l}\text { N.A. } \\
\text { RR: } 63 \\
\text { PP: } 13 \\
\text { SP: } 20 \\
\\
\text { N.A. } \\
\text { RR: } 31 \\
\text { PP: } 6 \\
\text { SP: } 12 \\
\text { N.A. } \\
\text { RR: } 30 \\
\text { PP: } 6 \\
\text { SP: } 11\end{array}$ & $\begin{array}{l}\text { N.A. } \\
\text { RR: } 5 \pm 7 \\
\text { PP: } 8 \pm 7 \\
\text { SP: } 19 \pm 10 \\
\\
\text { N.A. } \\
\text { RR: } 4 \pm 5 \\
\text { PP: } 7 \pm 6 \\
\text { SP: } 16 \pm 8 \\
\text { N.A. } \\
\text { RR: } 3 \pm 5 \\
\text { PP: } 7 \pm 6 \\
\text { SP: } 16 \pm 9\end{array}$ \\
\hline $\begin{array}{l}\text { Migration } \\
\text { analysis } \\
\text { CXCL13 } \\
M S \\
\text { CXCL10 } \\
\text { MS }\end{array}$ & 7 & $\begin{array}{l}40 \pm 11 \\
43 \pm 11\end{array}$ & $\begin{array}{l}71.4(5: 2) \\
100(7: 0)\end{array}$ & $\begin{array}{l}2 \pm 1.3 \\
2 \pm 1.6\end{array}$ & $\begin{array}{l}\text { UT: 6, GA: } 1 \\
\text { UT: } 6, \text { GA: } 1\end{array}$ & $\begin{array}{l}\text { RRMS } \\
\text { RRMS }\end{array}$ & $\begin{array}{l}5 \pm 8 \\
5 \pm 6\end{array}$ \\
\hline $\begin{array}{l}\text { Proliferation } \\
\text { and activation } \\
\text { assay } \\
\text { HC }\end{array}$ & 3 & $26 \pm 3$ & $100(3: 0)$ & N.A. & N.A. & N.A. & N.A. \\
\hline
\end{tabular}




\subsubsection{Cell isolation}

Peripheral blood mononuclear cells (PBMC) were collected by Ficoll density gradient centrifugation (Lympholyte, Cedarlane Laboratories, Uden, The Netherlands) from whole blood. For phenotyping of DN B cells via flow cytometry, PBMC of MS patients and HC were cryopreserved and thawed before use. PBMC were cryopreserved in 10\% dimethyl sulfoxide (DMSO, Sigma Aldrich, Overijse, Belgium) in fetal bovine serum (FBS, Life Technologies, Gent, Belgium) using a slow temperature-lowering method (Coolcell ${ }$, VWR, Haasrode, Belgium). After $24 \mathrm{~h}$, cryovials were transferred to liquid $\mathrm{N}_{2}$ until analysis. PBMC were thawed by bringing the temperature of the cryovials to $0^{\circ} \mathrm{C}$ in a water bath $\left(37^{\circ} \mathrm{C}\right)$. Hereafter, cold thawing medium consisting of 20\% FBS in RPMI 1640 (Lonza, Basel, Switzerland) was added and PBMC were centrifuged at $4^{\circ} \mathrm{C}$. PBMC were suspended in thawing medium in a concentration of $10 \times 10^{6}$ cells $/ 1000 \mu$ containing DNAse $(0.5 \mathrm{mg} / \mathrm{ml})$ and incubated at $37^{\circ} \mathrm{C}$ for $10 \mathrm{~min}$. Subsequently, cells were washed twice.

For the in vitro chemotaxis and proliferation assays, $\mathrm{CD} 27^{+}$and $\mathrm{CD} 27^{-}$cells were directly purified from freshly isolated PBMC by positive and negative magnetic selection, respectively. Hereafter, $B$ cells were isolated by negative magnetic selection (EasySep ${ }^{\mathrm{TM}}$ Human memory $B$ cell isolation kit, STEMCELL Technologies SARL, Grenoble, France) according to the manufacturer's instructions. Purity of $\mathrm{CD}_{2} 7^{+}$and $\mathrm{CD}^{-} 7^{-} \mathrm{B}$ cells was routinely $\geq 94 \%$ and $93 \%$ respectively, as determined by flow cytometry analysis using the following anti-human antibodies: CD19 PE-Cy7 (BioLegend) and CD27 APC (BD Biosciences, Erembodegem, Belgium), following the staining procedure prior to sorting described below.

\subsubsection{Flow cytometry}

PBMC were incubated for $30 \mathrm{~min}$ at $4^{\circ} \mathrm{C}$ with the fixable viability dye eFluor 506 (1:1000 in PBS; eBioscience, San Diego, USA) to gate for viable lymphocytes. To analyze developmental marker expression, PBMC were stained with anti-human CD20 APC-Cy7, CD19 BV421, IgD PE-Cy7, CD95 PE-Dazzle 594, IgM PerCP-Cy5.5, CD5 BV605, CD38 BV711 (Biolegend, Antwerp, Belgium), IgG FITC, CD10 BV786, CD27 APC (BD Biosciences, Erembodegem, Belgium) and IgA PE (Miltenyi Biotec, Leiden, The Netherlands). 
To measure functional marker expression, PBMC were stained with anti-human CD19 BV421, IgD PE-Cy7, IgM BV605, CD183 BV711 (CXCR3), CD185 APC-Cy7 (CXCR5), CD11a PE (LFA-1), CD49d PerCP-Cy5.5 (VLA-4) (Biolegend), CD166 BV786 (ALCAM), CD27 APC and IgG AF700 (BD Biosciences). T-bet expression was measured by first performing a surface staining using the following antihuman antibodies: CD19 PE-Cy7 (BioLegend), CD27 APC, IgD PE-CF594, IgG FITC, CD21 PE (BD Biosciences), CD11C APC-Cy7 and IgM BV605 (BioLegend). PBMC were then fixed and permeabilized using the Foxp3/Transcription Factor Staining Buffer Set (eBioscience) and stained with anti-human T-bet PerCP-Cy5.5 antibody (eBioscience, Oregon, USA).

The cut-off to identify donors with increased frequencies of DN B cells was $7 \%$ as determined by the mean percentage DN B cells from $\mathrm{HC}<60$ years used for the phenotypic analysis of developmental markers $(n=48)$ plus two times the standard deviation (68). Fluorescence minus one (FMO) controls were used for gating. All flow cytometric analyses were performed on a LSRFortessa flow cytometer and analyzed with FACSDiva software (BD Biosciences) or FlowJo (FlowJo LLC, Ashland, Oregon).

\subsubsection{Chemotaxis assay}

A transwell system with a pore size of $5 \mu \mathrm{M}$ (Corning, NY, USA) was used. In the lower chamber, CXCL13 or CXCL10 (1000 ng/ml, PeproTech, Londen, UK) was added to the chemotaxis buffer (RPMI-1640 medium (Lonza, Basel, Switzerland), $0.5 \%$ bovine serum albumin (BSA; Biowest, Nuaillé, France)) in a total volume of $500 \mu \mathrm{l}$. A total of $1 \times 10^{5} \mathrm{CD} 27^{-}$or $\mathrm{CD} 27^{+} \mathrm{B}$ cells were seeded in duplicate into the upper chamber in a total volume of $100 \mu$ l chemotaxis buffer. After $24 \mathrm{~h}$ of incubation at $37^{\circ} \mathrm{C}$ and $5 \% \mathrm{CO}_{2}$, the cells that migrated to the lower chamber were collected and counted with an automated cell counter (Moxi Z; Witec AG, Sursee, Switzerland). Using flow cytometry the percentages of IgD+CD27- naive, IgD ${ }^{-C D} 27^{-} \mathrm{DN}, \mathrm{IgD}^{-} \mathrm{CD} 27^{+} \mathrm{CSM}$ and $\mathrm{IgD}^{+} \mathrm{CD} 27^{+}$non CSM (NCSM) B cells were determined. The chemotactic index (CI) for each subtype was defined as:

$C I=\frac{\text { number of migrated cells with chemokine-number of migrated cells without chemokine }}{\text { original number of cells in upper chamber }}$ 
During the optimization of this assay, CD27- and $C D 27^{+} \mathrm{B}$ cells were stimulated with $2 \mu \mathrm{g} / \mathrm{ml} \mathrm{CpG2006}$ (ODN2006, Invivogen, Toulouse, France) in combination with $10 \mathrm{ng} / \mathrm{ml}$ interleukin (IL)-4 (R\&D systems, Abingdon, United Kingdom) or 1 $\mu \mathrm{g} / \mathrm{ml} \mathrm{CD40} \mathrm{ligand} \mathrm{(Enzo} \mathrm{Life} \mathrm{Sciences,} \mathrm{Brussels,} \mathrm{Belgium)} \mathrm{together} \mathrm{with} 10 \mathrm{ng} / \mathrm{ml}$ IL-4 for 4,8 or $24 \mathrm{~h}$. Furthermore, different concentrations of CXCL13 and CXCL10 (100 ng/ml, $500 \mathrm{ng} / \mathrm{ml}$ and $1000 \mathrm{ng} / \mathrm{ml}$ ) were tested using total B cells of $\mathrm{HC}$ in combination with $24 \mathrm{~h}$ of migration. The absence of $\mathrm{B}$ cell activation due to incubation in the presence of chemokine was verified by labeling, CD27- and $\mathrm{CD}^{2} 7^{+} \mathrm{B}$ cells with $1 \mu \mathrm{M}$ 5,6-carboxy fluorescein diacetate succinimidyl ester (CFSE, Life technology, California, USA) and incubating them with $1000 \mathrm{ng} / \mathrm{ml}$ CXCL10 or CXCL13 in a 96-well plate for $24 \mathrm{~h}$. Proliferation was then assessed by flow cytometry.

\subsubsection{Proliferation and activation assay}

Tag-it Violet-labeled (Biolegend) $\mathrm{CD} 27^{-}$and $\mathrm{CD} 27^{+} \mathrm{B}$ cells were seeded in a roundbottom 96-well plate (Greiner BioOne, Vilvoorde, Belgium) at $1 \times 10^{5}$ cells with autologous irradiated (8275 rad) PBMC (1:1 ratio) in triplicate. Culture medium was RPMI-1640 medium (Lonza) supplemented with $10 \%$ fetal bovine serum (FBS, Life Technologies), $50 \mathrm{U} / \mathrm{ml}$ penicillin, $50 \mathrm{mg} / \mathrm{ml}$ streptomycin (Invitrogen, Carlsbad, CA), $0.1 \mathrm{mM}$ nonessential amino acids and $1 \mathrm{mM}$ sodium pyruvate (Sigma-Aldrich). Cells were stimulated with $1 \mu \mathrm{g} / \mathrm{ml} \mathrm{CpG2006} \mathrm{(Invivogen)} \mathrm{and} 50$ $\mathrm{U} / \mathrm{ml}$ IL-2 (Sigma-Aldrich), whereas culture medium was included to define background proliferation. After 10 days, cells were collected to assess activation by using flow cytometry using anti-human CD19 PE-Cy7 (BioLegend), CD27 APC, IgD PE-CF594, CD80 PE (BD Biosciences) and CD25 APC-Cy7 (Biolegend), cell proliferation (Tag-it Violet dilution) and fixable viability dye (FVD, eFluor506) of naive, DN, NCSM and CSM $B$ cells. The proliferation fraction $(\triangle P F, \%)$ was determined by subtracting the mean percentage of background proliferation from the mean percentage of proliferation in response to CpG2006. A significant proliferative response was noticed when $\Delta P F \geq 2 \%$, similar to thresholds set for positive proliferation previously documented by our research group (341). Analysis was done on a LSRFortessa ${ }^{\mathrm{TM}}$ flow cytometer using FACSDiva ${ }^{\mathrm{TM}}$ software (BD Biosciences). 


\subsubsection{Statistics}

Statistical analyses were performed using GraphPad Prism 8. A Kruskal-Wallis one-way ANOVA or a Friedman test was used to compare multiple groups. A MannWhitney $U$ test was used for statistical analysis to compare two groups with independent samples. A p value of less than 0.05 was considered statistically significant. 


\subsection{Results}

\subsubsection{Total B cells and DN B cells are increased in the peripheral blood of MS patients}

We evaluated the frequency of total B cells, naive B cells, CSM B cells and DN B cells in the peripheral blood of $\mathrm{HC}(n=48)$, RRMS patients $(n=63)$, SPMS patients ( $n=20)$ and PPMS patients $(n=13)$. The frequency of total B cells was significantly increased in MS patients (RRMS $p=0.004$, PPMS $p=0.041$ and SPMS $\mathrm{p}=0.002$, Figure 4.1(a)) compared to $\mathrm{HC}$, while no difference was detected in the frequency of naive (Figure $4.1(\mathrm{~b})$ ) or CSM B cells (Figure 4.1(c)). The frequency of DN B cells was significantly elevated in RRMS patients ( $n=63, p=$ $0.0035)$ compared to $\mathrm{HC}(\mathrm{n}=42)$, all younger than 60 years (Figure $4.1(\mathrm{~d}-\mathrm{e})$ ). Furthermore, the proportion of RRMS patients $(18 / 63 ; 28.6 \%)$ and PPMS patients $(4 / 11 ; 36.4 \%)$ younger than 60 years with increased peripheral blood frequencies of DN B cells ( $>7 \%$ of CD19+ B cells) was significantly elevated when compared to age-matched $\mathrm{HC}(4 / 42 ; 9.5 \%, \mathrm{p}=0.027$ and $\mathrm{p}=0.048$, respectively, Figure 4.1(f)). Together, these data show an increased frequency of DN B cells in $29.5 \%$ $(26 / 88)$ of the MS patients younger than 60 years compared to $9.5 \%$ of the HC younger than 60 years.
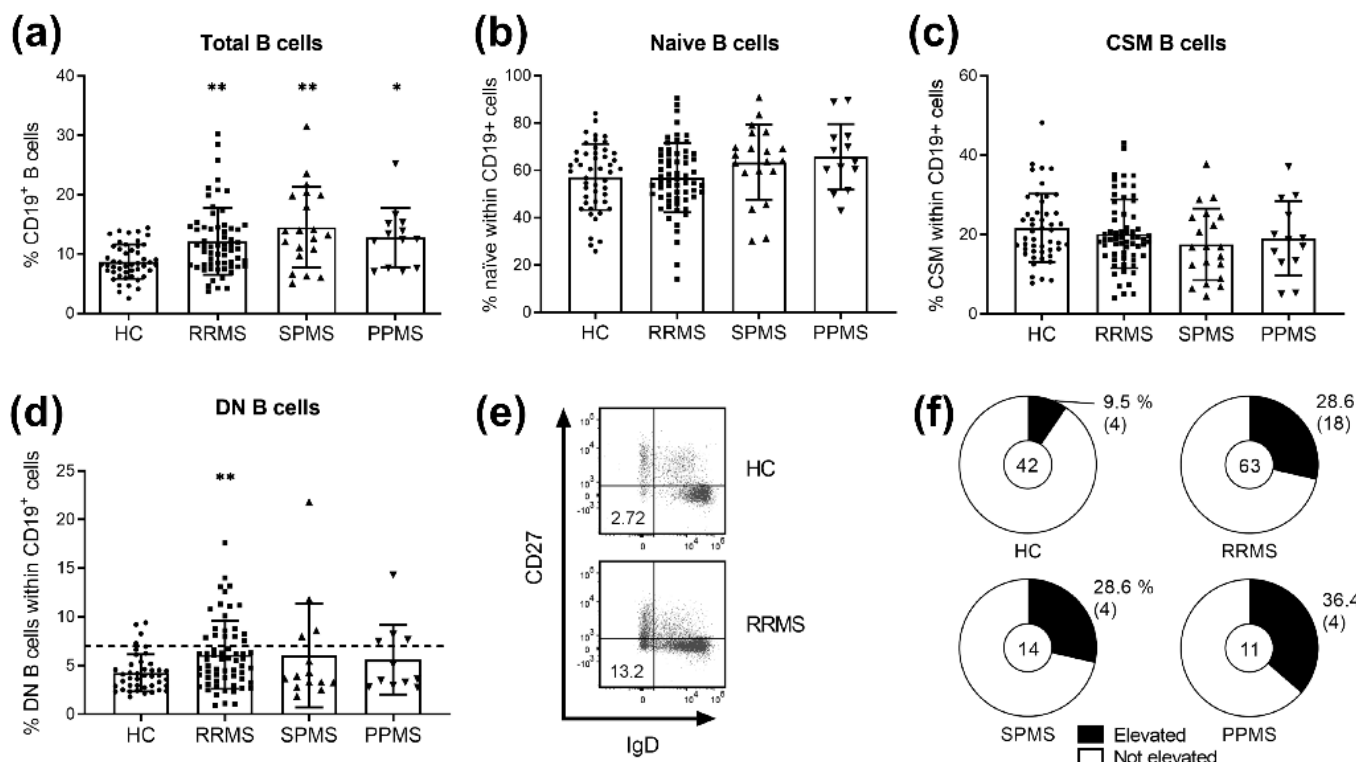

(f)

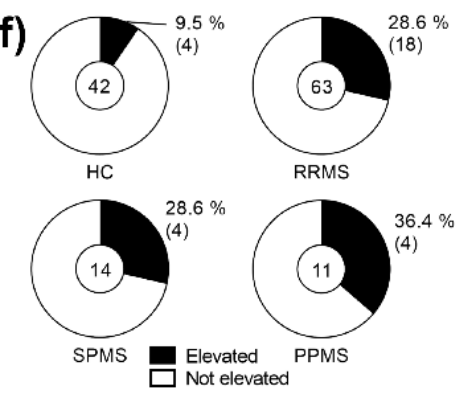




\section{Figure 4.1 Total B cells and DN B cells are increased in MS patients} compared to HC. Frequency of $(\mathbf{a})$ total B cells $\left(\mathrm{CD} 19^{+}\right)$, (b) naive B cells $\left(\operatorname{IgD}{ }^{+} \mathrm{CD} 27^{-}\right)$ and (c) CSM B cells $\left(\operatorname{IgDCD}^{-} 7^{+}\right)$in the peripheral blood of HC $(n=48)$, RRMS $(n=63)$, SPMS $(n=20)$ and PPMS ( $n=13)$ patients. (d) Frequency of DN B cells (IgD-CD27 $)$ in the peripheral blood of HC $(n=42)$, RRMS $(n=63)$, SPMS $(n=14)$ and PPMS $(n=11)$ patients younger than 60 years. Mean (bars) $\pm S D$ is depicted. Black dashed line represents the cutoff. (e) Representative plots of a $\mathrm{HC}$ without and a RRMS patient with an increased frequency of DN B cells. (f) Percentage of HC, RRMS, SPMS and PPMS patients younger than 60 years with and without an elevated DN B cell frequency ( $>7 \%$ of $\mathrm{CD} 19^{+} \mathrm{B}$ cells). The number in the central oval indicates the number of individuals examined. The percentage and the number (in parentheses) of individuals with an increased DN B cell frequency are depicted. A Kruskal-Wallis one-way ANOVA was used to compare the different groups. A Mann-Whitney $U$ test was used for statistical analysis to compare 2 groups. Fisher's exact test was used to compare proportions between study groups. * $\mathrm{p}<0.05, * * \mathrm{p}<0.01 \mathrm{HC}=$ healthy control, MS = multiple sclerosis, RRMS = relapsing-remitting MS, SPMS = secondary progressive MS, PPMS = primary progressive MS, CSM = class-switched memory, DN = double negative.

\subsubsection{DN B cells resemble CSM $B$ cells in the expression of developmental surface markers}

Further phenotypic analysis was done by measuring the distribution of Ig isotypes (IgM, IgG, IgA) and developmental markers (CD5, CD38, CD10, CD95) on DN, CSM and naive B cells of HC $(n=48)$ and MS patients $(n=96$; RRMS $n=63$, PPMS $n=13$, SPMS $n=20$, Figure 4.2(a)). The largest proportion of DN B cells was $\mathrm{IgG}^{+}$in both $\mathrm{HC}$ and $\mathrm{MS}$ patients (46 \pm 14 and $45 \pm 14 \%$, respectively), followed by an equal proportion of $\mathrm{IgM}^{+}$cells $(20 \pm 16$ and $22 \pm 16 \%$, respectively) and $\mathrm{IgA}^{+}$cells (20 \pm 10 and $20 \pm 9 \%$, respectively) (Fig. 4.2(b)). DN B cells were similar to CSM B cells in the frequency of $\mathrm{IgM}^{+}$and $\mathrm{IgG}^{+}$cells in $\mathrm{HC}$ and MS patients (Figure 4.2(b)). However, the frequency of $\operatorname{IgA}^{+}$DN B cells was lower compared to IgA ${ }^{+}$CSM B cells in HC and MS patients $(p<0.0001$, Figure 4.2(b)). DN B cells showed the same expression pattern of Ig isotypes when comparing $\mathrm{HC}$ with MS and within the different MS populations (RRMS, PPMS, SPMS; data not shown). Further, the frequency of $\mathrm{CD}^{+}, \mathrm{CD} 38^{+}$and $\mathrm{CD} 95^{+}$ cells in the DN B cell population was in between that of CSM and naive B cells (Figure 4.2(c-d)). 
In this regard, naive $\mathrm{B}$ cells presented the highest frequency of $\mathrm{CD}^{+}$and $\mathrm{CD} 38^{+}$ cells whereas CSM B cells demonstrated the highest frequency of CD95 ${ }^{+}$cells. No difference was detected in the frequency of $\mathrm{CD} 10^{+} \mathrm{DN}$ B cells compared to CD10+ CSM or naive B cells (Figure 4.2(c-d)). These results were consistent in the different MS subpopulations (RRMS, SPMS, PPMS) (data not shown). We can conclude that DN B cells show a mature phenotype and seem to be less activated since their expression of activation marker CD95 is lower compared to CSM B cells. Furthermore, the expression of developmental markers, activation markers and Ig isotypes on DN B cells was similar between HC and MS patients and between the different MS subpopulations (RRMS, PPMS, SPMS). 
A

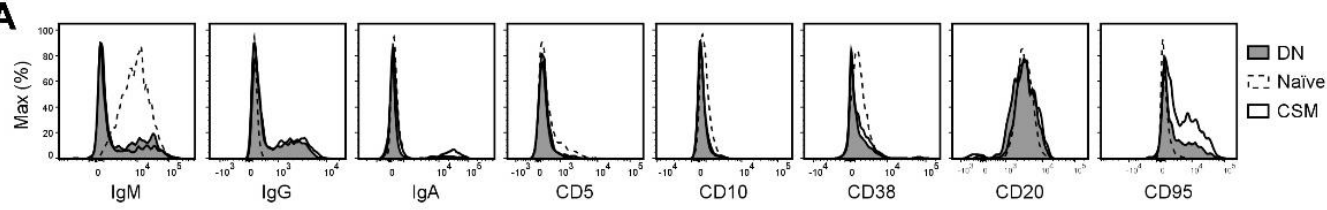

B

$\lg M$

IgG

$\lg \mathrm{A}$

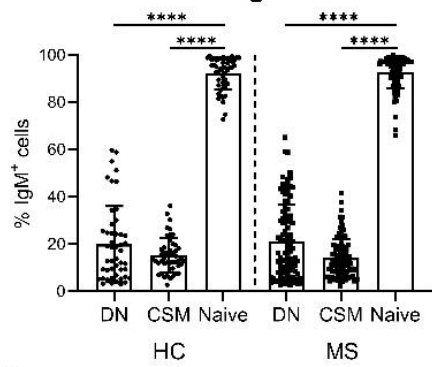

C

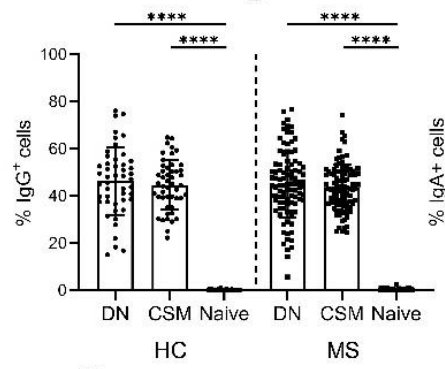

D
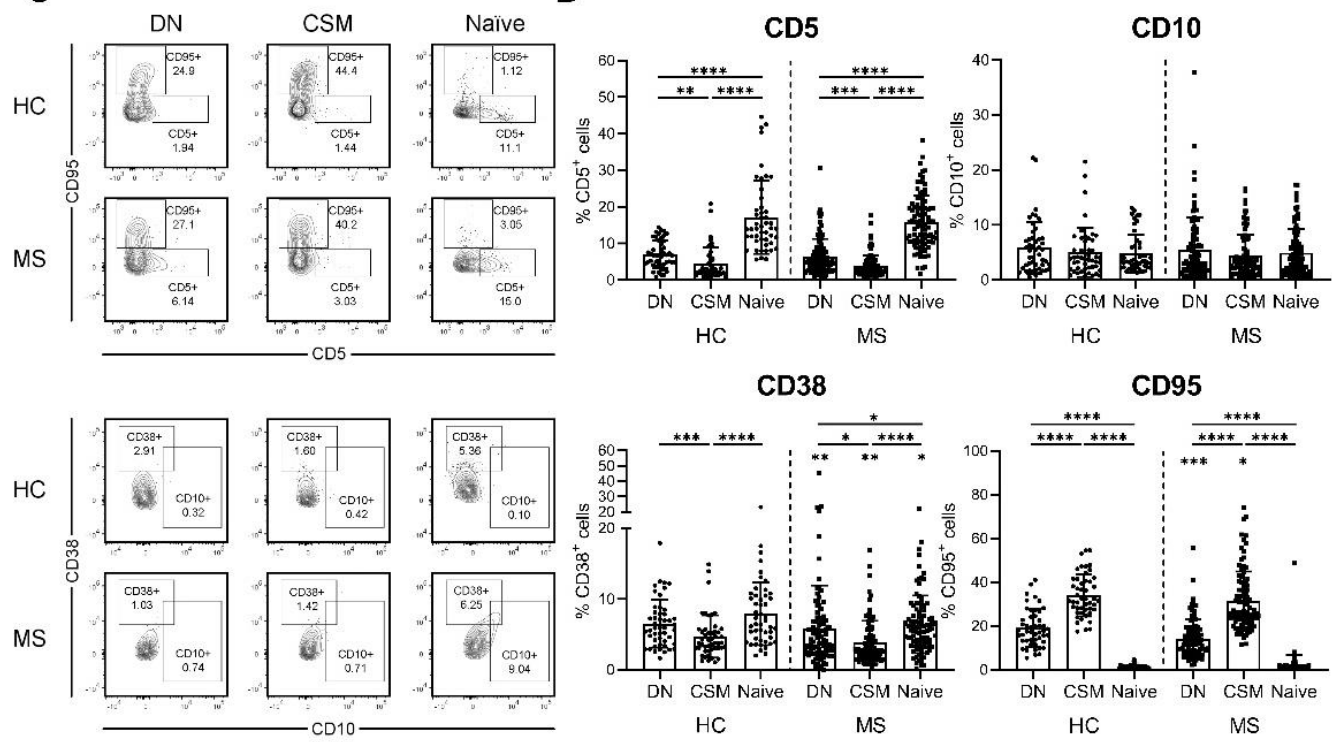

Figure 4.2 DN B cells of HC and MS patients resemble CSM B cells in the expression of developmental surface markers, however in an earlier maturation state. (a) Representative flow cytometry plots of the expression level of the indicated markers on DN, naive and CSM B cells. (b) Frequency of $\mathrm{IgM}^{+}$, IgG ${ }^{+}$and IgA ${ }^{+}$cells within the DN, CSM and naive B cell subtypes in the peripheral blood of $\mathrm{HC}(\mathrm{n}=48)$ and MS patients $(n=96)$. Mean $\pm S D$ is depicted. (c) Representative flow cytometry plots of the expression patterns of CD95 versus CD5 and CD38 versus CD10 for DN, CSM and naive $B$ cells of a $\mathrm{HC}$ and MS patient. 
(d) Frequency of $\mathrm{CD}^{+}, \mathrm{CD} 10^{+}, \mathrm{CD} 8^{+}$and $\mathrm{CD} 95^{+}$cells within the $\mathrm{DN}, \mathrm{CSM}$ and naive $\mathrm{B}$ cell substypes in the peripheral blood of HC $(n=48)$ and MS patients $(n=96)$. Mean \pm SD is depicted. A Kruskal-Wallis one-way ANOVA or a Friedman test was used to compare multiple groups. A Mann-Whitney $\mathrm{U}$ test was used for statistical analysis to compare 2 groups. * $\mathrm{p}<$ $0.05, * * \mathrm{p}<0.01, * * * \mathrm{p}<0.001, * * * * \mathrm{p}<0.0001$. HC $=$ healthy control, MS = multiple sclerosis, CSM = class- switched memory, $\mathrm{DN}=$ double negative.

\subsubsection{CD21 low $B$ cells show the highest expression of T-bet, while a proportion of DN B cells express T-bet}

We measured T-bet expression in DN, naive, CD21 1ow, NCSM and CSM B cells of $\mathrm{HC}$ and untreated MS patients via flow cytometry (Figure 4.3). The frequency of T-bet $^{+}$cells was significantly higher in the DN B cell population compared to the naive $(p<0,0001)$ and the NCSM B cell populations ( HC $p=0,0164$; MS $p=$ 0,0088, Figure 4.3(a-b)). The expression level (MFI) of T-bet was also significantly higher in the DN B cell population compared to the naive ( $\mathrm{HC} p=0,0013$; MS $p$ $<0,0001$ ) and NCSM B cell populations (HC $p=0,0013$; MS $p<0,0001$, Figure 4.3(a-b)). Further, the expression level (MFI) of T-bet was higher on DN B cells compared to CSM B cells of MS patients ( $p=0.0005$, Figure 4.3(b)). The CD2 $1^{\text {low }}$ $B$ cell populations showed the highest frequency $(\mathrm{HC}=75,72 \%$ and $\mathrm{MS}=$ $69,27 \%$ ) and T-bet expression level (MFI HC $=9287$ and MS $=9095$ ) of T-bet ${ }^{+}$ cells, which was significantly higher compared to DN B cells in HC (frequency $p=$ 0,0191 and MFI $p=0,0349$ ) and MS patients (frequency $p<0,0001$ and MFI $p=$ 0,0012 , Figure 4.3(a-b)). Furthermore, the expression pattern of T-bet on naive, NCSM, CSM, DN and CD21 low B cells was similar between the different MS populations (RRMS, SPMS, PPMS; data not shown). In conclusion, the majority (approximately $71 \%$ ) of T-bet $^{+} \mathrm{B}$ cells belong to the CD21 low phenotype, with about $23 \%$ of DN B cells being T-bet ${ }^{+}$in $\mathrm{HC}$ and MS patients. 
(a)

\section{Percentage of positive cells}

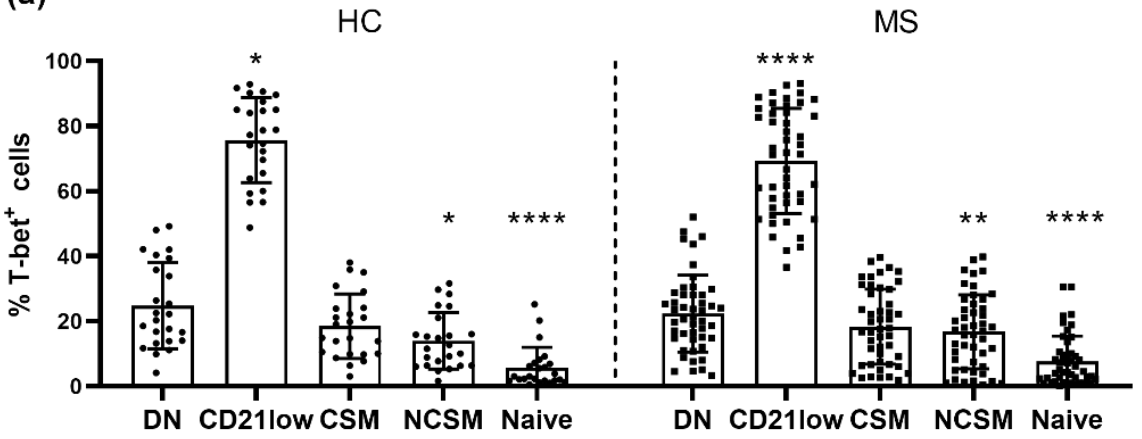

MFI

(b)

$\mathrm{HC}$

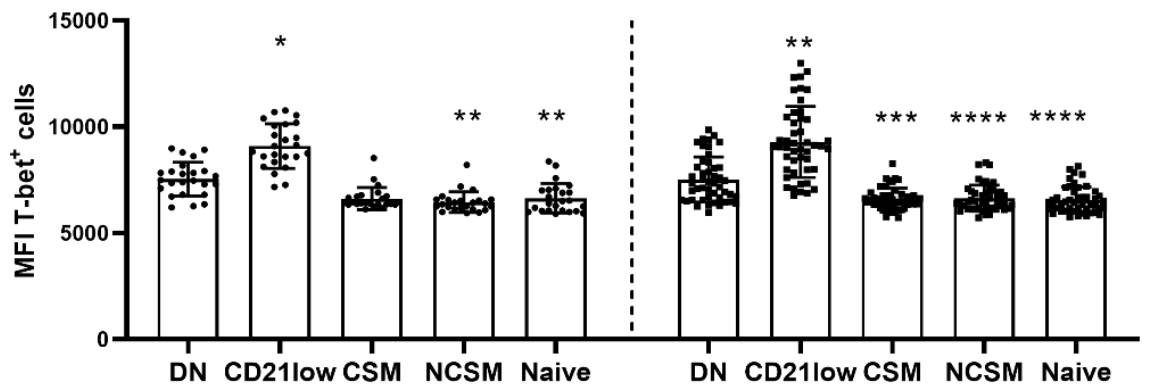

Figure 4.3 CD21 ${ }^{\text {low }} \mathrm{B}$ cells show the highest frequency and expression of T-bet in HC and MS patients. Frequency (a) and mean fluorescence intensity (MFI) (b) of T-bet ${ }^{+}$cells within naive, NCSM, CSM, DN and CD21 $1^{\text {low }} B$ cell populations of HC $(n=$ 24) and MS patients $(n=47)$. Mean \pm SD is depicted. A Friedman test was used to compare multiple groups. A Mann-Whitney $\mathrm{U}$ test was used for statistical analysis to compare 2 groups. $* \mathrm{p}<0.05, * * \mathrm{p}<0.01, * * * \mathrm{p}<0.001, * * * * \mathrm{p}<0.0001$. MFI: mean fluorescence intensity, $\mathrm{HC}=$ healthy control, MS $=$ multiple sclerosis, NCSM $=$ non class- switched memory, CSM = class-switched memory, DN = double negative.

\subsubsection{The expression of adhesion molecules and chemokine receptor} CXCR3 of DN B cells is between that of naive and CSM B cells.

We analyzed the expression of adhesion molecules and chemokine receptors on peripheral blood DN, CSM and naive B cells of HC and untreated MS patients (Figure 4.4(a)). We indicated that the expression level (MFI) of adhesion molecules LFA-1, VLA-4 and ALCAM on DN B cells was in between that of CSM and 
naive $B$ cells (Figure 4.4(b)). In this regard, naive B cells presented with the lowest expression level of adhesion molecules, whereas CSM B cells demonstrated the highest expression level of adhesion molecules (Figure 4.4(b)). Furthermore, we observed that the frequency of $\mathrm{CXCR}^{+}$DN B cells was in between that of CSM (MS: $p<0.0001$ ) and naive B cells (MS: $p<0.0001$, Figure 4.4(c)). In contrast, we indicated that the frequency and expression levels (MFI) of $\mathrm{CXCR}^{+}$cells within the DN $B$ cell population were lower than those within the naive $(p<0.0001)$ and CSM B cell populations (Frequency $p \leq 0.0001$, MFI HC $p=0.0486$ and MFI MS $p$ $=0.1648$ ) of $\mathrm{HC}$ and MS patients (Figure 4.4(c)). Furthermore, the expression patterns of the adhesion markers and chemokine receptors of DN, CSM and naive $B$ cells were similar between $\mathrm{HC}$ and MS patients and the different MS populations (RRMS, PPM, SPMS; data not shown). In conclusion, DN B cells expressed adhesion molecules (LFA-1, VLA-4 and ALCAM) and chemokine receptors CXCR3 and CXCR5. Furthermore, DN $B$ cells showed an intermediate expression of adhesion molecules LFA-1, VLA-4 and ALCAM and chemokine receptor CXCR3 between naive and CSM $B$ cells. 
(a)
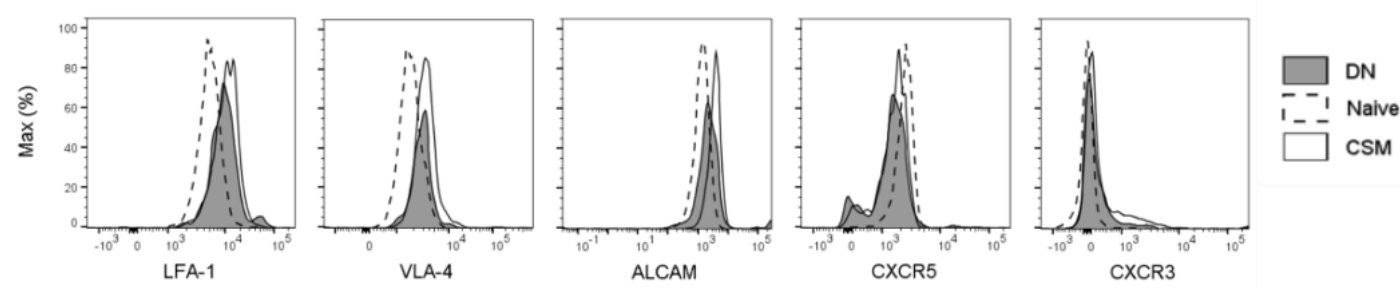

(b)

LFA-1

VLA-4

ALCAM
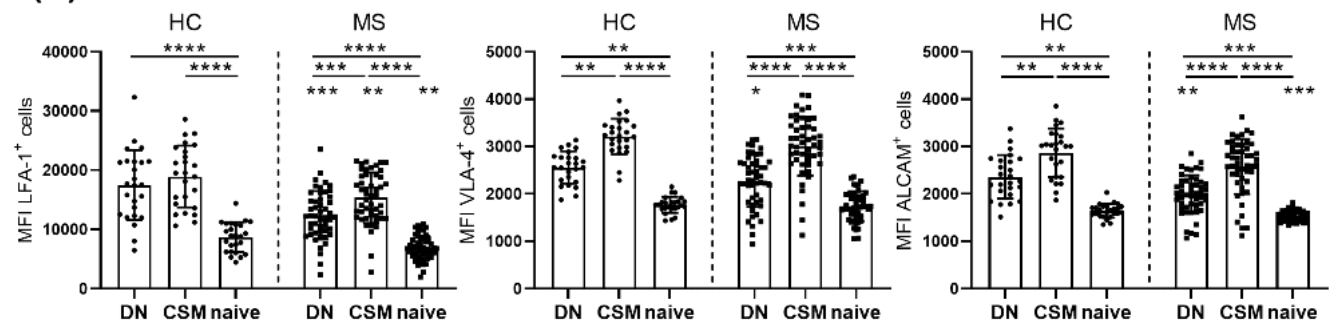

(c)

CXCR3

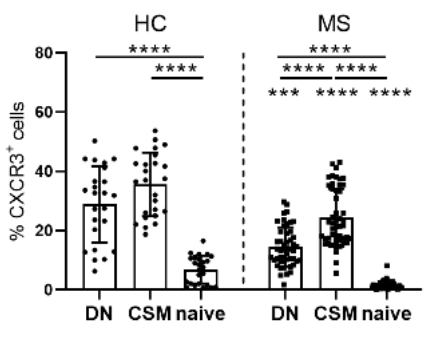

CXCR3

CXCR5
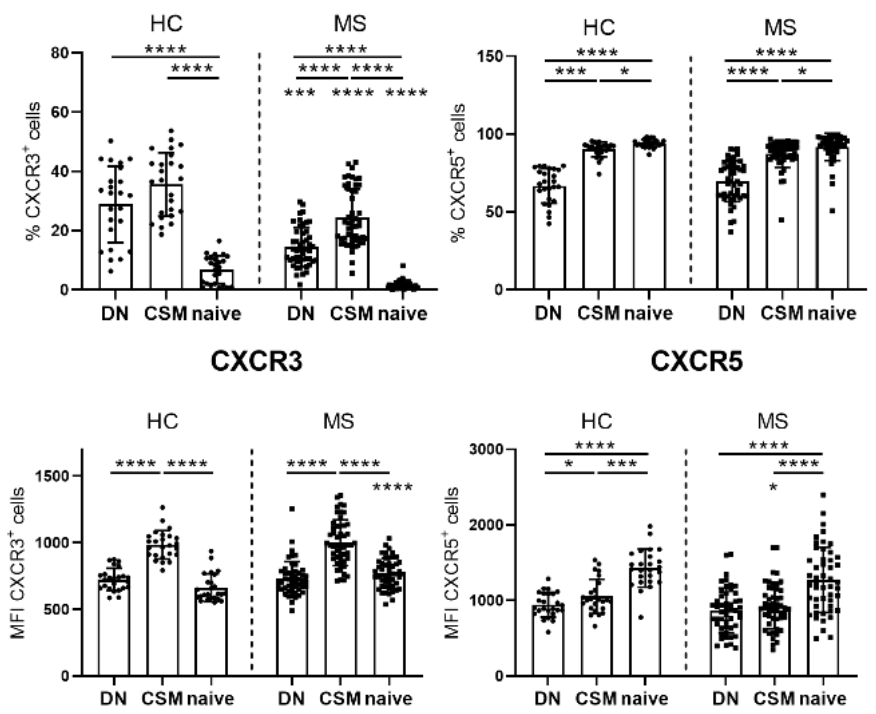

Figure 4.4 DN B cells of HC and MS patients show an intermediate expression of adhesion molecules and chemokine receptor CXCR3 between naive and CSM B cells. (a) Representative flow cytometry plots of the expression level of the indicated markers on DN, naive and CSM B cells. (b) Expression level (MFI) of the adhesion molecules LFA-1, VLA-4 and ALCAM on DN, CSM and naive B cells from the peripheral blood of $\mathrm{HC}(n=25)$ and MS patients $(n=49)$. (c) Frequency of CXCR3 ${ }^{+}$ and $\mathrm{CXCR5} 5^{+}$cells and expression level (MFI) of CXCR3 and CXCR5 within DN, CSM and naive 
B cell subtypes from the peripheral blood of HC $(n=25)$ and MS patients $(n=49)$. Mean \pm $\mathrm{SD}$ is depicted. A Friedman test was used to compare multiple groups. A Mann-Whitney $U$ test was used for statistical analysis to compare 2 groups. $* \mathrm{p}<0.05, * * \mathrm{p}<0.01, * * * \mathrm{p}$ $<0.001, * * * * \mathrm{p}<0.0001$. MFI: mean fluorescence intensity, $\mathrm{HC}=$ healthy control, MS = multiple sclerosis, CSM = class-switched memory, DN = double negative, LFA-1 = lymphocyte function-associated antigen-1, VLA-4 = very late antigen-4, ALCAM = activated leukocyte cell adhesion molecule, CXCR3 = chemokine receptors CXC receptor 3, CXCR5 = chemokine receptors CXC receptor 5 .

\subsubsection{DN B cells migrate towards CXCL13 and CXCL10 in vitro}

As a proportion of DN B cells showed expression of the chemokine receptors CXCR3 and CXCR5, we now tested whether DN B cells might have the capacity to migrate towards the corresponding pro-inflammatory chemokines CXCL10 and CXCL13. Therefore, an in vitro chemotaxis assay was performed to examine the migration capacity of naive, DN, NCSM, and CSM B cells from untreated RRMS patients towards CXCL13 $(n=7)$ and $\operatorname{CXCL10}(n=7)$.

In order to perform the chemotaxis assay, the assay was first optimized. The following parameters were tested: polyclonal stimulation (CD40L + IL-4, CpG2006 $+\mathrm{IL}-4)$, duration of the migration assay (4h, $8 \mathrm{~h}$ and $24 \mathrm{~h}$ ) and concentration of chemokines $(100 \mathrm{ng} / \mathrm{ml}, 500 \mathrm{ng} / \mathrm{ml}, 1000 \mathrm{ng} / \mathrm{ml}$ ). We detected that polyclonal stimulation did not increase the migration capacity and chemokine receptor expression of $C D 27^{-}$and $C D 27^{+}$B cells. Furthermore, an incubation time of $24 \mathrm{~h}$ resulted in the highest chemotactic index and number of migrated $B$ cells which is needed for proper flow cytometric analysis. In addition, $1000 \mathrm{ng} / \mathrm{ml} \mathrm{CXCL13}$ or CXCL10 was the optimal concentration to trigger B cell migration in our in vitro assay. To exclude potential proliferation of the four different B cell subtypes (naive, NCSM, CSM and DN) after $24 \mathrm{~h}$ of incubation with the pro-inflammatory chemokines CXCL13 and CXCL10, an in vitro proliferation assay (CFSE dilution) was performed. No proliferation of DN, naive, NCMS and CSM B cells was observed, since the percentage of cells in the proliferated fraction is around 0 $0.5 \%$ (data not shown).

\section{Migration capacity of DN B cells from MS patients}

The migration capacity of naive, DN, NCSM and CSM B cells from untreated RRMS patients towards $\operatorname{CXCL13}(n=7)$ and $\operatorname{CXCL10}(n=7)$ was investigated using the 110 
optimized in vitro chemotaxis assay. The migration capacity of DN B cells from untreated RRMS patients towards CXCL13 (CI: $17.3 \pm 7.3$ ) and CXCL10 (CI: 1.4 \pm 3.8 ; Figure 4.5(a)) was similar to that of CSM B cells $(15.1 \pm 5.1$ and $3.2 \pm$ 2.4, respectively). In addition, migration of DN B cells towards CXCL13 was significantly higher compared to naive (CI: $4.6 \pm 3.9 ; \mathrm{p}<0.01)$ and NCSM B cells (CI: $5 \pm 2.3 ; \mathrm{p}<0.05)$. We also measured the frequency of $\mathrm{CXCR}^{+}$and $\mathrm{CXCR} 5^{+}$ cells within the DN, naive, NCSM and CSM B cell populations in these cohorts of MS patients. Results were similar to what we described above (Figure 4.5(b)). The DN B cell population showed an intermediate frequency of $\mathrm{CXCR}^{+}$cells in between that of naive and CSM B cells and the frequency of CXCR5 ${ }^{+}$cells was lower in the DN B cell population than in naive or CSM B cells. These results indicate that DN B cells of untreated RRMS patients can migrate towards CXCL13 and CXCL10 in vitro with a migration capacity that is similar to that of CSM B cells and express the chemokine receptors CXCR3 and CXCR5.

(a)
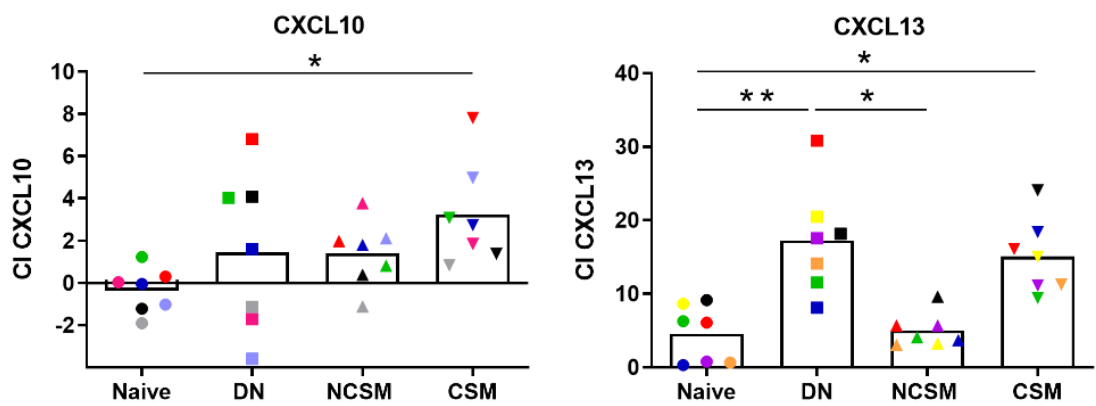

(b)
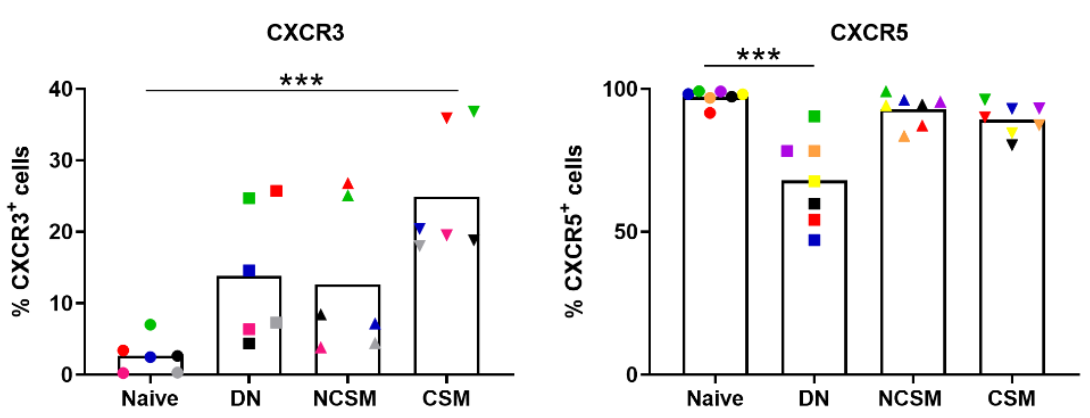

Figure 4.5 DN B cells of MS patients show an in vitro migration capacity towards CXCL10 and CXCL13 that is similar to that of CSM B cells. (a) CI of naive, DN, NCSM and CSM $B$ cells that migrate towards CXCL10 ( $n=7$ RRMS) or CXCL13 
( $n=7$ RRMS). (b) Frequency of $\mathrm{CXCR}^{+}\left(\mathrm{n}=7\right.$ RRMS) and $\mathrm{CXCR}^{+}(\mathrm{n}=7$ RRMS) naive, DN, NCSM and CSM B cells. A Friedman test was used to compare multiple groups. $* \mathrm{p}<$ $0.05, * * \mathrm{p}<0.01, * * * \mathrm{p}<0.001$. CI: chemotactic index; DN: double negative; CSM: classswitched memory; NCSM: non class-switched memory.

\subsubsection{Proliferation and activation profile of DN B cells compared to naive, NCSM and CSM B cells}

DN B cells of MS patients showed a pro-inflammatory cytokine profile (68), were present in the CSF of MS patients (68) and were able to migrate in vitro towards pro-inflammatory chemokines (this thesis). Furthermore, contradictory results exist on the proliferation and activation potential of DN B cells. For this reason, we investigated the proliferation and activation status of DN B cells after TLR9 stimulation $(70,88,342)$.

\section{DN B cells of HC are activated following TLR9 stimulation}

To determine if DN B cells can get activated, Tag-it Violet labeled CD27- or CD27+ B cells of HC ( $n=3)$ were stimulated with CpG2006 or left unstimulated for 10 days. Although statistical analysis was not possible due to an insufficient number of replicates, DN B cells appeared to show a higher proliferative response after CpG2006 stimulation compared to naive and NCSM B cells (Figure 4.6(a)). Furthermore, an increase in the frequency of activated cells was observed following CpG2006 stimulation in the DN B cell population (9-fold for CD80+ cells, 6-fold for $\mathrm{CD}_{2} 5^{+}$cells). In comparison, a lower increase in the frequency of activated cells was observed following CpG2006 stimulation in the NCSM and in the CSM (2-fold for $\mathrm{CD}^{+} 0^{+}$and $\mathrm{CD}^{2} 5^{+}$cells) B cell populations (Figure 4.6(b)). Stimulating DN B cells with CpG2006 resulted in a higher increase in CD80 and CD25 expression levels (MFI; 7.6 and 3.1 times, respectively) in comparison with naive ( 1.5 and 1.1 times, $p=0,0278$, respectively), NCSM (4.0 and 1.8 times, respectively) and CSM (3.0 and 1.9 times, respectively) B cells (Figure 4.6(b)). Together, the results above demonstrate that DN B cells of HC have the ability to proliferate and become activated in response to TLR9 stimulation. 
(a)

(b)
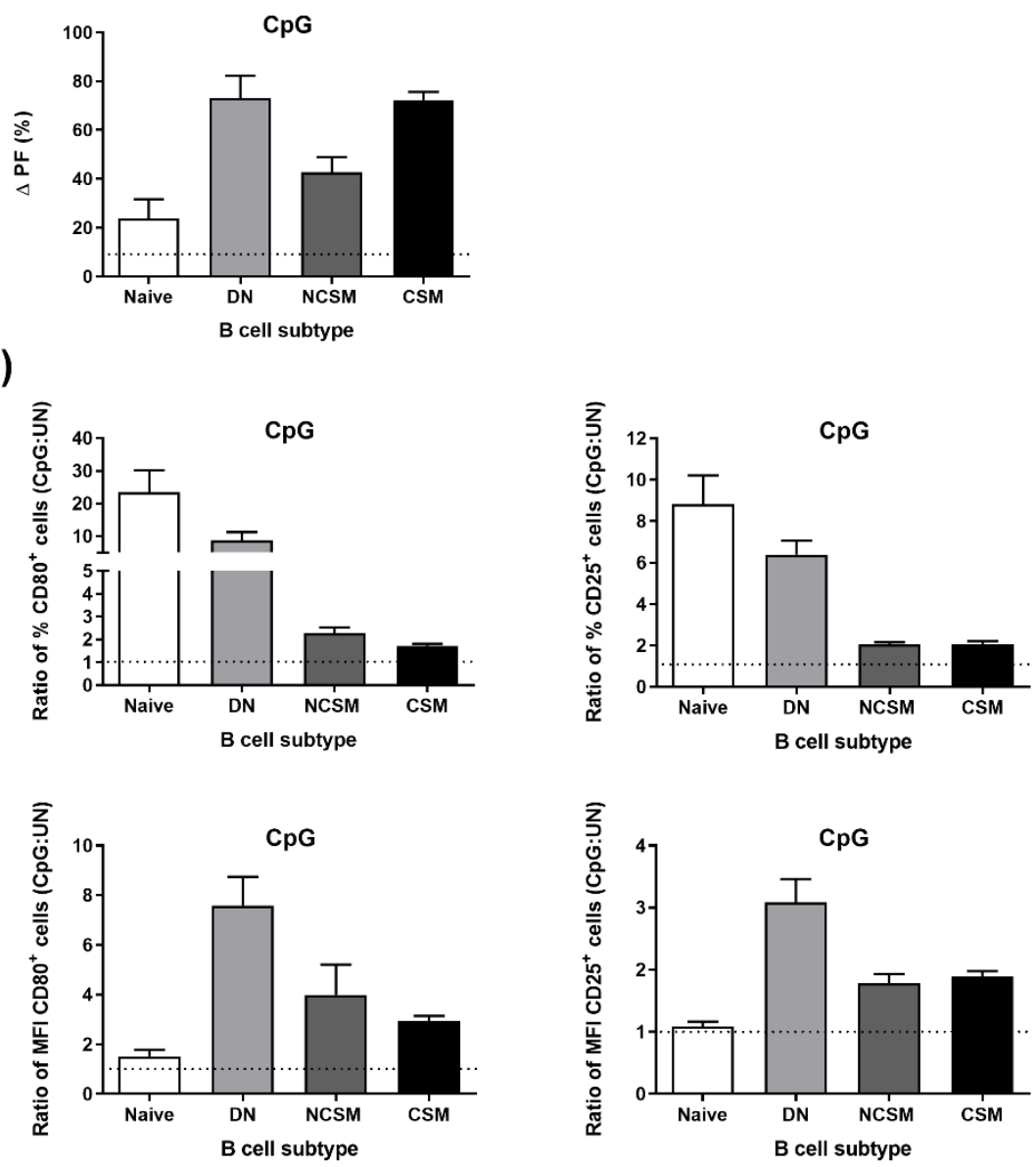

Figure 4.6 Activation potential of naive, DN, NCSM and CSM B cells after TLR9 stimulation. Tag-it violet labeled $\mathrm{CD} 27^{-}$and $\mathrm{CD} 27^{+} \mathrm{B}$ cells of $\mathrm{HC}$ ( $\mathrm{n}$ = 3) were stimulated with CpG2006 or left untreated and cultured for 10 days in culture medium together with irradiated PBMC. (a) The percentage of proliferating naive, DN, NCSM and CSM B cells corrected for background proliferation ( $\triangle P F)$ is depicted. Expression levels of activation markers were assessed via flow cytometry. The $\triangle \mathrm{PF}$ was corrected for background proliferation (i.e. proliferation in the absence of TLR9 stimulation). The cut-off for positive TLR9 proliferation was set at $\geq 2 \%$ and indicated by the dashed line. The ratios (CpG2006 stimulated/unstimulated) of (b) the percentage or MFI of $\mathrm{CD} 80^{+}$and $\mathrm{CD} 25^{+}$cells within naive, DN, NCSM and CSM B cell populations are shown in which the expression in the unstimulated condition is set at 1 . Mean levels + SEM are depicted. DN: double negative; NCSM: non class-switched memory; CSM: class-switched memory. 


\subsection{Discussion}

In this study, we elucidated the developmental and migratory phenotype and function of DN B cells, a B cell subtype that is increased in a proportion of MS patients and is present in the CSF of MS patients. Our data suggest that DN B cells are mature cells that show an expression pattern of developmental markers intermediate between naive and CSM B cells and that these are antigenexperienced by mostly expressing IgG or IgA. Furthermore, DN B cells express pro-inflammatory adhesion molecules and chemokine receptors and have the potential to migrate to pro-inflammatory chemokines CXCL13 and CXCL10 in vitro. In addition, DN B cells express activation markers and are able to respond to in vitro activation.

Our study showed that DN B cells have a mature phenotype, since they are antigen experienced expressing $\mathrm{IgG}^{+}$and $\mathrm{IgA}^{+}$and they expressed low levels of CD38 and the immature markers CD5 and CD10. The distribution of $\mathrm{IgG}^{+}$and $\mathrm{IgM}^{+}$cells was similar in the DN and CSM B cell population, which might implicate that DN B cells show a phenotype that resembles CSM B cells. However, DN B cells appear to be in an earlier maturation state than CSM B cells since their expression of CD5 and CD38 is higher compared to CSM B cells, while the frequency of $\mathrm{IgA}^{+}$and $\mathrm{CD}^{+} 5^{+}$cells was lower compared to CSM. Nonetheless, there is an indication that a proportion of DN B cells has an activated phenotype since the frequency of $\mathrm{CD}^{+} 5^{+}$cells within the DN B cell population was higher compared to naive B cells. Interestingly, in SLE patients, CD95 ${ }^{+}$DN B cells showed mutated and class-switched $B$ cell receptors. These cells were increased in patients with disease flares and correlated with disease activity and serological abnormalities (343). In contrast, the entire DN B cell population did not correlate with disease activity (343). This indicates that it is important to further investigate the role of $\mathrm{CD} 95^{+} \mathrm{DN}$ B cells in the pathology of MS.

We analyzed whether DN B cells of MS patients expressed T-bet, as this could have a potential contribution to the disease manifestation in MS. We demonstrated about $23 \%$ of T-bet $^{+}$DN B cells in RRMS patients, which is higher than the percentage of T-bet ${ }^{+}$naive and NCSM B cells and is comparable to the frequency of T-bet ${ }^{+}$CSM B cells. However, we indicated that about $71 \%$ of CD $21^{\text {low }} \mathrm{B}$ cells express T-bet in RRMS patients. 
No difference in T-bet expression of DN B cells of HC and RRMS patients was found, which is in agreement with the studies of Frisullo et al. (344). Additionally, no difference between patients with relapse and without relapse could be found (344). In accordance with our data, Frasca et al. reported about $10-20 \%$ of Tbet $^{+}$DN B cells in HC (young and old), which was also higher compared to naive and CSM B cells (345). However, we did not observe a significant difference in Tbet expression between DN and CSM B cells, which could be due to the higher number of subjects in our study. Our data indicate that T-bet ${ }^{+}$DN B cells can be found in MS patients. However, CD21 low $B$ cells have the highest expression of Tbet and are more likely to have a common characteristic with $\mathrm{T}^{- \text {bet }^{+}}$ageassociated $B$ cells found in mice and CD11 $c^{\text {hi }} B$ cells of SLE patients $(172,339)$. Nonetheless, about $20 \%$ of DN B cells could be retraced to the CD21 low CD $11 C^{+} B$ cell gate in our previous study (68), meaning that in this subtype of DN B cells there could be a role for T-bet in the functional process of DN B cells. This also indicates that different subtypes of DN B cells, which can exert different functions in the pathology of MS, could exist, or that not all subtypes of DN B cells are involved in the pathology of MS. This notion is supported by a study of Du et al. who demonstrated that functional $\mathrm{CD}_{11 \mathrm{C}^{+}}$age-associated $\mathrm{B}$ cells in mice can also be generated in the absence of $B$ cell T-bet expression (346). T-bet- ageassociated B cells could still secrete IgG that bound to self-ligand dsDNA, indicating that deletion of T-bet does not impact autoreactivity within the ageassociated B cell compartment (346).

DN B cells of MS patients showed an intermediate expression of adhesion molecules (LFA-1, VLA-4 and ALCAM) in between that of naive and CSM B cells. Since these adhesion molecules are involved in the migration into the CNS, these results indicate that DN $B$ cells have a pro-migratory adhesion molecule pattern that could partly explain their increased frequency in the CSF of MS patients (68). This is functionally relevant, as the firm adhesion of B cells to CNS endothelial cells is critically dependent upon the binding of adhesion molecules to their receptors (140).

It has been recently shown that $\mathrm{ALCAM}^{+} \mathrm{B}$ cells show a memory phenotype and express activation markers and pro-inflammatory cytokines (347, 348). Activation of $B$ cells increases the expression of ALCAM, which mediates the 
migration of $\mathrm{B}$ cells. ALCAM+ $\mathrm{B}$ cells are overrepresented in the periphery and cortical lesions of MS patients $(347,348)$. Since DN B cells showed a higher expression of ALCAM compared to naive B cells, we can speculate that this could mediate the migration of DN $B$ cells and is partly responsible for the increased frequency in the CSF. Furthermore, the higher expression of ALCAM supports the notion that DN B cells have a pro-inflammatory memory phenotype.

Next to adhesion molecules, about $15 \%$ of DN B cells of MS patients expressed the chemokine receptor CXCR3 while about 70\% expressed CXCR5. CXCR3 expression levels on MS DN B cells were lower compared to CSM B cells, while CXCR5 was equally expressed on DN and CSM B cells. Corresponding to our results, DN B cells of young $\mathrm{HC}$ were previously shown to have higher CXCR3 expression levels than naive B cells (349). In contrast, CSM B cells showed lower CXCR3 compared to DN B cells. Furthermore, Bulati et al. reported that DN B cells expressed CXCR5, although it was very low (349). We also observed reduced CXCR5 expression (\% and MFI) on DN B cells compared to naive and CSM B cells. Furthermore, $C D 11 C^{\text {hi }} B$ cells, a subtype involved in the pathology of the autoimmune disease SLE, also showed an increased expression of CXCR3 and a decreased expression of CXCR5 compared to naive B cells (339). One of the reasons why CXCR5 has the highest expression on naive $B$ cells could be because in healthy individuals, CXCR5 drives naive B cells to lymph nodes in search of their cognate antigen $(339,350)$. However, in MS patients, CXCL13 is increased in the CSF and this increase is linked with CNS accumulation of CSM B cells and DN $B$ cells. In the CSF, nearly all B cells express CXCR5 $(129,340)$. A possible mechanism for the reduced CXCR5 expression on DN $B$ cells could be the increased expression of CXCL13 at the inflammatory site where DN B cells can reside, which possibly caused internalization of CXCR5 (351). Another possibility is that CXCR5+ DN B cells reside in the CNS instead of in the peripheral blood. Together, these data suggest a role for the chemokines CXCL10 and CXCL13 in the migration of DN B cells towards the CNS.

Using an in vitro chemotaxis assay, we showed that DN B cells of MS patients have a high migration potential towards the pro-inflammatory chemokines CXCL10 and CXCL13. Their migration towards CXCL13 was comparable to that of CSM B cells and higher than that of naive and NCSM B cells. Thus, DN B cells of 
MS patients could migrate towards pro-inflammatory sites where high levels of CXCL10 and CXCL13 are present. Although DN B cells showed a lower expression of CXCR5 than naive B cells, their migration towards CXCL13 was more pronounced. No correlation could be found between the expression levels of CXCR5 or CXCR3 on B cell subtypes and their migration towards CXCL13 and CXCL10 (data not shown). This indicates that the migratory capacity of B cells not only depends on their expression level of chemokine receptors but also on their state of activation, differentiation and external microenvironment. Furthermore, the inflammatory environment in autoimmune diseases could possibly influence $B$ cell trafficking, rendering them more sensitive to cytokines, which are overexpressed in autoimmune patients. Together, the expression of CXCR3 and CXCR5 on DN B cells, the in vitro migration capacity of MS DN B cells towards CXCL13 and CXCL10 and the elevated frequencies of DN B cells in MS CSF (68) suggest that DN B cells can migrate towards the CSF and thus could exert their pro-inflammatory functions locally inside the CNS of MS patients.

At this moment, there are contradictory results concerning the activation potential of DN B cells. One of the hypotheses of how DN B cells arise is that they are exhausted memory $B$ cells that exhibit downregulated CD27 following chronic antigen stimulation, disabling them to become activated $(352,353)$. For instance, Colonna-Romano et al. showed that DN B cells of HC did not proliferate following CpG2006 stimulation for 5 days (88). Furthermore, Buffa et al. concluded that DN $B$ cells are exhausted cells since they were not activated by anti-CD40/IL-4 or CpG2006 (354). However, in both studies the activation profile of DN B cells resembled that of CSM $B$ cells. Therefore, we assessed the ability of DN B cells to become activated and proliferate after stimulation with CpG2006. Stimulating DN B cells with CpG2006 for 10 days resulted in the strongest proliferative response and highest expression (MFI) of the activation markers CD80 and CD25 compared to naive, NCSM and CSM B cells. However, more HC and MS patients need to be tested to reach statistical significance. The discrepancy with the studies of Colonna-Romano et al. and Buffa et al. could be due to the use of different cell proliferation dyes or due to the activation period.

Our results are in agreement with Martorana et al., who showed that DN B cells from young donors are strongly activated by CpG2006 stimulation (342), but also 
showed the opposite namely that naive and NCSM B cells have a stronger proliferative response compared to DN B cells (342). This could be due to differences in the activation period (10 days vs 3 days) and indicates that $D N$ and CSM B cells require a longer activation period. Furthermore, similar to our results, Wei et al. indicated a positive proliferative response of DN B cells after CpG2006 stimulation which was higher than that of naive B cells (70). Even though there are contradictory results concerning the activation potential of DN B cells, we showed a strong activation of DN B cells following CpG2006 stimulation. These findings indicate that DN B cells are no terminally differentiated cells.

One of the limitations of this study is the lack of comparing the phenotype of different DN B cell subtypes (DN $\mathrm{IgG}^{+}, \mathrm{DN} \mathrm{IgM}^{+}, \mathrm{DN} \mathrm{CD}^{+} 5^{+}$). This should be investigated in future studies because these subtypes could have arisen from a different differentiation pathway and possibly show different functions in the pathology of MS. Furthermore, a more detailed analysis of different DN B cell subtypes and their possible correlation with clinical characteristics of MS patients would reveal which subtypes influence disease progression.

In conclusion, our results indicate that DN B cells show a mature and memory phenotype. However, DN B cells show earlier maturation features compared with CSM B cells. Thus, DN B cells may represent post-germinal center B cells. Furthermore, DN B cells express activation markers and respond to in vitro activation meaning these $B$ cells do not appear anergic or exhausted. Furthermore, expression of pro-inflammatory adhesion molecules and chemokine receptors on DN $B$ cells guides these cells to the site of inflammation where they may contribute to immunopathology. Given the unique phenotype of DN B cells, these cells have the potential to be targeted by biologics, which may prove efficacious in autoimmune diseases. 


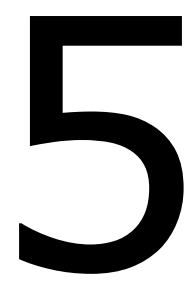

\section{Summary and general discussion}

Partly based on:

Dimethyl fumarate treatment in multiple sclerosis: recent advances in clinical and immunological studies

Montes Diaz G., Hupperts R., Fraussen J.*, Somers V.*

Autoimmunity reviews, 2018

* Equally contributing authors

Phenotypic and Ig Repertoire Analyses Indicate a Common Origin of IgD(-)CD27(-) Double Negative B Cells in Healthy Individuals and Multiple Sclerosis Patients.

Fraussen J, Marquez S, Takata K, Beckers L, Montes Diaz G, Zografou C, Van Wijmeersch B, Villar LM, O'Connor KC, Kleinstein SH, Somers V

J Immunol. 2019 


\section{Summary and general discussion}

Multiple sclerosis (MS) is a chronic inflammatory autoimmune disease of the central nervous system (CNS) in which demyelination and neurodegeneration occurs. The immune system of MS patients is characterized by a dysregulation in the balance between pro- and anti-inflammatory immune cells, whereby both the innate and adaptive immune system are involved. The disease affects approximately 2.3 million people worldwide, primarily affects young adults and until now, no cure is available (1-3). Although there is no cure for the disease, over the last decade the long-term prognosis of patients with relapsing forms of the disease has improved considerably. This is largely due to the advent and increased number of new and highly effective therapies for MS compared to a decade ago (355). Current approved disease-modifying therapies are immunomodulatory or immunosuppressive drugs that regulate or inhibit immunological responses to unknown disease target(s) in the CNS and thus reduce the frequency of attacks of the relapsing forms of the disease (356). However, we have at best a limited understanding of how current therapies alter MS pathophysiology and if MS therapy can restore failed immune tolerance without impeding other parts of the immune system (355). The emergence of multiple treatment options for MS has been accompanied by a complex array of efficacy, adverse events, tolerability and sustainability. In order to make a wellbalanced decision in the treatment plan, an extensive knowledge of each individual treatment's mechanism of action and potential side effects is necessary. Furthermore, discovering the mechanism of action of MS treatments will provide more insight into the role of the different immune cell subtypes in MS pathology. In this thesis, we investigated how dimethyl fumarate (DMF) treatment affected the distribution and function of immune cell populations and studied the phenotype and function IgD-CD27- double negative (DN) B cells in MS patients. In this chapter, the key findings of this thesis are discussed.

DMF treatment persistently changes the immune balance of MS patients and has a multifactorial working mechanism

Multiple treatment options for MS exist, of which DMF is one of the first oral immunomodulating treatments in RRMS and worldwide the most used MS drug. However, its therapeutic mechanism of action has not been fully elucidated before 
entering the market. Longitudinal effects of DMF treatment on different immune cell populations of MS patients were understudied at the time of initiation of this study. Chapter 2 describes the longitudinal effects of DMF treatment on innate and adaptive immune cell populations.

\section{How does DMF treatment affect clinical parameters of MS patients?}

To assure the relevance of the immunological characterization, we took clinical parameters into account. In this study, clinical parameters were analyzed in the $12 \mathrm{~m}$ follow-up study of DMF-treated RRMS patients. Adherence to DMF was previously shown to be affected by its adverse event profile, treatment satisfaction (such as effectiveness) and patient specific factors (such as fatigue, cognition and motor skills) (357). Only two MS patients dropped out of our study due to persistent side effects such as gastrointestinal problems and flushing, indicating that in general DMF is well-tolerated. This is in line with the long-term follow-up data from the ENDORSE study which showed a favorable risk-benefit profile for DMF (286). Furthermore, Aungst et al. demonstrated that a lower concern with experienced side effects correlated with higher adherence rates (357). When considering neuroradiological efficacy, we demonstrated that 4 of the $18 \mathrm{MS}$ patients showed a reduction in the volume of the brain lesions or the brain lesions were less pronounced compared to baseline. The remaining MS patients (14/18) showed a stable disease course, with no new or enlarged lesions. These data demonstrate a beneficial neuroradiological efficacy which is in line with the DEFINE and CONFIRM study (285). In addition, DMF treatment improved cognitive functions namely auditory processing speed and attention (paced auditory serial addition test) of treated MS patients. Motor skills (timed 25-foot walk and 9-hole peg test), patient reported motor fatigue and cognitive fatigue (fatigue scale for motor and cognitive functions (FSMC), a 20-item questionnaire for motor and cognitive functions) remained stable over the course of the study.

Cognitive decline is a serious complication in MS. Aungst et al. demonstrated that adherence to DMF significantly increased when MS symptoms such as memory trouble were relieved (357). The improvement in cognitive function could be the result of the inhibition of the pro-inflammatory immune cells or of the neuroprotective effect of DMF (317). This finding indicates that DMF treatment 
may protect against cognitive decline and a decline in function in MS patients. However, this needs to be validated in a larger cohort of DMF treated MS patients.

\section{How does DMF treatment affect the distribution of immune cell populations in MS patients?}

DMF treatment reduces the absolute number of lymphocytes, $T$ cells and $B$ cells and preferentially reduces cytotoxic $T$ cells

By performing an extensive immunophenotypic analysis of the innate and adaptive immune system of DMF-treated RRMS patients via a longitudinal assay design, we showed decreased absolute numbers of total lymphocytes, $T$ cells and B cells, while monocytes, neutrophils and anti-inflammatory subtypes such as regulatory NK cells and transitional B cells were not affected at $12 \mathrm{~m}$ of treatment. These results suggest that DMF treatment selectively targets or has a stronger effect on particular immune cell subtypes and particularly targets the adaptive immune system. In addition, the change in the composition of the immune cells was persistent after $12 \mathrm{~m}$. Since DMF decreases the total number of lymphocytes, development of prolonged lymphopenia, which is a risk factor for progressive multifocal leukoencephalopathy (PML), should be taken into consideration. DMFassociated $\mathrm{PML}$ is a rare event and previous studies reported an incidence of approximately $1 / 50,000$. To date, a total of six cases of PML were reported in MS patients treated with DMF (358-360). Monitoring the absolute lymphocyte number could reduce the risk for developing prolonged lymphopenia. Furthermore, we have shown that DMF treatment is fully effective on immune cells after $6 \mathrm{~m}$ of treatment, and after $12 \mathrm{~m}$ of DMF treatment the absolute lymphocyte number plateaued (361). This indicates that DMF-treated MS patients with a higher risk of subsequently developing lymphopenia can be identified in the first year of treatment (362). Furthermore, we showed a preferential loss of $\mathrm{CD}^{+} \mathrm{T}$ cells versus $C D 4^{+} \mathrm{T}$ cells.

This reduction of $\mathrm{CD}^{+} \mathrm{T}$ cells is a general characteristic of DMF treatment since it was also detected in psoriasis patients treated with Fumaderm (363).

In the study of Fleisher et al., an increased $\mathrm{CD}^{+} / \mathrm{CD}^{+} \mathrm{T}$ cell ratio following $\mathrm{DMF}$ treatment was associated with stable disease (364). Moreover, the numbers of $\mathrm{CD}^{+} \mathrm{T}$ cells but also of lymphocytes and $\mathrm{B}$ cells were indicated as predictors of 
treatment response (364). Recently, Ciolac et al. showed that DMF-treated MS patients free of disease activity presented with a lower $\mathrm{CD}^{+} \mathrm{T}$ cell number (365). Hereby, the stronger depletion of $\mathrm{CD}^{+} \mathrm{T}$ cells was associated with a more favorable response in terms of cortical integrity and gray and white matter network responses (365).

Since CD8 ${ }^{+} \mathrm{T}$ cells are critical for cell-mediated antiviral immunity, a decrease in $\mathrm{CD}^{+} \mathrm{T}$ cells could result in more prevalent viral infections. In this light, it was suggested that a sustained reduction of $C D 8^{+} \mathrm{T}$ cells could be a risk factor for $\mathrm{PML}$, which is caused by an infection with the JC virus $(302,303)$. However, we and others have not found an increased incidence of serious or opportunistic infections (366). In addition, Metha et al. showed in the long-term DMF clinical study (ENDORSE) with 2,513 patients that the absolute lymphocyte numbers are closely correlated with $\mathrm{CD}^{+} \mathrm{T}$ cell numbers, indicating that lymphocyte subtype monitoring is not required for safety vigilance (366). In conclusion, monitoring the absolute lymphocyte numbers remains the most effective way of identifying patients at risk of developing lymphopenia in DMF-treated MS patients.

DMF treatment preferentially reduces memory and effector memory $T$ and $B$ cells

Further refined immunophenotyping of the major lymphocyte subtypes showed a decrease in the absolute numbers of pro-inflammatory memory $\mathrm{CD}^{+}$and $\mathrm{CD}^{+} \mathrm{T}$ cells, memory B cells (class-switched and non class-switched) and DN B cells after $12 \mathrm{~m}$ of DMF treatment. Naive $\mathrm{CD}^{+}$and $\mathrm{CD}^{+} \mathrm{T}$ cells, naive and transitional $\mathrm{B}$ cells, that are not involved in MS pathology, were unchanged following $12 \mathrm{~m}$ of DMF treatment. Besides, Li et al. showed that already after $3 \mathrm{~m}$ of DMF treatment, mature and differentiated B cells, but not transitional B cells, were preferentially lost in the circulation (295). These data suggest that the output of immature $B$ cells from the bone marrow is not prevented by DMF $(295,316)$.

Next to the absolute numbers, the frequencies of the immune cell subtypes were assessed in order to evaluate the relative composition of the remaining lymphocyte repertoire following DMF treatment. We have shown that DMF treatment decreased the frequencies of central memory and effector memory $\mathrm{CD}^{+}$and $\mathrm{CD}^{+} \mathrm{T}$ cells, while naive $\mathrm{CD}^{+}$and $\mathrm{CD}^{+} \mathrm{T}$ cell frequencies were increased in DMF-treated MS patients. In line with our results, Hansen et al. 
showed a preferential loss of memory T cells (367). In addition, Hansen et al. indicated a decrease in $\mathrm{CD} 4^{+} \mathrm{T}$ cells expressing chemokine receptors and adhesion molecules which are involved in the entry of $T$ cells into CNS and showed a reduced frequency of $\mathrm{CD}^{+}{ }^{+} \mathrm{T}$ cell in the CSF of DMF-treated MS patients compared to untreated MS patients (367). Furthermore, DMF treatment exhibited similar effects on $B$ cells by decreasing the frequencies of non class-switched memory $B$ cells, class-switched memory $B$ cells and DN $B$ cells while increasing naive and transitional $B$ cells in MS patients. In conclusion, depletion of memory and effector memory $T$ and $B$ cells is one of the working mechanisms of DMF in MS. In this way, DMF is capable of redirecting the disrupted immune balance of MS patients away from the pro-inflammatory immune response.

\section{DMF treatment inhibits pro-inflammatory cytokine-expressing $T$ cells}

Our studies indicated reduced frequencies of pro-inflammatory GM-CSF${ }^{+}, \mathrm{IFN}^{-} \mathrm{\gamma}^{+}$, and $\mathrm{IL}-17^{+} \mathrm{CD} 4^{+} \mathrm{T}$ cells of DMF-treated MS patients following ex vivo stimulation, while the frequencies of $\mathrm{IL}_{-} 4^{+}$and $\mathrm{IL}-10^{+} \mathrm{CD} 4^{+} \mathrm{T}$ cells remained unchanged. However, Medina et al. could not find an effect of DMF treatment on GM-CSF${ }^{+}$, $\mathrm{TNFa}^{+}$and $\mathrm{IL}_{17} 7^{+} \mathrm{CD} 4^{+} \mathrm{T}$ cells. This could be due to the use of different stimulation conditions and a $6 \mathrm{~m}$ follow-up while our study as well as the study of Wu et al.

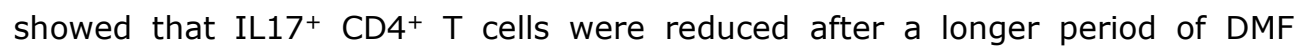
treatment $(304,309,316)$. Due to technical limitations we did not analyze the effect of DMF treatment on the frequency of cytokine expressing CD8 ${ }^{+} \mathrm{T}$ cells. Nonetheless, other studies showed the same trend as seen for cytokineexpressing $\mathrm{CD}^{+}{ }^{+} \mathrm{T}$ cells, namely, that DMF treatment reduced the frequency of pro-inflammatory IFN- $\gamma^{+}$and TNF- $\mathrm{a}^{+} \mathrm{CD}^{+}{ }^{+} \mathrm{T}$ cells while the frequency of IL-10+ $\mathrm{CD}^{+} \mathrm{T}$ cells did not change in MS patients (Figure 5.1) $(301,304,368)$. In addition, reductions of IFN $-\mathrm{y}^{+} \mathrm{CD} 4^{+}$and $\mathrm{CD} 8^{+} \mathrm{T}$ cells and TNF- $\mathrm{a}^{+} \mathrm{CD} 8^{+} \mathrm{T}$ cells could be predictors for stable disease in DMF-treated MS patients (304).

In conclusion, DMF treatment shifts the inflammatory Th1, Th17 and proinflammatory cytokine expressing $\mathrm{CD} 8^{+} \mathrm{T}$ cells to a more tolerogenic response in MS patients. 
DMF treatment decreases the CD56 dim NK cell number and increases the CD56 bright NK cell frequency

When considering different subtypes of NK cells, DMF treatment was shown to decrease the absolute number of CD56 dim NK cells while CD56 bright NK cells were not affected in our follow-up study (Figure 5.1). Further, the frequency of CD56 bright NK cells was increased while the frequency of CD56 dim NK cells were not affected in DMF-treated MS patients after $12 \mathrm{~m}$ of treatment. CD56 ${ }^{\text {bright }}$ NK cells have been considered to be different from CD56 dim NK cells because they showed a reduced cytotoxicity and an enhanced cytokine production (78). The beneficial role of CD56 bright $\mathrm{NK}$ cells in the pathogenesis of MS patients was highlighted when their expansion was correlated with the efficacy of daclizumab, a humanized antiCD25 monoclonal antibody, in MS treatment (306). Since CD56 bright NK cells can lyse autoreactive $\mathrm{T}$ cells, harbor a high migratory capacity across the blood-brain barrier and are enriched in the cerebrospinal fluid of MS patients $(369,370)$, they are potentially important to limit autoimmunity following DMF treatment. Furthermore, the study of Smith et al. demonstrated that NK cells of DMF-treated MS patients presented with an increased cell degranulation and cytotoxicity towards activated autologous T cells when treated with DMF or MMF compared to vehicle (Figure 5.1) (371). Additionally, they showed that the expression of CD155, involved in NK cell cytolysis (369), was preferentially expressed on CD8 ${ }^{+}$ $\mathrm{T}$ cells and central memory $\mathrm{T}$ cells compared to $\mathrm{CD} 4^{+} \mathrm{T}$ cells and naive $\mathrm{T}$ cells, respectively (371). This could point to the involvement of CD56 bright $\mathrm{NK}$ cells in the preferential loss of memory $\mathrm{CD} 8^{+} \mathrm{T}$ cells, which was also suggested by the finding of a negative correlation between the frequency of CD56 bright $\mathrm{NK}$ cells and memory $\mathrm{CD}^{+} \mathrm{T}$ cells $(304,371)$. In conclusion, the restored cytolytic function of NK cells in DMF-treated MS patients could play a role in controlling autoimmunity via preferential targeting of $\mathrm{CD}^{+} \mathrm{T}$ cells and memory $\mathrm{T}$ cells.

\section{B cells as targets of DMF treatment}

We investigated the effect of DMF treatment on different $B$ cell functions. In Chapter 2 we showed that DMF treatment reduces the absolute number of B cells and alters the distribution of several B cell subtypes including a reduction in the percentage of memory $B$ cell subtypes. 
Because of the reduced absolute number of B cells, we investigated if DMF could directly deplete B cells as a result of apoptosis. Since B cells are targeted by DMF treatment, we assessed the influence of longitudinal DMF treatment (Chapter 2) and DMF and MMF in vitro treatment (Chapter $\mathbf{3}$ ) on the expression of survival markers and antigen presentation and costimulatory functions. Secondly, the direct effect of DMF and MMF on cytokine-expressing B cells (Chapter $\mathbf{3}$ ) is discussed.

\section{How does DMF treatment affect the functions of B cells in MS patients?}

DMF treatment induces apoptosis of B cells

We investigated whether the reduction in B cells in DMF-treated MS patients was the result of apoptosis of these cells. We showed that in vitro treatment with DMF, but not MMF, induced apoptosis of polyclonally stimulated B cells from HC and untreated MS patients in a concentration-dependent manner with B cells of MS patients appearing to be more vulnerable. Treumer et al. also showed that DMF but not MMF lowered the expression of $\mathrm{Bcl}-2$ in human T cells (319). This could be due to the fact that DMF is involved in the covalent modifications of proteins, while MMF is a poorly reactive hydrolytic metabolite which does not appreciably react with cysteine residues of proteins $(258,372)$. In addition, it has been shown that DMF reacts faster with cellular GSH than MMF (373). Furthermore, it is indicated that DMF enters the cell and modifies many proteins before being hydrolyzed to the less reactive MMF (374).

Additionally, Li et al. detected a preferential effect of in vitro DMF on the induction of apoptosis in mature B cells compared to transitional B cells of HC (295). Ghadiri et al. showed a similar preference of DMF when considering $T$ cell subtypes. Central memory and effector memory $\mathrm{CD}^{+}$and $\mathrm{CD}^{+} \mathrm{T}$ cells exhibited greater susceptibility to DMF-induced apoptosis than naive T cells and Treg (301). Furthermore, Ghadiri et al. and Wu et al. detected a preferential effect of DMF on apoptosis induction of $\mathrm{CD}^{+} \mathrm{T}$ cells compared to $\mathrm{CD}^{+} \mathrm{T}$ cells of $\mathrm{HC}(301,309)$. One of the molecular mechanisms that could be responsible for the induction of apoptosis by DMF is via downregulation of $\mathrm{Bcl}-2$ expression, a survival protein which is responsible for the inhibition of apoptosis (319). Furthermore, it has been 
shown that DMF can induce apoptosis of T cells via the induction of oxidative stress via depleting GSH and decreasing the levels of antioxidative compounds (275).

In conclusion, our data combined with that of others suggests that DMF but not MMF can directly induce apoptosis in B cells and T cells. The preferential reduction of mature $\mathrm{B}$ cells, $\mathrm{CD} 8^{+} \mathrm{T}$ cells and memory immune cell subtypes detected $\mathrm{ex}$ vivo in DMF-treated MS patients can be the result of their greater susceptibility to DMF-induced apoptosis.

DMF treatment interferes with antibody-independent functions of $B$ cells

In Chapter 2, we showed that DMF treatment did not change the frequency of $\mathrm{IgG}^{+}$or $\mathrm{IgA}^{+} \mathrm{B}$ cells in the peripheral blood of MS patients. Similarly, other studies did not find a change in serum levels of total IgG or IgA $(275,316,368)$. However, for several years now, the antibody-independent functions of $B$ cells are recognized to be increasingly important in the pathology of MS. In Chapter 3, our study demonstrated that DMF directly decreased expression of antigen presentation molecule HLA-DR/DP/DQ, costimulatory molecule CD40 and survival marker BAFFR on B cells of MS patients using in vitro experiments (Figure 5.1). Other studies have shown a decrease in B cell expression of surface markers that are important for B cell - T cell interactions, such as CD11C, CD43, CD80 and CD83, following DMF treatment (Figure 5.1) (295). In addition, polyclonal activation of $B$ cells from DMF-treated MS patients could be eliminated and even reversed by in vitro DMF treatment via decreasing CD40, CD86 and CD150 expression (275). The reduced expression of molecules involved in antigen presentation and $B$ cell activation are thought to be the result of altered phosphorylation pattern in the pathway of NFKB, MAPK and ERK1/2 by DMF and MMF $(275,307)$. Interestingly, after $3 \mathrm{~m}$ of DMF treatment, the ex vivo activation potential of B cells decreased in MS patients compared to baseline (275).

Recently, Traub et al. demonstrated that DMF treatment continuously declined the expression of activation markers CD25, CD69 and CD95, suggesting that DMF dampens the pro-inflammatory B cell activity (375). Furthermore, the same authors found that the CNS of MS patients treated with DMF showed a diminished number of damaged axons and presented with a high number of mature oligodendrocytes (375). In conclusion, DMF downregulates expression of 
functional markers on B cells which are involved in antigen presentation, B cell activation and $\mathrm{T}$ cell interaction. The reduced costimulatory capacity may induce immune hyporesponsiveness, which contributes to the clinical effect and the reduction of the autoimmune response in DMF-treated MS patients.

DMF treatment increases anti-inflammatory Breg and inhibits pro-inflammatory cytokine-expressing $B$ cells in a direct manner

In Chapter 2, the frequency of transitional B cells, that can differentiate into regulatory $B$ cells (Breg), was increased following DMF treatment in MS patients. Using in vitro experiments, we showed in Chapter $\mathbf{3}$ a trend towards an increase in the frequency of Breg of MS patients after DMF treatment. Lundy et al. reinforced this finding and described an increase in the frequency of Breg in DMFtreated MS patients although the extent of the increase varied greatly between patients, a finding that might explain the lack of an effect on Breg in the study of Medina et al. $(304,311)$. Breg suppress Th1 and Th17 cell differentiation while inducing the generation of regulatory $T$ cells (Treg) and could therefore contribute to the efficacy of DMF in MS treatment (328). Furthermore, we showed a trend towards a decrease in pro-inflammatory TNF- $\mathrm{a}^{+} \mathrm{B}$ cells of MS patients after in vitro treatment with MMF. Other studies have shown that DMF treatment decreased the absolute number and frequency of pro-inflammatory TNF- $\mathrm{a}^{+}, \mathrm{GM}^{-\mathrm{CSF}^{+}}$and IL-6 ${ }^{+}$ $B$ cells while no effect was observed on IL-10+ $B$ cells in MS patients $(295,310)$. These data contribute to the DMF induced shift in the immune balance to a more anti-inflammatory profile. 


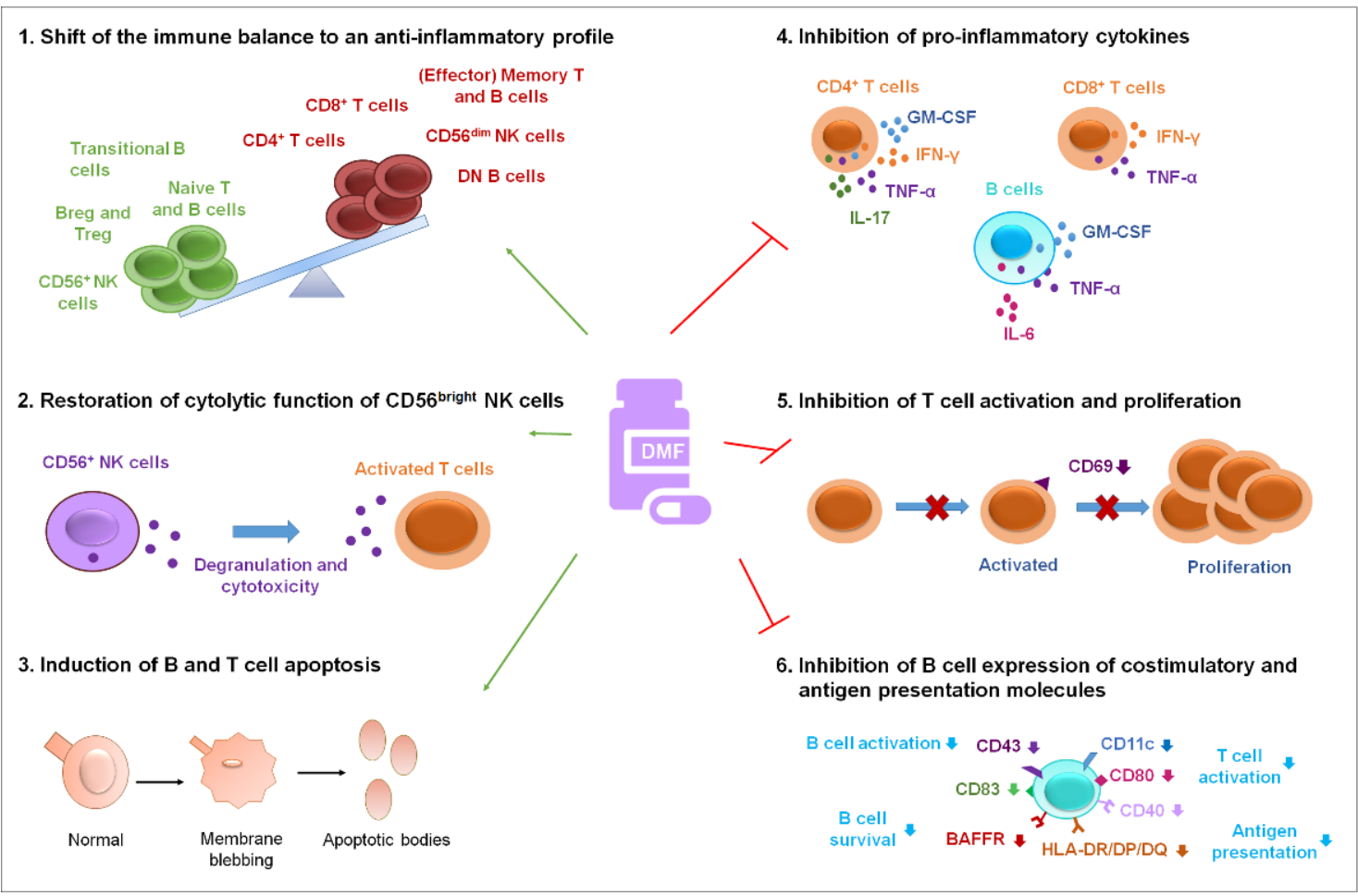

Figure 5.1. Effects of DMF treatment on immune cells of MS patients. (1)

DMF treatment shifts the immune balance of MS patients from a pro-inflammatory $\left(\mathrm{CD} 4^{+} \mathrm{T}\right.$ cells, CD8 ${ }^{+} \mathrm{T}$ cells, effector memory $\mathrm{T}$ and $\mathrm{B}$ cells, DN B cells, CD56 ${ }^{\mathrm{dim}} \mathrm{NK}$ cells) to an antiinflammatory profile (naive $T$ and B cells, Breg and Treg, CD56 bright NK cells, transitional B cells). (2) DMF treatment restores the cytolytic function of CD56 bright NK cells towards activated $T$ cells, which results in increased degranulation and cytotoxicity. (3) DMF treatment induces apoptosis of $T$ and B cells. (4) DMF treatment inhibits the expression of pro-inflammatory cytokines by $\mathrm{CD}^{+} \mathrm{T}$ cells (GM-CSF, IFN- $\gamma, \mathrm{TNF}-\mathrm{a}, \mathrm{IL}-17$ ), CD8 ${ }^{+} \mathrm{T}$ cells (IFN- $y$, TNF-a) and B cells (GM-CSF, TNF-a, IL-6). (5) DMF treatment inhibits T cell activation by reducing CD69 expression and inhibits $T$ cell proliferation. (6) DMF inhibits $B$ cell expression of surface markers important for B cell - T cell interaction (CD11C, CD43, CD80, CD83), of costimulatory molecule CD40, of antigen presentation molecule HLA$\mathrm{DR} / \mathrm{DP} / \mathrm{DQ}$ and of survival marker BAFFR. DMF = dimethyl fumarate, GM-CSF = granulocytemacrophage colony-stimulating factor, IFN- $\gamma=$ interferon $-\gamma$, TNF- $a=$ tumor necrosis factor alpha, BAFFR = B-cell activating factor receptor. 


\section{DMF treatment compared to other MS treatments}

DMF treatment is characterized by pleiotropic biological effects, meaning that DMF does not exert a single mechanism of action. DMF is a clear immunomodulator with the ability to promote a polarized shift away from a pro-inflammatory state by altering both $\mathrm{T}$ and $\mathrm{B}$ cell subtype composition and cytokine expression. The reason for these pleiotropic effects of DMF treatment is that it interacts directly with multiple proteins (e.g.: transcription factors, proteins of NF-kb signaling pathway, receptors and free cysteines), which leads to multiple functional changes such as induction of apoptosis, antioxidant properties, anti-inflammatory activity, inhibition of pro-inflammatory cytokines and change in immune cell distribution (317). Because of this direct interaction with multiple proteins, DMF is different from other MS treatments such as teriflunomide, fingolimod and natalizumab, which are designed to target one protein (376). Furthermore, the working mechanism of DMF differs from that of cladribine, alemtuzumab and ocrelizumab since DMF has a more immunomodulating effect and interferes with different functions of immune cells. Cladribine is designed to have a selective cytotoxic activity towards $\mathrm{B}$ cells, $\mathrm{CD}^{+}$and $\mathrm{CD}^{+} \mathrm{T}$ cells, while alemtuzumab depletes lymphocytes expressing CD52 ( $T$ cells and B cells) by antibody-dependent, cellmediated and complement-dependent cytolysis $(377,378)$. Furthermore, ocrelizumab selectively depletes CD20- expressing immature and mature B cells (378). The working mechanism of IFN- $B$ treatment is similar to DMF since IFN- $\beta$ also activates multiple signal-transduction pathways that result in immunomodulatory and antiproliferative effects. However, IFN- $\beta$ is a cytokine that evokes the production of antidrug antibodies, reducing treatment efficacy (378). GA's main working mechanism is the induction of MBP-related $T$ cell immune responses which results in promoting regulatory $T$ cells. Similar to DMF, GA reduces the number of memory $B$ cells, but it also shifts the immune response to an anti-inflammatory $B$ cell phenotype. However, the working mechanisms behind these results are different from DMF as these are the result of binding of GA with $\mathrm{T}$ and $\mathrm{B}$ cell receptors (378). In addition, $\mathrm{DMF}$ has demonstrated relative improvement in real-world effectiveness versus first-line agents such as IFN- $\beta$, GA and teriflunomide (379). In conclusion, DMF has an unique pleotropic working mechanism which interferes with multiple immune cell phenotypes and functions resulting a shift away from a pro-inflammatory state. 


\section{DN B cells as targets in the pathogenesis of MS}

In Chapter 2, we observed a decrease in the percentage of memory and DN B cells in MS patients treated with DMF. At the moment, not much is known about DN B cells and definitely not about their role in MS. DN B cells could be important in MS pathology due to the clinical success of DMF and B cell depleting treatments.

DN B cells are increased in MS patients and previous work from our group demonstrated that DN B cells exhibited a pro-inflammatory profile. Therefore, we aimed to unravel the developmental and migratory phenotype and functions of DN B cells in the pathology of MS in more detail (Chapter 4).

\section{What is the phenotype of DN B cells in MS patients and HC?}

$D N B$ cells of MS patients and HC show a phenotype that resembles that of CSM $B$ cells, however in an earlier maturation state

DN B cells have an expression profile of developmental markers more similar to CSM than naive $B$ cells and our results demonstrated that the majority of DN B cells are mature antigen-experienced B cells. Their mature state was indicated by the low expression of CD38 and the immature markers CD5 and CD10 (Chapter 4). In SLE patients, the majority of DN B cells also showed a low expression of

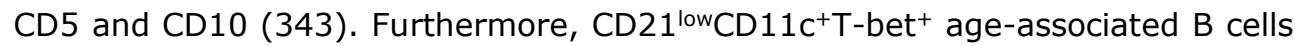
$(339,380)$ and DN B cells in SLE $(381)$ are similarly characterized by the absence of CD38. DN B cells of MS patients are antigen-experienced since the majority of DN B cells was made up by $\mathrm{IgG}^{+}$and $\mathrm{IgA}^{+}$cells (Chapter 4 ), which is in agreement with previous results (68) of our research group and with studies in aged HC (90, 354 ) and RA patients (161). However, the frequency of IgA ${ }^{+}$cells was lower in DN $B$ cells compared to CSM B cells (Chapter 4 ), which is in line with findings in HC (382) and RA patients (161). Nonetheless, a proportion of DN B cells probably has an activated phenotype since the frequency of CD95 ${ }^{+}$cells within the DN B cell population was higher compared to naive B cells, nonetheless lower compared to CSM B cells. Since in SLE patients, CD95+ DN B cells correlated with disease activity and increased with disease flares (343), it would be interesting to investigate if $\mathrm{CD}^{+} 5^{+}$DN B cells are also correlated with disease activity and clinical characteristics (EDSS, relapse rate, fatigue -, cognition- and motor function and MRI results) of MS patients. Taken together, the intermediate frequency of $\mathrm{CD}^{+}$, 
$\mathrm{CD} 8^{+}$, and $\mathrm{CD}^{+} 5^{+}$cells in the DN B cell population between that of the naive and CSM B cell populations could point to an intermediate developmental state.

To further investigate the origin and selection characteristics of DN B cells, our research group performed high-throughput adaptive immune receptor repertoire sequencing of DN and CSM B cells from HC and MS patients ( $n=3$ each) (383). DN B cells from MS patients and HC showed similar Ig repertoire characteristics, indicating a common developmental pathway. $\mathrm{IgG}^{+} \mathrm{DN}$ B cells were more related to CSM B cells, although DN B cell Ig repertoires showed a lower mutation load compared to those of CSM B cells. IgM+ DN B cells showed even less mutations in the Ig VH genes and showed differences in V, D, and J family usage, indicating that DN B cell subpopulations may utilize unique differentiation pathways or respond to different antigenic stimuli (383). This suggests that the DN B cell population consists of $B$ cells with different origins dependent on the isotype. Furthermore, as observed in Chapter 4, the majority of DN B cells showed earlier maturation features compared to CSM B cells and could be formed via differential activation pathways. However, a small part of DN B cells are clonally related to CSM B cells and could be exhausted memory B cells (383). Additionally, classswitched CD27- B cells showed lower Ig mutation frequencies compared to $\mathrm{CD} 27^{+}$ $B$ cells. As isotype switching occurs before somatic hypermutation during the germinal center response, it was proposed that class-switched DN B cells could be involved in (primary) $\mathrm{T}$ cell dependent immune responses and prematurely exit the germinal center response (383).

DN B cells of MS patients and HC do not differ in the expression of developmental markers and Ig repertoires

We report the absence of major differences in both the developmental marker expression (this thesis) and the Ig repertoires (383) of DN B cells in HC and MS patients. This leads us to suggest that these cells have a similar origin in both cohorts. As mentioned by Fraussen et al. (383) elevated frequencies of DN B cells could be triggered because of the aging process that is common in the immune system of the elderly (384) and occurs prematurely in a proportion of MS patients (144). Aging of the immune system is accompanied by a low-grade chronic inflammation, termed "inflammaging," that is characterized by an increase in proinflammatory cytokines (IL-6, IL-15, IL-8), an increase in other inflammatory 
mediators (such as coagulation factors), and subclinical infections with common viruses (385). This chronic inflammatory environment combined with genetic predisposition could result in the expansion of DN B cells in aged individuals and a proportion of MS patients. Furthermore, a possible aberrant germinal center response in MS patients and in aged individuals $(151,386,387)$ could be another reason for the expansion of DN $B$ cells in these two cohorts. In conclusion, developmental surface marker expression and Ig repertoire characteristics suggest that DN B cells share a common developmental pathway in HC and MS patients that leads to expansion of these cells because of inflammaging.

About $23 \%$ of DN B cells express T-bet in MS and HC

T-bet is a master regulator of the $\mathrm{CD} 11 \mathrm{C}^{+}$age-associated $\mathrm{B}$ cell character in mice and its expression is required for class switching to IgG2a (IgG1 in human) and the production of pathogenic autoantibodies in $\mathrm{B}$ cells $(178,387,388)$. In SLE, Tbet $^{+} B$ cells have autoreactive $B$ cell receptors and are prone to differentiate into IgG autoantibody producing plasmablasts (339). CD $11 \mathrm{C}^{+} \mathrm{CD} 21^{\text {low }} \mathrm{B}$ cells (CD21 low $)$ are the human equivalent of the $C D 11 C^{+}$age-associated $B$ cells described in mice (178). In Chapter 4, we investigated if CD21 low B cells and DN B cells of MS patients express T-bet. We demonstrated that about $23 \%$ of DN B cells express T-bet, while about $70 \%$ of $C D 21^{\text {low }} \mathrm{B}$ cells express T-bet in MS patients. This confirms that $C D 21^{\text {low }} B$ cells are most likely the human equivalent of T-bet ${ }^{+}$ageassociated $B$ cells found in mice $(172,339)$. However, this also indicates that the subtype of T-bet ${ }^{+}$DN B cells may resemble the T-bet driven CD $21^{\text {low }} B$ cells and therefore can possibly exert different functions in the pathology of MS compared to other subtypes of DN B cells. Recently, DN B cell expansions in SLE were assigned to a subtype of CXCR5-CD21-CD11 ${ }^{-}{ }^{-}$T-bet ${ }^{+}$cells (381). These "DN2" cells differentiated into autoantibody-producing plasma cells driven by toll-like receptor 7, which led to their characterization as extrafollicular $B$ cells responding to innate stimuli. As previously shown by our research group, $\sim 20 \%$ of DN B cells could be retraced to the $C D 21^{\text {low }} C D 11 C^{+} B$ cell gate (68). Therefore, we expect that the majority of DN B cells in MS patients are not DN2 cells. As mentioned in Fraussen et al., this could be explained by the difference in pathological mechanisms in SLE and MS that center around antibody-mediated and cell-mediated immune mechanisms, respectively. Moreover, a subtype of DN B cells with cytoplasmic 
FOX01, a transcription factor involved in B cell development, was found to be increased in SLE patients and positively correlated with disease activity (389). Whether this DN B cell subtype corresponds with DN2 cells is not clear (383). Involvement of the T-bet-driven pathway in the abnormal elevations of DN B cells in MS remains to be determined.

\section{Can DN B cells contribute to the pathogenesis of MS?}

DN B cells of MS patients express adhesion molecules and chemokine receptors and have the capacity to migrate towards CXCL13 and CXCL10 in vitro

In previous research of our group, an increased percentage of DN B cells in the CSF of MS patients compared with paired peripheral blood samples was found (68). Moreover, DN B cells were shown to be able to migrate from the CNS to the periphery and vice versa since similar DN B cell clones were found in the CSF and peripheral blood of MS patients (91). In order to explain this expansion in the CSF, the expression of adhesion molecules and the migration profile of DN B cells was investigated in Chapter 4. DN B cells were shown to have a pro-migratory chemokine receptor expression pattern and an intermediate expression of adhesion molecules (LFA-1, VLA-4 and ALCAM) in between naive and CSM B cells. Besides the expression of chemokine receptors, we showed in Chapter 4 that DN $B$ cells of MS patients have the capacity to migrate towards the pro-inflammatory chemokines CXCL13 and CXCL10 with an efficacy comparable to that of CSM B cells. Recently, it has been shown that B cells expressing CXCR3 were selectively enriched in CSF, meninges and brain tissue compartments compared to blood of MS patients (390). Furthermore, IFN-y and pathogen-associated TLR9 stimulation induced T-bet expression in B cells of MS patients and this led to an increase in CXCR3 expression on $B$ cells, probably underlying their selective recruitment to the CNS of MS patients (390). In addition, CXCR3+T-bet ${ }^{\text {high }} \mathrm{B}$ cells showed increased IgG1 secretion which implicated that after preferential recruitment and reactivation in the CNS, CXCR3 ${ }^{+}$- -bet $^{\text {high }} \mathrm{B}$ cells are responsible for local production of IgG1 in MS (390). Since our study demonstrated that a proportion of DN B cells express T-bet and CXCR3, the study of Langelaar et al. reinforces a possible pathological role for $\mathrm{CXCR} 3^{+} \mathrm{T}$-bet ${ }^{\text {high }}$ DN $\mathrm{B}$ cells in mediating CNS pathology in MS since CXCR3 ${ }^{+}$T-bet ${ }^{\text {high }}$ DN B cells can be part of the CXCR3 ${ }^{+} \mathrm{T}^{-}$ bet $^{\text {high }} B$ cell population. Since our research indicates that DN B cell have the 
possibility to migrate towards pro-inflammatory chemokines, which are present in the CNS, a next step would be to investigate the presence of DN B cells in brain lesions of MS patients.

DN B cells of HC can become activated following TLR9 stimulation

Since contradictory results exist on the proliferation and activation potential of DN B cells, we investigated in Chapter 4 if DN B cells are able to proliferate and if they can get activated. Although not significant due to the low number of included subjects, after TLR9 stimulation for 10 days, DN B cells of HC showed the strongest proliferative response and highest expression of activation markers (CD80 and CD25) compared to naive, NCSM and CSM B cells. This indicates that DN B cells are not completely exhausted. In conclusion, DN B cells are no terminally differentiated cells.

Possible contribution of DN B cells to the pathogenesis of MS

In general, DN B cells are abnormally elevated in the peripheral blood and CSF of MS patients (68). The expansion of DN B cells in MS is probably due to the stimulating inflammatory environment as seen in aged individuals. Our result suggest that DN B cells have a high migration capacity towards pro-inflammatory chemokines and previous results have shown that DN B cells are found in the CSF of MS patients. This means that in MS, DN B cells can enter the CNS and play a role in MS pathology by contributing in the damage by their pro-inflammatory and cytotoxic cytokine profile (68), their potential to induce T cell responses (68), their activated and mature antigen-experienced phenotype (383). Our group recently showed that a subtype of DN B cells could be autoreactive since an increase in IGHV4-34 gene segments and longer CDR3 length of the Ig repertoire of DN B cells of MS patients was observed when compared with CSM B cells (383).

Although DN B cells showed an intermediate developmental phenotype between naive and CSM B cells, DN B could play a role in the pathogenesis of MS since the majority of DN B cells express $\mathrm{IgG}^{+}$and since they show potential to be autoreactive. Another indication that DN B cells are involved in the pathogenesis of MS is shown by the study of Eggers et al. where the percentage of DN B cells in peripheral blood was positive correlated with gadolinium-enhancing brain lesions implicating that DN B cells are increased in active MS (391). However, our 
research showed that it is important to consider subtypes of DN B cells when further investigating their role in the pathogenesis of MS. Furthermore, there is no evidence yet about their presence in the lesions of MS patients. In order to further understand their role in the pathogenesis of MS, a next step is to correlate the percentage of DN B cells with clinical characteristics (EDSS, relapse rate, fatigue -, cognition- and motor function and MRI results) (391).

\section{Concluding remarks}

The results obtained in this thesis demonstrate that the mechanism behind the beneficial clinical effect of DMF in MS patients is multifactorial. In general, DMF has an immune modulating effect shifting the disturbed immune balance of MS patients away from the pro-inflammatory profile. DMF treatment selectively reduces $\mathrm{CD}^{+} \mathrm{T}$ cells and inflammatory memory subtypes of $\mathrm{T}$ and $\mathrm{B}$ cells in MS patients. Furthermore, pro-inflammatory cytokine expression or production by $\mathrm{T}$ cells and $B$ cells is reduced in DMF-treated MS patients, shifting the Th1/Th17 and pro-inflammatory B cell response to an anti-inflammatory response. Functionally, DMF treatment induces apoptosis of $B$ cells and reduces the expression of survival, antigen presentation and costimulatory markers on B cells. In conclusion, DMF treatment can reduce the autoimmune processes in MS pathogenesis via inhibiting the pro-inflammatory immune cells by interfering with the molecular mechanisms responsible for their harmful functions and by enhancing regulatory immune cells.

DMF treatment decreased the percentage of DN B cells in MS patients. DN B cells are age-associated B cells with a pro-inflammatory functional profile and are increased in MS patients. In this thesis, we showed that DN B cells are mature antigen-experienced $B$ cells resembling CSM B cells in a less advanced developmental state. Furthermore, different subtypes of DN B cells exist, which indicates that different functions can be exerted by DN B cells in MS patients. There is a strong indication that DN B cells can contribute to the pathogenesis of MS since DN B cells have a higher migratory capacity towards pro-inflammatory chemokines involved in B cell trafficking to the CNS in MS. Moreover, DN B cells can proliferate and become activated following TLR9 stimulation. Further study is warranted to examine the downstream pathways that lead to increased frequencies of DN B cells in a proportion of MS patients and whether DN B cells play a role in MS pathology. 
Nederlandse samenvatting 


\section{Nederlandse samenvatting}

\subsection{Wat is multiple sclerose?}

Multiple sclerose (MS) is een chronische auto-immuunziekte, waarbij de eigen immuuncellen het centraal zenuwstelsel beschadigen door myeline af te breken in een proces dat demyelinisatie genoemd wordt. Het myeline beschermt de neuronen en ondersteunt een versnelde impulsgeleiding als isolerende laag rond de axonen. Wanneer myeline wordt afgebroken ten gevolge van MS wordt de signaalgeleiding tussen neuronen verstoord en treden symptomen op zoals vermoeidheid, gevoelloosheid in de ledematen, oogzenuw ontsteking, verminderde coördinatie en cognitieve stoornissen, maar ook verlammingsverschijnselen.

Het klinisch ziektebeeld van MS kan gecategoriseerd worden als ofwel primair progressieve MS (PPMS), relapsing-remitting MS (RRMS), en secundair progressieve MS (SPMS). Patiënten met PPMS hebben een ziekteverloop dat vanaf de start van de ziekte progressief verergert zonder periodes van herstel, met onherstelbare schade tot gevolg. Het overgrote deel van de MS patiëntenpopulatie (85-90\%) wordt gediagnosticeerd met RRMS, waarbij periodes van opstoten (relapses) worden afgewisseld met periodes van gedeeltelijk herstel (remissie). Het ziekteverloop van patiënten met RRMS kan na verloop van tijd overgaan naar de SPMS vorm, waarbij de ziekte progressief verergert zonder periodes van herstel.

De diagnose van MS wordt gebaseerd op klinische vervolg, het voorkomen van een tweede opstoot en een neurologisch onderzoek, waarbij laesies (plaques) in de hersenen gevisualiseerd worden door medische beeldvorming (magnetic resonance imaging, MRI). MS wordt in het algemeen gediagnosticeerd bij jonge mensen met een leeftijd van 20 tot 40 jaar, en dit driemaal vaker bij vrouwen dan bij mannen.

De oorzaak van MS is niet geheel gekend, maar lijkt vrij complex omdat meerdere factoren kunnen een rol spelen bij de ontwikkeling van de ziekte, zoals genetica en omgevingsfactoren. Het immuunsysteem beschikt over mechanismen die ervoor zorgen dat lichaamseigen componenten niet worden aangevallen. Dit wordt tolerantie genoemd. In MS werken deze mechanismen niet meer zo efficiënt, 
waardoor er een auto-immuun respons ontstaat waarbij auto-reactieve immuuncellen zoals T- en B-cellen geactiveerd geraken en het myeline aanvallen.

\subsection{Welke immuuncellen spelen een rol bij MS?}

De pathogenese van MS wordt gekarakteriseerd door een verstoorde balans in het immuunsysteem, waarbij er meer pro-inflammatoire immuuncellen aanwezig zijn dan anti-inflammatoire immuuncellen. Het menselijke immuunsysteem bestaat uit een zogenaamde aangeboren en een verworven component. Het aangeboren immuunsysteem bestaan uit cellen (zoals bijvoorbeeld monocyten, granulocyten, natural killer cellen) die instaan voor de snelle en aspecifieke respons tegen lichaamsvreemde cellen in het lichaam. Daarentegen bestaat het verworven immuunsysteem uit lymfocyten ( $T$ - en B-cellen), die een vertraagde immuunrespons uitvoeren die meer specifiek is tegen lichaamsvreemde cellen. Historisch gezien werden T-cellen beschouwd als de belangrijkste immuuncellen in het ziekteproces van MS. De laatste jaren werd echter duidelijk dat zowel T-als B-cellen hierin een centrale rol spelen.

De B-cel en T-cel populatie bestaat uit verschillende subtypes van cellen, die niet allemaal betrokken zijn bij de pathogenese van MS. Naïeve B- en T-cellen zijn lymfocyten die circuleren door het lichaam, maar eerder nog niet geactiveerd werden. Eens geactiveerd, zullen deze cellen vermeerderen in aantal en krijgen ze meer specifieke functies, waardoor geheugen cellen en effector cellen gevormd worden. Het zijn deze geheugen en effector cellen die van belang zijn in de ziekteprocessen van MS. De T-cel populatie kan onderverdeeld worden in CD4+ helper T-cellen (Th cellen) en $\mathrm{CD}^{+}$cytotoxische T-cellen. Subtypes van de Th cellen, zoals Th1 en Th17 cellen, verergeren het ziektebeeld van MS door de productie van interferon-y (IFN-y) en interleukine 17 (IL-17), die beide moleculen zijn die zorgen voor ontstekingsreacties. Cytotoxische $\mathrm{CD} 8^{+} \mathrm{T}$-cellen verergeren ook het ziektebeeld van MS door de productie van moleculen zoals granzyme B en perforine, die zorgen voor ontstekingsreacties en cytotoxiciteit (doden van cellen). Geheugen B-cellen $\left(\mathrm{CD} 27^{+}\right)$worden beschouwd als het belangrijkste Bcel subtype in het ziektebeeld van MS omdat ze pro-inflammatoire cytokines en antilichamen produceren, en omdat ze T-cellen activeren via co-stimulatie en antigeen presentatie. Naast geheugen B-cellen worden dubbel negatieve B-cellen (IgD-CD27; DN B-cellen) meer recentelijk eveneens aangeduid als mogelijke 
belangrijke speler in het ziekteverloop van MS. In het gezonde lichaam wordt een pro-inflammatoire respons steeds beperkt gehouden door een anti-inflammatoire respons die de ontsteking kan verminderen en kan doen stoppen. Deze antiinflammatoire respons is eveneens belangrijk voor het voorkomen van autoimmuunziekten. Anti-inflammatoire immuuncellen zoals regulatoire T- (Treg) en B-cellen en regulatoire NK-cellen produceren minder anti-inflammatoire cytokines en kunnen auto-reactieve T- en B-cellen minder efficiënt onderdrukken in MS. Dit versterkt de verstoorde balans nog verder.

Naast het verworven immuunsysteem kan ook het aangeboren immuunsysteem, bestaande uit natural killer (NK) cellen en monocyten, een rol spelen in het ziektebeeld van MS.

\subsection{Welke behandelingen zijn er voor MS?}

De eerste therapie voor MS, namelijk interferon- $\beta$, werd ontwikkeld in 1993. Dit was voor lange tijd de enige behandeling beschikbaar voor MS-patiënten. Momenteel zijn er twaalf behandelingen voor handen die goedgekeurd zijn door de European Medicines Agency voor de behandeling van MS. De huidige therapieën zijn ofwel immunomodulerend ofwel immunosuppressief. Eerstelijnsbehandelingen bestaan uit interferon- $\beta$, glatirameer acetaat, teriflunomide en dimethyl fumaraat (DMF). Hiervan zijn de laatste twee de eerste orale eerstelijnstherapieën die ontwikkeld werden voor de behandeling van MS. Wanneer de eerstelijnstherapieën onvoldoende effectief zijn, of in geval van agressief beginnend MS wordt overgegaan naar tweede- of derdelijnsbehandelingen, zoals alemtuzumab, natalizumab, fingolimod, ocrelizumab en cladribine. In het algemeen zijn deze tweede- en derdelijnsbehandelingen meer effectief, maar gaan deze gepaard met een complexer risico op bijwerkingen en vergen deze behandelingen intensievere monitoring.

\subsection{Wat is het doel van de studie?}

De laatste jaren zijn een aantal nieuwe immunomodulerende therapieën voor de behandeling van MS op de markt gekomen, zonder dat het werkingsmechanisme volledig duidelijk was. Aangezien patiënten en hun dokters tussen verschillende behandelingsopties kunnen en moeten kiezen, is het belangrijk om te weten hoe 
deze therapieën precies werken. Op deze manier kunnen voorspellingen gedaan worden over de effectiviteit en veiligheid van een therapie voor een specifieke patiënt en kan eveneens extra aandacht worden besteed aan typische (bij)werkingen van de therapie die verwacht kunnen worden als gevolg van het mechanisme. In 2013 kwam DMF op de markt als eerstelijnstherapie. DMF werd initieel gebruikt voor de behandeling van psoriasis, maar werd eveneens veilig en efficiënt bevonden voor de behandeling van MS. Desondanks was het werkingsmechanisme van DMF op dat moment grotendeels onbekend. Het doel van deze studie is om te achterhalen op welke manier DMF kan bijdragen tot het herstellen van de verstoorde immuun balans.

Verder is er in deze studie opgemerkt dat een specifiek subtype van B-cellen, namelijk de DN B-cellen, door DMF wordt gereduceerd. Voorgaand onderzoek van onze onderzoeksgroep had aangetoond dat er een toename is van dit B-cel subtype in het bloed van MS-patiënten en dat deze cellen mogelijk een pathogene rol hebben in MS aangezien ze teruggevonden zijn in de cerebrospinale vloeistof van MS-patiënten en een pro-inflammatoir cytokine profiel vertoonden. Daarom was het voor onze onderzoeksgroep belangrijk om te bekijken welk ontwikkelingsfenotype(s) en migratiecapaciteit deze cellen vertonen.

\subsection{Wat zijn de resultaten van de studie?}

\section{Hoe verandert DMF behandeling de immuunbalans van MS-patiënten?}

DMF is een fumaraat ester, een klein molecule met immunomodulerende, antiinflammatoire en anti-oxidatieve eigenschappen. In MS-patiënten was reeds geweten dat DMF effectief en veilig is. Echter was het effect van DMF op het aangeboren en verworven immuunsysteem van MS-patiënten nog niet bestudeerd. DMF wordt in de darm grotendeels omgezet in monomethyl fumaraat (MMF), hetgeen (mede-)verantwoordelijk is voor de verschillende werkingsmechanismen van DMF.

In Hoofdstuk 2 werden de longitudinale effecten van DMF behandeling bestudeerd in een follow-up studie met 21 RRMS-patiënten. Hier werd eerst gekeken naar klinische parameters zoals medische beeldvorming, ziektescore (expanded disability status score, EDSS), motor en cognitieve functies en vermoeidheid. DMF werd in onze studie goed getolereerd; slechts 2 MS-patiënten 
hebben de studie verlaten wegens langdurige bijwerkingen zoals gastrointestinale symptomen en opvliegers (flushing). We zagen een verbetering in cognitieve functie, terwijl motorische functies en de vermoeidheid van de patiënten stabiel bleven gedurende de behandelingsperiode. Daarnaast waren van 18 MS-patiënten MRI beelden beschikbaar. Hierbij werd aangetoond dat 4/18 MSpatiënten een reductie vertoonden in het volume van de laesies of een algemene verbetering ten opzichte van MRI beelden genomen voor de DMF-behandeling. De overige 14 MS-patiënten vertoonden een stabiel ziektebeeld zonder nieuwe of vergrote laesies.

Vervolgens werd het totale aantal van immuuncel subtypes bepaalde in het perifere bloed vóór en op verschillende tijdstippen na behandeling met DMF. Hierbij werd aangetoond dat vooral de cellen van het verworven immuunsysteem afnamen na 12 maanden behandeling. Het totaal aantal pro-inflammatoire immuuncel subtypes, zoals geheugen $\mathrm{CD}^{+}$en $\mathrm{CD}^{+} \mathrm{T}$-cellen, geheugen $\mathrm{B}$-cellen en DN B-cellen, was verlaagd na 12 maanden behandeling. Naïeve CD4 ${ }^{+}$en $C D 8^{+}$ T-cellen, naïeve en transitionele B-cellen, die allen niet betrokken zijn bij de pathologie van MS, bleven onveranderd na 12 maanden behandeling. Ook het totaal aantal monocyten en neutrofielen, bleef stabiel na 12 maanden behandeling. Zelfs na twaalf maanden bleef dit effect voortduren. Verder werd een vermindering waargenomen in het totale aantal pro-inflammatoire NK cellen, terwijl het aantal regulatoire NK-cellen constant bleef.

Ook de relatieve frequenties van de immuuncel subtypes werden gemeten in het perifere bloed om op deze manier de relatieve samenstelling van het overblijvende lymfocyt repertoire na DMF behandeling te evalueren. Na behandeling met DMF waren de frequenties van verschillende subtypes van geheugen $\mathrm{CD} 4^{+}$en $\mathrm{CD} 8^{+} \mathrm{T}$ cellen verlaagd. De frequenties van naïeve $C D 4^{+}$en $C D 8^{+} \mathrm{T}$-cellen waren dan weer verhoogd in MS-patiënten behandeld met DMF. DMF behandeling vertoonde vergelijkbare effecten op $B$-cellen, met een vermindering van de frequenties van geheugen B-cellen en DN B-cellen en een verhoging van naïeve en transitionele $B$-cellen in behandelde MS-patiënten. Dit leidde tot de conclusie dat de depletie van geheugen $\mathrm{T}$ - en B-cellen één van de werkingsmechanismen is van DMF behandeling in MS. Op deze manier heeft DMF de mogelijkheid om de verstoorde 
balans van het immuunsysteem te herstellen tot een minder pro-inflammatoir profiel.

Ex vivo stimulatie van $\mathrm{CD}^{+}{ }^{+}$T-cellen van MS-patiënten behandeld met DMF resulteerde in een verlaagde frequentie van pro-inflammatoire granulocytemacrophage colony-stimulating factor, IFN- $\gamma^{+}$, en $\mathrm{IL}_{-1} 17^{+} \mathrm{CD} 4^{+}$T-cellen. De frequentie van $\mathrm{IL}_{-} 4^{+}$en $\mathrm{IL}-10^{+} \mathrm{CD} 4^{+}$T-cellen bleef constant. Deze resultaten suggereerden dat DMF behandeling de inflammatoire Th1 en Th17 respons veranderde naar een meer neutrale immuunrespons in MS-patiënten.

Als conclusie bleek dat DMF vooral de pro-inflammatoire immuuncellen deed afnemen waardoor de verstoorde balans in het immuunsysteem hersteld werd.

\section{Worden B-cellen direct beïnvloed door DMF behandeling?}

We onderzochten het effect van DMF behandeling op verschillende B-cel functies. In Hoofdstuk 2 toonden we aan dat DMF behandeling het totale aantal B-cellen vermindert en dat het de verdeling van verschillende B-cel subtypes verandert, met onder andere een vermindering van de frequentie aan geheugen B-cel subtypes. In Hoofdstuk 3 werd DMF in vitro toegevoegd aan B-cellen van gezonde controles of onbehandelde MS-patiënten waarna het effect hiervan op verschillende B-cel functies werd bepaald. Zo werd gekeken naar productie van cytokines en expressie van markers voor overleving, antigeen presentatie en costimulatie.

Vanwege het verminderde totale aantal B-cellen in DMF behandelde MS-patiënten, onderzochten we eerst of DMF apoptose van B-cellen induceert. In vitro behandeling met DMF, maar niet met MMF, induceerde apoptose bij geactiveerde B-cellen van gezonde controles en MS-patiënten. Deze apoptose nam toe met een steeds hogere concentratie van DMF, met een sterker effect op B-cellen van MSpatiënten.

B-cellen zijn onder andere betrokken bij de pathologie van MS door de stimulatie van pro-inflammatoire T-cel responsen. Het zijn efficiënte antigeen presenterende cellen die ook costimulatoire moleculen tot expressie brengen voor T-cel activatie. Zoals beschreven in Hoofdstuk 3, was de expressie van moleculen betrokken bij deze processen op B-cellen verlaagd na in vitro behandeling met DMF. 
In de follow-up studie, beschreven in Hoofdstuk 2 was de frequentie van transitionele B-cellen in het perifere bloed van MS-patiënten verhoogd na behandeling met DMF. Van transitionele B-cellen is geweten dat ze kunnen differentiëren naar regulatoire B-cellen (Breg), die kunnen zorgen voor een onderdrukking van de immuunrespons. In Hoofdstuk 3 werd aangetoond dat directe behandeling van B-cellen met DMF resulteert in een trend tot een verhoogde Breg frequentie. Bovendien was de frequentie van pro-inflammatoire tumor necrose factor- $\mathrm{a}^{+} \mathrm{B}$-cellen verlaagd na behandeling met MMF. Deze data dragen bij tot de door DMF geïnduceerde shift in immuunbalans naar een antiinflammatoir profiel.

\section{Zijn DN B-cellen belangrijk voor de pathogenese van MS?}

In Hoofdstuk 2 werd een verlaagd percentage geheugen en DN B-cellen aangetoond in het perifere bloed van MS-patiënten behandeld met DMF. DN Bcellen zijn geassocieerd met immuunveroudering. In de geriatrische populatie is er aangetoond dat DN B-cellen in aantal vermeerderd zijn door een proces dat inflamm-aging genoemd wordt, hetgeen duidt op frequente ontstekingsreacties als gevolg van ouderdom. Op dit moment is nog niet veel geweten over DN Bcellen, en zeker niet over hun (mogelijke) rol in MS. Onze onderzoeksgroep heeft eerder al aangetoond dat er een verhoogde frequentie is van DN B-cellen in het bloed van jonge MS-patiënten. Deze DN B-cellen werden ook teruggevonden in de cerebrospinale vloeistof van MS-patiënten en vertoonden een pro-inflammatoir cytokine profiel. DN B-cellen spelen dus mogelijk een rol in de pathologie van MS. In Hoofdstuk 4 werd bestudeerd in welke ontwikkelingsfase de DN B-cellen zich bevonden. Verder werd hun capaciteit tot migratie naar inflammatoir weefsel onderzocht.

DN B-cellen vertoonden een expressie profiel van ontwikkelingsmarkers dat sterk gelijkend was op dat van geheugen B-cellen. Bovendien waren de meerderheid van de DN B-cellen mature cellen en brachten ze immunoglobuline (Ig)G of IgA tot expressie. Deze Igs zijn betrokken bij de secundaire immuunrespons, wat opnieuw wijst op een geheugen fenotype. Er werden echter ook verschillen waargenomen tussen de DN en geheugen B-cellen. Zo was de frequentie van geactiveerde $\mathrm{CD}^{+} 5^{+}$cellen en $\mathrm{IgA}^{+}$cellen lager bij de DN B-cellen. Over het algemeen kan besloten worden dat de DN B-cellen zich in een ontwikkelingsfase 
bevinden tussen de naïeve en geheugen B-cellen in. Verder werden geen verschillen gevonden in de onderzochte markers bij DN B-cellen van gezonde controles en MS-patiënten, wat erop wijst dat DN B-cellen zich in dezelfde ontwikkelingsfase bevinden bij beide groepen.

Verder hebben we onderzocht of CD21 low B-cellen (een ander B-cel subtype gerelateerd aan veroudering) en DN B-cellen van MS patiënten T-bet tot expressie brengen. T-bet is een transcriptiefactor die reeds beschreven werd in immuunverouderde B-cellen in muizen met systemische lupus erythematosus. Daar werd bovendien aangetoond dat de T-bet ${ }^{+}$B-cellen verantwoordelijk zijn voor de productie van auto-antilichamen. Bij MS-patiënten waren ongeveer $23 \%$ van de DN B-cellen positief voor T-bet, in vergelijking met ongeveer $70 \%$ van de CD21 low B-cellen (Hoofdstuk 4). Dit leert ons dat CD21 low B-cellen waarschijnlijk het humane equivalent zijn van de T-bet $^{+} \mathrm{B}$-cellen die beschreven werden in muizen. Voorts suggereren deze resultaten dat er verschillende subtypes bestaan in de DN B-cel populatie die elk een andere functie kunnen uitoefenen in de pathologie van MS.

Om de verhoogde frequentie van DN B-cellen in de cerebrospinale vloeistof van MS-patiënten verder te verklaren, werd de migratie capaciteit (fenotype en functie) en expressie van adhesiemoleculen op DN B-cellen onderzocht in Hoofdstuk 4. De gemeten pro-inflammatoire chemokine receptoren en adhesiemoleculen kwamen tot expressie op de DN B-cellen. Verder werd aangetoond dat DN B-cellen van MS-patiënten de capaciteit hebben om te migreren naar de pro-inflammatoire chemokines CXCL13 en CXCL10 met een efficiëntie die vergelijkbaar was met die van geheugen B-cellen. Ons onderzoek bewees dus dat DN B-cellen in staat zijn om te migreren naar pro-inflammatoire chemokines die aanwezig zijn in het centrale zenuwstelsel. Een volgende stap zou zijn om de aanwezigheid van DN B-cellen aan te tonen in hersenlaesies van MSpatiënten.

Verder hebben we in Hoofdstuk 4 het proliferatie en activatie potentieel van DN $\mathrm{B}$-cellen onderzocht. $\mathrm{Na}$ in vitro stimulatie vertoonden de DN B-cellen van gezonde controles de sterkste proliferatie en hoogste expressie van activatie markers (CD80 en CD25) in vergelijking met naïeve en geheugen B-cellen. Echter, door het lage aantal geïncludeerde gezonde controles was dit resultaat niet significant. 
Deze resultaten spreken een vroegere theorie tegen dat DN B-cellen functioneel uitgeput zouden zijn. Om de rol van de DN B-cellen in de pathologie van MS verder te onderzoeken, zou een volgende stap zijn om te bekijken of MS-patiënten met een toename in de DN B-cellen ook een ernstiger klinisch verloop van de ziekte hebben.

\subsection{Wat is de conclusie van de studies?}

De resultaten die in dit doctoraat behaald werden, bewijzen dat het werkingsmechanisme van DMF bij MS-patiënten multifactorieel is. In het algemeen oefent DMF een immuunmodulerend effect uit en verschuift het de verstoorde immuunbalans van MS-patiënten weg van een pro-inflammatoir profiel. Verder reduceert DMF selectief CD8 ${ }^{+} \mathrm{T}$-cellen en inflammatoire geheugen subtypes van T- en B-cellen in MS-patiënten. DMF induceert apoptose van B-cellen en reduceert de expressie van markers voor overleving, antigeen presentatie en costimulatie op B-cellen. Bijgevolg kan DMF behandeling de auto-immuunreacties in de pathogenese van MS verminderen door het aantal en de functie van proinflammatoire immuuncellen te verminderen via moleculaire mechanismen.

DMF behandeling vermindert de frequentie van DN B-cellen in het perifere bloed van MS-patiënten. Deze immuunverouderde DN B-cellen zijn mature cellen die een geheugen fenotype hebben maar toch in een vroegere ontwikkelingsfase lijken te zitten ten opzichte van de geheugen B-cellen. Verschillende subtypes van DN B-cellen zullen mogelijk verschillende functies uitvoeren. Er moet nog bepaald worden welk subtype of welke subtypes betrokken zijn in de pathologie van MS.

Verder kunnen DN B-cellen na in vitro stimulatie prolifereren en geactiveerd worden. Ook hebben ze een hoge capaciteit om te migreren naar proinflammatoire chemokines die betrokken zijn bij B-cel migratie naar het centrale zenuwstelsel van MS-patiënten. Al deze bevindingen samen wijzen naar de betrokkenheid van DN B-cellen in de pathogenese van MS. Verder onderzoek is nodig om de onderliggende moleculaire mechanismen van de verhoogde DN B-cel frequenties bij MS te identificeren. Bovendien moeten de verschillende functies van DN B-cellen in de pathologie van MS verder onderzocht worden. 


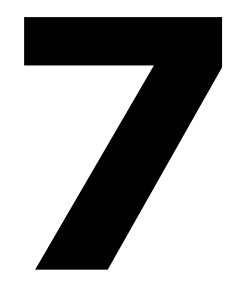

Valorisation addendum 


\section{Valorisation addendum}

In the Western world, multiple sclerosis (MS) is the most common neuroinflammatory disorder of the central nervous system (CNS) in young adults aged 20 - 40 years. MS is the main cause of neurological impairment and disability amongst young adults, affecting more than 2 million people worldwide (1). The prevalence and incidence rates of MS have been gradually increasing worldwide over the past two decades (2). The prevalence of MS in Belgium is approximately 118 individuals per 100,000 inhabitants and in the Netherlands it is estimated to be 88 per 100,000 inhabitants $(392,393)$. MS patients have a wide range of symptoms, from numbness in limbs and paralysis to fatigue and cognitive symptoms $(1,24)$. Furthermore, these symptoms cause MS patients to be vulnerable to depression since the quality of life is decreased through physical, social and psychological losses or problems. In terms of employment in Belgium, a high rate of $56 \%$ of MS patients below the retirement age are unemployed (394). For the MS patients who have the possibility of being employed, productivity at work is affected in $85 \%$ of the patients. This is mostly due to fatigue and cognitive difficulties, reflecting a high economic burden on society as a whole, and a financial burden on the patients themselves (394). Mean annual costs for an MS patient with an Expanded Disability Status Scale (EDSS) between 0 and 3 (mild MS) is $€ 26,400$ and is more than double for patients with an EDSS between 7 and 9 (severe MS, progression). In addition, the mean cost of a relapse was estimated to be $€ 3,000$ (394). Since MS adversely affects the quality of life and is accompanied by a high socio-economic burden, it is important to study the disease mechanisms of MS in more detail. In addition, treating MS patients as early as possible with an effective treatment is crucial in order to delay disease progression.

Until now, there still is no cure available for MS. However, over the past two decades, the treatment landscape has changed tremendously and several novel disease modifying therapies (DMTs) have been added to the armamentarium of MS treatment. At this moment, 12 treatments have been approved for MS, which present 10 different substance classes with different mechanisms of action (181). Although some of these treatments are target specific, multiple MS treatments have no clearly defined mechanism of action or a multifactorial and not fully 
unraveled mechanism at the moment of commercial release (181). In order to maximize patient benefit, appropriate selection of MS therapies is crucial. For this reason it is important to have insight into the working mechanism of these treatments. Although much has already been learnt about the pathogenesis of MS by the administration of treatment, unravelling the unknown mechanisms of action additionally leads to improved insight into and more specific knowledge about the role of the different immune cell subtypes in MS pathology.

The research that I performed for this thesis revealed different modes of action of dimethyl fumarate (DMF) therapy that are responsible for the immunomodulatory and positive clinical effects that have been seen in MS patients.

\section{The importance of investigating the working mechanism of DMF therapy in MS patients}

At the start of this study, the therapeutic mechanism of action of DMF in MS patients was largely unclear. Our research led to a better understanding of the different immune cell subtypes targeted by DMF in MS patients. For instance, our research indicated that DMF redirects the disrupted immune balance of MS patients away from a pro-inflammatory immune response by specifically decreasing memory and effector memory $T$ and $B$ cells. The same immune cell subtypes were shown to be targeted by DMF in different study populations originating from different countries (Spain, Germany, USA and Belgium) (199, $304,308,309,316)$. This indicates that the effects of DMF on the immune system are reproducible, even though it has multiple working mechanisms. Additionally, the fact that similar results are observed in different geographical regions suggests that the therapy is reliable and that its effects are vast. The effect of DMF on specific immune cell subtypes can be used to build upon with additional research, to enable to make predictions in the future which specific MS patient/sub group would benefit from this therapy. By enabling physicians to make these informed decisions based on the specific patients' immune cell profile, these patients would be treated sooner with a more suitable treatment. When patients would benefit from an effective treatment earlier in their disease, the disease would remain stable for a longer period of time, which results in reduced chances to develop severe disabilities. Additionally, a patient with a more stable disease course would be able to fully participate in society for a longer period of time, 
which has positive effects on the ability to remain employed, the quality of life, financial state, physical and emotional wellbeing, the healthcare system, stress on the family, friends and caregivers of the patient, and society as a whole.

In this study, side effects that were observed due to the use of DMF were mainly flushing and gastro-intestinal symptoms. This caused two patients to drop out of the study. Therefore, careful clinical evaluation of severe flushing and gastrointestinal symptoms should be considered. Furthermore, one MS patient dropped out due to lymphopenia. DMF causes pronounced lymphopenia in $4-6 \%$ of MS patients (361). Thus, several studies, including our study, have demonstrated that when administering DMF to MS patients it is important to monitor the absolute lymphocyte number, as this will reduce the risk of developing prolonged lymphopenia and will secure the safety of the patient. The relatively low incidence and severity of adverse events, as well as the high efficacy of the drug make DMF therapy a popular treatment option, which can be used to treat the majority of relapsing remitting MS (RRMS) patients in the first-line setting. Additionally, in most patients, some side effects that do occur will decrease in severity or will even completely disappear after a period of time. Because of this, a long adherence to DMF treatment is possible, with positive effects on the disease course. The longer a patient can benefit from first-line treatment, the longer a treatment switch to second- or third line can be postponed, potentially protecting the patient from more severe adverse events down the line. In addition, since DMF is an oral treatment, the drug can be prescribed by a specialist, but also by a general practitioner (for repeated doses). The drug can be taken orally by the patients at home, limiting the hospital visits and increasing the comfort for the MS patients.

Another important finding is that DMF is fully effective on immune cells after 6 months of treatment. The absolute lymphocyte number plateaued after 12 months of DMF treatment (361). These results indicate that after 6 months of treatment, physicians can cautiously assess the effectiveness of the treatment on immune cells, treatment adherence and side-effect profile. A next step would be to further investigate the most critical immune cell subtypes affected by DMF in this study. Continued monitoring of these subtypes in a large cohort may then improve our ability to identify poor responders and improve treatment outcomes. 
In this thesis I showed that pro-inflammatory cytokine production by $\mathrm{T}$ cells and $B$ cells is reduced in DMF-treated MS patients, shifting the Th1/Th17 and proinflammatory B cell response to an anti-inflammatory response. Functionally, DMF treatment induces apoptosis of B cells and reduces the expression of survival, antigen presentation and costimulatory markers on $B$ cells. It may be possible to extrapolate these uncovered working mechanisms of DMF to other auto-immune diseases where DMF could possibly be used as a treatment. Furthermore, the uncovered mechanism of action of DMF and its ability to shift the immune profile can facilitate the development of next generation therapies for MS. In addition, the discovery of specific immune cell subtypes that are targeted by DMF could lead to tailored therapy. Finally, if in the future it is desired to combine several MS therapies with DMF for a better treatment efficacy, the acquisition of sufficient knowledge about the mechanism of action of DMF as performed in this study would ensure patient safety. Finally, understanding the mechanism of action of DMF on the immune system of MS patients gave us more insight in the role of these immune cells in the pathogenesis of MS.

\section{The importance of DN B cells in the pathology of MS}

Monoclonal antibodies that target CD20 expressing B cells have been shown to be a highly effective treatment option for MS patients (395). However, depleting the total $B$ cell population can result in lowered resistance against pathogens and higher rates of cancer and infection (395). Furthermore, therapies such as rituximab that target all CD20-positive B cells have been shown to also kill nonpathogenic $B$ cells. For these reasons it is important to identify disease-relevant $B$ cell subtypes among the vastly diverse peripheral B cell compartment to find alternative targeted therapies. DN B cells may be one of the B cell subtypes with potential as therapeutic target. Our research group previously showed that DN $B$ cells are abnormally elevated in the peripheral blood and cerebrospinal fluid (CSF) of MS patients. Moreover, DN B cells showed pro-inflammatory functional characteristics indicating that they might play a role in the pathogenesis of MS (68). In addition, we observed that DMF treatment decreased the frequency of DN $B$ cells in MS patients. In this thesis, the developmental phenotype and migratory capacity of DN B cells from MS patients were investigated in more detail. We showed that DN B cells have a mature and memory phenotype, though with earlier maturation features compared to class-switched memory (CSM) B cells. 
Furthermore, we have shown that DN B cells can become activated and that they have a high migratory capacity towards pro-inflammatory chemokines involved in B cell trafficking to the CNS in MS. Unraveling the phenotype of DN B cells is important in order to understand their functional relevance and possible involvement in MS pathogenesis. The finding of a high migratory capacity of DN $B$ cells towards pro-inflammatory cytokines provided more evidence that DN B cells could possibly contribute to the pathogenesis of MS, making them even more desirable to be targeted by biologics. The knowledge obtained from our studies could contribute to future targeting or modulating DN B cells for MS treatment. Furthermore, by next-generation sequencing of the $B$ cell receptor immunoglobulin genes (383), our research group showed that DN B cells of MS patients and healthy individuals share a common developmental pathway. Thus, information gathered on DN B cells of MS patients could also be implemented in the development of therapies for immune aging which could also contribute to the improvement of general healthcare.

When specifically targeting pathologically relevant DN B cells, the risk of bacterial and viral infections and neoplasms would be diminished compared to targeting the entire $B$ cell population. This means that less side effects are expected when tailoring such a more selective B cell therapy. Furthermore, various other B cell subtypes produce anti-inflammatory cytokines, induce Treg and/or inhibit effector $T$ helper cells, which counteracts the inflammatory processes and thus is beneficial for the pathogenesis of MS. However, previous research performed by our research group and results in this thesis show that the effect of targeting DN $B$ cells would not only be contained to DN B cells only, as this B cell subtype could also have an indirect effect on T cells. DN B cell depleting therapy could eventually be used as a personalized treatment for patients with an increased frequency of DN B cells. However, more research is needed in order to determine whether antiDN B cell therapy has a significant effect on the pathogenesis of MS by itself. When anti-DN B cell therapy alone is not sufficient, the therapy could also be used as an add-on treatment.

However, in order to tailor a therapy for depleting DN B cells in MS patients further research is needed to find out which markers or targets are specifically expressed by DN B cells and are absent in other B cell subtypes. This could be performed by 
RNA sequencing. Furthermore, it would be interesting to investigate DN B cellassociated signaling pathways that may assist us to better understand the association between the elevations of DN B cells in aging and in autoimmune disease. A possible scenario in the search for a marker or target would be to consider the pathways related to DN B cell cytokine production, migratory pathway, activation pathway, IL-21/granzyme B pathway, T-bet or Toll-like Receptor pathways. If one of aforementioned pathways is relevant and can be targeted for treatment, the first step would be to review drugs that are already on the market or in development, for instance for the treatment of other diseases, that have an effect on proteins that are involved in previously discussed pathways. Another approach would be to target the involved proteins with monoclonal antibodies that may or may not already be developed. At this moment several drugs that block the above mentioned pathways are in development. For instance Human r-IL-21 is available for clinical use to target tumors $(396,397)$. Several TLR7 or 9 antagonists are tested in clinical phase II trials in order to treat diseases like psoriasis, optic neuritis, diffuse large B cell lymphoma and Sjögren's syndrome (398). In conclusion, although in our research some characteristics of DN B cells were unraveled, it is clear that more research is needed to eventually target DN B cells for therapeutic purposes. 



\section{Reference list}

1. Compston A, Coles A. Multiple sclerosis. Lancet (London, England). 2008;372(9648):1502-17.

2. Browne P, Chandraratna D, Angood C, Tremlett H, Baker C, Taylor BV, et al. Atlas of Multiple Sclerosis 2013: A growing global problem with widespread inequity. Neurology. 2014;83(11):1022-4.

3. Dargahi N, Katsara M, Tselios T, Androutsou ME, de Courten M, Matsoukas J, et al. Multiple Sclerosis: Immunopathology and Treatment Update. Brain sciences. 2017;7(7).

4. Thompson AJ, Baranzini SE, Geurts J, Hemmer B, Ciccarelli O. Multiple sclerosis. Lancet (London, England). 2018;391(10130):1622-36.

5. Bostrom I, Landtblom AM. Does the changing sex ratio of multiple sclerosis give opportunities for intervention? Acta neurologica Scandinavica. 2015;132(199):42-5.

6. Orton SM, Herrera BM, Yee IM, Valdar W, Ramagopalan SV, Sadovnick $A D$, et al. Sex ratio of multiple sclerosis in Canada: a longitudinal study. The Lancet Neurology. 2006;5(11):932-6.

7. Doshi A, Chataway J. Multiple sclerosis, a treatable disease. Clinical medicine (London, England). 2016;16(Suppl 6):s53-s9.

8. Hafler DA. Multiple sclerosis. The Journal of clinical investigation. 2004;113(6):788-94.

9. Hawker K, Frohman E. Multiple sclerosis. Primary care. 2004;31(1):20126.

10. Weinshenker BG, Bass B, Rice GP, Noseworthy J, Carriere W, Baskerville J, et al. The natural history of multiple sclerosis: a geographically based study. I. Clinical course and disability. Brain : a journal of neurology. $1989 ; 112$ ( Pt 1):13346.

11. Lorscheider J, Buzzard K, Jokubaitis V, Spelman T, Havrdova E, Horakova $D$, et al. Defining secondary progressive multiple sclerosis. Brain : a journal of neurology. 2016;139(Pt 9):2395-405.

12. Høglund RA, Maghazachi AA. Multiple sclerosis and the role of immune cells. World J Exp Med. 2014;4(3):27-37.

13. Rizzo MA, Hadjimichael OC, Preiningerova J, Vollmer TL. Prevalence and treatment of spasticity reported by multiple sclerosis patients. Multiple sclerosis (Houndmills, Basingstoke, England). 2004;10(5):589-95.

14. Stuke K, Flachenecker P, Zettl UK, Elias WG, Freidel M, Haas J, et al. Symptomatology of MS: results from the German MS Registry. J Neurol. 2009;256(11):1932-5.

15. WHO. MS atlas. 2008.

16. Flachenecker $P$, Henze $T$, Zettl UK. Spasticity in patients with multiple sclerosis--clinical characteristics, treatment and quality of life. Acta neurologica Scandinavica. 2014;129(3):154-62.

17. Kister I, Bacon TE, Chamot E, Salter AR, Cutter GR, Kalina JT, et al. Natural history of multiple sclerosis symptoms. International journal of MS care. 2013;15(3):146-58.

18. Koch M, Mostert J, Heersema D, De Keyser J. Tremor in multiple sclerosis. J Neurol. 2007;254(2):133-45.

19. Lerdal A, Celius EG, Krupp L, Dahl AA. A prospective study of patterns of fatigue in multiple sclerosis. European journal of neurology. 2007;14(12):133843. 
20. Amato MP, Ponziani G, Siracusa G, Sorbi S. Cognitive dysfunction in earlyonset multiple sclerosis: a reappraisal after 10 years. Archives of neurology. 2001;58(10):1602-6.

21. Hviid LE, Healy BC, Rintell DJ, Chitnis T, Weiner HL, Glanz BI. Patient reported outcomes in benign multiple sclerosis. Multiple sclerosis (Houndmills, Basingstoke, England). 2011;17(7):876-84.

22. Amato MP, Zipoli V, Goretti B, Portaccio E, De Caro MF, Ricchiuti L, et al. Benign multiple sclerosis: cognitive, psychological and social aspects in a clinical cohort. J Neurol. 2006;253(8):1054-9.

23. Kobelt G, Thompson A, Berg J, Gannedahl M, Eriksson J. New insights into the burden and costs of multiple sclerosis in Europe. Multiple sclerosis (Houndmills, Basingstoke, England). 2017;23(8):1123-36.

24. Wakerley B NR, Malik O. Multiple sclerosis. Medicine. 2008(36(12)):6259.

25. Tremlett H, Yinshan Z, Devonshire V. Natural history of secondaryprogressive multiple sclerosis. Multiple sclerosis (Houndmills, Basingstoke, England). 2008;14(3):314-24.

26. Cree BA, Gourraud PA, Oksenberg JR, Bevan C, Crabtree-Hartman E, Gelfand JM, et al. Long-term evolution of multiple sclerosis disability in the treatment era. Annals of neurology. 2016;80(4):499-510.

27. Gitto L. Living with Multiple Sclerosis in Europe: Pharmacological Treatments, Cost of Illness, and Health-Related Quality of Life Across Countries. In: Zagon IS, McLaughlin PJ, editors. Multiple Sclerosis: Perspectives in Treatment and Pathogenesis. Brisbane (AU): Codon Publications

Copyright: The Authors.; 2017.

28. Freeman J, Gorst T, Gunn H, Robens S. "A non-person to the rest of the world": experiences of social isolation amongst severely impaired people with multiple sclerosis. Disability and rehabilitation. 2019:1-9.

29. Mikula P, Timkova V, Linkova M, Vitkova M, Szilasiova J, Nagyova I. Fatigue and Suicidal Ideation in People With Multiple Sclerosis: The Role of Social Support. Frontiers in psychology. 2020;11:504.

30. Ascherio A, Munger KL. Epidemiology of Multiple Sclerosis: From Risk Factors to Prevention-An Update. Seminars in neurology. 2016;36(2):103-14.

31. DeLorenze GN, Munger KL, Lennette ET, Orentreich N, Vogelman JH, Ascherio A. Epstein-Barr virus and multiple sclerosis: evidence of association from a prospective study with long-term follow-up. Archives of neurology. 2006;63(6):839-44.

32. Healy BC, Ali EN, Guttmann CR, Chitnis T, Glanz BI, Buckle G, et al. Smoking and disease progression in multiple sclerosis. Archives of neurology. 2009;66(7):858-64.

33. Kleinewietfeld M, Manzel A, Titze J, Kvakan $H$, Yosef N, Linker RA, et al. Sodium chloride drives autoimmune disease by the induction of pathogenic TH17 cells. Nature. 2013;496(7446):518-22.

34. Riccio P, Rossano R, Larocca M, Trotta V, Mennella I, Vitaglione $P$, et al. Anti-inflammatory nutritional intervention in patients with relapsing-remitting and primary-progressive multiple sclerosis: A pilot study. Experimental biology and medicine (Maywood, NJ). 2016;241(6):620-35.

35. Salzer J, Hallmans G, Nystrom M, Stenlund H, Wadell G, Sundstrom P. Vitamin $D$ as a protective factor in multiple sclerosis. Neurology. $2012 ; 79(21): 2140-5$. 
36. Simpson S, Jr., Blizzard L, Otahal P, Van der Mei I, Taylor B. Latitude is significantly associated with the prevalence of multiple sclerosis: a meta-analysis. Journal of neurology, neurosurgery, and psychiatry. 2011;82(10):1132-41.

37. Vanheusden $M$, Broux $B$, Welten SP, Peeters LM, Panagioti $E$, Van Wijmeersch $B$, et al. Cytomegalovirus infection exacerbates autoimmune mediated neuroinflammation. Scientific reports. 2017;7(1):663.

38. Pistono C, Osera C, Boiocchi C, Mallucci G, Cuccia M, Bergamaschi R, et al. What's new about oral treatments in Multiple Sclerosis? Immunogenetics still under question. Pharmacological research. 2017;120:279-93.

39. Sawcer S, Franklin RJ, Ban M. Multiple sclerosis genetics. The Lancet Neurology. 2014;13(7):700-9.

40. Sawcer S, Hellenthal G, Pirinen M, Spencer CC, Patsopoulos NA, Moutsianas L, et al. Genetic risk and a primary role for cell-mediated immune mechanisms in multiple sclerosis. Nature. 2011;476(7359):214-9.

41. Patsopoulos NA, Barcellos LF, Hintzen RQ, Schaefer C, van Duijn CM, Noble JA, et al. Fine-mapping the genetic association of the major histocompatibility complex in multiple sclerosis: HLA and non-HLA effects. PLoS genetics. 2013;9(11):e1003926.

42. Hafler DA, Compston A, Sawcer S, Lander ES, Daly MJ, De Jager PL, et al. Risk alleles for multiple sclerosis identified by a genomewide study. The New England journal of medicine. 2007;357(9):851-62.

43. Consortium. IMSG. A systems biology approach uncovers cell-specific gene regulatory effects of genetic associations in multiple sclerosis. Nature communications. 2019;10(1):2236.

44. Smets I, Fiddes B, Garcia-Perez JE, He D, Mallants K, Liao W, et al. Multiple sclerosis risk variants alter expression of co-stimulatory genes in B cells. Brain : a journal of neurology. 2018;141(3):786-96.

45. Consortium. IMSG. Multiple sclerosis genomic map implicates peripheral immune cells and microglia in susceptibility. Science (New York, NY). 2019;365(6460).

46. Davis RS. Fc receptor-like molecules. Annual review of immunology. 2007;25:525-60.

47. Gil-Varea E, Spataro N, Villar LM, Tejeda-Velarde A, Midaglia L, Matesanz $F$, et al. Targeted resequencing reveals rare variants enrichment in multiple sclerosis susceptibility genes. Human mutation. 2020.

48. Hunter SF. Overview and diagnosis of multiple sclerosis. The American journal of managed care. 2016;22(6 Suppl):s141-50.

49. Thompson AJ, Banwell BL, Barkhof F, Carroll WM, Coetzee T, Comi G, et al. Diagnosis of multiple sclerosis: 2017 revisions of the McDonald criteria. The Lancet Neurology. 2018;17(2):162-73.

50. Giffroy X, Maes N, Albert A, Maquet P, Crielaard JM, Dive D. Multimodal evoked potentials for functional quantification and prognosis in multiple sclerosis. BMC neurology. 2016;16:83.

51. Legroux L, Arbour N. Multiple Sclerosis and T Lymphocytes: An Entangled Story. Journal of neuroimmune pharmacology : the official journal of the Society on NeuroImmune Pharmacology. 2015;10(4):528-46.

52. Li R, Patterson KR, Bar-Or A. Reassessing B cell contributions in multiple sclerosis. Nature immunology. 2018;19(7):696-707.

53. Chastain EM, Miller SD. Molecular mimicry as an inducing trigger for CNS autoimmune demyelinating disease. Immunological reviews. 2012;245(1):22738. 
54. Fujinami RS, von Herrath MG, Christen U, Whitton JL. Molecular mimicry, bystander activation, or viral persistence: infections and autoimmune disease. Clinical microbiology reviews. 2006;19(1):80-94.

55. Hemmer B, Kerschensteiner $M$, Korn T. Role of the innate and adaptive immune responses in the course of multiple sclerosis. The Lancet Neurology. 2015;14(4):406-19.

56. Bouwer HG, Hinrichs DJ. Adoptive transfer of experimental allergic encephalomyelitis: recipient response to myelin basic protein-reactive lymphocytes. Journal of neuroimmunology. 1994;54(1-2):87-98.

57. Dendrou CA, Fugger L, Friese MA. Immunopathology of multiple sclerosis. Nature reviews Immunology. 2015;15(9):545-58.

58. Prat $\mathrm{E}$, Martin R. The immunopathogenesis of multiple sclerosis. Journal of rehabilitation research and development. 2002;39(2):187-99.

59. Hauser SL, Waubant E, Arnold DL, Vollmer T, Antel J, Fox RJ, et al. B-cell depletion with rituximab in relapsing-remitting multiple sclerosis. The New England journal of medicine. 2008;358(7):676-88.

60. Magliozzi R, Howell O, Vora A, Serafini B, Nicholas R, Puopolo M, et al. Meningeal $\mathrm{B}$-cell follicles in secondary progressive multiple sclerosis associate with early onset of disease and severe cortical pathology. Brain : a journal of neurology. 2007;130(Pt 4):1089-104.

61. Owens GP, Bennett JL, Lassmann H, O'Connor KC, Ritchie AM, Shearer A, et al. Antibodies produced by clonally expanded plasma cells in multiple sclerosis cerebrospinal fluid. Annals of neurology. 2009;65(6):639-49.

62. Li R, Patterson KR, Bar-Or A. Reassessing B cell contributions in multiple sclerosis. Nature immunology. 2018.

63. Dittel BN. CD4 T cells: Balancing the coming and going of autoimmunemediated inflammation in the CNS. Brain, behavior, and immunity. 2008;22(4):421-30.

64. Korn T, Bettelli E, Oukka M, Kuchroo VK. IL-17 and Th17 Cells. Annual review of immunology. 2009;27:485-517.

65. Noster R, Riedel R, Mashreghi MF, Radbruch H, Harms L, Haftmann C, et al. IL-17 and GM-CSF expression are antagonistically regulated by human $T$ helper cells. Science translational medicine. 2014;6(241):241ra80.

66. Sallusto F, Lenig D, Forster R, Lipp M, Lanzavecchia A. Two subsets of memory $\mathrm{T}$ lymphocytes with distinct homing potentials and effector functions. Nature. 1999;401(6754):708-12.

67. Genain CP, Cannella B, Hauser SL, Raine CS. Identification of autoantibodies associated with myelin damage in multiple sclerosis. Nature medicine. 1999;5(2):170-5.

68. Claes N, Fraussen J, Vanheusden $M$, Hellings N, Stinissen $P$, Van Wijmeersch B, et al. Age-Associated B Cells with Proinflammatory Characteristics Are Expanded in a Proportion of Multiple Sclerosis Patients. Journal of immunology (Baltimore, Md : 1950). 2016;197(12):4576-83.

69. Niino M, Hirotani M, Miyazaki Y, Sasaki H. Memory and naive B-cell subsets in patients with multiple sclerosis. Neuroscience letters. 2009;464(1):74-8.

70. Wei C, Anolik J, Cappione A, Zheng B, Pugh-Bernard A, Brooks J, et al. A new population of cells lacking expression of $\mathrm{CD} 27$ represents a notable component of the B cell memory compartment in systemic lupus erythematosus. Journal of immunology (Baltimore, Md : 1950). 2007;178(10):6624-33. 
71. Neumann H, Medana IM, Bauer J, Lassmann H. Cytotoxic T lymphocytes in autoimmune and degenerative CNS diseases. Trends in neurosciences. 2002;25(6):313-9.

72. Babbe $H$, Roers A, Waisman A, Lassmann H, Goebels N, Hohlfeld $R$, et al. Clonal expansions of CD8(+) T cells dominate the $\mathrm{T}$ cell infiltrate in active multiple sclerosis lesions as shown by micromanipulation and single cell polymerase chain reaction. The Journal of experimental medicine. 2000;192(3):393-404.

73. Hauser SL, Bhan AK, Gilles F, Kemp M, Kerr C, Weiner HL. Immunohistochemical analysis of the cellular infiltrate in multiple sclerosis lesions. Annals of neurology. $1986 ; 19(6): 578-87$.

74. Salou M, Garcia A, Michel L, Gainche-Salmon A, Loussouarn D, Nicol B, et al. Expanded CD8 T-cell sharing between periphery and CNS in multiple sclerosis. Annals of clinical and translational neurology. 2015;2(6):609-22.

75. Kasper LH, Haque, A. \& Haque, S. Regulatory mechanims of the immune system in multiple sclerosis. T regulatory cells: turned on to turn off. J Neurol. 2007(254(Suppl 1)):I10.

76. Viglietta V, Baecher-Allan C, Weiner HL, Hafler DA. Loss of functional suppression by CD4+CD25+ regulatory $T$ cells in patients with multiple sclerosis. The Journal of experimental medicine. 2004;199(7):971-9.

77. Knippenberg S, Peelen E, Smolders J, Thewissen M, Menheere P, Cohen Tervaert JW, et al. Reduction in IL-10 producing B cells (Breg) in multiple sclerosis is accompanied by a reduced naive/memory Breg ratio during a relapse but not in remission. Journal of neuroimmunology. 2011;239(1-2):80-6.

78. Cooper MA, Fehniger TA, Turner SC, Chen KS, Ghaheri BA, Ghayur T, et al. Human natural killer cells: a unique innate immunoregulatory role for the CD56(bright) subset. Blood. 2001;97(10):3146-51.

79. Kastrukoff LF, Lau A, Wee R, Zecchini D, White R, Paty DW. Clinical relapses of multiple sclerosis are associated with 'novel' valleys in natural killer cell functional activity. Journal of neuroimmunology. 2003;145(1-2):103-14.

80. Laroni A, Armentani E, Kerlero de Rosbo N, Ivaldi F, Marcenaro E, Sivori $\mathrm{S}$, et al. Dysregulation of regulatory CD56(bright) NK cells/T cells interactions in multiple sclerosis. Journal of autoimmunity. 2016;72:8-18.

81. Morandi F, Horenstein AL, Chillemi A, Quarona V, Chiesa S, Imperatori A, et al. CD56brightCD16- NK Cells Produce Adenosine through a CD38-Mediated Pathway and Act as Regulatory Cells Inhibiting Autologous CD4+ $T$ Cell Proliferation. Journal of immunology (Baltimore, Md : 1950). 2015;195(3):96572.

82. Moretta A, Bottino C, Vitale M, Pende D, Cantoni C, Mingari MC, et al. Activating receptors and coreceptors involved in human natural killer cellmediated cytolysis. Annual review of immunology. 2001;19:197-223.

83. Ancuta P, Liu KY, Misra V, Wacleche VS, Gosselin A, Zhou X, et al. Transcriptional profiling reveals developmental relationship and distinct biological functions of CD16+ and CD16- monocyte subsets. BMC genomics. 2009;10:403. 84. Geissmann F, Jung S, Littman DR. Blood monocytes consist of two principal subsets with distinct migratory properties. Immunity. 2003;19(1):7182.

85. Waschbisch A, Schroder S, Schraudner D, Sammet L, Weksler B, Melms $A$, et al. Pivotal Role for CD16+ Monocytes in Immune Surveillance of the Central Nervous System. Journal of immunology (Baltimore, Md : 1950). 2016;196(4):1558-67. 
86. Claes N, Fraussen J, Stinissen P, Hupperts R, Somers V. B Cells Are Multifunctional Players in Multiple Sclerosis Pathogenesis: Insights from Therapeutic Interventions. Frontiers in immunology. 2015;6:642.

87. Pieper K, Grimbacher B, Eibel H. B-cell biology and development. The Journal of allergy and clinical immunology. 2013;131(4):959-71.

88. Colonna-Romano G, Bulati M, Aquino A, Pellicanò M, Vitello S, Lio D, et al. A double-negative (IgD-CD27-) B cell population is increased in the peripheral blood of elderly people. Mech Ageing Dev. 2009;130(10):681-90.

89. Wu YC, Kipling D, Dunn-Walters DK. The relationship between CD27 negative and positive $\mathrm{B}$ cell populations in human peripheral blood. Frontiers in immunology. 2011;2:81.

90. Colonna-Romano G, Bulati M, Aquino A, Pellicano M, Vitello S, Lio D, et al. A double-negative (IgD-CD27-) B cell population is increased in the peripheral blood of elderly people. Mechanisms of ageing and development. 2009;130(10):681-90.

91. Palanichamy A, Apeltsin L, Kuo TC, Sirota M, Wang S, Pitts SJ, et al. Immunoglobulin class-switched $B$ cells form an active immune axis between CNS and periphery in multiple sclerosis. Science translational medicine. 2014;6(248):248ra106.

92. von Budingen HC, Harrer MD, Kuenzle S, Meier M, Goebels N. Clonally expanded plasma cells in the cerebrospinal fluid of MS patients produce myelinspecific antibodies. European journal of immunology. 2008;38(7):2014-23.

93. Michel L, Chesneau M, Manceau P, Genty A, Garcia A, Salou M, et al. Unaltered regulatory $B$-cell frequency and function in patients with multiple sclerosis. Clinical immunology (Orlando, Fla). 2014;155(2):198-208.

94. Ray A, Mann MK, Basu S, Dittel BN. A case for regulatory B cells in controlling the severity of autoimmune-mediated inflammation in experimental autoimmune encephalomyelitis and multiple sclerosis. Journal of neuroimmunology. 2011;230(1-2):1-9.

95. Mauri $C$, Menon $M$. The expanding family of regulatory $B$ cells. International immunology. 2015;27(10):479-86.

96. Hauser SL, Waubant E, Arnold DL, Vollmer T, Antel J, Fox RJ, et al. B-cell depletion with rituximab in relapsing-remitting multiple sclerosis. The New England journal of medicine. 2008;358(7):676-88.

97. Hauser SL, Bar-Or A, Comi G, Giovannoni G, Hartung HP, Hemmer B, et al. Ocrelizumab versus Interferon Beta-1a in Relapsing Multiple Sclerosis. The New England journal of medicine. 2017;376(3):221-34.

98. Montalban X, Hauser SL, Kappos L, Arnold DL, Bar-Or A, Comi G, et al. Ocrelizumab versus Placebo in Primary Progressive Multiple Sclerosis. The New England journal of medicine. 2017;376(3):209-20.

99. Kappos L, Li D, Calabresi PA, O'Connor P, Bar-Or A, Barkhof F, et al. Ocrelizumab in relapsing-remitting multiple sclerosis: a phase 2, randomised, placebo-controlled, multicentre trial. Lancet (London, England). 2011;378(9805):1779-87.

100. Li R, Rezk A, Miyazaki Y, Hilgenberg E, Touil H, Shen P, et al. Proinflammatory GM-CSF-producing $B$ cells in multiple sclerosis and $B$ cell depletion therapy. Science translational medicine. 2015;7(310):310ra166.

101. Prineas JW, Graham JS. Multiple sclerosis: capping of surface immunoglobulin $G$ on macrophages engaged in myelin breakdown. Annals of neurology. $1981 ; 10(2): 149-58$. 
102. Lycke J. Monoclonal antibody therapies for the treatment of relapsingremitting multiple sclerosis: differentiating mechanisms and clinical outcomes. Therapeutic advances in neurological disorders. 2015;8(6):274-93.

103. Elliott C, Lindner M, Arthur A, Brennan K, Jarius S, Hussey J, et al. Functional identification of pathogenic autoantibody responses in patients with multiple sclerosis. Brain : a journal of neurology. 2012;135(Pt 6):1819-33.

104. Liu Y, Given KS, Harlow DE, Matschulat AM, Macklin WB, Bennett JL, et al. Myelin-specific multiple sclerosis antibodies cause complement-dependent oligodendrocyte loss and demyelination. Acta neuropathologica communications. 2017;5(1):25.

105. Piddlesden SJ, Lassmann H, Zimprich F, Morgan BP, Linington C. The demyelinating potential of antibodies to myelin oligodendrocyte glycoprotein is related to their ability to fix complement. The American journal of pathology. 1993;143(2):555-64.

106. Weber MS, Hemmer B, Cepok $S$. The role of antibodies in multiple sclerosis. Biochimica et biophysica acta. 2011;1812(2):239-45.

107. Villar LM, Masjuan J, Sadaba MC, Gonzalez-Porque P, Plaza J, Bootello A, et al. Early differential diagnosis of multiple sclerosis using a new oligoclonal band test. Archives of neurology. 2005;62(4):574-7.

108. Villar LM, Sadaba MC, Roldan E, Masjuan J, Gonzalez-Porque P, Villarrubia $\mathrm{N}$, et al. Intrathecal synthesis of oligoclonal IgM against myelin lipids predicts an aggressive disease course in MS. The Journal of clinical investigation. 2005;115(1):187-94.

109. Ayoglu B, Mitsios N, Kockum I, Khademi M, Zandian A, Sjoberg R, et al. Anoctamin 2 identified as an autoimmune target in multiple sclerosis. Proceedings of the National Academy of Sciences of the United States of America. 2016;113(8):2188-93.

110. de Bock L, Fraussen J, Villar LM, Alvarez-Cermeno JC, Van Wijmeersch B, van Pesch V, et al. Anti-SPAG16 antibodies in primary progressive multiple sclerosis are associated with an elevated progression index. European journal of neurology. 2016;23(4):722-8.

111. Fraussen J, Claes N, de Bock L, Somers V. Targets of the humoral autoimmune response in multiple sclerosis. Autoimmunity reviews. 2014;13(11):1126-37.

112. Sellebjerg F, Christiansen M, Garred P. MBP, anti-MBP and anti-PLP antibodies, and intrathecal complement activation in multiple sclerosis. Multiple sclerosis (Houndmills, Basingstoke, England). 1998;4(3):127-31.

113. Duddy M, Niino M, Adatia F, Hebert S, Freedman M, Atkins H, et al. Distinct effector cytokine profiles of memory and naive human $B$ cell subsets and implication in multiple sclerosis. Journal of immunology (Baltimore, Md : 1950). 2007;178(10):6092-9.

114. Li R, Rezk A, Healy LM, Muirhead G, Prat A, Gommerman JL, et al. Cytokine-Defined B Cell Responses as Therapeutic Targets in Multiple Sclerosis. Frontiers in immunology. 2015;6:626.

115. Bar-Or A, Fawaz L, Fan B, Darlington PJ, Rieger A, Ghorayeb C, et al. Abnormal B-cell cytokine responses a trigger of T-cell-mediated disease in MS? Annals of neurology. 2010;67(4):452-61.

116. Barr TA, Shen P, Brown S, Lampropoulou V, Roch T, Lawrie S, et al. B cell depletion therapy ameliorates autoimmune disease through ablation of IL-6producing B cells. The Journal of experimental medicine. 2012;209(5):1001-10. 
117. Miyazaki Y, Li R, Rezk A, Misirliyan H, Moore C, Farooqi N, et al. A novel microRNA-132-sirtuin-1 axis underlies aberrant $B$-cell cytokine regulation in patients with relapsing-remitting multiple sclerosis [corrected]. Plos one. 2014;9(8):e105421.

118. Pierce SK, Morris JF, Grusby MJ, Kaumaya P, van Buskirk A, Srinivasan M, et al. Antigen-presenting function of $B$ lymphocytes. Immunological reviews. 1988;106:149-80.

119. Rock KL, Reits E, Neefjes J. Present Yourself! By MHC Class I and MHC Class II Molecules. Trends in immunology. 2016;37(11):724-37.

120. Rodriguez-Pinto D. B cells as antigen presenting cells. Cellular immunology. 2005;238(2):67-75.

121. Molnarfi N, Schulze-Topphoff U, Weber MS, Patarroyo JC, Prod'homme T, Varrin-Doyer $\mathrm{M}$, et al. MHC class II-dependent B cell APC function is required for induction of CNS autoimmunity independent of myelin-specific antibodies. The Journal of experimental medicine. 2013;210(13):2921-37.

122. Rivera A, Chen CC, Ron N, Dougherty JP, Ron Y. Role of B cells as antigenpresenting cells in vivo revisited: antigen-specific $B$ cells are essential for $T$ cell expansion in lymph nodes and for systemic $T$ cell responses to low antigen concentrations. International immunology. 2001;13(12):1583-93.

123. Fraussen J, Claes N, Van Wijmeersch B, van Horssen J, Stinissen $P$, Hupperts $\mathrm{R}$, et al. B cells of multiple sclerosis patients induce autoreactive proinflammatory $\mathrm{T}$ cell responses. Clinical immunology (Orlando, $\mathrm{Fla}$ ). 2016;173:124-32.

124. Colombo M, Dono M, Gazzola P, Chiorazzi N, Mancardi G, Ferrarini M. Maintenance of $B$ lymphocyte-related clones in the cerebrospinal fluid of multiple sclerosis patients. European journal of immunology. 2003;33(12):3433-8.

125. Lovato L, Willis SN, Rodig SJ, Caron T, Almendinger SE, Howell OW, et al. Related $\mathrm{B}$ cell clones populate the meninges and parenchyma of patients with multiple sclerosis. Brain : a journal of neurology. 2011;134(Pt 2):534-41.

126. von Budingen HC, Kuo TC, Sirota M, van Belle CJ, Apeltsin L, Glanville J, et al. B cell exchange across the blood-brain barrier in multiple sclerosis. The Journal of clinical investigation. 2012;122(12):4533-43.

127. Cheng W, Chen G. Chemokines and chemokine receptors in multiple sclerosis. Mediators of inflammation. 2014;2014:659206.

128. Szczucinski A, Losy J. Chemokines and chemokine receptors in multiple sclerosis. Potential targets for new therapies. Acta neurologica Scandinavica. 2007;115(3):137-46.

129. Haas J, Bekeredjian-Ding I, Milkova M, Balint B, Schwarz A, Korporal M, et al. B cells undergo unique compartmentalized redistribution in multiple sclerosis. Journal of autoimmunity. 2011;37(4):289-99.

130. Mahad DJ, Howell SJ, Woodroofe MN. Expression of chemokines in the CSF and correlation with clinical disease activity in patients with multiple sclerosis. Journal of neurology, neurosurgery, and psychiatry. 2002;72(4):498-502.

131. Grogan JL, Ouyang W. A role for Th17 cells in the regulation of tertiary lymphoid follicles. European journal of immunology. 2012;42(9):2255-62.

132. Puthenparampil M, Federle L, Miante S, Zito A, Toffanin E, Ruggero S, et al. BAFF Index and CXCL13 levels in the cerebrospinal fluid associate respectively with intrathecal IgG synthesis and cortical atrophy in multiple sclerosis at clinical onset. Journal of neuroinflammation. 2017;14(1):11. 
133. Puthenparampil M, Miante S, Federle L, Zanetta C, Toffanin E, Ruggero S, et al. BAFF is decreased in the cerebrospinal fluid of multiple sclerosis at clinical onset. Journal of neuroimmunology. 2016;297:63-7.

134. Chu VT, Enghard P, Riemekasten G, Berek C. In vitro and in vivo activation induces BAFF and APRIL expression in B cells. Journal of immunology (Baltimore, Md : 1950). 2007;179(9):5947-57.

135. Dillon SR, Gross JA, Ansell SM, Novak AJ. An APRIL to remember: novel TNF ligands as therapeutic targets. Nature reviews Drug discovery. $2006 ; 5(3): 235-46$.

136. Krumbholz M, Theil D, Derfuss T, Rosenwald A, Schrader F, Monoranu CM, et al. BAFF is produced by astrocytes and up-regulated in multiple sclerosis lesions and primary central nervous system lymphoma. The Journal of experimental medicine. 2005;201(2):195-200.

137. Schneider P, MacKay F, Steiner V, Hofmann K, Bodmer JL, Holler N, et al. BAFF, a novel ligand of the tumor necrosis factor family, stimulates $B$ cell growth. The Journal of experimental medicine. 1999;189(11):1747-56.

138. Rahmanzadeh R, Weber MS, Bruck W, Navardi S, Sahraian MA. B cells in multiple sclerosis therapy-A comprehensive review. Acta neurologica Scandinavica. 2018;137(6):544-56.

139. Yokota HKKNT. Blood-brain barrier: A novel therapeutic target in multiple sclerosis. Clinical and Experimental Neuroimmunology 2015;6(2).

140. Alter A, Duddy M, Hebert S, Biernacki K, Prat A, Antel JP, et al. Determinants of human $B$ cell migration across brain endothelial cells. Journal of immunology (Baltimore, Md : 1950). 2003;170(9):4497-505.

141. Niino M, Bodner C, Simard ML, Alatab S, Gano D, Kim HJ, et al. Natalizumab effects on immune cell responses in multiple sclerosis. Annals of neurology. 2006;59(5):748-54.

142. Polman $\mathrm{CH}$, Reingold SC, Banwell B, Clanet $M$, Cohen JA, Filippi M, et al. Diagnostic criteria for multiple sclerosis: 2010 revisions to the McDonald criteria. Annals of neurology. 2011;69(2):292-302.

143. Cayrol R, Wosik K, Berard JL, Dodelet-Devillers A, Ifergan I, Kebir H, et al. Activated leukocyte cell adhesion molecule promotes leukocyte trafficking into the central nervous system. Nature immunology. 2008;9(2):137-45.

144. Thewissen M, Linsen L, Somers V, Geusens P, Raus J, Stinissen P. Premature immunosenescence in rheumatoid arthritis and multiple sclerosis patients. Ann N Y Acad Sci. 2005;1051:255-62.

145. Bruunsgaard $H$. The clinical impact of systemic low-level inflammation in elderly populations. With special reference to cardiovascular disease, dementia and mortality. Danish medical bulletin. 2006;53(3):285-309.

146. Krabbe KS, Pedersen M, Bruunsgaard H. Inflammatory mediators in the elderly. Experimental gerontology. 2004;39(5):687-99.

147. Salvioli S, Monti D, Lanzarini C, Conte M, Pirazzini C, Bacalini MG, et al. Immune system, cell senescence, aging and longevity--inflamm-aging reappraised. Current pharmaceutical design. 2013;19(9):1675-9.

148. Chan GK, Duque G. Age-related bone loss: old bone, new facts. Gerontology. 2002;48(2):62-71.

149. Riley RL. Impaired $B$ lymphopoiesis in old age: a role for inflammatory $B$ cells? Immunologic research. 2013;57(1-3):361-9.

150. Hakim FT, Gress RE. Immunosenescence: deficits in adaptive immunity in the elderly. Tissue Antigens. 2007;70(3):179-89. 
151. Gibson KL, Wu YC, Barnett Y, Duggan O, Vaughan R, Kondeatis E, et al. $\mathrm{B}$-cell diversity decreases in old age and is correlated with poor health status. Aging Cell. 2009;8(1):18-25.

152. Chong Y, Ikematsu H, Yamaji K, Nishimura M, Kashiwagi S, Hayashi J. Age-related accumulation of $\mathrm{Ig} \mathrm{V}(\mathrm{H})$ gene somatic mutations in peripheral B cells from aged humans. Clinical and experimental immunology. 2003;133(1):59-66.

153. Montoya-Ortiz G. Immunosenescence, aging, and systemic lupus erythematous. Autoimmune Dis. 2013;2013:267078.

154. Bolton $C$, Smith PA. The influence and impact of ageing and immunosenescence (ISC) on adaptive immunity during multiple sclerosis (MS) and the animal counterpart experimental autoimmune encephalomyelitis (EAE). Ageing research reviews. 2018;41:64-81.

155. Duszczyszyn DA, Williams JL, Mason H, Lapierre Y, Antel J, Haegert DG. Thymic involution and proliferative $\mathrm{T}$-cell responses in multiple sclerosis. Journal of neuroimmunology. 2010;221(1-2):73-80.

156. Haegert DG, Hackenbroch JD, Duszczyszyn D, Fitz-Gerald L, Zastepa E, Mason $\mathrm{H}$, et al. Reduced thymic output and peripheral naive CD4 T-cell alterations in primary progressive multiple sclerosis (PPMS). Journal of neuroimmunology. 2011;233(1-2):233-9.

157. Hug A, Korporal M, Schroder I, Haas J, Glatz K, Storch-Hagenlocher B, et al. Thymic export function and $\mathrm{T}$ cell homeostasis in patients with relapsing remitting multiple sclerosis. Journal of immunology (Baltimore, Md : 1950). 2003;171(1):432-7.

158. Rojas OL, Narváez CF, Greenberg HB, Angel J, Franco MA. Characterization of rotavirus specific $B$ cells and their relation with serological memory. Virology. 2008;380(2):234-42.

159. Rinaldi S, Pallikkuth S, George VK, de Armas LR, Pahwa R, Sanchez CM, et al. Paradoxical aging in HIV: immune senescence of $B$ Cells is most prominent in young age. Aging (Albany NY). 2017;9(4):1307-25.

160. Bulati M, Buffa S, Candore G, Caruso C, Dunn-Walters DK, Pellicano M, et al. B cells and immunosenescence: a focus on IgG+IgD-CD27- (DN) B cells in aged humans. Ageing research reviews. 2011;10(2):274-84.

161. Mahmood Z, Muhammad K, Schmalzing M, Roll P, Dörner T, Tony HP. CD27-IgD- memory $B$ cells are modulated by in vivo interleukin- 6 receptor (IL6R) blockade in rheumatoid arthritis. Arthritis Res Ther. 2015;17:61.

162. Wirths $S$, Lanzavecchia A. ABCB1 transporter discriminates human resting naive $B$ cells from cycling transitional and memory $B$ cells. European journal of immunology. 2005;35(12):3433-41.

163. Isnardi I, Ng YS, Menard L, Meyers G, Saadoun D, Srdanovic I, et al. Complement receptor 2/CD21- human naive B cells contain mostly autoreactive unresponsive clones. Blood. 2010;115(24):5026-36.

164. Wehr $C$, Eibel $H$, Masilamani $M$, Illges $H$, Schlesier $M$, Peter $H H$, et al. A new CD21low $B$ cell population in the peripheral blood of patients with SLE. Clinical immunology (Orlando, Fla). 2004;113(2):161-71.

165. Rakhmanov M, Keller B, Gutenberger S, Foerster C, Hoenig M, Driessen $G$, et al. Circulating CD21low B cells in common variable immunodeficiency resemble tissue homing, innate-like $B$ cells. Proceedings of the National Academy of Sciences of the United States of America. 2009;106(32):13451-6.

166. Saadoun D, Terrier B, Bannock J, Vazquez T, Massad C, Kang I, et al. Expansion of autoreactive unresponsive CD21-/low B cells in Sjogren's syndromeassociated lymphoproliferation. Arthritis and rheumatism. 2013;65(4):1085-96. 
167. Weiss GE, Crompton PD, Li S, Walsh LA, Moir S, Traore B, et al. Atypical memory $B$ cells are greatly expanded in individuals living in a malaria-endemic area. Journal of immunology (Baltimore, Md : 1950). 2009;183(3):2176-82.

168. Ehrhardt GR, Hijikata A, Kitamura H, Ohara O, Wang JY, Cooper MD. Discriminating gene expression profiles of memory $B$ cell subpopulations. The Journal of experimental medicine. 2008;205(8):1807-17.

169. Ehrhardt GR, Hsu JT, Gartland L, Leu CM, Zhang S, Davis RS, et al. Expression of the immunoregulatory molecule FcRH4 defines a distinctive tissuebased population of memory B cells. The Journal of experimental medicine. 2005;202(6):783-91.

170. Rubtsov AV, Rubtsova K, Fischer A, Meehan RT, Gillis JZ, Kappler JW, et al. Toll-like receptor 7 (TLR7)-driven accumulation of a novel CD11C(+) B-cell population is important for the development of autoimmunity. Blood. 2011;118(5):1305-15.

171. Hao Y, O'Neill P, Naradikian MS, Scholz JL, Cancro MP. A B-cell subset uniquely responsive to innate stimuli accumulates in aged mice. Blood. 2011;118(5):1294-304.

172. Rubtsova K, Rubtsov AV, Cancro MP, Marrack P. Age-Associated B Cells: A T-bet-Dependent Effector with Roles in Protective and Pathogenic Immunity. Journal of immunology (Baltimore, Md : 1950). 2015;195(5):1933-7.

173. Naradikian MS, Myles A, Beiting DP, Roberts KJ, Dawson L, Herati RS, et al. Cutting Edge: IL-4, IL-21, and IFN-gamma Interact To Govern T-bet and CD11C Expression in TLR-Activated B Cells. Journal of immunology (Baltimore, Md : 1950). 2016;197(4):1023-8.

174. Rubtsova K, Rubtsov AV, van Dyk LF, Kappler JW, Marrack P. T-box transcription factor T-bet, a key player in a unique type of B-cell activation essential for effective viral clearance. Proceedings of the National Academy of Sciences of the United States of America. 2013;110(34):E3216-24.

175. Rubtsova K, Rubtsov AV, Thurman JM, Mennona JM, Kappler JW, Marrack P. B cells expressing the transcription factor T-bet drive lupus-like autoimmunity. The Journal of clinical investigation. 2017;127(4):1392-404.

176. Domeier PP, Chodisetti SB, Soni C, Schell SL, Elias MJ, Wong EB, et al. IFN-gamma receptor and STAT1 signaling in B cells are central to spontaneous germinal center formation and autoimmunity. The Journal of experimental medicine. $2016 ; 213(5): 715-32$.

177. Peng SL, Szabo SJ, Glimcher LH. T-bet regulates IgG class switching and pathogenic autoantibody production. Proceedings of the National Academy of Sciences of the United States of America. 2002;99(8):5545-50.

178. Rubtsov AV, Marrack P, Rubtsova K. T-bet expressing B cells - Novel target for autoimmune therapies? Cellular immunology. 2017.

179. Wang Z, Wang Z, Wang J, Diao Y, Qian X, Zhu N. T-bet-Expressing B Cells Are Positively Associated with Crohn's Disease Activity and Support Th1 Inflammation. DNA Cell Biol. 2016;35(10):628-35.

180. Inglese $M$, Petracca $M$. Therapeutic strategies in multiple sclerosis: a focus on neuroprotection and repair and relevance to schizophrenia. Schizophrenia research. 2015;161(1):94-101.

181. European public assessment reports: European Medicines Agency 2017 [updated accessed 10/08/2018. Available from: http://www.ema.europa.eu/ema/index.jsp?curl=pages\%2Fmedicines\%2Flanding \%2Fepar search.jsp\&mid=WC0b01ac058001d124\&searchTab=\&alreadyLoaded =true\&isNewQuery=true\&status=Authorised\&status=Withdrawn\&status=Suspen 


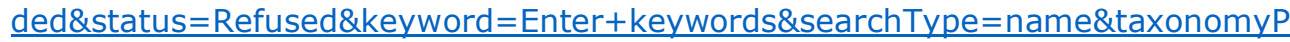
ath =Diseases. Nervous+System +Diseases. Autoimmune+Diseases +of +the +Nerv ous+System. Demyelinating+Autoimmune+Diseases $\% 2 C+C N S \&$ treeNumber $=\& C$ urrentCategory=Multiple+Sclerosis\&searchGenericType=generics.

182. Miller JR. The importance of early diagnosis of multiple sclerosis. Journal of managed care pharmacy : JMCP. 2004;10(3 Suppl B):S4-11.

183. Kavaliunas A, Manouchehrinia A, Stawiarz L, Ramanujam R, Agholme J, Hedstrom AK, et al. Importance of early treatment initiation in the clinical course of multiple sclerosis. Multiple sclerosis (Houndmills, Basingstoke, England). 2017;23(9):1233-40.

184. Kasper LH, Reder AT. Immunomodulatory activity of interferon-beta. Annals of clinical and translational neurology. 2014;1(8):622-31.

185. Shirani A, Zhao Y, Karim ME, Evans C, Kingwell E, van der Kop ML, et al. Association between use of interferon beta and progression of disability in patients with relapsing-remitting multiple sclerosis. Jama. 2012;308(3):247-56.

186. Plosker GL. Interferon-beta-1b: a review of its use in multiple sclerosis. CNS drugs. $2011 ; 25(1): 67-88$.

187. Bertolotto A. Evaluation of the impact of neutralizing antibodies on IFNbeta response. Clinica chimica acta; international journal of clinical chemistry. 2015;449:31-6.

188. Newsome SD, Kieseier BC, Liu S, You X, Kinter E, Hung S, et al. Peginterferon beta-1a reduces disability worsening in relapsing-remitting multiple sclerosis: 2-year results from ADVANCE. Therapeutic advances in neurological disorders. 2017;10(1):41-50.

189. Haas J, Korporal M, Balint B, Fritzsching B, Schwarz A, Wildemann B. Glatiramer acetate improves regulatory $T$-cell function by expansion of naive CD4(+)CD25(+)FOXP3(+)CD31(+) T-cells in patients with multiple sclerosis. Journal of neuroimmunology. 2009;216(1-2):113-7.

190. Neuhaus O, Farina C, Wekerle H, Hohlfeld R. Mechanisms of action of glatiramer acetate in multiple sclerosis. Neurology. 2001;56(6):702-8.

191. Comi G, Filippi M, Wolinsky JS. European/Canadian multicenter, doubleblind, randomized, placebo-controlled study of the effects of glatiramer acetate on magnetic resonance imaging--measured disease activity and burden in patients with relapsing multiple sclerosis. European/Canadian Glatiramer Acetate Study Group. Annals of neurology. 2001;49(3):290-7.

192. Interferon beta- $1 \mathrm{~b}$ in the treatment of multiple sclerosis: final outcome of the randomized controlled trial. The IFNB Multiple Sclerosis Study Group and The University of British Columbia MS/MRI Analysis Group. Neurology. 1995;45(7):1277-85.

193. Johnson KP, Brooks BR, Cohen JA, Ford CC, Goldstein J, Lisak RP, et al. Copolymer 1 reduces relapse rate and improves disability in relapsing-remitting multiple sclerosis: results of a phase III multicenter, double-blind placebocontrolled trial. The Copolymer 1 Multiple Sclerosis Study Group. Neurology. $1995 ; 45(7): 1268-76$.

194. Soelberg Sorensen P. Safety concerns and risk management of multiple sclerosis therapies. Acta neurologica Scandinavica. 2017;136(3):168-86.

195. Walther EU, Hohlfeld R. Multiple sclerosis: side effects of interferon beta therapy and their management. Neurology. 1999;53(8):1622-7.

196. Bar-Or A, Pachner A, Menguy-Vacheron F, Kaplan J, Wiendl H. Teriflunomide and its mechanism of action in multiple sclerosis. Drugs. $2014 ; 74(6): 659-74$. 
197. O'Connor P, Comi G, Freedman MS, Miller AE, Kappos L, Bouchard JP, et al. Long-term safety and efficacy of teriflunomide: Nine-year follow-up of the randomized TEMSO study. Neurology. 2016;86(10):920-30.

198. O'Connor P, Wolinsky JS, Confavreux C, Comi G, Kappos L, Olsson TP, et al. Randomized trial of oral teriflunomide for relapsing multiple sclerosis. The New England journal of medicine. 2011;365(14):1293-303.

199. Gross CC, Schulte-Mecklenbeck A, Klinsing S, Posevitz-Fejfar A, Wiendl H, Klotz L. Dimethyl fumarate treatment alters circulating $T$ helper cell subsets in multiple sclerosis. Neurology(R) neuroimmunology \& neuroinflammation. 2016;3(1):e183.

200. Linker RA, Lee DH, Ryan S, van Dam AM, Conrad R, Bista P, et al. Fumaric acid esters exert neuroprotective effects in neuroinflammation via activation of the Nrf2 antioxidant pathway. Brain : a journal of neurology. 2011;134(Pt 3):67892.

201. Fox RJ, Miller DH, Phillips JT, Hutchinson M, Havrdova E, Kita M, et al. Placebo-controlled phase 3 study of oral BG-12 or glatiramer in multiple sclerosis. The New England journal of medicine. 2012;367(12):1087-97.

202. Gold R, Kappos L, Arnold DL, Bar-Or A, Giovannoni G, Selmaj K, et al. Placebo-controlled phase 3 study of oral BG-12 for relapsing multiple sclerosis. The New England journal of medicine. 2012;367(12):1098-107.

203. Kita M, Fox RJ, Gold R, Giovannoni G, Phillips JT, Sarda SP, et al. Effects of delayed-release dimethyl fumarate (DMF) on health-related quality of life in patients with relapsing-remitting multiple sclerosis: an integrated analysis of the phase 3 DEFINE and CONFIRM studies. Clinical therapeutics. 2014;36(12):195871.

204. Cohen JA, Coles AJ, Arnold DL, Confavreux C, Fox EJ, Hartung HP, et al. Alemtuzumab versus interferon beta 1 a as first-line treatment for patients with relapsing-remitting multiple sclerosis: a randomised controlled phase 3 trial. Lancet (London, England). 2012;380(9856):1819-28.

205. Willis MD, Harding KE, Pickersgill TP, Wardle M, Pearson OR, Scolding NJ, et al. Alemtuzumab for multiple sclerosis: Long term follow-up in a multi-centre cohort. Multiple sclerosis (Houndmills, Basingstoke, England). 2016;22(9):121523.

206. Havrdova E, Horakova D, Kovarova I. Alemtuzumab in the treatment of multiple sclerosis: key clinical trial results and considerations for use. Therapeutic advances in neurological disorders. 2015;8(1):31-45.

207. Sheremata WA, Minagar A, Alexander JS, Vollmer T. The role of alpha-4 integrin in the aetiology of multiple sclerosis: current knowledge and therapeutic implications. CNS drugs. 2005;19(11):909-22.

208. Miller DH, Khan OA, Sheremata WA, Blumhardt LD, Rice GP, Libonati MA, et al. A controlled trial of natalizumab for relapsing multiple sclerosis. The New England journal of medicine. 2003;348(1):15-23.

209. Polman $\mathrm{CH}, \mathrm{O}$ 'Connor PW, Havrdova E, Hutchinson M, Kappos L, Miller DH, et al. A randomized, placebo-controlled trial of natalizumab for relapsing multiple sclerosis. The New England journal of medicine. 2006;354(9):899-910.

210. Choi JW, Gardell SE, Herr DR, Rivera R, Lee CW, Noguchi K, et al. FTY720 (fingolimod) efficacy in an animal model of multiple sclerosis requires astrocyte sphingosine 1-phosphate receptor 1 (S1P1) modulation. Proceedings of the National Academy of Sciences of the United States of America. 2011;108(2):7516. 
211. Claes N, Dhaeze T, Fraussen J, Broux B, Van Wijmeersch B, Stinissen P, et al. Compositional changes of $B$ and $T$ cell subtypes during fingolimod treatment in multiple sclerosis patients: a 12-month follow-up study. Plos one. 2014;9(10):e111115.

212. Kappos L, Radue EW, O'Connor P, Polman C, Hohlfeld R, Calabresi P, et al. A placebo-controlled trial of oral fingolimod in relapsing multiple sclerosis. The New England journal of medicine. 2010;362(5):387-401.

213. Bielekova B. Daclizumab therapy for multiple sclerosis. Neurotherapeutics : the journal of the American Society for Experimental NeuroTherapeutics. 2013;10(1):55-67.

214. Avasarala J. DRESS Syndrome and Daclizumab Failure-Were Potentially Dangerous Signs Missed in Clinical Trials? Drug target insights. 2018;12:1177392818785136.

215. Ocrelizumab for multiple sclerosis. Drug and therapeutics bulletin. 2018;56(7):80-4.

216. Tarek Gaber FRCP CSM. Cladribine tablets and multiple sclerosis: NICE technology appraisal Progress in Neurology and Psychiatry. 2018;22(1).

217. Kappos L, Bar-Or A, Cree BAC, Fox RJ, Giovannoni G, Gold R, et al. Siponimod versus placebo in secondary progressive multiple sclerosis (EXPAND): a double-blind, randomised, phase 3 study. Lancet (London, England). 2018;391(10127):1263-73.

218. Rasche L, Paul F. Ozanimod for the treatment of relapsing remitting multiple sclerosis. Expert opinion on pharmacotherapy. 2018:1-14.

219. Mao-Draayer Y, Sarazin J, Fox D, Schiopu E. The sphingosine-1-phosphate receptor: A novel therapeutic target for multiple sclerosis and other autoimmune diseases. Clinical immunology (Orlando, Fla). 2017;175:10-5.

220. Pierpont TM, Limper CB, Richards KL. Past, Present, and Future of Rituximab-The World's First Oncology Monoclonal Antibody Therapy. Frontiers in oncology. 2018;8:163.

221. Gelfand JM, Cree BAC, Hauser SL. Ocrelizumab and Other CD20(+) B-CellDepleting Therapies in Multiple Sclerosis. Neurotherapeutics : the journal of the American Society for Experimental NeuroTherapeutics. 2017;14(4):835-41.

222. Pastuszak Z, Stepien A, Tomczykiewicz K, Piusinska-Macoch R, DurkaKesy M. [Mitoxantrone role in treatment of primary progressive multiple sclerosis]. Polski merkuriusz lekarski : organ Polskiego Towarzystwa Lekarskiego. 2016;40(235):66-9.

223. Squibb BM. Bristol Myers Squibb Receives Positive CHMP Opinion

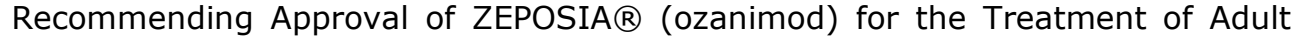
Patients with Relapsing Remitting Multiple Sclerosis with Active Disease

224. \#AAN2018 - ALKS 8700 Shows Promise as RRMS Therapy, Phase 3 Trial Interim Results Suggest [press release]. Accessed 0412182018.

225. Merck Announces Positive Phase IIB Results for Evobrutinib in Relapsing Multiple Sclerosis [press release]. Accessed 0412182018.

226. A Phase 3, Randomized, Multi-center, Double-blinded, Active-controlled Study to Assess the Efficacy and Safety/Tolerability of Ublituximab (TG-1101; UTX) as Compared to Teriflunomide in Subjects With Relapsing Multiple Sclerosis (RMS) (ULTIMATE 1) [Internet]. 2018 [cited Accessed 041218]. Available from: https://clinicaltrials.gov/ct2/show/NCT03277261?term=ublituximab\&rank=3. 
227. Ublituximab (TGTX-1101) [updated Accessed 041218. Available from: https://multiplesclerosisnewstoday.com/experimental-treatments-for-

ms/ublituximab-tgtx-1101/.

228. Efficacy and Safety of Ofatumumab Compared to Teriflunomide in Patients With Relapsing Multiple Sclerosis (ASCLEPIOS I) [Internet]. 2018 [cited Accessed 041218]. Available from: https://clinicaltrials.gov/ct2/show/NCT02792218?cond=ofatumumab\&rank=3.

229. Hauser $S$ et al. AAN 2017. Abstract S16.005. 2017.

230. A. M. Glatiramer Acetate Depot (extended-release) Phase IIA study in patients with Relapsing Remitting Multiple Sclerosis: Six months' interim analysis. ECTRIMS Online Library Abstract. 2017.

231. ATX-MS-1467 in Multiple Sclerosis [Internet]. 2017 [cited Accessed 041218]. Available from: https://clinicaltrials.gov/ct2/show/NCT01973491.

232. Chataway J, Martin K, Barrell K, Sharrack B, Stolt P, Wraith DC. Effects of ATX-MS-1467 immunotherapy over 16 weeks in relapsing multiple sclerosis. Neurology. 2018;90(11):e955-e62.

233. ATL1102 [Internet]. Available from: https://www.anzctr.org.au/Trial/Registration/TrialReview.aspx?id=82556.

234. FDA Clears Antisense Therapeutics to Proceed with Phase $2 \mathrm{~b}$ Trial of ATL1102 in RRMS and SPMS Patients. 2017.

235. Sanofi, Principia agree to develop multiple sclerosis drug candidate [press release]. 2017.

236. Mrowietz U, Altmeyer P, Bieber T, Rocken M, Schopf RE, Sterry W. Treatment of psoriasis with fumaric acid esters (Fumaderm). Journal der Deutschen Dermatologischen Gesellschaft = Journal of the German Society of Dermatology : JDDG. 2007;5(8):716-7.

237. Mrowietz U, Christophers E, Altmeyer P. Treatment of psoriasis with fumaric acid esters: results of a prospective multicentre study. German Multicentre Study. The British journal of dermatology. 1998;138(3):456-60.

238. Prinz JC. The role of T cells in psoriasis. Journal of the European Academy of Dermatology and Venereology : JEADV. 2003;17(3):257-70.

239. Schweckendiek W. [Treatment of psoriasis vulgaris]. Medizinische Monatsschrift. 1959;13(2):103-4.

240. Bovenschen HJ, Langewouters AM, van de Kerkhof PC. Dimethylfumarate for psoriasis: Pronounced effects on lesional T-cell subsets, epidermal proliferation and differentiation, but not on natural killer T cells in immunohistochemical study. American journal of clinical dermatology. 2010;11(5):343-50.

241. Ockenfels HM, Schultewolter T, Ockenfels G, Funk R, Goos M. The antipsoriatic agent dimethylfumarate immunomodulates $\mathrm{T}$-cell cytokine secretion and inhibits cytokines of the psoriatic cytokine network. The British journal of dermatology. 1998;139(3):390-5.

242. Reich K, Thaci D, Mrowietz U, Kamps A, Neureither M, Luger T. Efficacy and safety of fumaric acid esters in the long-term treatment of psoriasis--a retrospective study (FUTURE). Journal der Deutschen Dermatologischen Gesellschaft $=$ Journal of the German Society of Dermatology : JDDG. 2009;7(7):603-11.

243. Schimrigk S, Brune N, Hellwig K, Lukas C, Bellenberg B, Rieks M, et al. Oral fumaric acid esters for the treatment of active multiple sclerosis: an openlabel, baseline-controlled pilot study. European journal of neurology. 2006;13(6):604-10. 
244. Litjens NH, van Strijen E, van Gulpen C, Mattie H, van Dissel JT, Thio HB, et al. In vitro pharmacokinetics of anti-psoriatic fumaric acid esters. BMC pharmacology. 2004;4:22.

245. Litjens $\mathrm{NH}$, Burggraaf J, van Strijen $\mathrm{E}$, van Gulpen C, Mattie $\mathrm{H}$, Schoemaker RC, et al. Pharmacokinetics of oral fumarates in healthy subjects. British journal of clinical pharmacology. 2004;58(4):429-32.

246. Rostami-Yazdi M, Clement B, Mrowietz U. Pharmacokinetics of antipsoriatic fumaric acid esters in psoriasis patients. Archives of Dermatological Research. 2010;302(7):531-8.

247. Rostami-Yazdi M, Clement B, Schmidt TJ, Schinor D, Mrowietz U. Detection of metabolites of fumaric acid esters in human urine: implications for their mode of action. The Journal of investigative dermatology. $2009 ; 129(1): 231$ 4.

248. Schmidt TJ, Ak M, Mrowietz U. Reactivity of dimethyl fumarate and methylhydrogen fumarate towards glutathione and $\mathrm{N}$-acetyl-L-cysteine-preparation of S-substituted thiosuccinic acid esters. Bioorganic \& medicinal chemistry. 2007;15(1):333-42.

249. Ghashghaeinia M, Bobbala D, Wieder T, Koka S, Bruck J, Fehrenbacher B, et al. Targeting glutathione by dimethylfumarate protects against experimental malaria by enhancing erythrocyte cell membrane scrambling. American journal of physiology Cell physiology. 2010;299(4):C791-804.

250. Sheikh SI, Nestorov I, Russell H, O'Gorman J, Huang R, Milne GL, et al. Tolerability and pharmacokinetics of delayed-release dimethyl fumarate administered with and without aspirin in healthy volunteers. Clinical therapeutics. 2013;35(10):1582-94.e9.

251. Gillard GO, Collette B, Anderson J, Chao J, Scannevin RH, Huss DJ, et al. DMF, but not other fumarates, inhibits NF-kappaB activity in vitro in an Nrf2independent manner. Journal of neuroimmunology. 2015;283:74-85.

252. Moi P, Chan K, Asunis I, Cao A, Kan YW. Isolation of NF-E2-related factor 2 (Nrf2), a NF-E2-like basic leucine zipper transcriptional activator that binds to the tandem NF-E2/AP1 repeat of the beta-globin locus control region. Proceedings of the National Academy of Sciences of the United States of America. 1994;91(21):9926-30.

253. Battino M, Giampieri F, Pistollato F, Sureda A, de Oliveira MR, Pittala V, et al. Nrf2 as regulator of innate immunity: A molecular Swiss army knife! Biotechnology advances. 2018;36(2):358-70.

254. Benarroch EE. Nrf2, cellular redox regulation, and neurologic implications. Neurology. 2017;88(20):1942-50.

255. Brennan MS, Matos MF, Li B, Hronowski X, Gao B, Juhasz P, et al. Dimethyl fumarate and monoethyl fumarate exhibit differential effects on KEAP1, NRF2 activation, and glutathione depletion in vitro. PloS one. 2015;10(3):e0120254.

256. Kihara Y, Groves A, Rivera RR, Chun J. Dimethyl fumarate inhibits integrin alpha4 expression in multiple sclerosis models. Annals of clinical and translational neurology. $2015 ; 2(10): 978-83$.

257. Schulze-Topphoff U, Varrin-Doyer M, Pekarek K, Spencer CM, Shetty A, Sagan SA, et al. Dimethyl fumarate treatment induces adaptive and innate immune modulation independent of Nrf2. Proceedings of the National Academy of Sciences of the United States of America. 2016;113(17):4777-82.

258. Blewett MM, Xie J, Zaro BW, Backus KM, Altman A, Teijaro JR, et al. Chemical proteomic map of dimethyl fumarate-sensitive cysteines in primary human T cells. Science signaling. 2016;9(445):rs10. 
259. Gerdes S, Shakery K, Mrowietz U. Dimethylfumarate inhibits nuclear binding of nuclear factor kappaB but not of nuclear factor of activated $T$ cells and CCAAT/enhancer binding protein beta in activated human $T$ cells. The British journal of dermatology. 2007;156(5):838-42.

260. Chen H, Assmann JC, Krenz A, Rahman M, Grimm M, Karsten CM, et al. Hydroxycarboxylic acid receptor 2 mediates dimethyl fumarate's protective effect in EAE. The Journal of clinical investigation. 2014;124(5):2188-92.

261. Benyo Z, Gille A, Kero J, Csiky M, Suchankova MC, Nusing RM, et al. GPR109A (PUMA-G/HM74A) mediates nicotinic acid-induced flushing. The Journal of clinical investigation. 2005;115(12):3634-40.

262. Kostylina G, Simon D, Fey MF, Yousefi S, Simon HU. Neutrophil apoptosis mediated by nicotinic acid receptors (GPR109A). Cell death and differentiation. 2008;15(1):134-42.

263. Maciejewski-Lenoir D, Richman JG, Hakak Y, Gaidarov I, Behan DP, Connolly DT. Langerhans cells release prostaglandin D2 in response to nicotinic acid. The Journal of investigative dermatology. 2006;126(12):2637-46.

264. Offermanns S. Hydroxy-Carboxylic Acid Receptor Actions in Metabolism. Trends in endocrinology and metabolism: TEM. 2017;28(3):227-36.

265. Offermanns S, Schwaninger M. Nutritional or pharmacological activation of $\mathrm{HCA}(2)$ ameliorates neuroinflammation. Trends in molecular medicine. 2015;21(4):245-55.

266. Rahman M, Muhammad S, Khan MA, Chen H, Ridder DA, Muller-Fielitz H, et al. The beta-hydroxybutyrate receptor HCA2 activates a neuroprotective subset of macrophages. Nature communications. 2014;5:3944.

267. Parodi B, Rossi S, Morando S, Cordano C, Bragoni A, Motta C, et al. Fumarates modulate microglia activation through a novel HCAR2 signaling pathway and rescue synaptic dysregulation in inflamed CNS. Acta neuropathologica. 2015;130(2):279-95.

268. Digby JE, Martinez F, Jefferson A, Ruparelia N, Chai J, Wamil M, et al. Antiinflammatory effects of nicotinic acid in human monocytes are mediated by GPR109A dependent mechanisms. Arteriosclerosis, thrombosis, and vascular biology. 2012;32(3):669-76.

269. Zandi-Nejad K, Takakura A, Jurewicz M, Chandraker AK, Offermanns S, Mount D, et al. The role of HCA2 (GPR109A) in regulating macrophage function. FASEB journal : official publication of the Federation of American Societies for Experimental Biology. 2013;27(11):4366-74.

270. Lu SC. Glutathione synthesis. Biochimica et biophysica acta. 2013;1830(5):3143-53.

271. Dibbert S, Clement B, Skak-Nielsen T, Mrowietz U, Rostami-Yazdi M. Detection of fumarate-glutathione adducts in the portal vein blood of rats: evidence for rapid dimethylfumarate metabolism. Arch Dermatol Res. 2013;305(5):447-51.

272. Lehmann JC, Listopad JJ, Rentzsch CU, Igney FH, von Bonin A, Hennekes $\mathrm{HH}$, et al. Dimethylfumarate induces immunosuppression via glutathione depletion and subsequent induction of heme oxygenase 1 . The Journal of investigative dermatology. 2007;127(4):835-45.

273. Albrecht P, Bouchachia I, Goebels N, Henke N, Hofstetter HH, Issberner $A$, et al. Effects of dimethyl fumarate on neuroprotection and immunomodulation. Journal of neuroinflammation. 2012;9:163. 
274. Huang H, Taraboletti A, Shriver LP. Dimethyl fumarate modulates antioxidant and lipid metabolism in oligodendrocytes. Redox biology. 2015;5:16975.

275. Diebold M, Sievers C, Bantug G, Sanderson N, Kappos L, Kuhle J, et al. Dimethyl fumarate influences innate and adaptive immunity in multiple sclerosis. Journal of autoimmunity. 2018;86:39-50.

276. Kornberg MD, Bhargava P, Kim PM, Putluri V, Snowman AM, Putluri N, et al. Dimethyl fumarate targets GAPDH and aerobic glycolysis to modulate immunity. Science (New York, NY). 2018;360(6387):449-53.

277. Shi LZ, Wang R, Huang G, Vogel P, Neale G, Green DR, et al. HIF1alphadependent glycolytic pathway orchestrates a metabolic checkpoint for the differentiation of $\mathrm{TH} 17$ and Treg cells. The Journal of experimental medicine. $2011 ; 208(7): 1367-76$.

278. Vats D, Mukundan L, Odegaard JI, Zhang L, Smith KL, Morel CR, et al. Oxidative metabolism and PGC-1beta attenuate macrophage-mediated inflammation. Cell metabolism. 2006;4(1):13-24.

279. Schilling S, Goelz S, Linker R, Luehder F, Gold R. Fumaric acid esters are effective in chronic experimental autoimmune encephalomyelitis and suppress macrophage infiltration. Clinical and experimental immunology. 2006;145(1):101-7.

280. Demir S, Heckers S, Pedreiturria X, Hess D, Trampe AK, Chan A, et al. Low dose fumaric acid esters are effective in a mouse model of spontaneous chronic encephalomyelitis. Journal of neuroimmunology. 2015;285:16-21.

281. Reick C, Ellrichmann G, Thone J, Scannevin RH, Saft C, Linker RA, et al. Neuroprotective dimethyl fumarate synergizes with immunomodulatory interferon beta to provide enhanced axon protection in autoimmune neuroinflammation. Experimental neurology. 2014;257:50-6.

282. Biogen. History accessed 14 june 2018 [Available from: https://www.biogen.com/en us/history-overview.html.

283. Kappos L, Gold R, Miller DH, Macmanus DG, Havrdova E, Limmroth V, et al. Efficacy and safety of oral fumarate in patients with relapsing-remitting multiple sclerosis: a multicentre, randomised, double-blind, placebo-controlled phase IIb study. Lancet (London, England). 2008;372(9648):1463-72.

284. Gold R, Giovannoni G, Phillips JT, Fox RJ, Zhang A, Meltzer L, et al. Efficacy and safety of delayed-release dimethyl fumarate in patients newly diagnosed with relapsing-remitting multiple sclerosis (RRMS). Multiple sclerosis (Houndmills, Basingstoke, England). 2015;21(1):57-66.

285. Giovannoni G, Gold R, Kappos L, Arnold DL, Bar-Or A, Marantz JL, et al. Delayed-release dimethyl fumarate and disability assessed by the Multiple Sclerosis Functional Composite: Integrated analysis of DEFINE and CONFIRM. Multiple sclerosis journal - experimental, translational and clinical. 2016;2:2055217316634111.

286. Gold R, Arnold DL, Bar-Or A, Hutchinson M, Kappos L, Havrdova E, et al. Long-term effects of delayed-release dimethyl fumarate in multiple sclerosis: Interim analysis of ENDORSE, a randomized extension study. Multiple sclerosis (Houndmills, Basingstoke, England). 2017;23(2):253-65.

287. Baharnoori M, Lyons J, Dastagir A, Koralnik I, Stankiewicz JM. Nonfatal $\mathrm{PML}$ in a patient with multiple sclerosis treated with dimethyl fumarate. Neurology(R) neuroimmunology \& neuroinflammation. 2016;3(5):e274.

288. Zoghi S, Amirghofran Z, Nikseresht A, Ashjazadeh N, Kamali-Sarvestani $\mathrm{E}$, Rezaei N. Cytokine secretion pattern in treatment of lymphocytes of multiple 
sclerosis patients with fumaric acid esters. Immunological investigations. 2011;40(6):581-96.

289. Kivisakk P, Mahad DJ, Callahan MK, Sikora K, Trebst C, Tucky B, et al. Expression of CCR7 in multiple sclerosis: implications for CNS immunity. Annals of neurology. 2004;55(5):627-38.

290. MacLeod MK, Kappler JW, Marrack P. Memory CD4 T cells: generation, reactivation and re-assignment. Immunology. 2010;130(1):10-5.

291. Wulff H, Calabresi PA, Allie R, Yun S, Pennington M, Beeton C, et al. The voltage-gated $\mathrm{Kv} 1.3 \mathrm{~K}(+)$ channel in effector memory $\mathrm{T}$ cells as new target for MS. The Journal of clinical investigation. 2003;111(11):1703-13.

292. Gjelstrup MC, Stilund M, Petersen T, Moller HJ, Petersen EL, Christensen T. Subsets of activated monocytes and markers of inflammation in incipient and progressed multiple sclerosis. Immunology and cell biology. 2018;96(2):160-74. 293. Glennon-Alty L, Hackett AP, Chapman EA, Wright HL. Neutrophils and redox stress in the pathogenesis of autoimmune disease. Free radical biology \& medicine. 2018.

294. Naegele M, Tillack K, Reinhardt S, Schippling S, Martin R, Sospedra M. Neutrophils in multiple sclerosis are characterized by a primed phenotype. Journal of neuroimmunology. 2012;242(1-2):60-71.

295. Li R, Rezk A, Ghadiri M, Luessi F, Zipp F, Li H, et al. Dimethyl Fumarate Treatment Mediates an Anti-Inflammatory Shift in B Cell Subsets of Patients with Multiple Sclerosis. Journal of immunology (Baltimore, Md : 1950). 2017;198(2):691-8.

296. Thangarajh M, Masterman T, Hillert J, Moerk S, Jonsson R. A proliferationinducing ligand (APRIL) is expressed by astrocytes and is increased in multiple sclerosis. Scandinavian journal of immunology. 2007;65(1):92-8.

297. Amato MP, Zipoli V, Portaccio E. Multiple sclerosis-related cognitive changes: a review of cross-sectional and longitudinal studies. Journal of the neurological sciences. $2006 ; 245(1-2): 41-6$.

298. Macias Islas MA, Ciampi E. Assessment and Impact of Cognitive Impairment in Multiple Sclerosis: An Overview. Biomedicines. 2019;7(1).

299. Rao SM. Neuropsychological Screening Battery for Multiple Sclerosis;. National Multiple Sclerosis Society:

New York. 1991.

300. Fleischer V, Friedrich M, Rezk A, Buhler U, Witsch E, Uphaus T, et al. Treatment response to dimethyl fumarate is characterized by disproportionate $\mathrm{CD} 8+\mathrm{T}$ cell reduction in MS. Multiple sclerosis (Houndmills, Basingstoke, England). 2017:1352458517703799.

301. Ghadiri M, Rezk A, Li R, Evans A, Luessi F, Zipp F, et al. Dimethyl fumarate-induced lymphopenia in MS due to differential T-cell subset apoptosis. Neurology(R) neuroimmunology \& neuroinflammation. 2017;4(3):e340.

302. Khatri BO, Garland J, Berger J, Kramer J, Sershon L, Olapo T, et al. The effect of dimethyl fumarate (Tecfidera) on lymphocyte counts: A potential contributor to progressive multifocal leukoencephalopathy risk. Multiple sclerosis and related disorders. 2015;4(4):377-9.

303. Spencer CM, Crabtree-Hartman EC, Lehmann-Horn K, Cree BA, Zamvil SS. Reduction of CD8(+) T lymphocytes in multiple sclerosis patients treated with dimethyl fumarate. Neurology(R) neuroimmunology \& neuroinflammation. 2015;2(3):e76.

304. Medina S, Villarrubia N, Sainz de la Maza S, Lifante J, Costa-Frossard L,

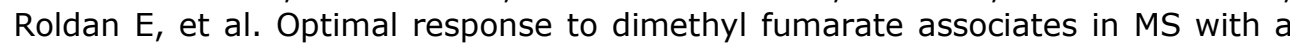


shift from an inflammatory to a tolerogenic blood cell profile. Multiple sclerosis (Houndmills, Basingstoke, England). 2017:1352458517717088.

305. Chaves C, Ganguly R, Ceresia C, Camac A. Lymphocyte subtypes in relapsing-remitting multiple sclerosis patients treated with dimethyl fumarate. Multiple sclerosis journal - experimental, translational and clinical. 2017;3(2):2055217317702933.

306. Bielekova B, Catalfamo M, Reichert-Scrivner S, Packer A, Cerna M, Waldmann TA, et al. Regulatory CD56(bright) natural killer cells mediate immunomodulatory effects of IL-2Ralpha-targeted therapy (daclizumab) in multiple sclerosis. Proceedings of the National Academy of Sciences of the United States of America. 2006;103(15):5941-6.

307. Peng H, Guerau-de-Arellano M, Mehta VB, Yang Y, Huss DJ, Papenfuss TL, et al. Dimethyl fumarate inhibits dendritic cell maturation via nuclear factor kappaB (NF-kappaB) and extracellular signal-regulated kinase 1 and 2 (ERK1/2) and mitogen stress-activated kinase 1 (MSK1) signaling. The Journal of biological chemistry. 2012;287(33):28017-26.

308. Longbrake EE, Ramsbottom MJ, Cantoni C, Ghezzi L, Cross AH, Piccio L. Dimethyl fumarate selectively reduces memory $T$ cells in multiple sclerosis patients. Multiple sclerosis (Houndmills, Basingstoke, England). 2016;22(8):1061-70.

309. Wu Q, Wang Q, Mao G, Dowling CA, Lundy SK, Mao-Draayer Y. Dimethyl Fumarate Selectively Reduces Memory $T$ Cells and Shifts the Balance between Th1/Th17 and Th2 in Multiple Sclerosis Patients. Journal of immunology (Baltimore, Md : 1950). 2017;198(8):3069-80.

310. Smith MD, Martin KA, Calabresi PA, Bhargava P. Dimethyl fumarate alters $B$-cell memory and cytokine production in MS patients. Annals of clinical and translational neurology. 2017;4(5):351-5.

311. Lundy SK, Wu Q, Wang Q, Dowling CA, Taitano SH, Mao G, et al. Dimethyl fumarate treatment of relapsing-remitting multiple sclerosis influences $B$-cell subsets. Neurology(R) neuroimmunology \& neuroinflammation. 2016;3(2):e211. 312. Longbrake EE, Cantoni C, Chahin S, Cignarella F, Cross AH, Piccio L. Dimethyl fumarate induces changes in B- and T-lymphocyte function independent of the effects on absolute lymphocyte count. Multiple sclerosis (Houndmills, Basingstoke, England). 2017:1352458517707069.

313. Bar-Or A, Oliveira EM, Anderson DE, Krieger JI, Duddy M, O'Connor KC, et al. Immunological memory: contribution of memory $B$ cells expressing costimulatory molecules in the resting state. Journal of immunology (Baltimore, Md : 1950). 2001;167(10):5669-77.

314. Good KL, Avery DT, Tangye SG. Resting human memory B cells are intrinsically programmed for enhanced survival and responsiveness to diverse stimuli compared to naive B cells. Journal of immunology (Baltimore, Md : 1950). 2009;182(2):890-901.

315. Lehmann-Horn K, Kinzel S, Weber MS. Deciphering the Role of B Cells in Multiple Sclerosis-Towards Specific Targeting of Pathogenic Function. International journal of molecular sciences. 2017;18(10).

316. Montes Diaz G, Fraussen J, Van Wijmeersch B, Hupperts R, Somers V. Dimethyl fumarate induces a persistent change in the composition of the innate and adaptive immune system in multiple sclerosis patients. Scientific reports. 2018;8(1):8194. 
317. Montes Diaz G, Hupperts R, Fraussen J, Somers V. Dimethyl fumarate treatment in multiple sclerosis: Recent advances in clinical and immunological studies. Autoimmunity reviews. 2018;17(12):1240-50.

318. de Jong R, Bezemer AC, Zomerdijk TP, van de Pouw-Kraan T, Ottenhoff $\mathrm{TH}$, Nibbering PH. Selective stimulation of T helper 2 cytokine responses by the anti-psoriasis agent monomethylfumarate. European journal of immunology. 1996;26(9):2067-74.

319. Treumer F, Zhu K, Glaser R, Mrowietz U. Dimethylfumarate is a potent inducer of apoptosis in human $T$ cells. The Journal of investigative dermatology. 2003;121(6):1383-8.

320. Invitrogen TF. eBioscience Annexin V Apoptosis Detection Kit FITC. 2017. 321. Warnatz K, Salzer U, Rizzi M, Fischer B, Gutenberger S, Bohm J, et al. Bcell activating factor receptor deficiency is associated with an adult-onset antibody deficiency syndrome in humans. Proceedings of the National Academy of Sciences of the United States of America. 2009;106(33):13945-50.

322. Huntington ND, Tomioka R, Clavarino C, Chow AM, Linares D, Mana P, et al. A BAFF antagonist suppresses experimental autoimmune encephalomyelitis by targeting cell-mediated and humoral immune responses. International immunology. 2006;18(10):1473-85.

323. Hartung HP, Kieseier BC. Atacicept: targeting B cells in multiple sclerosis. Therapeutic advances in neurological disorders. 2010;3(4):205-16.

324. Thangarajh M, Gomes A, Masterman T, Hillert J, Hjelmstrom P. Expression of B-cell-activating factor of the TNF family (BAFF) and its receptors in multiple sclerosis. Journal of neuroimmunology. 2004;152(1-2):183-90.

325. Ireland SJ, Blazek M, Harp CT, Greenberg B, Frohman EM, Davis LS, et al. Antibody-independent $B$ cell effector functions in relapsing remitting multiple sclerosis: clues to increased inflammatory and reduced regulatory B cell capacity. Autoimmunity. 2012;45(5):400-14.

326. Kosmaczewska A, Bilinska M, Ciszak L, Noga L, Pawlak E, Szteblich A, et al. Different patterns of activation markers expression and CD4+ T-cell responses to ex vivo stimulation in patients with clinically quiescent multiple sclerosis (MS). Journal of neuroimmunology. 2007;189(1-2):137-46.

327. Schloder J, Berges C, Luessi F, Jonuleit H. Dimethyl Fumarate Therapy Significantly Improves the Responsiveness of T Cells in Multiple Sclerosis Patients for Immunoregulation by Regulatory $T$ Cells. International journal of molecular sciences. $2017 ; 18(2)$.

328. Flores-Borja F, Bosma A, Ng D, Reddy V, Ehrenstein MR, Isenberg DA, et al. CD19+CD24hiCD38hi B cells maintain regulatory T cells while limiting TH1 and TH17 differentiation. Science translational medicine. 2013;5(173):173ra23.

329. Staun-Ram E, Najjar E, Volkowich A, Miller A. Dimethyl fumarate as a firstvs second-line therapy in MS: Focus on B cells. Neurology $(R)$ neuroimmunology \& neuroinflammation. 2018;5(6):e508.

330. Serafini B, Rosicarelli B, Magliozzi R, Stigliano E, Aloisi F. Detection of ectopic B-cell follicles with germinal centers in the meninges of patients with secondary progressive multiple sclerosis. Brain pathology (Zurich, Switzerland). 2004;14(2):164-74.

331. Howell OW, Reeves CA, Nicholas R, Carassiti D, Radotra B, Gentleman SM, et al. Meningeal inflammation is widespread and linked to cortical pathology in multiple sclerosis. Brain : a journal of neurology. 2011;134(Pt 9):2755-71. 
332. Stern JN, Yaari G, Vander Heiden JA, Church G, Donahue WF, Hintzen RQ, et al. B cells populating the multiple sclerosis brain mature in the draining cervical lymph nodes. Science translational medicine. 2014;6(248):248ra107.

333. Kabat EA, Glusman M, Knaub V. Quantitative estimation of the albumin and gamma globulin in normal and pathologic cerebrospinal fluid by immunochemical methods. The American journal of medicine. 1948;4(5):653-62. 334. Obermeier B, Mentele R, Malotka J, Kellermann J, Kumpfel T, Wekerle H, et al. Matching of oligoclonal immunoglobulin transcriptomes and proteomes of cerebrospinal fluid in multiple sclerosis. Nature medicine. $2008 ; 14(6): 688-93$.

335. Johnson SA, Cambier JC. Ageing, autoimmunity and arthritis: senescence of the B cell compartment - implications for humoral immunity. Arthritis Res Ther. 2004;6(4):131-9.

336. Grolleau-Julius A, Ray D, Yung RL. The role of epigenetics in aging and autoimmunity. Clinical reviews in allergy \& immunology. 2010;39(1):42-50.

337. Watad A, Bragazzi NL, Adawi M, Amital H, Toubi E, Porat BS, et al. Autoimmunity in the Elderly: Insights from Basic Science and Clinics - A MiniReview. Gerontology. 2017;63(6):515-23.

338. Rubtsov AV, Marrack P, Rubtsova K. T-bet expressing B cells - Novel target for autoimmune therapies? Cell Immunol. 2017;321:35-9.

339. Wang S, Wang J, Kumar V, Karnell JL, Naiman B, Gross PS, et al. IL-21 drives expansion and plasma cell differentiation of autoreactive CD11c(hi)Tbet(+) B cells in SLE. Nature communications. 2018;9(1):1758.

340. Blauth K, Owens GP, Bennett JL. The Ins and Outs of B Cells in Multiple Sclerosis. Frontiers in immunology. 2015;6:565.

341. Fraussen J ea. B cells of multiple sclerosis patients induce autoreactive proinflammatory $T$ cell responses. Clin Immunol. 2016;173:124-32.

342. Martorana A, Balistreri CR, Bulati M, Buffa S, Azzarello DM, Camarda C, et al. Double negative (CD19+IgG+IgD-CD27-) B lymphocytes: a new insight from telomerase in healthy elderly, in centenarian offspring and in Alzheimer's disease patients. Immunology letters. 2014;162(1 Pt B):303-9.

343. Jacobi AM, Reiter K, Mackay M, Aranow C, Hiepe F, Radbruch A, et al. Activated memory $B$ cell subsets correlate with disease activity in systemic lupus erythematosus: delineation by expression of CD27, IgD, and CD95. Arthritis and rheumatism. 2008;58(6):1762-73.

344. Frisullo G, Nociti V, Iorio R, Patanella AK, Marti A, Cammarota G, et al. Increased expression of $\mathrm{T}$-bet in circulating $\mathrm{B}$ cells from a patient with multiple sclerosis and celiac disease. Human immunology. 2008;69(12):837-9.

345. Frasca D, Diaz A, Romero M, D'Eramo F, Blomberg BB. Aging effects on Tbet expression in human B cell subsets. Cellular immunology. 2017;321:68-73.

346. Du SW, Arkatkar T, Jacobs HM, Rawlings DJ, Jackson SW. Generation of functional murine $\operatorname{CD} 11 \mathrm{C}(+)$ age-associated $\mathrm{B}$ cells in the absence of B cell T-bet expression. European journal of immunology. 2019;49(1):170-8.

347. L Michel CG, E Peelen, M Charabati , M.-A Lécuyer , L Bourbonnière , $S$ Larouche , P Duquette , A Bar-Or , J Gommerman , A Prat. ALCAM regulates B cells migration across the barriers of the central nervous system. ECTRIMS Online Library Michel L Sep 15, 2016; 146278. 2016.

348. Ms. Camille Grasmuck1, Dr. Laure Michel, MD PhD3, Dr. Evelyn Peelen, PhD4, Dr. Marc-Andre Lecuyer, PhD5, Dr. Stephanie EJ Zandee, PhD4, Dr. Tessa Dhaeze, PhD1, Dr. Jorge I Alvarez, PhD6, Mrs. Lyne Bourbonnière4, Ms. Sandra Larouche4, Dr. Pierre Duquette, MD, FRCPC7, Dr. Amit Bar-Or, MD6, Dr. Jennifer Gommerman, PhD8 and Dr. Alexandre Prat, MD, PhD, FRCPC9, . Activated 
Leukocyte Cell Adhesion Molecule Regulates B Cell Migration across Central Nervous System Barriers. Abstract Actrims 2019. 2019.

349. Bulati M, Buffa S, Martorana A, Candore G, Lio D, Caruso C, et al. Trafficking phenotype and production of granzyme $B$ by double negative $B$ cells $(\operatorname{IgG}(+) \operatorname{IgD}(-) \mathrm{CD} 27(-))$ in the elderly. Experimental gerontology. 2014;54:123-9. 350. Kunkel EJ, Butcher EC. Plasma-cell homing. Nature reviews Immunology. 2003;3(10):822-9.

351. Cagigi A, Mowafi F, Phuong Dang LV, Tenner-Racz K, Atlas A, Grutzmeier $\mathrm{S}$, et al. Altered expression of the receptor-ligand pair CXCR5/CXCL13 in B cells during chronic HIV-1 infection. Blood. 2008;112(12):4401-10.

352. Centuori SM, Gomes CJ, Kim SS, Putnam CW, Larsen BT, Garland LL, et al. Double-negative (CD27(-)IgD(-)) B cells are expanded in NSCLC and inversely correlate with affinity-matured $\mathrm{B}$ cell populations. Journal of translational medicine. $2018 ; 16(1): 30$.

353. Palma P, Rinaldi S, Cotugno N, Santilli V, Pahwa S, Rossi P, et al. Premature $B$-cell senescence as a consequence of chronic immune activation. Human vaccines \& immunotherapeutics. 2014;10(7):2083-8.

354. Buffa S, Bulati M, Pellicano M, Dunn-Walters DK, Wu YC, Candore G, et al. $B$ cell immunosenescence: different features of naive and memory $B$ cells in elderly. Biogerontology. 2011;12(5):473-83.

355. Klotz L, Havla J, Schwab N, Hohlfeld R, Barnett M, Reddel S, et al. Risks and risk management in modern multiple sclerosis immunotherapeutic treatment. Therapeutic advances in neurological disorders. 2019;12:1756286419836571.

356. Gajofatto A, Benedetti MD. Treatment strategies for multiple sclerosis: When to start, when to change, when to stop? World journal of clinical cases. 2015;3(7):545-55.

357. Aungst A, Casady L, Dixon C, Maldonado J, Moreo N, Pearsall L, et al. Assessing Barriers to Adherence with the Use of Dimethyl Fumarate in Multiple Sclerosis. Clinical drug investigation. 2019.

358. Berger JR. Classifying PML risk with disease modifying therapies. Multiple sclerosis and related disorders. 2017;12:59-63.

359. Diebold M, Altersberger V, Decard BF, Kappos L, Derfuss T, Lorscheider J. A case of progressive multifocal leukoencephalopathy under dimethyl fumarate treatment without severe lymphopenia or immunosenescence. Multiple sclerosis (Houndmills, Basingstoke, England). 2019;25(12):1682-5.

360. Motte J, Kneiphof J, Strassburger-Krogias K, Klasing A, Adams O, Haghikia $A$, et al. Detection of JC virus archetype in cerebrospinal fluid in a MS patient with dimethylfumarate treatment without lymphopenia or signs of PML. J Neurol. 2018;265(8):1880-2.

361. Fox RJ, Chan A, Gold R, Phillips JT, Selmaj K, Chang I, et al. Characterizing absolute lymphocyte count profiles in dimethyl fumarate-treated patients with MS: Patient management considerations. Neurology Clinical practice. 2016;6(3):2209.

362. Sainz de la Maza S, Medina S, Villarrubia N, Costa-Frossard L, Monreal E, Tejeda-Velarde A, et al. Factors associated with dimethyl fumarate-induced lymphopenia. Journal of the neurological sciences. 2019;398:4-8.

363. Hoxtermann S, Nuchel C, Altmeyer P. Fumaric acid esters suppress peripheral CD4- and CD8-positive lymphocytes in psoriasis. Dermatology (Basel, Switzerland). 1998;196(2):223-30.

364. Fleischer V, Friedrich M, Rezk A, Buhler $U$, Witsch $E$, Uphaus $T$, et al. Treatment response to dimethyl fumarate is characterized by disproportionate 
CD8+ $\mathrm{T}$ cell reduction in MS. Multiple sclerosis (Houndmills, Basingstoke, England). 2018;24(5):632-41.

365. Ciolac D, Luessi F, Gonzalez-Escamilla G, Koirala N, Riedel C, Fleischer V, et al. Selective Brain Network and Cellular Responses Upon Dimethyl Fumarate Immunomodulation in Multiple Sclerosis. Frontiers in immunology. 2019;10:1779. 366. Mehta D, Miller C, Arnold DL, Bame E, Bar-Or A, Gold R, et al. Effect of dimethyl fumarate on lymphocytes in RRMS: Implications for clinical practice. Neurology. 2019;92(15):e1724-e38.

367. Holm Hansen R, Hojsgaard Chow H, Christensen JR, Sellebjerg F, von Essen MR. Dimethyl fumarate therapy reduces memory $T$ cells and the CNS migration potential in patients with multiple sclerosis. Multiple sclerosis and related disorders. 2019;37:101451.

368. Longbrake EE, Cantoni C, Chahin S, Cignarella F, Cross AH, Piccio L. Dimethyl fumarate induces changes in B- and T-lymphocyte function independent of the effects on absolute lymphocyte count. Multiple sclerosis (Houndmills, Basingstoke, England). 2018;24(6):728-38.

369. Gross CC, Schulte-Mecklenbeck A, Runzi A, Kuhlmann T, Posevitz-Fejfar A, Schwab N, et al. Impaired NK-mediated regulation of T-cell activity in multiple sclerosis is reconstituted by IL-2 receptor modulation. Proceedings of the National Academy of Sciences of the United States of America. 2016;113(21):E2973-82.

370. Jiang W, Chai NR, Maric D, Bielekova B. Unexpected role for granzyme K in CD56bright NK cell-mediated immunoregulation of multiple sclerosis. Journal of immunology (Baltimore, Md : 1950). 2011;187(2):781-90.

371. Smith MD, Calabresi PA, Bhargava P. Dimethyl fumarate treatment alters NK cell function in multiple sclerosis. European journal of immunology. 2018;48(2):380-3.

372. Zaro BW, Vinogradova EV, Lazar DC, Blewett MM, Suciu RM, Takaya J, et al. Dimethyl Fumarate Disrupts Human Innate Immune Signaling by Targeting the IRAK4-MyD88 Complex. Journal of immunology (Baltimore, Md : 1950). 2019;202(9):2737-46.

373. Das RK, Brar SK, Verma M. Recent advances in the biomedical applications of fumaric acid and its ester derivatives: The multifaceted alternative therapeutics. Pharmacological reports : PR. 2016;68(2):404-14.

374. Piroli GG, Manuel AM, Patel T, Walla MD, Shi L, Lanci SA, et al. Identification of Novel Protein Targets of Dimethyl Fumarate Modification in Neurons and Astrocytes Reveals Actions Independent of Nrf2 Stabilization. Molecular \& cellular proteomics : MCP. 2019;18(3):504-19.

375. Traub J, Traffehn S, Ochs J, Hausser-Kinzel S, Stephan S, Scannevin R, et al. Dimethyl fumarate impairs differentiated B cells and fosters central nervous system integrity in treatment of multiple sclerosis. Brain pathology (Zurich, Switzerland). 2019.

376. Giovannoni G. Disease-modifying treatments for early and advanced multiple sclerosis: a new treatment paradigm. Current opinion in neurology. 2018;31(3):233-43.

377. Boyko AN, Boyko OV. Cladribine tablets' potential role as a key example of selective immune reconstitution therapy in multiple sclerosis. Degenerative neurological and neuromuscular disease. 2018;8:35-44.

378. Rommer PS, Milo R, Han MH, Satyanarayan S, Sellner J, Hauer L, et al. Immunological Aspects of Approved MS Therapeutics. Frontiers in immunology. 2019;10:1564. 
379. Braune S, Grimm S, van Hovell P, Freudensprung U, Pellegrini F, Hyde R, et al. Comparative effectiveness of delayed-release dimethyl fumarate versus interferon, glatiramer acetate, teriflunomide, or fingolimod: results from the German NeuroTransData registry. J Neurol. 2018;265(12):2980-92.

380. Rubtsov AV, Rubtsova K, Fischer A, Meehan RT, Gillis JZ, Kappler JW, et al. Toll-like receptor 7 (TLR7)-driven accumulation of a novel CD11C(+) B-cell population is important for the development of autoimmunity. Blood. 2011;118(5):1305-15.

381. Jenks SA, Cashman KS, Zumaquero E, Marigorta UM, Patel AV, Wang X, et al. Distinct Effector B Cells Induced by Unregulated Toll-like Receptor 7 Contribute to Pathogenic Responses in Systemic Lupus Erythematosus. Immunity. 2018;49(4):725-39.e6.

382. Martin V, Wu YC, Kipling D, Dunn-Walters DK. Age-related aspects of human IgM(+) B cell heterogeneity. Ann N Y Acad Sci. 2015;1362:153-63.

383. Fraussen J, Marquez S, Takata K, Beckers L, Montes Diaz G, Zografou C, et al. Phenotypic and Ig Repertoire Analyses Indicate a Common Origin of IgD()CD27(-) Double Negative B Cells in Healthy Individuals and Multiple Sclerosis Patients. Journal of immunology (Baltimore, Md : 1950). 2019;203(6):1650-64.

384. Dunn-Walters DK, Ademokun AA. B cell repertoire and ageing. Current opinion in immunology. 2010;22(4):514-20.

385. Franceschi C, Capri M, Monti D, Giunta S, Olivieri F, Sevini F, et al. Inflammaging and anti-inflammaging: a systemic perspective on aging and longevity emerged from studies in humans. Mechanisms of ageing and development. 2007;128(1):92-105.

386. Zhang W, Zhang $\mathrm{H}$, Liu S, Xia F, Kang Z, Zhang $Y$, et al. Excessive CD11C(+)Tbet $(+)$ B cells promote aberrant TFH differentiation and affinity-based germinal center selection in lupus. Proceedings of the National Academy of Sciences of the United States of America. 2019;116(37):18550-60.

387. Ma S, Wang C, Mao X, Hao Y. B Cell Dysfunction Associated With Aging and Autoimmune Diseases. Frontiers in immunology. 2019;10:318.

388. Knox JJ, Buggert M, Kardava L, Seaton KE, Eller MA, Canaday DH, et al. T-bet+ B cells are induced by human viral infections and dominate the HIV gp140 response. JCI insight. 2017;2(8).

389. Hritzo Ahye MK, Golding A. Cytoplasmic FOXO1 identifies a novel diseaseactivity associated $B$ cell phenotype in SLE. Lupus science \& medicine. 2018;5(1):e000296.

390. van Langelaar J, Rijvers $L$, Janssen $M$, Wierenga-Wolf $A F$, Melief $M J$, Siepman TA, et al. Induction of brain-infiltrating T-bet-expressing $B$ cells in multiple sclerosis. Annals of neurology. 2019;86(2):264-78.

391. Eggers EL, Michel BA, Wu H, Wang SZ, Bevan CJ, Abounasr A, et al. Clonal relationships of CSF $B$ cells in treatment-naive multiple sclerosis patients. JCI insight. 2017;2(22).

392. Buijs S, Krol M, de Voer G. Healthcare utilization and costs of multiple sclerosis patients in the Netherlands: a healthcare claims database study. Journal of comparative effectiveness research. 2018;7(5):453-62.

393. Sindic PC. Multiple sclerosis 2018 [Available from: https://www.fondationcharcot.org/nl/multiple-sclerose-charcot-stichting.

394. Dubois B, Kobelt G, Berg J, Capsa D, Gannedahl M. New insights into the burden and costs of multiple sclerosis in Europe: Results for Belgium. Multiple sclerosis (Houndmills, Basingstoke, England). 2017;23(2_suppl):29-40. 
395. Greenfield AL, Hauser SL. B-cell Therapy for Multiple Sclerosis: Entering an era. Annals of neurology. 2018;83(1):13-26.

396. Phase II Study of Interleukin-21 (rIL-21) vs Dacarbazine (DTIC) in Patients With Metastatic or Recurrent Melanoma [Internet]. Available from: https://clinicaltrials.gov/ct2/show/NCT01152788.

397. Schmidt H, Brown J, Mouritzen U, Selby P, Fode K, Svane IM, et al. Safety and clinical effect of subcutaneous human interleukin-21 in patients with metastatic melanoma or renal cell carcinoma: a phase I trial. Clinical cancer research : an official journal of the American Association for Cancer Research. 2010;16(21):5312-9.

398. Anwar MA, Shah M, Kim J, Choi S. Recent clinical trends in Toll-like receptor targeting therapeutics. Medicinal research reviews. 2019;39(3):105390. 


\section{Curriculum Vitae}

Gwendoline Montes Diaz werd geboren op 23 mei 1991 te Hasselt. In 2009 behaalde ze haar diploma Algemeen Secundair Onderwijs (ASO) in de afstudeerrichting Moderne talen - Wetenschappen aan het Virga Jessecollege te Hasselt. Vervolgens startte ze haar opleiding Biomedische Wetenschappen aan de Universiteit Hasselt / transnationale Universiteit Limburg waar ze in 2012 haar diploma bachelor in de Biomedische Wetenschappen behaalde. Aansluitend voltooide ze de master in de Biomedische Wetenschappen eveneens aan de Universiteit Hasselt binnen de afstudeerrichting 'clinical molecular sciences' waar ze in 2014 afstudeerde met grote onderscheiding samen met het certificaat proefdierkunde. Haar senior stage voerde ze uit in het Biomedisch Onderzoeksinstituut van de Universiteit Hasselt in het labo van prof. dr. Jerome Hendriks en was getiteld "Plant sterols as therapeutic approach for remyelination in multiple sclerosis". Hierin werd ze begeleid door dr. Tim Vanmierlo en Jo Mailleux. Daaropvolgend startte ze een joint doctoraatsopleiding in de Biomedische Wetenschappen aan de Universiteit Hasselt en Universiteit Maastricht (Bidiplomering) onder het promoterschap van prof. dr. Veerle Somers en prof. dr. Raymond Hupperts. Gedurende haar doctoraat focuste ze zich op de effecten van DMF op het immuunsysteem van multiple sclerosis patiënten en de rol van DN $B$ cellen in multiple sclerosis en schreef ze en behaalde ze een BOF beurs en een beurs van de MS-liga Vlaanderen. Daarnaast nam ze deel aan verschillende ondersteunende cursussen vanuit de Doctoral School of Medicine and Life Sciences en participeerde ze actief mee in het onderwijs aan de universiteit Hasselt. Tot slot nam ze deel aan verschillende congressen en won ze de posterprijs op de EMBO workshop To-B or not to-B: B-cells in health and disease (Gerona, Spanje). 


\section{Bibliography}

\section{Publications}

Dimethyl fumarate induces a persistent change in the composition of the innate and adaptive immune system in multiple sclerosis patients

Montes Diaz G., Fraussen J., Van Wijmeersch B., Hupperts R. and Somers V. Scientific reports 2018 may; 8(1):8194 (IF: 4.122)

Dimethyl fumarate treatment in multiple sclerosis: recent advances in clinical and immunological studies

Montes Diaz G., Hupperts R., Fraussen J.*, Somers V.*

* Equally contributing authors

Autoimmunity reviews, 2018 Dec;17(12):1240-1250. (IF: 8.745)

Phenotypic and Ig Repertoire Analyses Indicate a Common Origin of IgD(-)CD27() Double Negative B Cells in Healthy Individuals and Multiple Sclerosis Patients. Fraussen J, Marquez S, Takata K, Beckers L, Montes Diaz G, Zografou C, Van Wijmeersch B, Villar LM, O'Connor KC, Kleinstein SH, Somers V J Immunol. 2019 Sep15;203(6):1650-1664. (IF: 4.718)

\section{Oral presentations}

Immune regulation by dimethyl fumarate (DMF) in relapsing-remitting multiple sclerosis patients

Montes Diaz G., Fraussen J., Van Wijmeersch B., Hupperts R. and Somers V. 7e Wetenschappelijk Symposium Zuyderland, June $23^{\text {th }}$ 2016, Herent, The Netherlands

Dimethyl fumarate reduces the frequency and function of inflammatory immune cells in relapsing-remitting multiple sclerosis patients

Montes Diaz G., Fraussen J., Van Wijmeersch B., Hupperts R. and Somers V.

- WOG-MS symposium, October 24th 2017, Hasselt, Belgium

- MS research days, November 17 - 18 ${ }^{\text {th }} 2017$, Tiel, The Netherlands 
Dimethyl fumarate induces a persistent change of the innate and adaptive immune system of relapsing-remitting multiple sclerosis patients

Montes Diaz G., Fraussen J., Van Wijmeersch B., Hupperts R. and Somers V. 11the international congress on Autoimmunity, Mai 16 - 20 th 2018, Lisbon, Portugal

\section{Poster presentations}

Immune regulation by dimethyl fumarate (DMF) in relapsing-remitting multiple sclerosis patients

Montes Diaz G., Fraussen J., Van Wijmeersch B., Hupperts R. and Somers V.

- 6e Wetenschappelijk Symposium Atrium-Orbis, June 25th 2015 Sittard, The Netherlands

- MS research days, November 17 - 18 ${ }^{\text {th }}, 2016$ Amsterdam, The Netherlands

Dimethyl fumarate reduces the frequency and function of proinflammatory B cells while increasing anti-inflammatory $B$ cells in multiple sclerosis patients Montes Diaz G., Fraussen J., Van Wijmeersch B., Hupperts R. and Somers V. EMBO workshop To-B or not to-B: B-cells in health and disease, September 10 $13^{\text {th }} 2017$ Gerona, Spain

Dimethyl fumarate reduces the frequency and function of inflammatory immune cells in relapsing-remitting multiple sclerosis patients

Montes Diaz G., Fraussen J., Van Wijmeersch B., Hupperts R. and Somers V. MSParis2017 - $7^{\text {th }}$ joint ECTRIMS-ACTRIMS Meeting, October 25 - 28 $8^{\text {th }} 2017$, Paris, France

\section{Awards and grants}

EMBO Travel grant

Best poster presentation

EMBO workshop To-B or not to-B: B-cells in health and disease 2017, Gerona, Spain

Mobility grant Doctoral Schools of Hasselt University

Research stay Stockholm, Sweden January 2017 
BOF Grant 2015

Immune regulation by dimethylfumarate (DMF) in patients with relapsingremitting multiple sclerosis.

MS-liga Vlaanderen 2018

Antibody-dependent and -independent B cell functions in progressive multiple sclerosis 


\section{Dankwoord}

We zijn aan het einde gekomen van dit verhaal en dus is het hoog tijd om heel wat mensen te bedanken die ervoor gezorgd hebben dat ik zover ben geraakt. Ik heb vier jaar met heel veel plezier mijn doctoraat uitgevoerd en dit is mede te danken aan al mijn toffe collega's.

Als eerste een woord van dank aan mijn promotor Veerle. Ik wil je bedanken om aan mij te denken wanneer je een kandidaat zocht voor dit joint-PhD-project. Ik was er onmiddellijk enthousiast over en je hebt mij gedurende deze vier jaren op de voet gevolgd. Je zorgde ervoor dat ik tot het uiterste ging en je was altijd heel erg betrokken. Mijn chaotisch creatieve kant en jouw perfectionisme botsten wel af en toe, maar hierdoor heb ik heel veel van jou geleerd en was het eindresultaat altijd een pareltje - door ons teamwork. Ik wil je ook bedanken voor al de kansen die ik van jou heb gekregen. Ik heb veel ervaring opgedaan en een persoonlijke groei mogen doormaken, bijvoorbeeld ook door een week alleen naar een B-cel congres in Spanje te gaan en door een week in Zweden te mogen werken in het labo van prof. dr. Nilsson. Samen op congres gaan was ook altijd heel gezellig en hier hebben we ook heel veel plezier beleeft! Ik zal onze leuke trip naar Lissabon niet snel vergeten en ben blij dat ik je over die brug heb kunnen helpen $(-)$ en we een gezellig feestje hadden met prof. dr. Shoenfeld. Kortom ik wil je bedanken voor al je steun, al je kostbare tijd en al de toffe momenten!

Raymond, mijn tweede promotor, bedankt voor al je hulp vanaf het prille begin. Je zorgde ervoor dat ik me thuis voelde en goed werd opgevangen door je fantastische team van MS-verpleegkundigen in Zuyderland. Ik wil je bedanken voor je enorme hulp bij het verzamelen van de vele bloedstalen. Ik werd altijd heel blij van je enthousiaste e-mails en je enthousiasme over mijn werk tijdens de vergaderingen. Dit werkte altijd zeer motiverend. Ik wil je ook bedanken voor het delen van je klinische inzichten en het snel en grondig nalezen van mijn artikels en de thesis.

Mijn co-promoter Judith, bedankt om me in het labo op weg te helpen met de FACS en voor het delen van je uitgebreide kennis over B-cellen. Ik heb enorm veel van jou geleerd. Ik kon op elk moment van de dag bij je binnenlopen als ik ergens op vastliep of met de vraag of je even naar de B-cellen wou komen kijken 
en je maakte altijd tijd voor me vrij. Wanneer het wat moeilijker was stelde je me altijd gerust dat experimenten nu eenmaal mislukken, maar dat alles wel goedkomt! Ik heb ook genoten van onze vele gesprekken op je bureau wanneer we het over je schattige zoontjes hadden. Ik wil je ook bedanken voor het vele naleeswerk en verbeteringen gedurende mijn hele doctoraat! Bedankt dat ik altijd op je kon rekenen!

I would like to thank the members of the Jury, Prof. dr. Peter Feys, Prof. dr. Niels Hellings, dr. Luisa Villar, Prof. dr. Sandra Amor for critically reviewing my thesis and for your good advice and improvements. Prof. dr. Marc de Baets for being the chairman of my jury and helping me during the end phase of my PhD and dr. Jan Damoiseaux and Prof. dr. Bert Op 't Eijnde for being members of my jury.

Niels, ik wil jou bedanken omdat ik bij jou en je team altijd terecht kon om beroep te doen op jullie expertise rond de T-cellen. Het was altijd gezellig om samen met de T-cel groep op congres te gaan. Een congres met jou erbij moest afgesloten worden ofwel met cocktails, ofwel met een feestje of salsadansen $(-)$ wat zorgde voor veel sfeer en gezelligheid op Biomed. Ik wil je ook bedanken voor je waardevolle input en het grondig nalezen van mijn artikel en deze thesis!

Vervolgens wil ik graag alle mensen bedanken die ervoor gezorgd hebben dat ik zo een mooie collectie aan stalen heb mogen verzamelen. Hiervoor bedank ik alle MS-patiënten en gezonde controles die deelgenomen hebben aan deze studie en hun tijd en bloed gedoneerd hebben. Daarnaast wil ik ook Prof. Dr. Bart Van Wijmeersch, prof. dr. Veronica Popescu en de werknemers van het Revalidatie en MS centrum Overpelt voor het helpen bij het verzamelen van de bloedstalen. Ik wil ook heel graag Prof. dr. Hupperts nogmaals bedanken, alsook zijn geweldig team van MS-verpleegkundigen, namelijk Sandra Liedekerken, Tiny Kempkens, Judith Poeth, Ingrid Mevissen, Bertine Timmermans, Mariëlle Moreau-Geertjens, Jolanda Biermans, Maartje Verhagen en Lianne Denessen voor het verzamelen van bloedstalen en klinische gegevens. Sandra, ik vond het heel leuk dat je de toegewezen persoon was voor mijn project en ik dus altijd kon rekenen op je expertise, je hulp bij de ethische commissie en de documentatie van de klinische gegevens in de binders. Ik voelde me altijd zeer welkom in Zuyderland. Daarnaast wil ik ook graag de Biobank bedanken voor het 
verwerken, opslaan en documenteren van al de patiënten stalen. Kim Ulenaers, samen hebben we uren langs elkaar gespendeerd tijdens het verwerken van de bloedstalen, bedankt voor de leuke babbels en al je werk voor de Biobank. Ik wil ook heel graag Véronique en Igna bedanken om altijd klaar te staan om tijdens hun drukke werkdag bloed te prikken bij gezonde controles. Zonder jullie allemaal was er geen bloed en dus geen onderzoek, dus heel hard bedankt hiervoor!

Ik wil ook heel graag Biogen Nederland bedanken voor de financiële ondersteuning en meer bepaald Rob van den Brule en Thijs Koster voor hun vertrouwen, enthousiasme en de opvolging van het project. Ik wil ook graag de Universiteit Hasselt en de Universiteit van Maastricht bedanken voor de financiële ondersteuning van mijn project.

Het hele team van labotechniekers namelijk Igna, Katrien, Christel, Leen en Kim zou ik ook heel graag even willen bedanken. Jullie zorgen voor zoveel orde en netheid in de labo's en hebben zoveel labo ervaring wat jullie ook met alle plezier met alle doctoraatstudenten delen. Jullie houden de hele boel draaiende en zorgen voor een zeer aangename werkomgeving. Daarnaast was het ook altijd gezellig om samen te lunchen en heb ik veel levenslessen van jullie mogen leren. Naast de labotechniekers wil ik ook graag de secretariaat medewerkers, namelijk Véronique, Rani en Christina, bedanken. Ik kon altijd bij jullie binnenlopen voor een praatje en als ik een administratief probleem had losten jullie dit dadelijk op. Dankjewel voor alle hulp en ook voor Véronique voor het helpen met al de administratie en geregel van de eindfase van mijn doctoraat.

Dan is het tijd om mijn verschillende bureaugenootjes te bedanken, waarmee ik toch wel het meeste van mijn tijd heb gespendeerd. Als jong kuiken in de hoop mocht ik de bureau delen met de meer ancien doctoraatstudenten namelijk Nele, Stelios en Ilse. Nele, jij hebt me laten zien hoe stresserend het laatste jaar kan zijn en hebt mij hier goed op voorbereid. Het was ook leuk om zo vroeg in mijn doctoraat jouw verdediging te kunnen meemaken. Stelios, I have to admit that I totally misjudged you. You looked a little angry and I was actually a little afraid of you at the beginning. However, after getting to know you and tearing down the wall bit by bit I got to know the sweetest Greek man ever! Thank you for staying in Biomed (and watching football) when I had to work until 12.00 at night so that I would not be alone in the building and would not have to set the alarm. This 
made me feel safe so I could concentrate on my experiments. I even once locked myself out of Biomed by forgetting my key in the building and I only had to call you and you drove immediately back from home to help me! I really appreciate that! We had some good times! Ilse, met jou heb ik het langste de bureau gedeeld en je bent hierdoor ook een goede vriendin geworden! Je zorgde voor een heel toffe sfeer in de bureau waar veel gelachen moest worden, waar kerstliedjes moesten gezongen worden, waar gedanst moest worden, waar orchideeën wonderbaarlijk mooi groeiden door jouw groene vingers (en stierven na je vertrek door mijn niet zo groene vingers...), waar bureauactiviteiten werden georganiseerd, waar ik uiteindelijk je bubbel heb mogen verkleinen, waar kebabkes werden gegeten, waar lief en leed werd gedeeld en waar vooral veel relativeringsvermogen werd opgedwongen voor mijn eigen goed. Bedankt om mij te steunen wanneer mijn experimenten weer eens niet lukten en samen met mij te vieren wanneer ze wel lukten en er andere succesverhalen waren! Zonder jou was het een saaie boel geweest! Je bent een topmadam! Dana, na de grote verhuis kwam je bij ons in de bureau terecht. We werden beide goed opgevangen door Ilse en hebben met ons drietjes toch wel heel veel uren gebabbeld over onze experimenten, schrijven, elkaars werk nagelezen, elkaar proberen te helpen waar mogelijk, en vaak ook gewoon een klankbord zijn voor elkaar zijn! Bedankt voor de leuke tijd samen! Rut, you were the last one to join our desk. My first impression of you was wrong, but every good friendship started with "When I first met you I thought you were a b*tch". Over the years you became one of my dearest friends! We could talk for hours and some days we really had to go into the lab to stop the talking and do some work. We are kindred spirits and I love that we can be real with each other (while others perceive it is as mean (-) it is just the Spanish way to express ourselves!). I loved having you as a colleague and I still miss our time in the desk, even your grumpy morning face (-)! Thanks for bringing joy in the desk during my last year and I hope I have helped you put things in perspective a bit for your PhD just like Ilse did with me. I know you didn't always like that I was mothering you but you needed someone in your corner! It was amusing and a lovely distraction to witness the 'no, Maxim is only a friend' struggle changing into 'I have to tell you something...'. I'm glad you are in safe hands now, so that I can lose some grip on the mothering (?). You are an amazing 
woman, a brilliant scientist with a bright future ahead and you will be a fantastic godmother to my little daughter.

Dan wil ik even de postdocs van mijn onderzoeksgroep bedanken, Patrick, Elke en Liesbeth bedankt voor de leuke babbels, de begeleiding, het advies en de toffe tijd! Gedurende mijn doctoraat heb ik vier jaar lang verschillende studenten begeleid die ik ook wil bedanken voor hun harde werk en waardoor ik ook mijn roeping heb gevonden om verder te gaan als leerkracht, aangezien ik dit met heel veel plezier deed. Vooral Lien mag ik als student zeker niet vergeten! Lien je hebt bij mij je jaarwerkstuk geschreven, je junior stage gedaan en ook je senior stage gedaan. Je bent een enorme harde werker die tot de laatste uurtjes samen met mij in het labo bleef. Ook al stuurde ik je al uren ervoor naar huis je wou toch blijven tot het hele experiment gedaan was. Ik heb je kunnen zien groeien van een heel onzekere student tot een doctoraatstudent die nu wel wat steviger in haar schoenen durft te staan en daar ben ik heel blij om. Lien, blijf zo gemotiveerd en gepassioneerd verder je onderzoek doen en zoals ik altijd zei alles komt uiteindelijk wel goed. Je gaat een heel mooi doctoraat afleggen en dan kom ik zeker kijken! Je hebt het laatste jaar van mijn doctoraat nog plezanter gemaakt en ik vond het heel tof om samen met jou te werken!

Dan mijn Biomedcollega's, eerst en vooral de macrofaaggroep: Jo, Elien W., Elien G., Suzan, Tim, Jeroen, Jerome en de T-cel groep: Marjan, Bieke, Cindy, Evelien, Liesbet, Pauline, bedankt voor de babbeltjes, de toffe congressen samen en de feestje die daarbij horen, en zeker ook de hulp met experimenten. In het bijzonder Bieke bedankt voor al de hulp met de migratie assay en om al je kennis te delen over de T-cellen.

Daarnaast ook alle andere biomedcollega's zoals Selien, Daniëlla, Annelies B., Petra, Leen, Melissa L.M. en al de rest die ik vergeten ben op te noemen, waar ik vaak een babbeltje mee maakten in de gangen, tijdens het labo werk, in de koffieruimte of tijdens lunch en die zorgen voor een super toffe sfeer op het biomedfeest en de BBQ.

Ook even een dankjewel aan al mijn vrienden die voor de nodige afleiding en ontspanning zorgden. Eline, Sarah, Glenda en Ilke onze al minstens 14 jaren vriendschap is van onschatbare waarde, dankjewel om al mijn gezaag en frustratie 
van mijn doctoraat aan te horen maar ook om samen met mij de successen te vieren. Dan mijn UH roeimeisjes Sarah en Elien, we hebben altijd een super tijd samen net zoals vroeger op het water! Elien jij kon altijd leuk meepraten over mijn Biomedavonturen omdat je daar ook iedereen kent en Sarah we zaten als doctoraatstudenten een beetje in hetzelfde scheutje. Dan mijn animatieploeg van Intersoc, Jurgen, Wendy, Thibaut, Olli, Martijn, Lieze en Monika de zomervakanties en skivakantie zorgde ervoor dat ik volledig kon ontspannen en er me volle goesting terug aan kon beginnen. En tot slot mijn huidige collega's en in het bijzonder Freya, Nele, Gisèle, Sarah, Jeffrey, Stijn, Johan, Jolien, Sofie en Ward om het mogelijk te maken om mijn eindfase van mijn doctoraat te combineren met mijn nieuwe job en om af en toe te met veel interesse te checken hoe alles verder verliep en in waar ik nu precies zat in de eindfase. Stijn bedankt voor je vriendschap en om al mijn gezaag aan te horen tijdens onze wekelijkse koffiedate en voor de toffe wijnavonden met culinaire hoogstandjes waardoor ik alles opnieuw kon relativeren. Maar ik wil ook zeker al de andere collega's bedanken voor de leuke sfeer op het werk! Ik wil ook even mijn leerlingen bedanken die zeer geïnteresseerd waren in de inhoud van mijn doctoraat en maar niet konden begrijpen dat ik ervoor kies om aan hun les te geven, ik verwacht hier zeker ook goede toekomstige wetenschappers tussen!

Dan mijn Familie, ik wil al mijn nonkels en tantes en nichten en neven en mijn schoonbroer Gino bedanken om er altijd te zijn. Vooral tante Nadine, mijn peter Ronny, mijn meter Marina en nonkel Dany waar ik altijd terecht kan en om altijd te vragen hoe het met mijn doctoraat ging en om geduldig te luisteren ook al was het moeilijk om er een beeld van te kunnen vormen. Maar ook mijn lieve Bomma die op het begin van mijn doctoraat nog thuis woonde en die ook iedere keer vroeg hoe het op 'school' was en me altijd geruststelde dat alles wel goed kwam, want ik kan alles wat ik wil volgens haar. Mijn schoonouders Danny en Brigitte en schoonzus en broer Leen en Bert wil ook even bedanken voor alle steun en interesse die ze lieten zien tijdens mijn doctoraat.

Dan wil ik mijn grote zus Angelina bedanken! Bedankt om altijd te geloven in je kleine zusje en om trots op mij te zijn. Het is leuk om een toffe zus te hebben die ik elke week (bijna elke dag) zie en waar ik alles mee kan delen! Alyssa, mijn metekindje, en Aryana jullie brengen ook heel veel vreugde in mijn leven. 
Mijn ouders, mama en papa, zonder jullie zou ik nu niet mijn dankwoord aan het schrijven zijn. Jullie hebben heel jullie leven meerdere jobs tegelijk uitgevoerd en heel hard gewerkt zodat ik een beter leven zou kunnen hebben, waar ik zoals jullie altijd zeggen hard met mijn hoofd zal werken en niet zoals jullie harde fysieke arbeid zal moeten verrichten. Zonder al jullie harde werk had ik in de eerste plaats al niet kunnen verder studeren en daarbovenop hebben jullie toch ook altijd gezorgd dat er heel veel quality time was samen met het gezin. Jullie zijn mijn grote voorbeeld en ik kan alleen maar hopen dat ik mijn kinderen even goed kan opvoeden als jullie. Jullie hebben altijd in mijn geloofd en mij gesteund en zeiden dat alles altijd wel goed komt. De eerste twee jaren van mijn doctoraat woonde ik nog thuis en stond er altijd eten klaar, zelfs als ik weer om middernacht thuiskwam, en kwamen jullie mij dan ook eerst nog halen als er geen bussen meer reden. Ik kan jullie niet hard genoeg bedanken voor alles wat jullie voor mij gedaan hebben!

Tot slot mijn gezin, te beginnen met mijn rots in de branding: mijne man Erwin. Je bent mijn grootste steun en toeverlaat en zonder jou was dit ook zeker niet gelukt. Bedankt om elke dag te luisteren naar hoe mijn dag was geweest, om zo lief te zijn om 's avonds wanneer ik weer eens onverwacht tot laat in het labo zat om eten te komen brengen zodat we toch samen konden eten en dan geduldig te wachten tot mijn experimenten klaar waren. Bedankt om mij overal te brengen en te komen halen, wanneer ik geen zin had om te rijden of in de tijd dat ik zwanger was van Lucas het vaak niet durfde. Bedankt voor al de uren die je gestoken hebt in het nalezen van mijn beurzen, ethische commissies, papers en stukjes van deze thesis zodat er geen taalfout meer in zou staan. Ik ben ook heel blij dat je me ten huwelijk hebt gevraagd tijdens mijn doctoraat en het was zo lief dat je heel veel van de planning ervan en de e-mails erover op je nam zodat ik me kon focussen op mijn doctoraat en ik enkel nog moest kiezen tussen de drie laatste opties van alles. Ik wil je ook vooral bedanken voor al je geduld; mijn doctoraat kwam vaak genoodzaakt op de eerste plaats wanneer ik lastminute bijvoorbeeld nog iets moest afwerken in het weekend en we een deel van de optredens van Pinkpop misten en ik in het vliegtuig naar Miami nog de laatste aanpassingen aan mijn paper moest schrijven en ook nog in de avonden van de eerste week van onze roadtrip doorheen Amerika. Dit was niet leuk voor jou maar je liet me eraan werken, omdat je wist dat dit belangrijk voor mij was. Zelfs tijdens 
de ziekenhuisopname bij de eerste weeën van Lucas liet je mij mijn laatste onderzoek hoofdstuk tot 1uur 's nachts verbeteren omdat je wist dat ik dit af wou hebben voor onze kleine schat geboren was. Ik weet dat ik heel veel van je hebt gevraagd gedurende deze periode en ik kan mijn geen betere man inbeelden die zonder zeuren en met zoveel liefde er altijd voor mij is. Weet dat ik jou en alles wat je voor me doet enorm apprecieer. Dankjewel allerliefste schat! Nu zullen we als gezinnetje terug wat meer quality time hebben en schrijven we verder aan ons verhaal.

Dan als laatste mag ons zoontje Lucas hier zeker niet in ontbreken. Dit boekje ben ik beginnen schrijven wanneer ik zwanger was van jou, wat ervoor zorgde dat ik alles wat kon relativeren aangezien ik niet meer te veel mocht stressen, want jouw gezondheid kwam nu op de eerste plaats. Liefste schat, je brengt zoveel liefde in mijn leven en het was heel moeilijk om mezelf te verplichten om iedere keer weer te werken aan de afronding van dit boekje terwijl ik natuurlijk het liefste bij jou wil zijn. Ik ben blij en opgelucht dat we nu aan het einde zijn gekomen en dat ik mijn vrije tijd nu terug volledig met jou kan spenderen, en binnenkort ook met je kleine zus Valentina. Mijn liefste schatten, ik hoop dat dit boekje een inspiratie voor jullie kan zijn dat jullie alles kunnen worden of doen wat je maar wil zolang jullie hartje het maar genoeg wilt en jullie bereid zijn om het vol te houden tot het einde. Ik draag dit boekje dus ook heel graag op aan jullie.

Gwendoline, augustus 2020

'Being a scientist is like being an explorer. You have this immense curiosity, this stubbornness, this resolute will that you will go forward no matter what other people say' - Sara Seager 